\title{
Aufhebung der Benutzungspflicht von Radwegen
}

Thomas Richter

Stephan Ruhl

Jörg Ortlepp

Marcel Schreiber

Unfallforschung der Versicherer 


\section{Aufhebung der Benutzungspflicht von Radwegen}

Univ.-Prof. Dr.-Ing. Thomas Richter

Dipl.-Ing. Stephan Ruhl

Dipl.-Ing. Jörg Ortlepp

Dipl.-Ing. Marcel Schreiber

Unfallforschung

der Versicherer 


\section{Impressum}

Gesamtverband der Deutschen Versicherungswirtschaft e. V.

Unfallforschung der Versicherer

Wilhelmstraße 43/43G, 10117 Berlin

Postfach 0802 64, 10002 Berlin

E-Mail: unfallforschung@gdv.de

Internet: www.udv.de

Facebook: www.facebook.com/unfallforschung

Twitter: @unfallforschung

YouTube: www.youtube.com/unfallforschung

ISBN-Nr.: 978-3-939163-82-4

Redaktion: Dipl.-Ing. Jörg Ortlepp, Dipl.-Ing. Marcel Schreiber

Bildnachweis: UDV und siehe Quellenangaben

Erschienen: 04/2018 


\title{
Aufhebung der Benutzungspflicht von Radwegen
}

\author{
Bearbeitet durch: \\ Technische Universität Berlin \\ Fachgebiet Straßenplanung und Straßenbetrieb \\ Univ.-Prof. Dr.-Ing. Thomas Richter \\ Dipl.-Ing. Stephan Ruhl

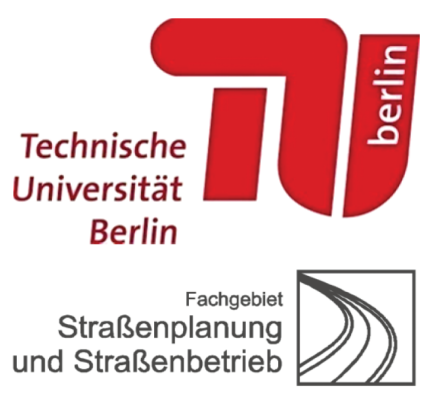

Bei der UDV betreut von:

Dipl.-Ing. Jörg Ortlepp Dipl.-Ing. Marcel Schreiber

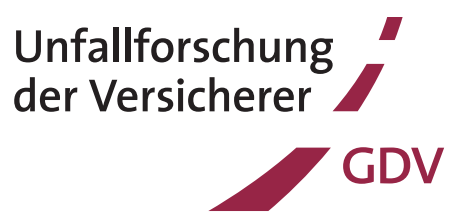





\section{Kurzfassung}

Ziel des Forschungsvorhabens war es, die Veränderungen des Unfall- und Konfliktgeschehens sowie des Verkehrsverhaltens an Radwegen mit Aufhebung deren Benutzungspflicht zu analysieren. Gemäß der Literaturanalyse sind Verkehrssicherheit und Fahrverhalten sowie verschiedenartige Begleitmaßnahmen mit Aufhebung der Radwegebenutzungspflicht noch nicht mit einem umfangreichen Streckenkollektiv untersucht worden.

Die eingangs durchgeführte Online-Befragung zeigt die erfahrungsbasierten Erkenntnisse und subjektiven Bewertungen zur Thematik aus 356 Städten. In Deutschland existieren demnach keine einheitlichen Vorgehensweisen im Umgang mit der Radwegebenutzungspflicht hinsichtlich Begleitmaßnahmen und Anordnungskriterien. Viele Kommunen berichten zudem von Akzeptanzproblemen bei Radfahrern und Autofahrern bezüglich des Radfahrens auf der Fahrbahn.

Die Unfallanalyse betrachtet 108 nicht benutzungspflichtige Radwege auf 84,1 Kilometern mit 2.243 Unfällen mit Personenschaden und davon 741 Radunfälle. In der Gesamtentwicklung zeigt sich mit Aufhebung der Radwegebenutzungspflicht eine Zunahme der Radunfälle. Die Unfallkenngrößenberechnung zeigt an den vordergründig untersuchten Radwegen mit Aufhebung der Radwegebenutzungspflicht ohne Begleitmaßnahmen unter Berücksichtigung der Radverkehrsentwicklung jedoch keine Veränderungen der Verkehrssicherheit. Die Unfallentwicklung ist somit mit der Radverkehrsentwicklung erklärbar. Die kombinierte makroskopische und mikroskopische Unfallanalyse zeigt für Radwege ohne weitere Begleitmaßnahmen mit Aufhebung der Benutzungspflicht aufgrund der vorwiegenden Radwegweiternutzung kaum strukturelle Veränderungen. Verlagerungen des Radunfallgeschehens auf die Fahrbahn bestätigen sich nicht.

Die Verkehrsverhaltensanalyse an 10 Radwegen mit und 10 Radwegen ohne Benutzungspflicht bestätigt die seltene Fahrbahnnutzung bei keinen oder nur geringfügigen Begleitmaßnahmen (Piktogrammen) mit Aufhebung der Radwegebenutzungspflicht. Deutliche Verlagerungen des Radverkehrs auf die Fahrbahn zeigen sich nur bei Schutz- bzw. Radfahrstreifen bei gleichzeitigem Rückbau des Radweges. In der Verkehrskonfliktanalyse zeigen sich die Schwerpunkte im Konfliktgeschehen entsprechend der identifizierten Unfallschwerpunkte. Als besonders kritisch ist das Einund Abbiegen an Knotenpunkten anzusehen, dies unabhängig der Radwegebenutzungspflicht. Da keine Verhaltensänderungen nachweisbar sind, gibt es auch im Unfall- und Konfliktgeschehen keine wesentlichen Veränderungen.

Maßgebend hierfür ist der hohe Stellenwert der Verkehrssicherheit für die Wahl der Flächennutzung der Radfahrer und das hohe subjektive Sicherheitsempfinden beim Radwegfahren, wie die abschließenden Vor-Ort-Befragungen der Verkehrsteilnehmer zeigen. Die verkehrsrechtlichen Regelungen zur Radwegebenutzungspflicht sind den Verkehrsteilnehmern zudem größtenteils unbekannt und für das Verkehrsverhalten nicht entscheidend.

Die vorliegende Untersuchung zeigt im Gesamtergebnis, dass sich mit Aufhebung der Radwegebenutzungspflicht bei keinen oder nur geringfügigen Begleitmaßnahmen (Piktogrammen) keine Veränderungen in der Flächennutzung und entsprechend auch nicht im Unfall- und Konfliktgeschehen einstellen. Änderungen zeigen sich nur, wenn Angebote für das Fahrbahnfahren geschaffen werden (Schutz- oder Radfahrstreifen inkl. Radwegrückbau). Die Benutzungspflicht allein hat dagegen keinen Einfluss auf das Fahrverhalten und die Verkehrssicherheit an Radwegen. Grundlegend müssen Radwege unabhängig deren Benutzungspflicht einen ausreichenden Standard aufweisen. Im Rahmen der Verkehrssicherheitsarbeit müssen weiterhin die bereits bekannten Unfall- und Konfliktschwerpunkte entschärft werden. Nur so kann die Verkehrsplanung den Anforderungen und Sicherheitserfordernissen des steigenden Radverkehrsaufkommens gerecht werden. 


\section{Abstract}

The main goal of this research was the evaluation of changes in accident and conflict occurrence as well as cycling behaviour at cycle paths with the suspension of the obligation of their usage (change from mandatory to advisory cycle paths). The literature shows, that road safety, cycling behaviour and accompanying measures when suspending the obligation of cycle path usage are not yet analysed with a comprehensive collective of research objects.

The initial online-survey illustrates the experiences and subjective ratings on this topic for 356 German cities. The results highlight, that consistent approaches dealing with the obligation of cycle path usage regarding accompanying measures and criteria for general regulation do not exist in Germany. Regarding cycling on the carriageway many cities reported problems of acceptance for cyclists and car drivers.

The accident analysis of this project considers 108 advisory cycle paths in the extent of 84,1 kilometers with 2.243 accidents with personal damage, thereof 741 cycle accidents. Generally, the overall trend shows an increase of cycle accidents with the suspension of the obligation of cycle path usage. In contrast, the calculated accident parameters taking account of the increase of cycle traffic indicate no changes in road safety for the largest collective of advisory cycle paths without any accompanying measures. Therefore, the increase of cycle accidents is explainable with the general increase of cycle traffic. The combined macroscopic and microscopic accident analysis shows for advisory cycle paths without any accompanying measures just marginally changes when the obligation of their usage was suspended. This is the result of the predominant further usage of the cycle paths. Shifting's of cycle accidents to the carriageway cannot be confirmed.

The additional analysis at 10 mandatory cycle paths and 10 advisory cycle paths confirms also the rare usage of the carriageway, when no or just simple accompanying measures (pictograms) are implemented with the suspension of the obligation of cycle path usage. Relevant shifting's of cyclists emerge just with the marking of cycle lanes or advisory lanes on the carriageway including the removal of the former cycle path. The conflict analysis highlight the main conflicts correlating with the already identified main types of accidents. Particularly turn off and turn into accidents at road junctions are the most critical cases. Finally, because of the absent changes in cycling behaviour, essential changes in accident and conflict occurrence are not verifiable.

Relevant for the choice of using the cycle path or the carriageway is the high weighting of cycling safety in combination with the high subjective safety sensibility on the cycle path, which was identified by the on-site road user interviews. Moreover, the configurations of traffic regulation regarding the obligation of cycle path usage are mostly unknown by road users. Therefore, these regulations are not crucial for the behaviour of cyclists.

In conclusion, this research shows no changes in cycling behavior as well as for the accident and conflict occurrence with the suspension of the obligation of cycle path usage, when no or just simple accompanying measures (pictograms) were implemented. Changes are noticeable, when direct offerings for cycling on the carriageway are realised (marking of cycle lanes or advisory lanes on the carriageway including the removal of the former cycle path). The general obligation of cycle path usage alone shows no effects on cycling behaviour and the safety of cycling lanes. In general, cycling lanes should provide a high design standard unattached by the obligation of its usage. Moreover, road safety management should defuse the relevant and already known accident and conflict hot spots. In this way, it is possible to meet the general and safety related requirements of the improving cycling traffic. 


\section{INHALTSVERZEICHNIS}

1

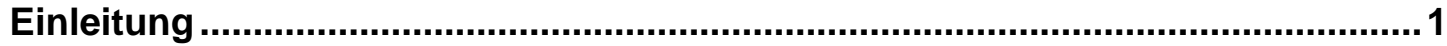

Literaturanalyse ....................................................................................................... 3

2.1 Verkehrsrechtliche Grundlagen in Deutschland................................................... 3

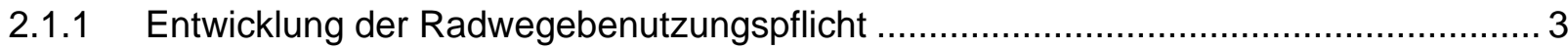

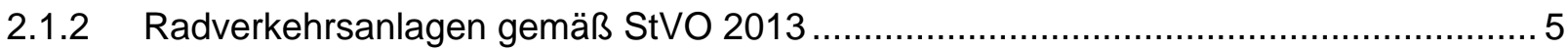

2.1.3 Anordnung der Radwegebenutzungspflicht gemäß StVO 2013 ............................... 6

2.2 Verkehrsrechtliche Regelungen außerhalb Deutschlands ...................................... 7

2.2.1 Schweiz

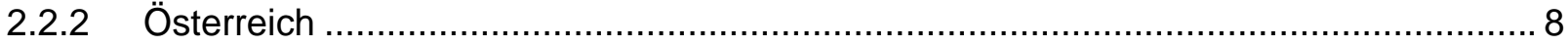

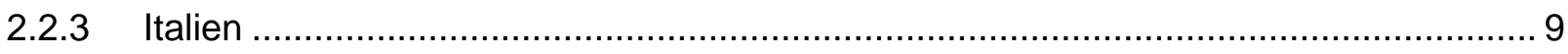

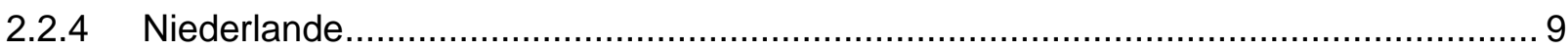

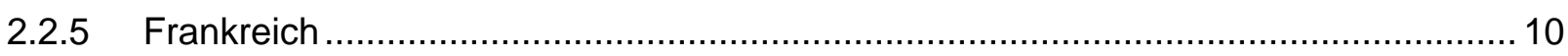

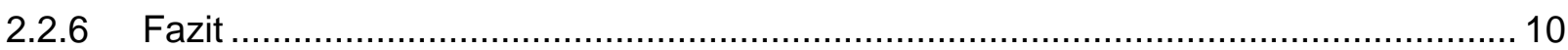

2.3 Empfehlungen für Radwege in den deutschen Planungsregelwerken...................... 11

2.3.1 Vorauswahl von Führungsformen des Radverkehrs ........................................ 12

2.3.2 Realisierbarkeit von Radverkehrsführungen ..................................................... 13

2.3.3 Vergleich und Bewertung verschiedener Führungsformen ...................................... 14

2.3.4 Weitere Einflussgrößen auf die Führungsformen des Radverkehrs ......................... 15

2.4 Weitere Empfehlungen und Leitfäden ...................................................................

2.5 Erfahrungen zu Radverkehrsführungsformen ................................................... 19

2.5.1 Verkehrssicherheit und Konfliktsituation ...................................................... 20

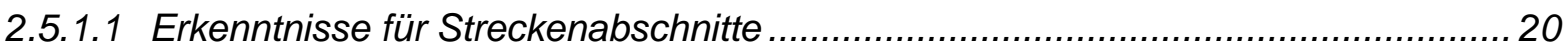

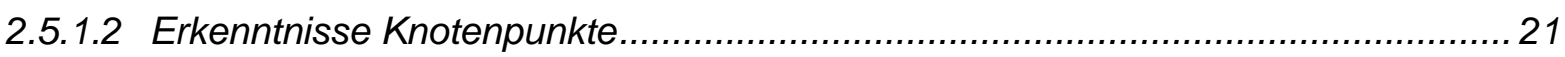

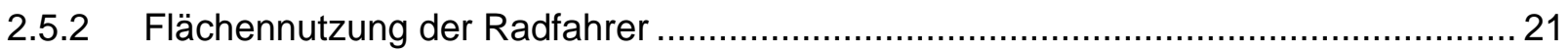

2.5.3 Maßnahmen zur Verbesserung der Radverkehrssicherheit ..................................... 23

2.5.4 Schlussfolgerungen aus vorhandenen Untersuchungen ....................................... 24

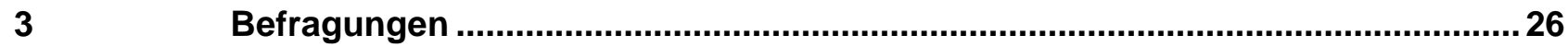

3.1 Erste Umfragephase (Onlineumfrage) …..................................................... 26

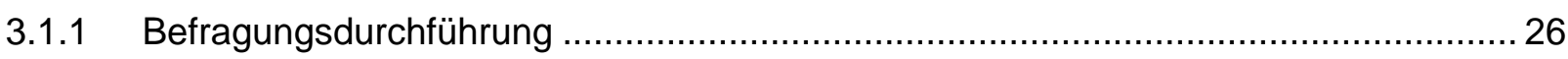

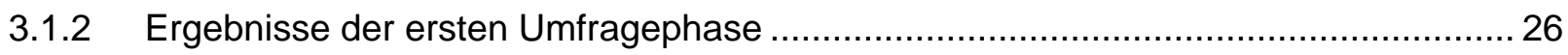

3.1.2.1 Status der kommunalen Radwegebenutzungspflicht.....................................26

3.1.2.2 Begleit- und Unterhaltungsmaßnahmen ..................................................... 30

3.1.2.3 Auswirkungen der Aufhebung der Radwegebenutzungspflicht ............................. 34

3.1.2.4 Anordnungskriterien der Radwegebenutzungspflicht ..................................... 40

3.1.2.5 Bereitschaft zur zweiten Umfragephase .......................................................... 43

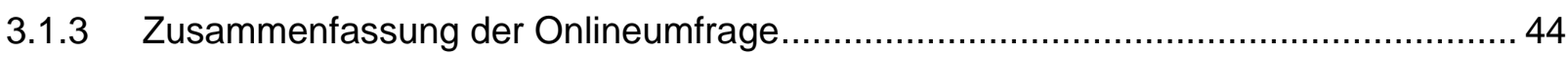

3.2 Städteauswahl für die weitergehende Untersuchung ...........................................46

3.3 Definition von Typen nicht benutzungspflichtiger Radwege...................................46

3.4 Zweite Umfragephase (Telefonbefragung) ........................................................ 47 


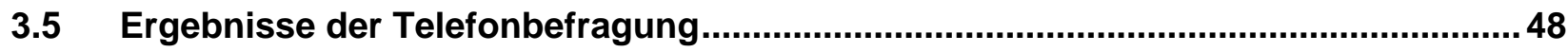

$4 \quad$ Methodik der Unfall- und Verkehrsverhaltensuntersuchung ..............................49

4.1 Makroskopische Unfallanalyse.............................................................................. 49

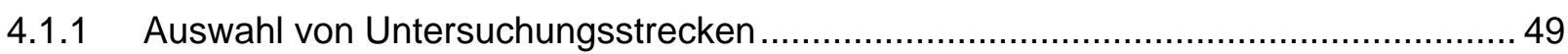

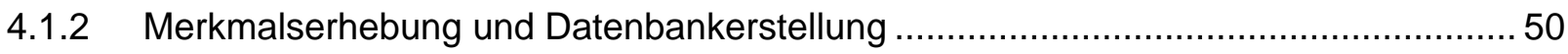

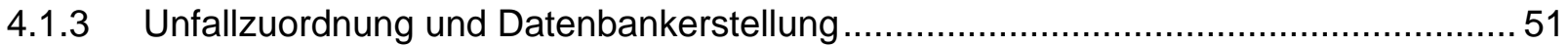

4.1.4 Sensitivitätsanalyse des Untersuchungskollektives ............................................. 52

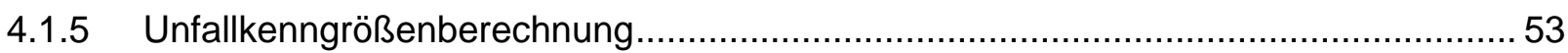

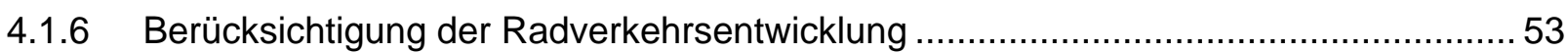

4.1.7 Bestandteile der makroskopischen Unfallanalyse ................................................ 54

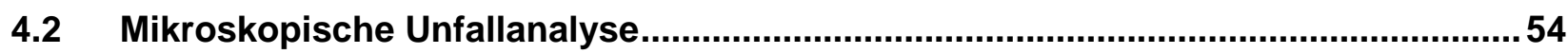

4.3 Vorher-Nachher-Untersuchung des Verkehrsverhaltens...........................................56

4.3.1 Auswahl von benutzungspflichtigen Radwegen................................................. 56

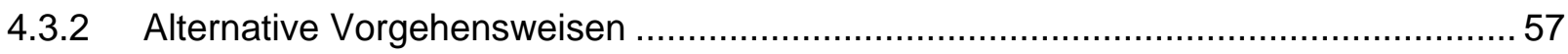

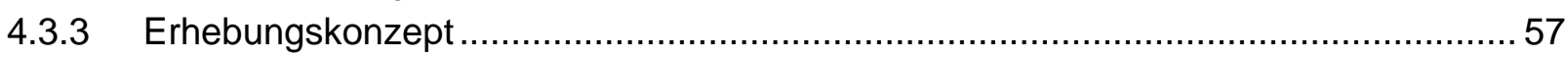

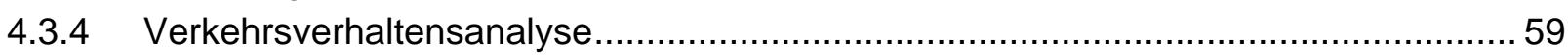

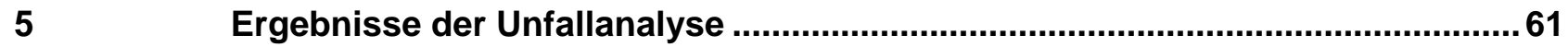

5.1 Allgemeine Tendenzen im Unfallgeschehen ....................................................61

5.2 Unfallentwicklung im Untersuchungskollektiv und Sensitivitätsanalyse ................62

5.3 Veränderungen im Radunfallgeschehen ...............................................................64

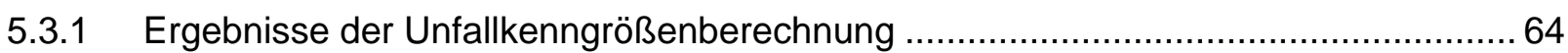

5.3.1.1 Unfallkenngrößen ohne Projektion der Radverkehrsstärken ..............................6 64

5.3.1.2 Unfallkenngrößen mit Projektion der Radverkehrsstärken ..................................64

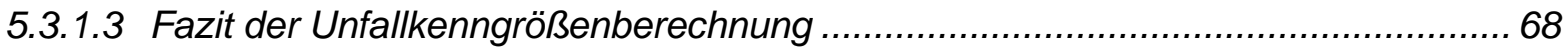

5.3.2 Exkurs: Flächennutzung der Radfahrer an nicht benutzungspflichtigen Radwegen ... 70

5.3.3 Ergebnisse der makroskopischen und mikroskopischen Unfallanalyse .................... 72

5.3.3.1 Radunfallgeschehen nach Begleitmaßnahmen............................................ 72

5.3.3.2 Radunfallgeschehen an Radwegen ohne Begleitmaßnahmen nach allgemeinen

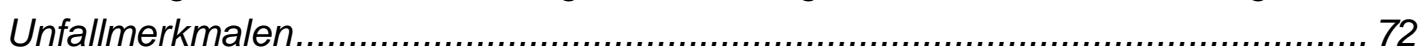

5.3.3.3 Radunfallgeschehen unter Berücksichtigung der Unfallbeteiligten........................ 75

5.3.3.4 Fazit der makroskopischen und mikroskopischen Unfallanalyse ........................ 77

$6 \quad$ Ergebnisse der Verkehrsverhaltensanalyse ..................................................... 79

6.1 Erkenntnisse zur Flächennutzung ....................................................................... 79

6.1.1 Radverkehrsanlagen der Vorher-Nachher-Vergleiche ...................................... 79

6.1.2 Radverkehrsanlagen der Mit-Ohne-Vergleiche ................................................... 80

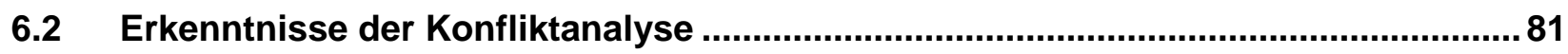

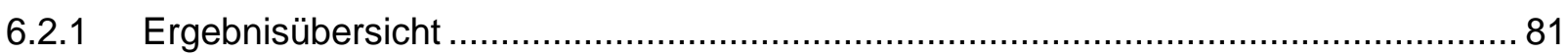

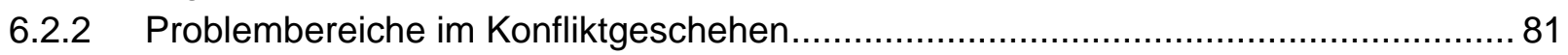

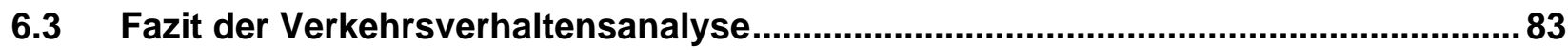

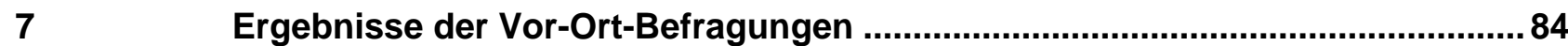




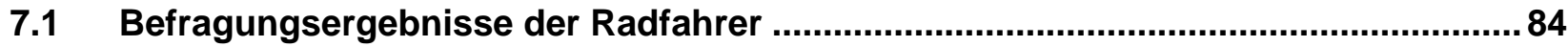

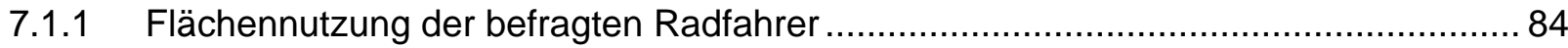

7.1.2 Bewertung der Interaktionen mit anderen Verkehrsteilnehmern............................... 86

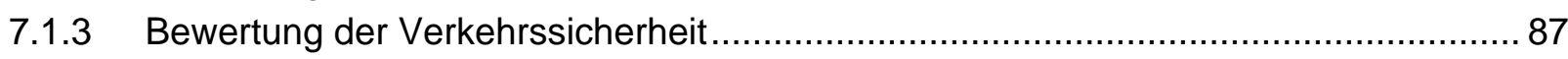

7.2 Befragungsergebnisse der Fußgänger und Autofahrer ......................................... 88

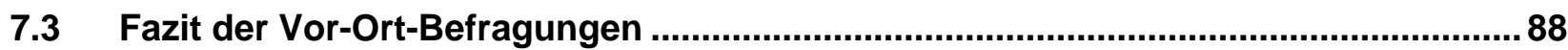

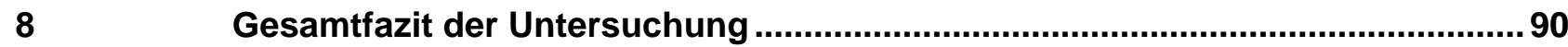

8.1 Kurzzusammenfassung der Projektergebnisse ..................................................... 90

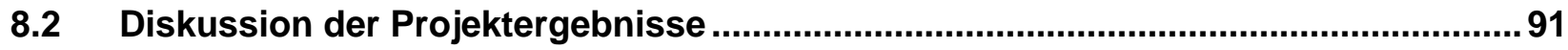

8.3 Empfehlungen zur Radwegebenutzungspflicht .................................................. 93

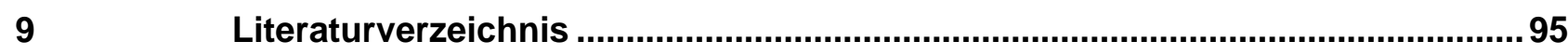

Anhang A Verkehrszeichen für Radwege in den untersuchten europäischen Staaten....100

Anhang B Empfehlungen zur Radwegebenutzungspflicht nach Mainzer Leitfaden......... 103

Anhang C Unfallkenngrößen ohne Projektion der Radverkehrsstärken...........................104

Anhang D Unfallkenngrößen mit Projektion der Radverkehrsstärken............................... 105

Anhang E Unfallrate und Unfallkostenrate nach Kraftfahrzeugverkehrsstärken an Radwegen mit aufgehobener Benutzungspflicht ohne Begleitmaßnahmen ... 106

Anhang F Unfallrate und Unfallkostenrate nach Radverkehrsstärken an Radwegen mit aufgehobener Benutzungspflicht ohne Begleitmaßnahmen.......................... 107

Anhang G Unfallrate und Unfallkostenrate nach Fahrstreifenanzahl an Radwegen mit aufgehobener Benutzungspflicht ohne Begleitmaßnahmen............................ 108

Anhang H Unfallrate und Unfallkostenrate nach Breite des rechten Fahrstreifens an Radwegen mit aufgehobener Benutzungspflicht ohne Begleitmaßnahmen ... 109

Anhang I Radunfallgeschehen nach Begleitmaßnahmen............................................. 110

Anhang J Radunfallgeschehen nach Unfallkategorien.................................................... 111

Anhang K Radunfallgeschehen nach Unfalltypen .......................................................... 113

Anhang $L \quad$ Radunfallgeschehen nach Hauptunfallverursacher ..................................... 115 


\section{ABBILDUNGSVERZEICHNIS}

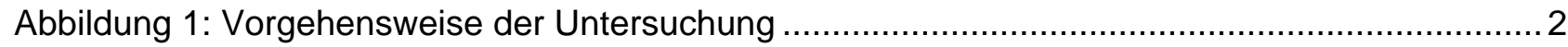

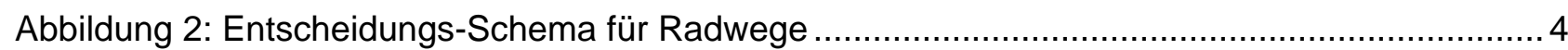

Abbildung 3: Radwegebenutzungspflicht anordnende Verkehrszeichen gemäß StVO .................5

Abbildung 4: Verkehrszeichen für Radwege ohne Benützungspflicht in Österreich ..................... 8

Abbildung 5: Kennzeichnung nicht benutzungspflichtiger Radwege in den Niederlanden..............9

Abbildung 6: Belastungsbereiche und zugeordnete geeignete Radverkehrsführungen an zweiund vierstreifigen Stadtstraßen nach ERA ..................................................... 12

Abbildung 7: Verkehrsräume und lichte Räume des Radverkehrs nach RASt ..........................13

Abbildung 8: Ermittlung erforderlicher Radverkehrsanlagen .............................................. 18

Abbildung 9: Subjektive Sinnhaftigkeit der Aufhebung der Radwegebenutzungspflicht ...............27

Abbildung 10: Existenz nicht benutzungspflichtiger Radwege in den Städten ............................2 27

Abbildung 11: Status der Überprüfung der Benutzungspflicht der Radwege in den Städten ..........28

Abbildung 12: Ursachen des Handlungserfordernisses zur Überprüfung der Radwege ................29

Abbildung 13: Status der Aufhebung der Radwegebenutzungspflicht in den Städten auf Basis der Radwegeüberprüfungen.

Abbildung 14: Begleitmaßnahmen der Städte mit Aufhebung der Radwegebenutzungspflicht .......31

Abbildung 15: Regelmäßig durchgeführte Maßnahmen an Radwegen mit und ohne Benutzungspflicht

Abbildung 16: Bewertung der Konflikthäufigkeit und der Verkehrssicherheit vor und nach der Aufhebung der Radwegebenutzungspflicht............................................... 34

Abbildung 17: Positive Effekte durch die Aufhebung der Radwegebenutzungspflicht ....................35

Abbildung 18: Negative Effekte durch die Aufhebung der Radwegebenutzungspflicht .................37

Abbildung 19: Häufigkeit der Fahrbahnnutzung der Radfahrer bei nicht benutzungspflichtigen

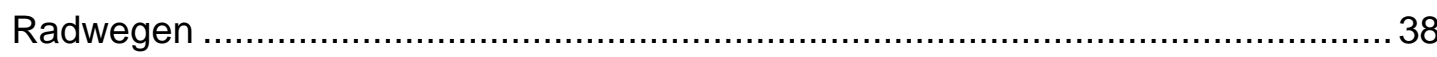

Abbildung 20: Akzeptanz der Kraftfahrzeugführer gegenüber den Radfahrern auf der Fahrbahn bei nicht benutzungspflichtigen Radwegen

Abbildung 21: Kriterien zur Beurteilung der Gefahrenlage bei Entscheidung über die Benutzungspflicht der Radwege.

Abbildung 22: Kriterien zur Beurteilung der Benutzungspflicht von Radwege aufgrund der Verkehrsqualität

Abbildung 23: Herstellung des Zeitbezuges zwischen Unfalldatum und Zeitpunkt der Aufhebung der Radwegebenutzungspflicht .

Abbildung 24: Auswerteschema der makroskopischen und mikroskopischen Unfallanalyse .........55

Abbildung 25: Schema des Erhebungsdesigns für die Verkehrsverhaltensanalysen ...................58

Abbildung 26: Systematik der Auswertungen zur Verkehrsverhaltensanalyse ............................59

Abbildung 27: Unfallentwicklung mit Aufhebung der Radwegebenutzungspflicht....................... 61 
Abbildung 28: Unfallentwicklung differenziert nach knotenpunktfreier Strecke und Knotenpunkten

Abbildung 29: Veränderungen im Radunfallgeschehen auf einzelnen Untersuchungsstrecken.....

Abbildung 30: Entwicklung des Quotienten aus Rad- und Gesamtunfallgeschehen auf den betrachteten Untersuchungstrecken, in den ausgewählten Städten sowie in Gesamtdeutschland 63

Abbildung 31: Unfallraten an knotenpunktfreier Strecke nach Begleitmaßnahmen 65

Abbildung 32: Unfallraten an Knotenpunkten nach Begleitmaßnahmen 66

Abbildung 33: Nutzung der Fahrbahn von Radfahrern an nicht benutzungspflichtigen Radwegen 70

Abbildung 34: Nutzung der Fahrbahn von Radfahrern in Abhängigkeit des Kraftfahrzeugverkehrs an nicht benutzungspflichtigen Radwegen ohne weitere Begleitmaßnahmen

Abbildung 35: Verschiebungen in der Flächennutzung bei Radunfällen auf der knotenpunktfreien Strecke nach Unfalltypen

Abbildung 36: Verschiebungen in der Flächennutzung bei Radunfällen an Knotenpunkten nach Unfalltypen

Abbildung 37: Unfallgeschehen und Fahrverhalten von Radfahrern auf der knotenpunktfreien Strecke differenziert nach Flächennutzung und Kraftfahrzeugverkehrsstärken 75

Abbildung 38: Konfliktgeschehen beim Radwegfahren an Knotenpunkten.

Abbildung 39: Konfliktgeschehen beim Radwegfahren auf knotenpunktfreier Strecke 82

Abbildung 40: Gründe für die Radwegnutzung durch Radfahrer 85

Abbildung 41: Bedeutung von Sicherheit und Vorankommen für die Wahl der Flächennutzung.... 85

Abbildung 42: Wahrgenommene Beeinträchtigungen und Konflikte 86

Abbildung 43: Sicherheitsbewertung für das Fahren auf der knotenpunktfreien Strecke 87

Abbildung 44: Sicherheitsbewertung für das Fahren an Knotenpunkten 87 


\section{TABELLENVERZEICHNIS}

Tabelle 1: Erforderliche Breiten zur Anordnung einer Radwegebenutzungspflicht nach VwVStVO

Tabelle 2: Mögliche Fälle geprüfter Radverkehrsanlagen und Notwendigkeit geeigneter Maßnahmen nach Mainzer Leitfaden 18

Tabelle 3: Clusterung von nicht benutzungspflichtigen Radwegen

Tabelle 4: Ausgewählte Radwege ohne Benutzungspflicht nach Städten und Begleitmaßnahmen 50

Tabelle 5: Verwendete angepasste Unfallkostensätze 53

Tabelle 6: Abschätzung der Radverkehrsentwicklung in den betrachteten Städten 65

Tabelle 7: Umgerechnete richtungsbezogene Tagesverkehrsstärken in Anlehnung an ERA..... 67

Tabelle 8: Ergebnisübersicht zur Verkehrsverhaltensanalyse 79

Tabelle 9: Flächennutzung vor und nach Aufhebung der Radwegebenutzungspflicht bei der Begleitmaßnahme Radfahrstreifen und Rückbau Radweg .79

Tabelle 10: Flächennutzung vor und nach Aufhebung der Radwegebenutzungspflicht bei der Begleitmaßnahme Radfahrerpiktogramme.

Tabelle 11: Flächennutzung an 4 Radwegen mit ähnlicher Streckencharakteristik differenziert nach Radwegebenutzungspflicht (Aufhebung ohne weitere Begleitmaßnahmen) ....... 80

Tabelle 12: Ergebnisübersicht zum Konfliktgeschehen nach Streckenelementen 81

Tabelle 13: Übersicht der auswertbaren Fragebögen 84 


\section{ABKÜRZUNGSVERZEICHNIS}

DTV

durchschnittliche tägliche Verkehrsstärke

EFA

Empfehlungen für Fußgängerverkehrsanlagen

ERA

Empfehlungen für Radverkehrsanlagen

FGSV

Forschungsgesellschaft für Straßen- und Verkehrswesen

$\mathrm{Kfz}$

Kraftfahrzeug

LSA

Lichtsignalanlage

RASt

Richtlinien für die Anlage von Stadtstraßen

StVO

Straßenverkehrs-Ordnung

VwV-StVO

Allgemeine Verwaltungsvorschrift zur Straßenverkehrs-Ordnung 


\section{Einleitung}

Die verkehrsrechtlichen Regelungen zur Benutzungspflicht von Radwegen unterlagen in Deutschland in den vergangenen Jahren einem starken Wandel. Nach den ausschließlich benutzungspflichtigen Radwegen in den 1970er Jahren wurde durch die StVO Novelle von 1997 erstmals die generelle Radwegebenutzungspflicht aufgehoben und Radwege mit Benutzungsrecht ermöglicht. Seit der StVO Novelle von 2009, mehrerer anschließender Gerichtsurteile und mündend in der Neufassung der StVO von 2013 ist nunmehr das Verbot der Straßennutzung und somit die Ausweisung einer Radwegebenutzungspflicht gemäß StVO nur noch dann gerechtfertigt, „wenn auf Grund der besonderen örtlichen Verhältnisse eine Gefahrenlage besteht, die das allgemeine Risiko [...] erheblich übersteigt“ sowie wenn es nach VwV-StVO „die Verkehrssicherheit oder der Verkehrsablauf erfordern".

Ziel des vorliegenden Forschungsvorhabens ist es, die Veränderungen der Verkehrssicherheit bei Aufhebung der Radwegebenutzungspflicht zu evaluieren. Zudem sollen Randbedingungen identifiziert werden, bei denen die Sicherheit für den Radverkehr aber auch die der übrigen Verkehrsteilnehmer ungünstig beeinflusst wird und die Einrichtung einer benutzungspflichtigen Radverkehrsanlage aus Sicherheitsgründen zu empfehlen wäre.

Die Untersuchung gliedert sich in die Arbeitsschritte gemäß Abbildung 1. Nach einer Literaturanalyse zur Thematik erfolgt zu Projektbeginn eine repräsentative Online-Befragung in deutschen Städten mit mehr als 20.000 Einwohnern. Dabei werden erfahrungsbasierte Erkenntnisse zum Umgang der Kommunen mit der Radwegebenutzungspflicht zusammengetragen und geeignete Untersuchungsbeispiele für das vorliegende Forschungsvorhaben identifiziert.

Nach einer Auswahl von nicht benutzungspflichtigen Radwegen für die entsprechenden Unfallanalysen erfolgen Erhebungen der infrastrukturellen, betrieblichen und verkehrlichen Randbedingungen an den Untersuchungsbeispielen. Weiterhin wird nach einer Zuordnung der Radunfälle der Kategorien 1 bis 3 eine Unfallkenngrößenberechnung unter Berücksichtigung der Radverkehrsentwicklung sowie eine kombinierte makroskopische und mikroskopische Unfallanalyse jeweils für den Zeitraum vor bzw. nach Aufhebung der Radwegebenutzungspflicht durchgeführt. Dabei gilt es, die Veränderungen im Radunfallgeschehen mit Aufhebung der Radwegebenutzungspflicht zu bewerten. Die Unfallanalyse konzentriert sich weiterhin auf die Sicherheitswirkungen verschiedener infrastruktureller und betrieblicher Eigenschaften der untersuchten Streckenabschnitte und Knotenpunkte und soll für die Gestaltung von nicht benutzungspflichtigen Radwegen entsprechende Empfehlungen ermöglichen.

Den zweiten Ansatz zur Prüfung der Sicherheitswirkungen bildet eine Verkehrsverhaltensbeobachtung an 10 ausgewählten Radwegen mit und 10 Radwegen ohne Benutzungspflicht, die durch eine Vor-Ort-Befragung von Verkehrsteilnehmern ergänzt wird. Dabei sollen kritische Verkehrsverhaltensweisen identifiziert werden, die rein aus den Unfalldaten nicht ableitbar sind. Die Verhaltensbeobachtung soll zudem Aufschluss über die tatsächliche Inanspruchnahme der Radverkehrsführungsformen geben. Ziel der Verhaltensbeobachtungen ist es analog zu den Vorher-/NachherUntersuchungen im Unfallgeschehen herauszufinden, inwiefern eine Nutzung oder Nichtnutzung von nicht benutzungspflichtigen Radwegen zu neuen Konflikten führt.

Aufbauend auf den gewonnenen Erkenntnissen beider beschriebenen Ansätze werden abschließend Hinweise aus Sicht der Verkehrssicherheit für kommunale Planungsträger zur Aufhebung der Benutzungspflicht von Radverkehrsanlagen erarbeitet, um die Sicherheit des städtischen Radverkehrs erhöhen zu können. 


\section{Untersuchungsablauf}

\section{Literaturanalyse}

\section{Befragung von Kommunen}

- Online-Befragung in 679 deutschen Städten mit mehr als 20.000 Einwohnern

- Ermittlung erfahrungsbasierter Erkenntnisse zum Umgang mit der Radwegebenutzungspflicht

- Telefonbefragung in ausgewählten Städten zur Identifizierung von Untersuchungsbeispielen

\section{Bestandsaufnahme}

- Auswahl nicht benutzungspflichtiger Radwege als Grundlage der folgenden Unfallanalysen

- Erhebung infrastruktureller, betrieblicher und verkehrlicher Randbedingungen

\section{Unfallanalyse}

- Zuordnung der Radverkehrsunfälle der Unfallkategorien 1-3

- Unfallkenngrößenberechnung unter Berücksichtigung der Radverkehrsentwicklung

- Kombinierte makroskopische und mikroskopische Unfallanalyse

- Bewertung des Radunfallgeschehens vor und nach Aufhebung der Radwegebenutzungspflicht

- Beurteilung des Einflusses infrastruktureller und betrieblicher Radwegeigenschaften

\section{Verkehrsverhaltensanalyse und Vor-Ort-Befragung}

- Verkehrsbeobachtungen an 10 Radwegen mit und 10 Radwegen ohne Benutzungspflicht

- Befragungen von Radfahrern, Fußgängern und Autofahrern an den betrachteten Radwegen

- Bewertung von Flächennutzung und Konfliktgeschehen der Radfahrer

\section{Zusammenführung der Ergebnisse und Empfehlungen}

\section{Abbildung 1: Vorgehensweise der Untersuchung}

Der vorliegende Bericht beinhaltet die finalen Projektergebnisse gemäß den zuvor geschilderten Arbeitsschritten und bildet den Abschluss des Projektes „Aufhebung der Benutzungspflicht von Radwegen". 


\section{Literaturanalyse}

Im Rahmen der nachfolgenden Literaturanalyse werden die vorhandenen Erkenntnisse zur Radwegebenutzungspflicht zusammengetragen und ausgewertet. Neben den verkehrsrechtlichen Grundlagen in Deutschland und einigen benachbarten Staaten werden hierbei die Vorgaben einschlägiger Richtlinien herausgearbeitet sowie die Erfahrungen von Kommunen und bereits durchgeführter Forschungsvorhaben zur Radwegebenutzungspflicht einbezogen.

\subsection{Verkehrsrechtliche Grundlagen in Deutschland}

\subsubsection{Entwicklung der Radwegebenutzungspflicht}

Die in der gegenwärtig gültigen Neufassung der StVO von 2013 enthalten Regelungen für die Radwegebenutzungspflicht unterlagen in den vergangenen Jahren einem starken Wandel. Besonders die vorherige Fassung der StVO vom 16. November 1970 wurde hierbei durch mehrere Änderungsverordnungen mit der Zeit stark angepasst. Seit der ersten Fassung von 1970 mussten Radfahrer demzufolge noch „rechte Seitenstreifen benutzen, wenn sie Fußgänger nicht behindern“ (StVO 1970, § 2, Absatz 2, Satz 2). Zusammen mit der Trennung von Kraftfahrzeug- und Radverkehr früherer Leitbilder der städtischen Verkehrsplanung entstand somit in der Vergangenheit eine Vielzahl baulicher, benutzungspflichtiger und „autogerechter Radwege“ [Horn 2002].

Die damit entstandenen umfangreichen Radwegesysteme genügten aber ab den 1990er Jahren nicht mehr den Erfordernissen des nun moderner gewordenen Radverkehrs. Viele Radwege befanden sich in baulich unzureichendem Zustand, waren zu schmal und wurden an Knotenpunkten nach veralteten Entwurfsprinzipien geführt. Weiterhin hatten sich „die Erwartungen in die Sicherheit als trügerisch erwiesen“ [Alrutz et al. 1998].

Für Radverkehrsanlagen die nicht den aktuellen Verkehrssicherheitsstandards unterlagen und nicht eine entsprechende Benutzungsqualität aufwiesen, wurde mit der StVO-Novelle von 1997 die generelle Benutzungspflicht von 1970 erstmals aufgehoben. Damit waren nur Radwege mit entsprechender Beschilderung benutzungspflichtig, auf allen anderen Radwegen galt ein Nutzungsrecht [Alrutz et al. 1998]. Für die Aufnahme der entsprechenden Neuregelungen in die damalige StVO musste aber auch im Vorfeld intensive Überzeugungsarbeit zu deren Notwendigkeit und Richtigkeit geleistet werden [Draeger 1997].

Zur Überprüfung der Anordnungen im Radwegenetz waren generelle Prüfungen des Erfordernisses der Radwegenutzungspflicht (basierend auf der Verkehrssicherheit), anschließend Prüfungen der Anforderungen an Radwege (Breite, Beschaffenheit, Linienführung) und abschließend Entscheidungen basierend auf beiden Kriterien sowie potentiellen Verbesserungsmaßnahmen zur Herstellung der Grundanforderungen an einen Radweg notwendig. Für die Umsetzung der Prüfungen im Radwegenetz wurde den Straßenverkehrsbehörden eine Übergangsfrist bis zum 01.10.1998 gewährt [Alrutz et al. 1998]. Dazu zeigt Abbildung 2, dass auch die Beibehaltung der Radwegebenutzungspflicht ohne deren tatsächliches Erfordernis möglich war (Fall 6).

Für die Beurteilung einer Benutzungspflicht aus Gründen der Verkehrssicherheit waren verschiedene Handlungsempfehlungen vorhanden. Entfallen konnte die Benutzungspflicht bei Tempo-30Zonen und Kraftverkehrsstärken unter $5.000 \mathrm{Kfz} / 24 \mathrm{~h}$. Zwischen 5.000 und $10.000 \mathrm{Kfz} / 24 \mathrm{~h}$ und hohem Schwerverkehrsanteil, hohem Geschwindigkeitsniveau oder hoher Kurvigkeit konnte eine Benutzungspflicht angeordnet werden, ab $10.000 \mathrm{Kfz} / 24 \mathrm{~h}$ (bei $50 \mathrm{~km} / \mathrm{h}$ ) wurde diese als erforderlich und ab $20.000 \mathrm{Kfz} / 24 \mathrm{~h}$ (bei $50 \mathrm{~km} / \mathrm{h}$ ) sogar als unerlässlich angesehen [FGSV 1998]. 


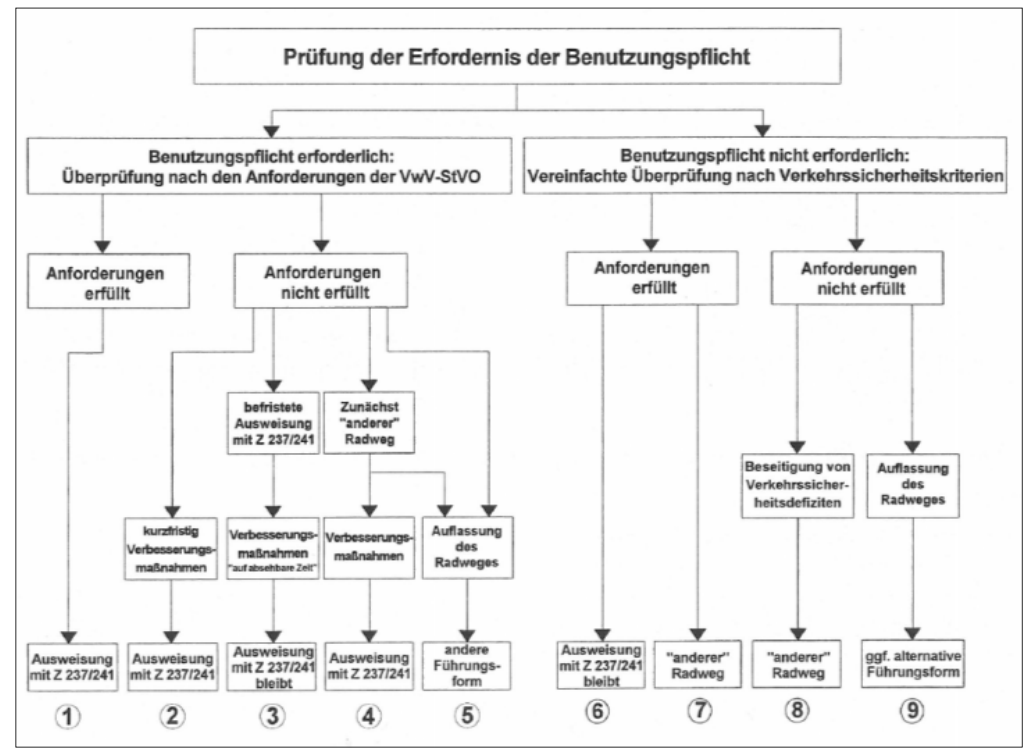

Abbildung 2: Entscheidungs-Schema für Radwege [Alrutz et al. 1998]

Die weiteren Anforderungen an benutzungspflichtige Radwege waren einzuhaltende Radwegbreiten gemäß VwV-StVO (gleich den Angaben der aktuellen Vorgaben der StVO), gute Radwegzustände (ohne starke Unebenheiten oder schlecht befahrbare Bordabsenkungen sowie Aufwölbungen/Aufbrüche durch Wurzelwerk), geeignete Linienführung auf der Strecke (optische Abgrenzung von angrenzenden Flächen durch Beläge oder Markierungen und keine Sichthindernisse) und an Knotenpunkten (gute Sichtbeziehungen, Erkennbarkeit und Gestaltung der Radwegführung) [Alrutz et al. 1998]. Zahlreiche der damaligen Radwege wiesen jedoch Mängel auf (u.a. Einbauten im Sicherheitsraum der Radfahrer, Schlaglöcher, ungenügend abgesenkte Borde) und führten oftmals zu Stürzen und somit zu Alleinunfällen [Draeger 1997]. Die damalige Praxis zur Überprüfung der Radwegebenutzungspflicht war jedoch auch „gekennzeichnet von Unsicherheiten bzw. zum Teil sehr unterschiedlichen Herangehensweisen" [Alrutz et al. 1998].

Zum Umgang mit Radwegen mit aufgehobener Benutzungspflicht („andere Radwege“) existierten drei Möglichkeiten: Zuführung einer anderen Nutzung, vorrübergehende Radwegstillegung oder Kennzeichnung als „anderer Radweg“ durch Fahrradpiktogramme. Jedoch sollte darauf geachtet werden, dass auch "andere Radwege“ die baulichen Voraussetzungen der Kennzeichnung einer Radwegebenutzungspflicht erfüllen [Draeger 1997].

Mit der 46. Änderung der StVO im Jahr 2009 sollte ein weiterer Beitrag zur Sicherheit des Radverkehrs geleistet werden. Hierzu sollten die Radverkehrsvorschriften gestrafft und vereinfacht sowie den Behörden größere Handlungsspielräume gegeben werden. Seit 1997 wurden die dort eingeführten rechtlichen Vorgaben von der Mehrzahl der Anordnungsbehörden ignoriert. Mit der 2009er Novelle sollten die Anforderungen an einen benutzungspflichtigen Radweg weiter erhöht werden. Durch diese neue Novelle sollten die aus der vergangenen Novelle hervorgegangenen zu unkritischen Anordnungen einer Radwegebenutzungspflicht nun auf die Verkehrssicherheit konzentriert und auf das tatsächlich erforderliche Maß reduziert werden [Stephan et al. 2010].

Die Anordnung einer Radwegebenutzungspflicht war hier nur aus Gründen der Verkehrssicherheit als Grundvoraussetzung erforderlich und weiterhin erst bei Erfüllung der geforderten Mindestbreiten von Radwegen vorzunehmen. Bei entsprechender Anordnung mussten aber auch die Belange der Fußgänger beachtet werden. Weiterhin mussten die „anderen Radwege“ nun nicht mehr die baulichen Anforderungen eines benutzungspflichtigen Radweges erfüllen, da nur die verkehrssichere Führung für benutzungspflichtige Radwege im Vordergrund stand. Dennoch sollten nicht 
benutzungspflichtige Radwege als gleichwertige und qualitativ hochwertige Radverkehrsanlagen und daher nicht als Radwege 2. Klasse angesehen werden. Die Anordnung Gehweg Radfahrer frei erforderte weiterhin eine Oberflächenbeschaffenheit entsprechend der Radverkehrsbedürfnisse. Erstmalig wurde in dieser StVO explizit auf die Empfehlungen für Radverkehrsanlagen (ERA) verwiesen [Stephan et al. 2010].

In einer Pressemitteilung vom 13.04.2010 erklärte das BMVBS die StVO-Novelle von 2009 aufgrund von Verstößen gegen ein verfassungsrechtliches Zitiergebot für nichtig [BMVBS 2010]. Zur Vermeidung weiterer derartiger Verstöße wurde eine Neufassung der StVO beschlossen, in der auch die Errungenschaften der "Schilderwaldnovelle" von 2009 eingebunden werden sollten [BMVBS 2012]. Die aktuell gültigen Regelungen der StVO Neufassung von 2013 sind in nachfolgendem Kapitel dargestellt.

\subsubsection{Radverkehrsanlagen gemäß StVO 2013}

In $\S 2$ der Neufassung der Straßenverkehrsordnung ist grundlegend die Straßenbenutzung durch Fahrzeuge geregelt. „Eine Pflicht, Radwege in der jeweiligen Fahrtrichtung zu benutzen, besteht [gemäß § 2, Absatz 4, Satz 2] nur, wenn dies durch Zeichen 237, 240 oder 241 angeordnet ist“ [StVO 2013]. Derart benutzungspflichtige Radwege unterscheiden sich entsprechend Abbildung 3 in baulich angelegte Radwege und Radfahrstreifen (Z 237 StVO), gemeinsame Geh- und Radwege (Z 240 StVO) und die für den Radverkehr bestimmten Teile von getrennten Geh- und Radwegen (Z 241 StVO) [VwV-StVO 2015]. Das Ende eines dieser Wege kann durch das Zusatzzeichen „Ende“ angezeigt werden. Ist das Ende der Wege allerdings zweifelsfrei erkennbar, entfällt diese Kennzeichnung (VwV-StVO zu Z 237, 240 und 241).

Gemeinsame Geh- und Radwege kommen nur in Betracht, wenn die Belange der Fußgänger berücksichtigt sowie die Sicherheit und Leichtigkeit des Radverkehrs nicht beeinträchtigt werden und die Beschaffenheit der Verkehrsfläche den Anforderungen des Radverkehrs genügt (VwV-StVO zu Z 240). Weiterhin können Gehwege für Radfahrer mit dem Zusatzzeichen „Radfahrer frei“ geöffnet werden (VwV-StVO zu Z 239). „Kinder bis zum vollendeten achten Lebensjahr müssen, ältere Kinder bis zum vollendeten zehnten Lebensjahr dürfen mit Fahrrädern [dagegen grundsätzlich] Gehwege benutzen" (StVO § 2, Absatz 5). Für die Anordnung eines getrennten Geh- und Radweges sind ebenfalls die Belange der Fußgänger zu berücksichtigen. Weiterhin ist hier auf eine eindeutige Zuordnung der Verkehrsflächen beider Verkehrsteilnehmer zu achten (VwV-StVO zu Z 241).

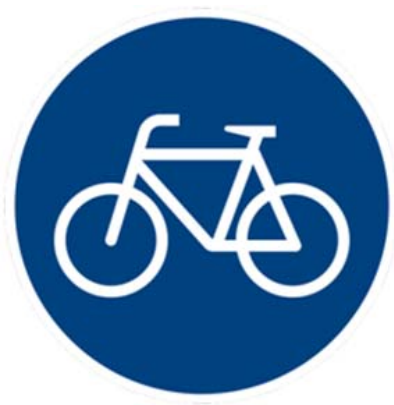

Z 237 „Radweg“

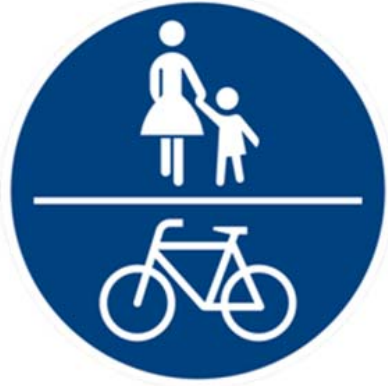

Z 240 ,gemeinsamer Geh- und Radweg“

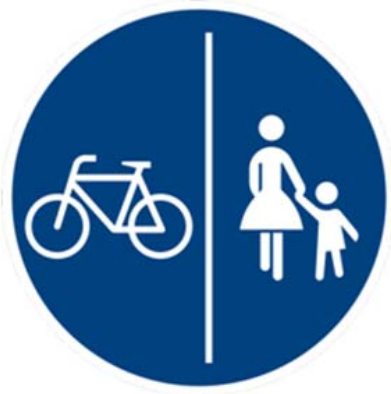

Z 241 ,getrennter Geh- und Radweg“"

Abbildung 3: Radwegebenutzungspflicht anordnende Verkehrszeichen gemäß StVO

Rechte Radwege einer Straße ohne die Zeichen 237, 240 oder 241 dürfen benutzt werden (vgl. StVO § 2, Absatz 4, Satz 3), linke Radwege ohne die Zeichen 237, 240 oder 241 dagegen nur, wenn es durch das Zusatzzeichen „Radverkehr frei“ angezeigt ist (vgl. StVO § 2, Absatz 4, Satz 4). 
Neben Radwegen im Straßenseitenraum existieren auch Sonderwege für Radfahrer. Dabei handelt es sich um - mit Zeichen 237 StVO gekennzeichnete und durch Zeichen 295 StVO (durchgezogene Fahrbahnbegrenzungslinie) von der Fahrbahn des Kraftfahrzeugverkehrs abgetrennte Radfahrstreifen. Zur Verdeutlichung des Radfahrstreifens kann im Streckenverlauf in regelmäßigen Abständen Zeichen 237 StVO markiert werden. Ist die Anlage eines Radfahrstreifens wegen geringer verfügbarer Breiten nicht möglich, kann ein Schutzstreifen mit Z 340 StVO (unterbrochene Leitlinie) markiert und mit dem Sinnbild „Fahrräder" verdeutlicht werden. Dieser muss einen hinreichenden Bewegungsraum für die Radfahrer aufweisen und das gefahrlose Begegnen zweier Personenkraftwagen im verbleibenden Teil der Fahrbahn ermöglichen. Weiterhin darf die Verkehrszusammensetzung eine Mitbenutzung des Schutzstreifens durch Kraftfahrzeuge nur selten erforderlich machen (vgl. VwV-StVO zu § 2, Absatz 4, Satz 2). Zur konkreten Gestaltung der Radverkehrsanlagen wird seitens der Verwaltungsvorschriften der Straßenverkehrsordnung auf die aktuell gültigen Empfehlungen für Radverkehrsanlagen - gegenwärtig ERA [2010] - verwiesen.

\subsubsection{Anordnung der Radwegebenutzungspflicht gemäß StVO 2013}

„Straßenverkehrsbehörden können die Benutzung bestimmter Straßen oder Straßenstrecken aus Gründen der Sicherheit oder Ordnung des Verkehrs beschränken oder verbieten“ (StVO § 45, Absatz 1, Satz 1). In Hinblick auf die Verkehrssicherheit „dürfen insbesondere Beschränkungen und Verbote des fließenden Verkehrs nur angeordnet werden, wenn auf Grund der besonderen örtlichen Verhältnisse eine Gefahrenlage besteht, die das allgemeine Risiko [...] erheblich übersteigt" (StVO § 45, Absatz 9, Satz 2). Hierunter fällt nach einem Urteil des Bundesverwaltungsgerichtes auch die Radwegebenutzungspflicht [BVerwG Leipzig, Urteil vom 18.11.2010 - 3 C 42.09]. Dementsprechend ist für die Anordnung eines benutzungspflichtigen Radweges der Nachweis einer besonderen Gefahrenlage notwendig, was wiederum vermehrt zu einer weiteren Welle der Aufhebung der Radwegebenutzungspflicht in Deutschland führte. Fall 6 gemäß Abbildung 2 - also die Beibehaltung der Radwegebenutzungspflicht ohne deren eigentliches Erfordernis - existiert bei dieser Voraussetzung nicht mehr.

An die Anordnung einer Radwegebenutzungspflicht werden weiterhin in den Verwaltungsvorschriften zur Straßenverkehrsordnung verschiedene Anforderungen gestellt. Benutzungspflichtige Radwege dürfen nur dort angeordnet werden, wo für den Fußgängerverkehr weiterhin ausreichende Flächen zur Verfügung stehen und wo es die Verkehrssicherheit oder der Verkehrsablauf erfordern (VwV-StVO zu § 2, Absatz 4, Satz 2). Neben dem Grundsatz, so wenig Verkehrszeichen wie möglich anzuordnen, darf gemäß VwV-StVO zu §§ 39 bis 43 über die Anordnung von Verkehrszeichen nur nach entsprechender Prüfung entschieden werden. Grundlegend ist dabei die Verkehrssicherheit aller Verkehrsteilnehmer vor der Flüssigkeit des Verkehrs zu berücksichtigen.

Weitere Voraussetzung für die Anordnung eines benutzungspflichtigen Radweges ist das Vorhandensein ausreichender Verkehrsflächen. Außerdem muss „die Benutzung des Radweges nach der Beschaffenheit und dem Zustand zumutbar sowie die Linienführung [im Streckenverlauf, an Knotenpunkten und Eimündungen sowie verkehrsreichen Grundstückzufahrten] eindeutig, stetig und sicher" sein (VwV-StVO zu § 2, Absatz 4, Satz 2). Zudem muss der Radweg entsprechend des Stands der Technik gebaut und unterhalten werden.

Zur Erfüllung der genannten weiteren Voraussetzungen muss der potentielle Radweg „ausreichend breit, befestigt und einschließlich einem Sicherheitsraum frei von Hindernissen" sein (VwV-StVO zu § 2, Absatz 4, Satz 2). Für die in Kapitel 2.1.2 genannten verschiedenartigen Radverkehrsanlagen gelten hierzu die Breitenanforderungen nach Tabelle 1, von denen nur in Ausnahmefällen auf kurzer Länge (Engstellen) unter Aufrechterhaltung der Verkehrssicherheit abgewichen werden 
kann. Die angegebenen Breiten beziehen sich dabei nur auf einspurige Fahrräder. Übersteigt jedoch die Gefahrenlage auf der Fahrbahn die Gefährdung eines - nicht den Mindestanforderungen der VwV-StVO entsprechenden - Radweges, so kann bei zumutbarer Benutzung trotzdem eine Benutzungspflicht angeordnet werden [VGH Bayern, Urteil vom 06.04.2011 - 11 B 08.1892].

\begin{tabular}{|c|c|c|c|}
\hline Zeichen StVO & Beschreibung der Radverkehrsanlage & & Breite [m] \\
\hline \multirow{4}{*}{ Z 237} & \multirow{2}{*}{ Baulich angelegter Radweg } & möglichst & 2,00 \\
\hline & & mindestens & 1,50 \\
\hline & \multirow{2}{*}{ Radfahrstreifen (einschließlich Z 295) } & möglichst & 1,85 \\
\hline & & mindestens & 1,50 \\
\hline \multirow{2}{*}{ Z 240} & \multirow{2}{*}{ Gemeinsamer Geh- und Radweg } & innerorts & 2,50 \\
\hline & & außerorts & 2,00 \\
\hline Z 241 & Getrennter Geh- und Radweg (Radwegteil) & mindestens & 1,50 \\
\hline
\end{tabular}

Tabelle 1: Erforderliche Breiten zur Anordnung einer Radwegebenutzungspflicht nach VwV-StVO

Die Anordnung der Radwegebenutzungspflicht obliegt den Straßenverkehrsbehörden unter Anhörung von Straßenbaubehörde und Polizei. Städtische Radverkehrsplaner und Straßenbaulastträger sollten bei der Entscheidung ebenfalls einbezogen werden. Zudem sind Radverkehrsanlagen bei jeder sich bietenden Gelegenheit - auf deren Zweckmäßigkeit zu prüfen und bei Bedarf bauliche Maßnahmen bei der Straßenbaubehörde anzuregen (VwV-StVO zu § 2, Absatz 4, Satz 2).

Nicht benutzungspflichtige Radwege sind gemäß VwV-StVO grundsätzlich für Radverkehre vorgesehene Flächen. Hierbei ist vorrangig an Knotenpunkten und verkehrsreichen Grundstückszufahrten auf eine verkehrssichere Führung zu achten und die Fremdnutzung der Radwege durch ruhende Verkehre zu unterbinden. Eine Benutzungspflicht linksseitiger Radwege ist in Ausnahmefällen und nur nach sorgfältiger Prüfung anzuordnen, da vom Radverkehr in Gegenrichtung innerhalb bebauter Gebiete besondere Gefahren ausgehen. Ein Benutzungsrecht dieser Radwege ist mit dem Zusatzzeichen „Radfahrer frei“ zu kennzeichnen (VwV-StVO zu § 2, Absatz 4, Satz 3 und 4).

\subsection{Verkehrsrechtliche Regelungen außerhalb Deutschlands}

\subsubsection{Schweiz}

Die Verkehrsregeln des Schweizer „Strassenverkehrsgesetzes“ gelten für „die Führer von Motorfahrzeugen und die Radfahrer auf allen dem öffentlichen Verkehr dienenden Strassen“ (vgl. Art. 1, SVG 2015). Gemäß Artikel 43, Absatz 2 ist der Radweg den Radfahrern vorbehalten und nach Artikel 46, Absatz 1 müssen die Radfahrer die Radwege und -streifen benutzen [SVG 2015].

Entsprechend Artikel 33, Absatz 1 der „Signalisationsverordnung“ verpflichtet das Signal „Radweg“ (Signal 2.60) die Führer von einspurigen Fahrrädern, den für sie gekennzeichneten Weg zu benutzen. Nach Artikel 33, Absatz 4 gilt dies auch für „Rad- und Fusswege mit getrennten Verkehrsflächen" (Signal 2.63) und "Gemeinsame Rad- und Fusswege" (Signal 2.63.1) [SVV 2015]. Die genannten Signale (vgl. Anhang A) entsprechen sinngemäß den deutschen Verkehrszeichen nach Abbildung 3. Nach Artikel 65, Absatz 8 darf insbesondere zur Schulwegsicherung auf relativ stark befahrenen Straßen am Beginn eines schwach begangenen Gehweges das Signal „Fussweg“ (2.61) mit der Zusatztafel „Radfahrer gestattet“ angebracht werden. In diesem Fall gelten die Bestimmungen über eine gemeinsame Benutzung gemäß o.g. Artikel 33 Absatz 4 [SVV 2015]. Gemäß Artikel 74, Absatz 7 kann auf Radwegen und Radstreifen (nach Artikel 74, Absatz 5 durch eine unterbrochene oder ununterbrochene, gelbe Linie vom Kraftfahrzeugverkehr abgegrenzt) das Symbol eines Fahrrades in gelber Farbe markiert werden [SVV 2015]. Nicht benutzungspflichtige Radwege existieren in den aktuell geltenden Schweizer Gesetzen und Verordnungen nicht. 


\subsection{2 Österreich}

In der österreichischen StVO sind entsprechend § 2 Radwege für den Verkehr mit Fahrrädern bestimmte und auch als solche gekennzeichnete Wege. Radfahrstreifen dagegen sind ein für den Fahrradverkehr bestimmter und besonders gekennzeichneter Teil der Fahrbahn, welcher im Verlauf durch wiederholte Markierung mit Fahrradsymbolen hervorzuheben und dessen Ende durch die Schriftzeichenmarkierung „Ende“ anzuzeigen ist [StVO 2015].

Weiterhin regelt $\S 68$ das Verhalten der Radfahrer. Nach Absatz 1 ist auf Straßen mit einer Radfahranlage mit einspurigen Fahrrädern ohne Anhänger die Radfahranlage zu benutzen, wenn das Befahren der Radfahranlage in der vom Radfahrer beabsichtigten Fahrtrichtung erlaubt ist. Nach § 8a, Absatz 1 dürfen Radfahranlagen hier auch in beiden Fahrtrichtungen befahren werden, sofern nicht Bodenmarkierungen andere Vorgaben anzeigen (dies gilt jedoch nicht für Radfahrstreifen). Fahrräder mit Anhängern (weniger als $80 \mathrm{~cm}$ Breite), Fahrräder mit Anhängern zur Personenbeförderung, mehrspurige Fahrräder (weniger als $80 \mathrm{~cm}$ Breite) sowie Rennfahrräder zu Trainingszwecken dürfen nach $\S 68$, Absatz 1 die Radfahranlagen benutzen. Alle anderen Fahräder müssen den für den übrigen Verkehr bestimmten Teil der Fahrbahn benutzen [StVO 2015].

Die entsprechenden Radverkehrsanlagen werden durch die Vorschriftszeichen nach $\S 52$, Absatz b "Gebotszeichen“ angezeigt und erlauben den Fahrern einspuriger Fahrräder ausschließlich die Benutzung des ausgewiesenen Radweges (vgl. hierzu Anhang A). Auch hier ist das Grundkonzept der Verkehrszeichen mit den deutschen Verkehrszeichen nach Abbildung 3 vergleichbar.

Nach § 68, Absatz 1a dürfen aber auch - „wenn es der Leichtigkeit und Flüssigkeit des Fahrradverkehrs dient und aus Gründen der Leichtigkeit und Flüssigkeit des übrigen Verkehrs sowie der Verkehrssicherheit keine Bedenken dagegen bestehen" - abweichend von $\S 68$, Absatz 1 von Radfahrern bestimmte Radwege oder Geh- und Radwege benutzt werden ohne das eine Benutzungspflicht besteht [StVO 2015]. Diese „Radwege ohne Benützungspflicht“ werden durch eckige Hinweiszeichen nach § 53, Absatz 1 angezeigt, welche auf verkehrswichtige Umstände hinweisen sollen (vgl. Abbildung 4). Ein entsprechendes Ende der nicht benutzungspflichtigen Radverkehrsanlagen wird durch die gleichen Verkehrszeichen mit einem roten Querbalken angezeigt.

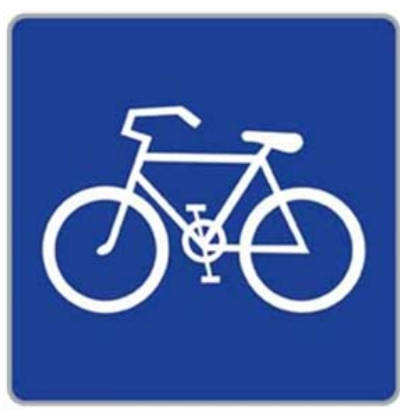

Z 27 „Radweg ohne Benützungspflicht“

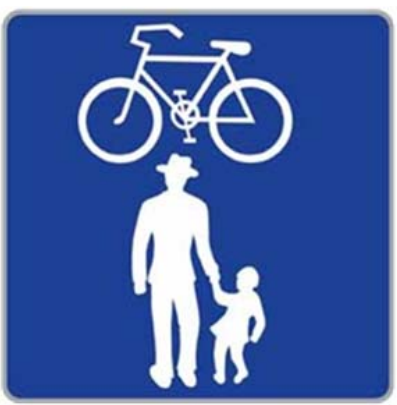

Z 28a „gemeinsamer Geh- und Radweg ohne Benützungspflicht"

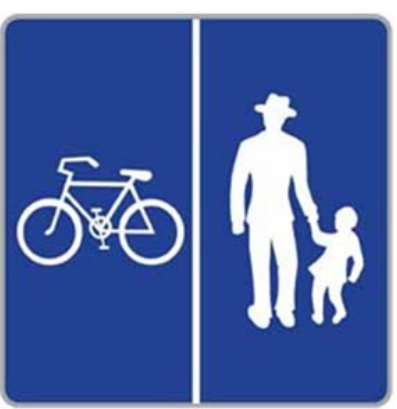

Z 28b „getrennter Geh- und Radweg ohne Benützungspflicht"

Abbildung 4: Verkehrszeichen für Radwege ohne Benützungspflicht in Österreich [Bildquelle: www.kommunalbedarf.at]

Demnach existieren in Österreich sowohl benutzungspflichtige als auch nicht benutzungspflichtige Radwege. Unterschied zu den verkehrsrechtlichen Vorgaben in Deutschland ist jedoch die direkte Kennzeichnung der nicht benutzungspflichtigen Radwege mit gesonderten (eckigen) Verkehrszeichen. Radwege mit Benutzungspflicht entsprechen dagegen den verkehrsrechtlichen Regelungen in Deutschland und werden durch sinngemäß ähnliche, runde Verkehrszeichen angezeigt. 


\subsubsection{Italien}

In Italien sind die Verkehrsregeln im „Nuovo codice della Strada, DL 30.04.1992, $n^{\circ} 285^{\prime}$ [DL 1992] festgehalten. Artikel 182, Absatz 9 beinhaltet hierbei die Regelungen zum Radverkehr und dass durch Radfahrer die vorhandenen Radwege zu benutzen sind. Weiterhin enthalten die „Regolamento di esecuzione e di attuazione del nuovo codice della strada, DPR 16.12.1992, $n^{\circ} 495^{\prime \prime}$ [DPR 1992] die Vorgaben zur Kennzeichnung der Radwege. Dementsprechend müssen alle Radwege am Beginn mit einem Verkehrszeichen ausgestattet sein, welches nach Unterbrechungen oder Knotenpunkten zu wiederholen ist (Artikel 122, Absatz 9b). Dabei müssen die nutzungsberechtigten Verkehrsmittel als Symbol im entsprechenden Verkehrszeichen erkennbar sein. Für diese Verkehrsmittel ist die Nutzung vorgeschrieben, für alle anderen verboten. Die zugehörigen Verkehrszeichen nach Anhang A entsprechen ebenfalls sinngemäß den deutschen Verkehrszeichen nach Abbildung 3. Das Ende eines Radweges wird durch eben diese Verkehrszeichen mit einem roten Querbalken verdeutlicht [DPR 1992]. Radwege die dagegen nicht durch vertikale bauliche Elemente vom Kraftfahrzeugverkehr getrennt werden (Radfahrstreifen), müssen durch zwei durchgehende Linien (12 cm breite weiße Linie auf Seiten der Kraftfahrzeuge und $30 \mathrm{~cm}$ breite gelbe Linie auf Seiten des Radfahrstreifens) markiert sein (Artikel 140, Absatz 7, DPR 16.12.1992, n495). Die entsprechende Benutzungspflicht kann neben Verkehrszeichen Figura II 90 (vgl. Anhang A) auch als Markierung im Radfahrstreifen wiederholt werden (Artikel 148, Absatz 11, DPR 16.12.1992, $n^{\circ} 495$ ) [DPR 1992]. Zusammenfassend müssen alle Radwege in Italien durch entsprechende Verkehrszeichen gekennzeichnet werden und sind entsprechend auch benutzungspflichtig. Nicht benutzungspflichtige Radwege existieren in Italien dagegen nicht.

\subsubsection{Niederlande}

Die niederländischen Verkehrsregeln sind in der aktuell gültigen Fassung von 2015 des „Reglement verkeersregels en verkeerstekens 1990“ festgehalten [RVV 2015]. In Kapitel 2 der RVV sind in § 1 die Verkehrsregeln an Straßen festgelegt, Artikel 5 enthält hier die Regelungen für den Radverkehr. Gemäß Artikel 5, Satz 1 müssen Radfahrer benutzungspflichtige Radwege oder benutzungspflichtige gemeinsame Rad-/Mopedwege benutzen und dürfen nach Artikel 5, Satz 2 nur die Fahrbahn nutzen, wenn derartige benutzungspflichtige Radwege nicht vorhanden sind. Nach Anlage 1 der RVV werden in Kapitel G „Verkehrsregeln“ diese Radwege durch die Zeichen G 11 und G 12a gekennzeichnet (vgl. Anhang A dieses Berichtes). Somit sind auch hier die verkehrsrechtlichen Regelungen bezüglich benutzungspflichtiger Radwege ähnlich der deutschen Straßenverkehrsordnung mit dem Unterschied, dass keine gemeinsamen Geh- und Radwege existieren, jedoch gemeinsame Rad- und Mopedwege angeordnet werden können. Zudem dürfen Radfahrer nach Artikel 5, Satz 3 vorhandene nicht benutzungspflichtige Radwege benutzen. Für Mofas mit Verbrennungsmotor gilt dies nur mit ausgeschaltetem Motor. Vergleichbar mit den österreichischen Regelungen werden nicht benutzungspflichtige Radwege durch ein gesondertes eckiges Verkehrszeichen (Zeichen G 13 nach Anlage 1, Kapitel G, RVV 2015) angezeigt (siehe Abbildung 5).

\section{fietspad}

Abbildung 5: Kennzeichnung nicht benutzungspflichtiger Radwege in den Niederlanden [Bildquelle: Verkeersborden en Verkeersregels in Nederland, Ministerie van Infrastructuur en Milieu, 2014]

Fahrräder mit mehr als zwei Rädern (mehrspurige Fahrräder) sowie Fahrräder mit Anhänger, welche jeweils einschließlich der Ladung breiter als 0,75 $\mathrm{m}$ sind, dürfen dagegen grundsätzlich die 
Fahrbahn nutzen (vgl. Kapitel 2, § 1, Artikel 5, Satz 3, RVV 2015). Endet ein benutzungspflichtiger oder nicht benutzungspflichtiger Radweg, so wird dies durch die entsprechenden Verkehrszeichen mit einem roten Querbalken verdeutlicht (vgl. RVV 2015).

\subsubsection{Frankreich}

In Frankreich sind die Regelungen für Verkehrszeichen und für das entsprechende Verkehrsverhalten in den „Instruction Interministérielle sur la signalisation routière“ geregelt. Teil 4 enthält hierbei die Vorschriftszeichen [IISR 2013a] und Teil 5 die Hinweisschilder [IISR 2013b].

Die Benutzungspflicht von Radwegen anordnenden Verkehrszeichen sowie die entsprechenden Regelungen sind in Teil 4, Artikel 66 festgelegt. Zeichen B 22a zeigt dementsprechend an, dass Radfahrer die entsprechenden Radwege benutzen müssen und alle anderen Fahrzeuge diese Bereiche nicht benutzen dürfen. Im Regelfall dürfen auf diesen Radwegen ebenfalls Kleinkrafträder fahren, was dann durch ein entsprechendes Zusatzzeichen (M4d2) zu Zeichen B 22a anzuzeigen ist. Weiterhin können die Radwege an deren Beginn mit einer Richtungsweisung (Einrichtungsradweg bzw. Zweirichtungsradweg) verbunden werden. An Knotenpunkten sind die Radwegzufahrten in allen Knotenpunktarmen entsprechend anzuzeigen [IISR 2013a]. Die entsprechenden Zeichen sind in Anhang A dargestellt. Die grundlegende Regelung für die Beschilderung von benutzungspflichtigen Radwegen entspricht somit den Vorgaben der deutschen StVO. Zeichen für benutzungspflichtige gemeinsame oder getrennte Geh- und Radwege existieren in Frankreich nicht.

Wie in der österreichischen Straßenverkehrsordnung existieren auch in Frankreich eckige Verkehrszeichen, um nicht benutzungspflichtige Radwege anzuzeigen. Nach Teil 5, Artikel 75-3 der „Instruction Interministérielle sur la signalisation routière“ ist die Anzeige nicht benutzungspflichtiger Radwege seitens der Verwaltungen verpflichtend, um auf die für Radfahrer vorgesehenen Wege hinzuweisen [IISR 2013b]. Dies geschieht entweder durch Zeichen C 113 (IISR 2013b) oder Radfahrerpiktogramme (vgl. Artikel 118-1, IISR 2011). Die Beschilderungen können zur Freigabe der nicht benutzungspflichtigen Radwege für Kleinkrafträder durch Zusatzzeichen ergänzt werden. Das Ende benutzungspflichtiger und nicht benutzungspflichtiger Radwege wird durch die entsprechenden gleichen Verkehrszeichen mit einem roten Querbalken angezeigt [IISR 2013b].

\subsubsection{Fazit}

Nach der Analyse nationaler und internationaler Gesetzgebungen ergeben sich folgende Schlussfolgerungen. Generell existieren 3 Arten zur Führung des Radverkehrs in Bezug auf Radwege:

- Führungen auf der Fahrbahn (keine Radwege vorhanden)

- auf benutzungspflichtigen Radwegen und

- auf nicht benutzungspflichtigen Radwegen.

Bei den untersuchten Staaten zeigt sich in der Schweiz und in Italien, dass entweder die Fahrbahnführung des Radverkehrs oder die Führung auf benutzungspflichtigen Radwegen in den Verkehrsregelungen enthalten sind und nicht benutzungspflichtige Radwege somit in diesen Staaten nicht existieren. Wenn Radwege vorhanden sind, dann sind diese auch grundlegend zu benutzen (Benutzungspflicht). In Deutschland, Österreich, den Niederlanden und in Frankreich gibt es dagegen auch nicht benutzungspflichtige Radwege neben den beiden anderen Führungsformen. Der Radfahrer verfügt hier über ein Nutzungsrecht des vorhandenen Radweges, kann aber selbst entscheiden, ob er auf der Fahrbahn oder dem angrenzenden nicht benutzungspflichtigen Radweg fahren möchte. Eine Verbindlichkeit der Radwegnutzung besteht hier jedoch nicht. 
In Hinblick auf die Beschilderung bzw. Kennzeichnung von nicht benutzungspflichtigen Radwegen gibt es weiterhin zwei verschiedene Grundkonzepte. In Deutschland werden die nicht benutzungspflichtigen Radverkehrsanlagen gemäß StVO und VwV-StVO nicht gesondert ausgeschildert. Ist hier ein straßenbegleitender Radweg vorhanden, welcher nicht durch die entsprechenden Beschilderungen benutzungspflichtig ist, kann der Radfahrer diesen Radweg oder auch die Fahrbahn nutzen. Die Erkennbarkeit dieser nicht benutzungspflichtigen Radwege als solche muss dabei gewährleistet sein (z.B. durch farbliche, materialtechnische Hervorhebungen oder Piktogramme), ein gesondertes Verkehrszeichen ist nicht vorgesehen. Ausnahme ist hier lediglich die Anordnung Gehweg Radfahrer frei, bei der ein konkreter Hinweis auf die mögliche Radverkehrsführung im Seitenraum gegeben wird. Hier stehen dem Radfahrer jedoch keine eigenen Verkehrsflächen zur Verfügung, er muss stattdessen auf den Fußgängerverkehr besondere Rücksicht nehmen.

Neben der fehlenden Möglichkeit der Beschilderung in Deutschland, welche auch durch die sogenannte „Schilderwaldnovelle“ der StVO von 2009 mit bedingt ist, existieren dagegen in Österreich, den Niederlanden und in Frankreich miteinander vergleichbare Konzepte zur Beschilderung nicht benutzungspflichtiger Radwege. In Österreich und Frankreich gibt es - neben den mit den deutschen Regelungen vergleichbaren, runden Verkehrszeichen für benutzungspflichtige Radwege die gleichen Verkehrszeichen für nicht benutzungspflichtige Radwege, jedoch in eckiger Form. In den Niederlanden werden die nicht benutzungspflichtigen Radwege ebenfalls durch ein eckiges Zeichen mit der Aufschrift „fietspad“ gekennzeichnet. Enden die entsprechenden Radverkehrsanlagen, so werden die gleichen Verkehrszeichen mit einem roten Querbalken angeordnet. Konkretere Bedingungen (z.B. Radwegbreite) für die Anordnung benutzungspflichtiger oder nicht benutzungspflichtiger Radwege sind in den internationalen Gesetzen und Verordnungen nicht enthalten.

\subsection{Empfehlungen für Radwege in den deutschen Planungsregelwerken}

In Deutschland sind die wesentlichen Grundlagen zur Planung von Radverkehrsanlagen in den Richtlinien für die Anlage von Stadtstraßen [RASt 2006] und den Empfehlungen für Radverkehrsanlagen [ERA 2010] enthalten. Das vorliegende Kapitel fasst die entsprechenden Empfehlungen zusammen. Der Fokus der Ausarbeitungen liegt hierbei auf den Führungsformen des Radverkehrs und den entsprechenden Einsatzempfehlungen.

Zur Auswahl bestimmter Straßenquerschnitte für die „typischen Entwurfssituationen“ nach RASt [2006] werden bezüglich des Radverkehrs die folgenden Abwägungsgrundsätze angegeben. Bei Kraftfahrzeugstärken unter $400 \mathrm{Kfz} / \mathrm{h}$ kann der Radverkehr grundlegend auf der Fahrbahn geführt werden, zwischen 400 und $1.000 \mathrm{Kfz} / \mathrm{h}$ werden Schutzstreifen und bei mehr als $1.000 \mathrm{Kfz} / \mathrm{h}$ Radfahrstreifen oder Radwege empfohlen. Bei diesen Festlegungen sind aber auch die verfügbaren Straßenraumbreiten zu berücksichtigen [RASt 2006]. Die Empfehlungen für Radverkehrsanlagen dienen zur Vertiefung und Ergänzung der maßgebenden Planungsrichtlinien (u.a. RASt) und enthalten hier deutlich detailliertere Anforderungen an die Radverkehrsanlagen. Für den Entwurf und die Auswahl von Radverkehrsanlagen an Straßen gelten die folgenden Anforderungen:

- objektive Sicherheit (z.B. geringes Unfallrisiko, hohe Akzeptanz, gute Sichtverhältnisse, geringes Sturz- und Gefährdungsrisiko)

- $\quad$ subjektive Sicherheit (z.B. Vermeidung gefährlicher, überfordernder Situationen)

- Qualitätskriterien (z.B. Ermöglichen von Überholungen zur Berücksichtigung unterschiedlicher Radverkehrsgeschwindigkeiten, Minimierung des Kraftaufwandes durch Oberflächenbeschaffenheit sowie minimale Umwege/Steigungen/Halte und Minimierung von Zeitverluste durch optimierte Knotenpunktgestaltung und -signalisierung) [ERA 2010] 
Zur Auswahl bestimmter Radverkehrsanlagen enthalten die ERA 2010 eine Verfahrensweise zur Entscheidungsfindung und Abwägung für verschiedene straßenräumliche Situationen, welche für den Neu-, Um- und Rückbau verwendet werden kann. Die „typischen Entwurfssituationen“ der RASt dienen dagegen für komplette Straßenumgestaltungen. Das vorgegebene Verfahren beinhaltet eine Vorauswahl von Führungsformen, eine Prüfung der Realisierbarkeit möglicher Führungsformen sowie abschließend ein Vergleichsverfahren für potentiell mehrere geeignete Varianten, welche in den nachfolgenden Kapiteln vorgestellt werden [ERA 2010].

\subsubsection{Vorauswahl von Führungsformen des Radverkehrs}

Das Verfahren zur Vorauswahl einer geeigneten Führungsform des Radverkehrs basiert im Wesentlichen auf den Verkehrsstärken (Prognosebelastung der werktäglichen Spitzenstunde) und den Geschwindigkeiten des Kraftfahrzeugverkehrs (zulässige Höchstgeschwindigkeit $\mathrm{V}_{\text {zul }}$ bzw. in Ausnahmefällen die Quantilgeschwindigkeit $V_{85}$ ). Hierfür definiert die ERA [2010] die in Abbildung 6 dargestellten vier Belastungsbereiche.

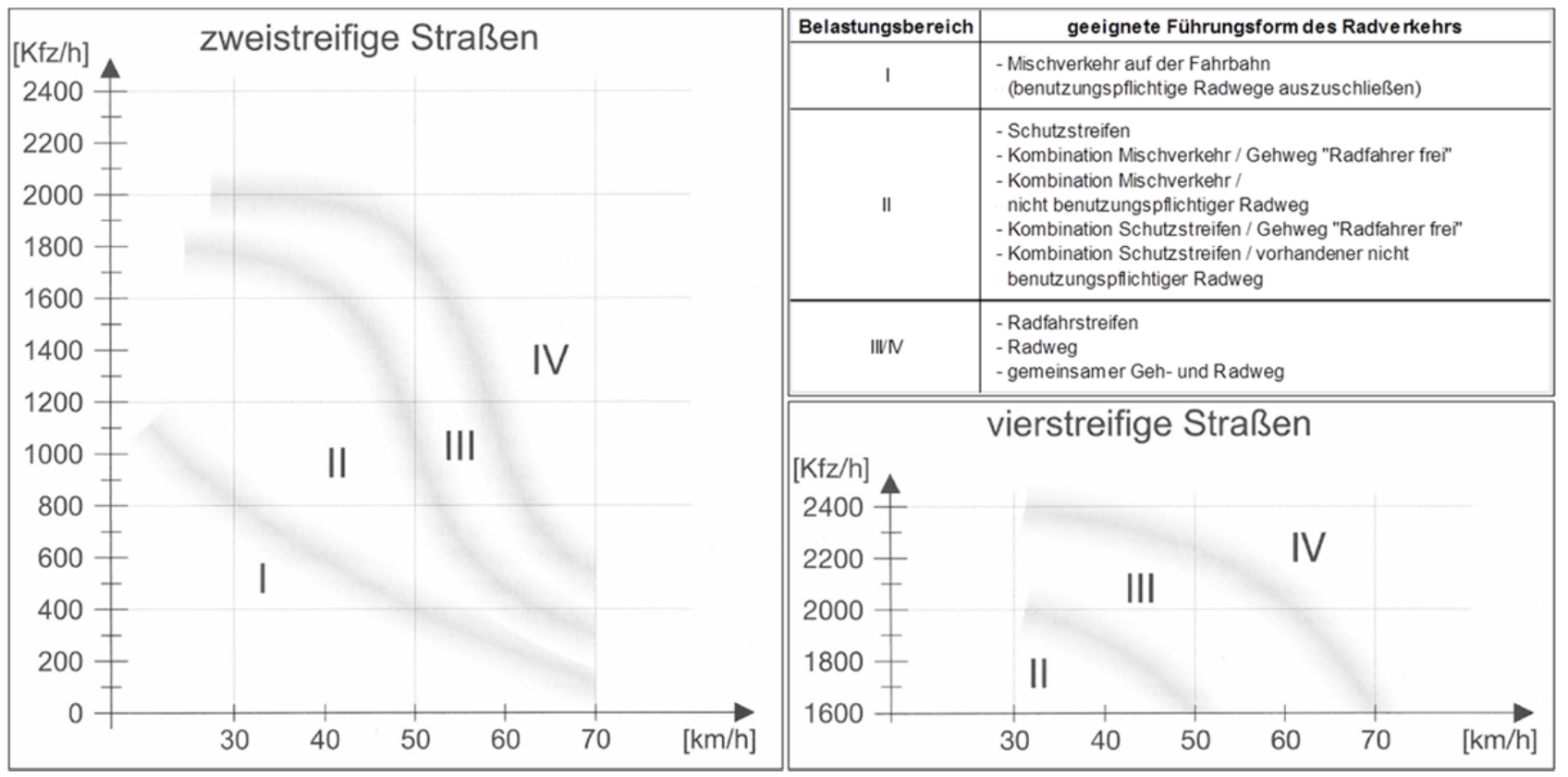

Abbildung 6: Belastungsbereiche und zugeordnete geeignete Radverkehrsführungen an zwei- und vierstreifigen Stadtstraßen nach ERA [2010]

Bei den Belastungsbereichen I und II ist die Fahrbahnführung des Radverkehrs grundsätzlich vertretbar, wobei bei Vorliegen von Belastungsbereich II zusätzliche Maßnahmen, wie beispielsweise Gehweg Radfahrer frei bzw. nicht benutzungspflichtige Radwege oder Schutzstreifen, umgesetzt werden sollten. Eine Trennung des Radverkehrs vom Kraftfahrzeugverkehr kann bei Belastungsbereich III erforderlich werden. Hier kann der Radverkehr aber bei günstigen Randbedingungen noch im Mischverkehr geführt werden. Aus Sicherheitsgründen ist bei Belastungsbereich IV eine Trennung von Rad- und Kraftfahrzeugverkehr generell geboten [ERA 2010].

Die entsprechenden Festlegungen können somit zur Auswahl einer notwendigen Radverkehrsführung im Straßenseitenraum aufgrund einer sonst vorhandenen potentiellen Gefährdung herangezogen werden. Die Belastungsbereiche dienen hierbei als Orientierung, wann aufgrund der verkehrlichen Rahmenbedingungen (Verkehrsstärke und Geschwindigkeit) eine Gefährdung bzw. ein erhöhtes Verkehrssicherheitsrisiko besteht und wann eine Führung des Radverkehrs im Mischverkehr vermieden werden sollte [ERA 2010]. Hierbei handelt es sich jedoch nur um eine Grundgefährdung des Radverkehrs, nicht aber um eine besondere Gefahrenlage nach StVO [Alrutz 2015]. 


\subsubsection{Realisierbarkeit von Radverkehrsführungen}

Die anschließende Überprüfung der Realisierbarkeit einer ausgewählten Führungsform im Radverkehr findet - neben den bereits betrachteten Verkehrsstärken und Geschwindigkeiten - anhand des Straßenraumes bzw. der zur Verfügung stehenden Breiten statt. Die grundlegenden Anforderungen an Verkehrsräume von Radfahrern nach RASt [2006] sind in Abbildung 7 dargestellt.

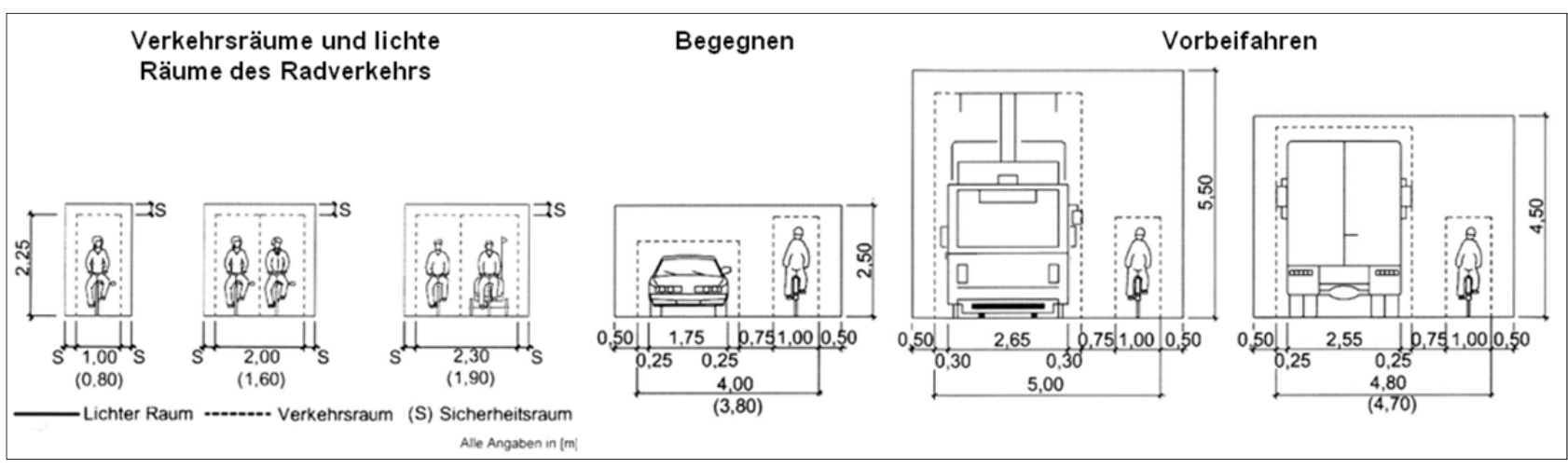

Abbildung 7: Verkehrsräume und lichte Räume des Radverkehrs nach RASt [2006]

Die Mischverkehrsführung ist bei Fahrbahnbreiten zwischen 6,00 m und 7,00 m und Verkehrsstärken über $400 \mathrm{Kfz} / \mathrm{h}$ als problematisch anzusehen [ERA 2010]. Hier sind Überholungen bei Gegenverkehr nur ohne Einhaltung des notwendigen Sicherheitsabstandes möglich und gelten als kritisch. Eine Führung im Mischverkehr eignet sich hier nur bei geringen Verkehrsstärken und Geschwindigkeiten [RASt 2006].

Bei Fahrbahnbreiten unter 6,00 m ist eine Mischverkehrsführung bis zu $700 \mathrm{Kfz/h}$ verträglich, über 7,00 m kann im Kfz-Kfz Begegnungsfall der Radverkehr mit ausreichendem Sicherheitsabstand überholt werden, ab 7,50 m soll aber der Einsatz von Schutzstreifen geprüft werden. Bei höheren als den eben genannten Verkehrsstärken sind bei den entsprechenden Fahrbahnbreiten andere Führungsformen anzustreben [ERA 2010]. In den RASt [2006] wird eine mögliche Mischverkehrsführung bei Fahrbahnbreiten bis $6,00 \mathrm{~m}$ und Verkehrsstärken bis $500 \mathrm{Kfz} / \mathrm{h}$ sowie bei Fahrbahnbreiten über 7,00 m und Verkehrsstärken von $800 \mathrm{Kfz} / \mathrm{h}$ bis $1.000 \mathrm{Kfz} / \mathrm{h}$ sowie einem Schwerverkehrsanteil unter $6 \%$ grundsätzlich für möglich gehalten. Bei vierstreifigen Stadtstraßen ist eine Mischverkehrsführung ab Verkehrsstärken von $2.200 \mathrm{Kfz} / \mathrm{h}$ nicht mehr verträglich [ERA 2010].

Sollen dem Radverkehr bei mindestens 7,00 m breiten Fahrbahnen (im Regelfall 7,50 m) und eigentlich verträglicher Mischverkehrsführung aus Sicherheitsgründen eigene Flächen auf der Fahrbahn zugewiesen werden, so bieten sich 1,50 m breite Schutzstreifen an, wenn die verfügbare Breite für Radfahrstreifen nicht ausreicht [RASt 2006]. Die Schutzstreifen auf der Fahrbahn dürfen beim Lkw-Lkw Begegnungsfall bei Bedarf überfahren werden. Übersteigt die Anzahl der Schwerverkehrsfahrzeuge hier jedoch $1.000 \mathrm{Kfz} / 24 \mathrm{~h}$, so ist von Schutzstreifen abzuraten [ERA 2010].

Werden aufgrund höherer Verkehrsstärken oder Geschwindigkeiten Radfahrstreifen notwendig, so sollten diese einschließlich Breitstrich zur Abgrenzung von der Fahrbahn mindestens 1,85 m breit sein. Der verbleibende Teil der Fahrbahn sollte dabei noch eine Breite von mindestens $5,50 \mathrm{~m}$, im Regelfall sogar 6,50 m aufweisen. Somit werden Fahrbahnbreiten von insgesamt mindestens 9,20 m, im Regelfall 10,20 m erforderlich. Ein Radfahrstreifen ist mit Zeichen 237 StVO als benutzungspflichtige Radverkehrsanlage zu kennzeichnen [ERA 2010].

Bei erforderlichen baulich angelegten Radwegen müssen im Straßenseitenraum 2,00 m, im Minimalfall 1,60 m eingeplant werden. Im Verlauf von Radverkehrshauptrouten, bei höheren Radverkehrsbelastungen, häufigen Belastungsspitzen, intensiver Seitenraumnutzung oder starkem Gefäl- 
le können breitere Radwege erforderlich werden [ERA 2010]. Da unterhalb von 1,60 m Breite eines Radweges keine gegenseitigen Überholungen mehr möglich sind, sollte bei derartigen Breiten von einer Benutzungspflicht abgesehen werden. Grundlegend kann auch die Nichtanlage einer gesonderten Radverkehrsanlage geschwindigkeitsdämpfende Maßnahmen im Kraftfahrzeugstrom erforderlich machen. Weiterhin erfordert das Regelmaß von 6,50 m für Fahrbahnbreiten an Hauptverkehrsstraßen nach RASt [2006] benutzungspflichtige Radverkehrsanlagen.

Außerdem sind bei Planungen entsprechend der Aufenthaltsfunktion nach RASt [2006] die verbleibenden Breiten der Gehwege zu berücksichtigen. Zwischen Geh- und Radwegen sind zudem Sicherheitstrennstreifen vorzusehen. Die Sicherheitstrennstreifen sind höhengleiche Begrenzungsstreifen mit taktilen und optischen Unterschieden und einer Breite von 0,30 m [ERA 2010]. Zu anderen Querschnittelementen gelten die nachfolgenden Breiten von Sicherheitstrennstreifen:

- $\quad$ 0,75 m zum Fahrbahnrand mit Einbauten im Sicherheitstrennstreifen bzw. KfzGeschwindigkeiten von über $50 \mathrm{~km} / \mathrm{h}$

- $\quad$ 0,50 m zum Fahrbahnrand in sonstigen Fällen

- $\quad 0,75 \mathrm{~m}$ zu in Längsrichtung parkenden Fahrzeugen

- $\quad$ 1,10 m zu parkenden Fahrzeugen in Schräg- und Senkrechtaufstellung [ERA 2010]

Bei nicht vertretbarer Fahrbahnführung und weiterhin nicht realisierbaren Radwegen oder Radfahrstreifen sowie verträglichen (geringen) Fußgänger- und Radverkehrsbelastungen können gemeinsame Geh- und Radwege angelegt werden. Im Hinblick auf schnelle Radfahrer und deren möglicher Fahrbahnführung sollte aber die Regelung Gehweg Radfahrer frei favorisiert werden. Bei z.B. intensiver Geschäftsnutzung, vielen schutzbedürftigen Fußgängern, in Radhauptverbindungen, starkem Gefälle (über $3 \%$ ) oder einer Vielzahl von Knotenpunkten oder stark frequentierten Haltestellen ist eine gemeinsame Führungsform jedoch auszuschließen [EFA 2002]. Grundlegend kommen gemeinsame Geh- und Radwege nur bei geringer Netz- und Aufenthaltsfunktion im Rad- und Fußgängerverkehr in Betracht [ERA 2010].

Die Nutzung von Radwegen entgegen der eigentlichen Fahrtrichtung ist innerorts eine häufige Unfallursache. Baulich angelegte Zweirichtungsradwege dürfen daher nur nach sorgfältiger Prüfung angelegt werden [ERA 2010] und gelten für diese Untersuchung eher als Sonderfall.

\subsubsection{Vergleich und Bewertung verschiedener Führungsformen}

Kommen für eine Stadtstraße nach der Vorauswahl und der Überprüfung der Realisierbarkeit mehrere unterschiedliche Führungsformen in Frage, ist abschließend nach ERA [2010] für alle potentiellen Führungsformen eine Bewertung und ein Vergleich vorzunehmen. Als Bewertungskriterien werden hierbei die folgenden Parameter herangezogen:

- Kraftfahrzeugverkehrsstärke

- Schwerverkehrsstärke

- Flächenverfügbarkeit

- Parken

- Knotenpunkte und Grundstückszufahrten

- Längsneigung 
Bei erhöhten Kraftfahrzeug- und Schwerverkehrsstärken wird von höheren Gefährdungen des Radverkehrs auf der Fahrbahn ausgegangen und eine Führung im Seitenraum bevorzugt. Die Flächenverfügbarkeit bewertet die Nutzbarkeit der Führungsform bezüglich deren Breite. Weiterhin müssen die von Parkvorgängen ausgehenden Gefährdungen für Radverkehrsführungen auf der Fahrbahn bewertet werden. Hierzu zählt neben dem Parken im Seitenraum auch das Liefern und Halten in zweiter Reihe. Ist hier insgesamt eine erhöhte Frequenz feststellbar, so sind Führungen im Seitenraum zu bevorzugen. Gegen eine Radverkehrsführung im Seitenraum spricht jedoch eine erhöhte Anzahl von Knotenpunkten und Einmündungen im Streckenzug und hier ebenfalls eine erhöhte Anzahl von ein- und abbiegenden Fahrzeugen [ERA 2010].

Bei größeren Längsneigungen über $3 \%$ sollte der höhere Breitenbedarf von Radfahrern berücksichtigt werden. Bergab erzeugen die höheren Geschwindigkeiten der Radfahrer in Kurven einen größeren Lichtraumbedarf, bergauf ergibt sich durch instabilere Fahrweise oder das Schieben der Fahrräder ebenfalls ein größerer Flächenbedarf. Starke, längere Steigungen erfordern eher eine Führung im Seitenraum, die entsprechenden Gefälle dagegen vorzugsweise eine Fahrbahnführung. Bei den Bewertungen kann also auch eine asymmetrische Querschnittsaufteilung mit unterschiedlichen Führungsformen bergauf und bergab erforderlich werden [ERA 2010].

Insgesamt ist oftmals eine qualitative Prüfung der oben genannten Bewertungskriterien ausreichend, für schwierig zu beurteilende Situationen steht in der Anlage der ERA noch ein Bewertungsverfahren mit einem Punktesystem zur Verfügung. Die Punkte ergeben sich hierbei aus den oben erläuterten Kriterien und die Führungsform mit mindestens 10 Punkten oder der insgesamt höchsten Punktzahl kann abschließend als besonders geeignet angesehen werden [ERA 2010].

Nachfolgend wird die Bewertung für Radwege im Seitenraum sowie eine Führung auf der Fahrbahn kurz beschrieben, welche für die im vorliegenden Projekt zu betrachtende Fragestellung der Benutzungspflicht von Radwegen am wesentlichsten ist. Zur Bewertung der Kraftfahrzeugverkehrsstärke werden hier erneut die Belastungsbereiche herangezogen. Eine gleichwertige Punktezahl ergibt sich für die Führung im Seitenraum und der Fahrbahnführung bei den Belastungsbereichen II und III. Bezüglich des Schwerverkehrs führen weniger als 300 Schwerverkehrsfahrzeuge pro Tag zur gleichberechtigten Anordnung von Seitenraum- und Fahrbahnführungen. Gleiches trifft für geringen Parkdruck, weniger als 4 Einfahrten je Kilometer und eine Anzahl von abbiegenden Fahrzeugen von weniger als 100 pro Tag zu [ERA 2010]. Entsprechend der oben beschriebenen Zusammenhänge kann sich die beschriebene gleichwertige Punktvergabe bei Überschreitung der genannten Grenzwerte zugunsten einer bestimmten Führungsform verändern und somit zu einer Bevorzugung von Radwegen im Seitenraum oder eben der Fahrbahnführung führen. Bei dem Bewertungsverfahren ist jedoch auch zu beachten, dass erst die Überlagerung und Abwägung aller Kriterien zu einer Entscheidung führen kann. Somit ist bei Nichterfüllung eines Kriteriums nicht zwangsläufig eine bestimmte Führungsform auszuschließen. Grundsätzlich sollten dann aber auch die abweichenden Kriterien gesonderte Beachtung finden. Bezüglich der Benutzungspflicht von Radwegen können die genannten Kriterien ebenfalls zur Bewertung herangezogen werden.

\subsubsection{Weitere Einflussgrößen auf die Führungsformen des Radverkehrs}

Neben den im Bewertungsverfahren enthaltenen Kriterien existieren aber noch weitere zu beachtende Sonderfälle, welche Einfluss auf die Auswahl einer bestimmten Führungsform bzw. die Frage nach der Benutzungspflicht von Radwegen haben können.

Ist beispielsweise bei Fahrbahnen mit Straßenbahngleisen eine Radverkehrsführung im Seitenraum, welche Konflikte mit den Straßenbahnen weitestgehend ausschließt, nicht möglich, so gelten für die Fahrbahnführung aufgrund der Rillenschienen und Sturzgefahr besondere Randbedin- 
gungen. Bei der Führung des Radverkehrs auf der Fahrbahn soll hier im Regelfall eine Führung rechts der Straßenbahngleise gewählt werden. Sollen hier Überholmöglichkeiten der Radfahrer untereinander zur Verfügung gestellt werden, so sind zwischen Bord und Verkehrsraum der Straßenbahn mindestens 1,60 $\mathrm{m}$ vorzusehen. Wird bei geringem Radverkehrsaufkommen auf Überholmöglichkeiten verzichtet, genügen mindestens 1,30 m. In Ausnahmefällen können auf kurzen Abschnitten aufgrund besonderer planerischer Zwangspunkte auch Sonderlösungen für den Radverkehr notwendig werden. Zu diesen zählt die Führung des Radverkehrs im Gleisbereich oder eine Führung rechts der Straßenbahngleise aber teilweise im Verkehrsraum der Straßenbahn (u.a. nur bei maximal $30 \mathrm{~km} / \mathrm{h}$ zulässiger Höchstgeschwindigkeit). Sind diese Grundvoraussetzungen nicht erfüllt, so ist gegebenenfalls eine Führung im Straßenseitenraum zu bevorzugen [ERA 2010].

Weiterhin führen Haltestellen des städtischen Busverkehrs zu Konfliktpunkten mit dem Radverkehr und ebenfalls zu einer Bevorzugung bestimmter Führungsformen. Generell wird hierbei die Fahrbahnführung als konfliktärmer angesehen. Bei der Bewertung der Konfliktlage müssen die Konflikte zwischen Radfahrern und ein-/aussteigenden/wartenden Fahrgästen bei Radwegführung und Konflikte durch wiedereinfädelnde Busse sowie die Konflikte durch hinter dem Bus wartende Radfahrer bei Fahrbahnführung vorrangig beachtet werden. Gleiches gilt im übertragengen Sinne auch für Straßenbahnhaltestellen in Seitenlage. Die ERA [2010] enthalten für die Verkehrsmittel des öffentlichen Personennahverkehrs entsprechende Empfehlungen, welche auch bei der Entscheidung über die Benutzungspflicht von Radwegen herangezogen werden können.

Abschließend ist neben den bisherigen Betrachtungen der Radverkehrsführung an Streckenabschnitten auch die Führung in Knotenpunkten von Interesse. Bei der Entscheidung zur Aufhebung der Radwegebenutzungspflicht kann dies ebenfalls eine wesentliche Rolle spielen. Für den Radverkehr an Knotenpunkten gelten die nachfolgenden Forderungen:

- ausreichend Sichtbeziehungen zu anderen Verkehrsteilnehmern

- zügige und sichere Befahrbarkeit

- Begreifbarkeit der Radverkehrsführung und der Signalisierung für alle Verkehrsteilnehmer

- ausreichend dimensionierte Warteflächen für Radfahrer

- Entschärfung des Konfliktes zwischen geradeaus fahrenden Radfahrern und rechts abbiegenden Kraftfahrzeugen [ERA 2010]

Bei signalgeregelten Knotenpunkten sollten unter anderem auch

- die Freigabezeiten des Radverkehrs nicht erheblich kürzer als die des Kraftfahrzeugverkehrs und

- $\quad$ insgesamt die Wartezeiten möglichst kurz sein [ERA 2010].

Die genannten Kriterien können ebenfalls bei den Entscheidungen für eine neu festzulegende Führungsform im Radverkehr als auch bei bereits vorhandenen Radwegen im Seitenraum und der Frage nach deren weiterer Benutzungspflicht herangezogen werden.

Bei der Signalisierung von Radfahrern an Knotenpunkten gibt es drei wesentliche Grundformen:

1. Gemeinsame Signalisierung mit dem Kraftfahrzeugverkehr

2. Gesonderte Signalisierung des Radverkehrs

3. Gemeinsame Signalisierung mit dem Fußgängerverkehr [RiLSA 2010] 
Diese Signalisierungsformen können bei unterschiedlichen Führungsformen des Radverkehrs angewendet werden, an nicht benutzungspflichtigen Radwegen treten aber Grundform 1 und 3 in den Vordergrund. Für Radfahrer auf der Fahrbahn gilt hier entsprechend Grundform 1, für Radfahrer auf dem nicht benutzungspflichtigen Radweg dagegen Grundform 3. Grundform 2 ist weiterhin für nicht benutzungspflichtige Radwege nur bedingt geeignet und eher ein Ausnahmefall. Um Konflikte zu vermeiden, sollte hier das Radfahrersignal nicht eher Rot zeigen als die Kraftfahrzeugsignalisierung [Stadt Münster 2007].

Bei Aufhebung der Radwegebenutzungspflicht und der möglichen Führung der Radfahrer im Mischverkehr müssen die zwar gemeinsam signalisierten Kraftfahrzeuge und Fahrräder dennoch als getrennte Ströme betrachtet werden. Durch die somit verringerten Räumgeschwindigkeiten der fahrbahngeführten Verkehrsströme ergeben sich auch Veränderungen der Räumzeiten im Vergleich zum vorherigen benutzungspflichtigen Radweg. Grundlegend sind hier die Zwischenzeiten der Signalsteuerung zu überprüfen und bei Bedarf die Signalzeitenpläne anzupassen [RiLSA 2010]. Der Anpassungsbedarf ist jedoch abhängig von der Art und Größe des betrachteten Kontenpunktes. Vorrangig an Knotenpunkten mit langen Räumwegen kann die Erhöhung der Zwischenzeiten dabei auch einen leistungsfähigkeitsmindernden Einfluss bei der Berechnung der Lichtsignalprogramme hervorrufen. Zudem können von Veränderungen der Zwischenzeiten auch vorhandene Koordinierungen mehrerer Lichtsignalanlagen betroffen sein [FGSV 2016].

\subsection{Weitere Empfehlungen und Leitfäden}

Grundlegend haben die in Kapitel 2.3 beschriebenen technischen Regelwerke keinen rechtlich bindenden Charakter für die Benutzungspflicht von Radwegen und dienen nur als Empfehlungen. Auf die jeweils aktuelle ERA wird aber nunmehr durch die VwV-StVO verwiesen und diese Richtlinie wurde im Grundsatzurteil des Bundesverwaltungsgerichtes anerkannt und sollte auch weiterhin für antizipative Sachverständigengutachten zur Gefahrenlage einer Straße herangezogen werden [Klöpfer et al. 2011]. Neben den vielzähligen - über mehrere Gesetze bzw. Verordnungen und Regelwerke hinweg gestreuten - Regelungen im Kontext der Radwegebenutzungspflicht, existieren verschiedene Leitfäden, die bei der Überprüfung der Benutzungspflicht im bestehenden Radwegenetz herangezogen werden können. Verbreitete Leitfäden sind der sogenannte „Mainzer Leitfaden“ [Klöpfer et al. 2011] und der „Leitfaden Radverkehr“ aus Niedersachsen [NLStbV 2013].

Im Mainzer Leitfaden werden sämtliche Kriterien zum Gefährdungspotential der Fahrbahn:

- Unfallgeschehen,

- Kraftfahrzeugverkehrsstärke und -zusammensetzung sowie

- Kurvigkeit

sowie zu den baulichen Voraussetzungen im Hinblick auf die Benutzungspflicht von Radwegen:

- Flächenverfügbarkeit für Fuß- und Radverkehr,

- Anforderungen an die Verkehrsfläche des Radweges,

- Anforderungen an die Linienführung,

- Anforderungen an Lichtsignalanlagen und Querungseinrichtungen sowie

- Sonderbedingungen für Zweirichtungsradwege

zusammengetragen. Werden die Kriterien an einem vorhandenen Radweg nicht erfüllt, so ist dessen Benutzungspflicht unbegründet. Weiterhin werden Hinweise zur systematischen Überprüfung des Radwegenetzes einer Stadt in Hinblick auf die Benutzungspflicht gegeben, was vorrangig für 
größere Städte mit umfangreicheren Radwegenetzen eine Hilfestellung bietet. Grundlage der Überprüfung der Radwegebenutzungspflicht sind Kenntnisse über oben genannte Attribute der Radverkehrsanlagen, die zum Teil nachträglich erhoben werden müssen. Zudem wird eine räumliche Prioritätenreihung vorgegeben, die in Innenstadtbereichen beginnt, anschließend die Hauptachsen in die Peripherie betrachtet, danach Radwege mit hohem Radverkehrsaufkommen und abschließend alle verbleibenden Radwege berücksichtigt. Knotenpunkte und Querungseinrichtungen haben bei den Prüfungen eine Sonderstellung, da hier die baulichen Mindestanforderungen in jedem Fall einzuhalten sind oder geschaffen werden müssen. Anschließend gilt es aus den ermittelten Radwegparametern die Entscheidung zur Benutzungspflicht zu fällen. Dabei können anhand der definierten Beurteilungskriterien die vier Fälle nach Tabelle 2 auftreten, welche unterschiedliche Bearbeitungsprioritäten bzw. Handlungsbedarfe für geeignete Maßnahmen aufweisen [Klöpfer et al. 2011]. Die entsprechenden Empfehlungen zur Radwegebenutzungspflicht enthält Anhang B.

\begin{tabular}{|c|c|c|c|}
\hline Fall & Gefahrenpotential & $\begin{array}{c}\text { Bauliche } \\
\text { Mindestanforderungen }\end{array}$ & $\begin{array}{c}\text { Notwendigkeit } \\
\text { geeigneter Maßnahmen }\end{array}$ \\
\hline $\mathbf{1}$ & nicht vorhanden & erfüllt & mittel \\
\hline $\mathbf{2}$ & nicht vorhanden & nicht erfüllt & hoch \\
\hline $\mathbf{3}$ & vorhanden & nicht erfüllt & höchste \\
\hline $\mathbf{4}$ & vorhanden & erfüllt & nachrangig \\
\hline
\end{tabular}

Tabelle 2: Mögliche Fälle geprüfter Radverkehrsanlagen und Notwendigkeit geeigneter Maßnahmen nach Mainzer Leitfaden (nach Klöpfer et al. 2011)

Für die Region Hannover wurde ebenfalls ein Bewertungsverfahren anhand einheitlicher Beurteilungskriterien (vorrangig basierend auf den ERA 2010) als Entscheidungsgrundlage für Radverkehrsanlagen entwickelt [SHP 2013]. Das Schema zur Ermittlung einer geeigneten Radverkehrsanlage gemäß diesem Bewertungsverfahren enthält Abbildung 8.

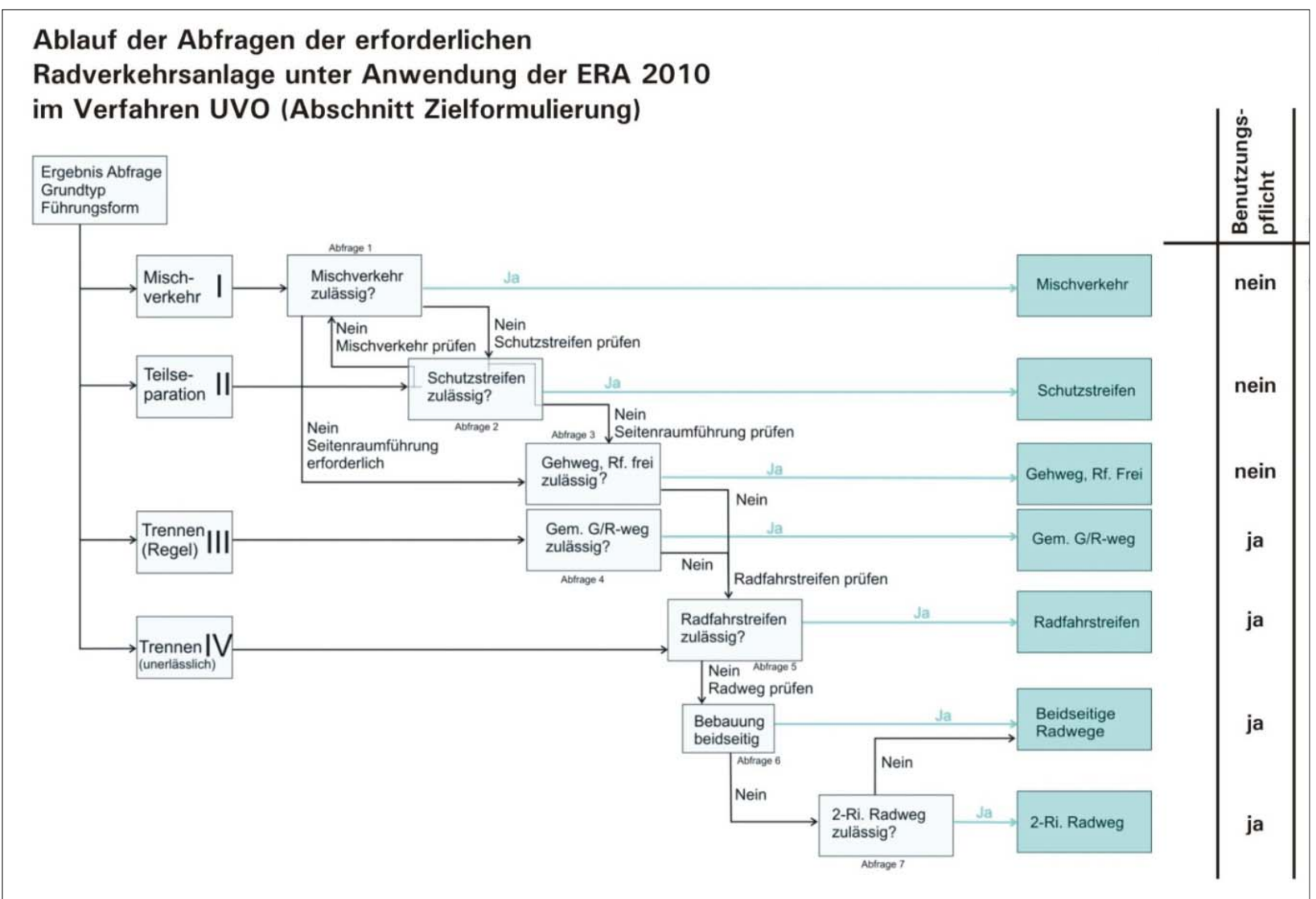

Abbildung 8: Ermittlung erforderlicher Radverkehrsanlagen [SHP 2013] 
Der Ansatz zur Prüfung der Radwegbenutzungspflicht ist dabei, dass zunächst für die zu bewertende Straße theoretisch davon ausgegangen wird, dass keine Radverkehrsanlage vorhanden ist und entsprechend den ERA 2010 eine geeignete Führungsform gefunden werden soll, dies unabhängig davon ob hier derzeit tatsächlich ein Radweg existiert oder nicht. Lautet das Ergebnis der Prüfungen für einen Streckenabschnitt mit vorhandenem benutzungspflichtigem Radweg als Empfehlung „Mischverkehr frei“ oder „Schutzstreifen zulässig“, so sollte die Benutzungspflicht des vorhandenen Radweges aufgehoben werden. Ergibt sich als Empfehlung für eine Straße jedoch „Radweg“, dann gilt hier als mögliche Führungsform das Trennungsprinzip und somit weiterhin die Benutzungspflicht. Die Streckenabschnitte eines Radwegenetzes können mit dem Verfahren einzeln bewertet werden (systematische Prüfung), die Ergebnisse dienen als Empfehlung, jedoch sind auch hier teilweise weitere Einzelfallprüfungen notwendig [SHP 2013].

Neben den beschriebenen Beurteilungskriterien/-verfahren bezüglich der Benutzungspflicht der Radwegeinfrastruktur existieren auch straßenrechtliche Randbedingungen, welche im Zuge der Aufhebung der Radwegbenutzungspflicht zu beachten sind. An Bundes- und Landesstraßen im Zuge von Ortsdurchfahrten kann dabei die Aufhebung der Radwegebenutzungspflicht Auswirkungen auf Planung, Bau, Baulast und Kostentragung einer straßenbegleitenden Radverkehrsanlage haben. Der "Leitfaden Radverkehr" aus Niedersachsen [NLStbV 2013] enthält hierfür weitere Informationen.

Grundlegend gilt bei Aufhebung der Radwegebenutzungspflicht, dass eine verkehrsbehördliche Anordnung nicht unmittelbar eine Änderung der straßenrechtlichen Zuständigkeiten (u.a. Unterhaltung, Verkehrssicherungspflicht) zur Folge hat (vgl. hierzu auch Netter 2014). Ein baulicher Radweg mit Trennung vom Gehweg (z.B. durch Materialwechsel) ist auch nach Aufhebung dessen Benutzungspflicht ein „Vollwertiger" Radweg. Somit obliegt die Baulast weiterhin dem Straßenbaulastträger. Wird ein baulicher Radweg dagegen ganz aufgehoben und beispielsweise als Parkstreifen oder Gehweg umgewidmet, so gilt diese Änderung auch für die straßenrechtliche Baulast. Bei gemeinsamen Geh- und Radwegen ist die Frage nach der entsprechenden Baulast wesentlich differenzierter. Nach der Aufhebung der Benutzungspflicht können sich hier die Zuständigkeiten bei Umwidmung in einen reinen Gehweg oder bei der Anordnung "Gehweg - Radfahrer frei“ unterscheiden [NLStbV 2013].

Der Unterhaltungs- und Erneuerungsbedarf eines nicht benutzungspflichtigen Radweges besteht bei verkehrsplanerischer Notwendigkeit des Radweges und entspricht dem eines benutzungspflichtigen Radweges. Besteht die verkehrsplanerische Notwendigkeit dagegen nicht, so müssen die Anforderungen der betriebssicheren Unterhaltung erfült sein, die Erneuerungen sind dagegen nicht notwendig. Treten am nicht benutzungspflichtigen Radweg Sicherheitsdefizite auf, so sollte den Radfahrern der Eindruck einer Benutzbarkeit des Radweges durch bauliche Änderungen genommen werden [NLStbV 2013].

\subsection{Erfahrungen zu Radverkehrsführungsformen}

Zum Abschluss der Literaturanalyse wird eine Auswahl jüngerer Forschungsarbeiten und Berichte zusammengetragen, welche einen Bezug zur Sicherheit der Führungsformen des Radverkehrs sowie der Aufhebung der Radwegebenutzungspflicht aufweisen. Da konkrete Erkenntnisse zur Sicherheitswirkung der Aufhebung der Radwegebenutzungspflicht bisher selten sind, werden die Schwerpunkte des Unfallgeschehens bei Radverkehrsführungen im Seitenraum und auf der Fahrbahn dargestellt. Dies sind die Führungsformen, deren Sicherheitsaspekte bzw. Besonderheiten bei Aufhebung der Radwegebenutzungspflicht von Bedeutung sind. Abschließend werden zudem vorhandene Erfahrungen zum Nutzungsverhalten von Radfahrern analysiert und aufbereitet. 


\subsubsection{Verkehrssicherheit und Konfliktsituation}

\subsubsection{Erkenntnisse für Streckenabschnitte}

Alrutz et al. [2009] untersuchten aufgrund mehrerer neuer verkehrsrechtlicher Regelungen für den Radverkehr das Sicherheitsniveau und die Nutzung der Führungsformen Radweg mit und ohne Benutzungspflicht, Radfahrstreifen sowie Schutzstreifen (ohne Verkehrsstraßenknotenpunkte). Bei Straßen mit benutzungspflichtigen und nicht benutzungspflichtigen Radwegen liegt in dieser Untersuchung die Unfalldichte doppelt so hoch wie bei markierten Radverkehrsführungen auf der Fahrbahn, zudem streut die Unfalldichte stärker. Die Unfallkostendichte ist dagegen bei Radwegen ohne Benutzungspflicht und Radfahrstreifen nur halb so groß wie bei benutzungspflichtigen Radwegen und liegt bei Schutzstreifen am niedrigsten. Weiterhin werden die Unfalldichten von der Radverkehrsstärke beeinflusst, dagegen nicht von der Kraftfahrzeugverkehrsstärke. Die Tendenzen der Unfallraten entsprechen denen der Unfalldichten, sind aber im Wesentlichen durch die Anteile regelwidrig linksfahrender Radfahrer mitbestimmt. Bei Betrachtung nur rechtsfahrender Radfahrer liegen die Unfallraten bei markierter Fahrbahnführung und nicht benutzungspflichtigen Radwegen auf dem gleichen Niveau. Bei den Unfallkostenraten weisen dagegen benutzungspflichtige Radwege und Radfahrstreifen das größte Risiko auf. Die Unfallkostenraten nicht benutzungspflichtiger Radwege liegen deutlich darunter und erreichen bei Schutzstreifen ein Minimum. Jedoch zeigte sich auch, dass die Ergebnisse zu den einzelnen Führungsformen nicht generalisierbar sind, dagegen eher von baulich-betrieblichen Einzelmerkmalen abhängen [Alrutz et al. 2009].

Bei den Analysen der gegenwärtigen Unfallsituation an 200 Streckenabschnitten (inkl. vorhandener Anschlussknotenpunkte) ergaben aktuellere Regressionsanalysen des GDV [2015] für alle untersuchten Radverkehrsführungen (Fahrbahn, Radweg, Radfahrstreifen, Schutzstreifen) steigende Unfallzahlen mit steigendem Radverkehrsaufkommen. Radwegeführungen besitzen hier das höchste Ausgangsniveau aber den geringsten Anstieg bei steigender Radverkehrsstärke. Mit steigenden Radverkehrsstärken erhöhen sich zudem die Anteile der Unfälle im Längsverkehr, bei Radwegführung betrifft dies zunehmend Unfälle unter Radfahrern selbst. Ferner steigen mit zunehmendem Alter der Radfahrer das Risiko und auch die Schwere von Radunfällen auf Streckenabschnitten. In detaillierteren Analysen zur Führungsform weisen Radwegeführungen leicht höhere Unfallraten auf als die Fahrbahnführung, die nicht benutzungspflichtigen Radwege haben hier die höchste Unfallrate [GDV 2015].

Ohm et al. [2015] betrachteten konkret das Gefährdungspotential der Führung des Radverkehrs im Mischverkehr auf der Fahrbahn differenziert nach dem Vorhandensein von Schutzstreifen. Führungen im Mischverkehr werden aufgrund der Aufhebung der Radwegebenutzungspflicht auch an Hauptverkehrsstraßen künftig zunehmend an Bedeutung gewinnen. Grundlegend weisen hier Personenschadensunfälle im Radverkehr bei Schutzstreifen auf zweistreifigen Querschnitten eine 2,5fach höhere Unfalldichte auf als im reinen Mischverkehr, dabei ist jedoch auch die Anordnung dieser Führungsform in Abhängigkeit der Verkehrsbelastung und einem damit einhergehenden größeren Konfliktpotential zu berücksichtigen. Bei vierstreifigen Straßen besitzt dagegen der reine Mischverkehr eine um Faktor 3 höhere Unfalldichte. Generell ist aber die Unfallschwere bei vorhandenen Schutzstreifen geringer. Weiterhin treten Fahrunfälle und Einbiegen/Kreuzen-Unfälle bei reiner Mischverkehrsführung in den Vordergrund, bei Schutzstreifen dagegen die Abbiegeunfälle. Zudem zeigt sich für beide Führungsformen ein steigendes Unfallgeschehen mit zunehmender Dichte von Einmündungen/Zufahrten, vorhandenen Anlagen des ruhenden Verkehrs und von Straßenbahnen sowie steigender Intensität der Umfeldnutzung [Ohm et al. 2015]. 
Nach Baier et al. [2013] sollte durch die Wahlfreiheit der Flächennutzung der Radfahrer - welche durch die Aufhebung der Radwegebenutzungspflicht mehr und mehr in den Vordergrund rückt auch die Kontinuität einer Radverkehrsanlage bzw. die Häufigkeit der Führungsformwechsel in einem Straßenzug als Sicherheitsindikator berücksichtigt werden.

Alrutz et al. [2009] führten ebenfalls Prüfungen der Unfallentwicklungen mit Aufhebung der Benutzungspflicht von Radwegen ohne weitere bauliche Veränderungen durch. Die Unfalldichten sinken hier nach Aufhebung der Benutzungspflicht stärker als im Vergleich zur Kontrollgruppe. Aufgrund nur geringfügiger Verhaltensanpassungen nach der Aufhebung der Benutzungspflicht (überwiegende Weiternutzung des nicht benutzungspflichtigen Radwegs) waren diese Entwicklungen aber nicht mit der Aufhebung der Benutzungspflicht erklärbar.

\subsubsection{Erkenntnisse Knotenpunkte}

In GDV [2013] wurden Abbiegeunfälle zwischen Kraftfahrzeugen und Radfahrern bei den Führungsformen straßenbegleitender Radweg, Schutz-/Radfahrstreifen und Mischverkehr auf der Fahrbahn analysiert. Das Teilunfallkollektiv der Abbiegeunfälle an Knotenpunkten gehört insgesamt zu den schwersten Radunfällen in Städten (Risiko eines Personenschadens um 10 \% höher als bei allen Radunfällen). 2/3 der Unfälle entfallen hier auf Rechts- und 1/3 auf Linksabbiegevorgänge.

Straßenbegleitende Radwege an Lichtsignalanlagen (LSA) mit mittlerer Furtabsetzung (2 m - $4 \mathrm{~m}$ ) sowie Radwege ohne LSA mit weiter Furtabsetzung ( $>4 \mathrm{~m})$ sind für Rechtsabbiegeunfälle die unfallauffälligsten Führungsformen, oftmals sind hierfür Sichthindernisse im Straßenraum mit ursächlich. Bei Linksabbiegeunfällen sind Radverkehrsführungen im Mischverkehr ohne LSA am risikoreichsten. Unfallursachen bei Abbiegeunfällen sind dabei häufig illegales Linksfahren und regelwidrige Flächen- bzw. Gehwegnutzung (vgl. auch Kapitel 2.5.2) aber auch die Missachtung der Verkehrsregeln an Lichtsignalanlagen [GDV 2013].

Die hohe Unfallgefährdung durch beidseitiges Befahren von Radwegen wird durch die Analysen von Alrutz et al. [2015] zu Zweirichtungsradwegen (vgl. Kapitel 2.1.3) bestätigt. Hier ist das Unfallrisiko bei Fahren in linker Fahrtrichtung doppelt so groß als bei entsprechend rechtsseitiger Fahrweise. Bei regelwidrigem Linksfahren auf Einrichtungsradwegen verdoppelt sich das Unfallrisiko des Fahrens in Gegenrichtung nochmals. Auch trotz möglicher intensiver Sicherungsmaßnahmen muss bei Zweirichtungsradwegen für das Linksfahren von einem erhöhten Grundrisiko gegenüber der rechtsseitigen Fahrweise ausgegangen werden, was entsprechend die Forderungen nach deren Ausnahmestellung weiterhin rechtfertigt [Alrutz et al. 2015].

Bei den Analysen zur Steigerung des Radverkehrsaufkommens konnte in GDV [2015] kein Zusammenhang zwischen höheren Radverkehrsstärken und höherer Unfallbelastung an signalgeregelten Knotenpunkten festgestellt werden. Dagegen steigt das Risiko für Abbiegeunfälle an Radwegen durch zunehmende Radverkehrsgeschwindigkeiten.

\subsubsection{Flächennutzung der Radfahrer}

Bezüglich der Nutzung von Radverkehrsanlagen ergaben sich bei Alrutz et al. [2009] sehr deutliche Ergebnisse. Hier sind im Mittel 90 \% der rechts fahrenden Radfahrer unabhängig von der Art der Radverkehrsanlage (u.a. Radweg mit bzw. ohne Benutzungspflicht) auf der Anlage selbst gefahren. Die Differenzen nach Art der Radverkehrsanlage sind dabei vernachlässigbar gering.

Die Befragungsergebnisse von GDV [2013] zeigen, dass Radwege (unabhängig deren Benutzungspflicht) die präferierte Führungsform im Radverkehr sind (u.a. höherer Fahrkomfort, Sicher- 
heitsempfinden). Schutz-/Radfahrstreifen werden dagegen als schnellste Führungsvariante angesehen. Die Mischverkehrsführung auf der Fahrbahn wird an Knotenpunkten und auch auf der knotenpunktfreien Strecke als unsicherste und unkomfortabelste Lösung empfunden.

Regelwidrige Flächennutzungen rechts fahrender Radfahrer wurden durch Alrutz et al. [2009] bei benutzungspflichtigen Radwegen (im Mittel fuhren hier $2 \%$ auf der Fahrbahn und $6 \%$ auf den Gehwegen) und Radfahrstreifen/Schutzstreifen (7 - $8 \%$ auf Gehwegen, 2 - $3 \%$ auf der Fahrbahn) festgestellt. Ursächlich sind bei markierten Radverkehrsführungen auf der Fahrbahn vorrangig regelwidrige Park-/Liefer- und Ladevorgänge oder Überholvorgänge unter Radfahrern. Auch bei GDV [2013] zeigten sich verschiedene Regelverstöße. Insgesamt fuhren während der Analysen $13 \%$ der Radfahrer nicht auf der vorgesehenen vorhandenen Radverkehrsanlage, sondern auf dem Gehweg rechts daneben, wo sie vom Kraftfahrzeugführer nicht mehr erwartet werden.

Über vergleichbare Regelverstöße berichten auch Ohm et al. [2015]. Vorschriftswidrige Gehwegnutzung bei Mischverkehrsführung wird mit einem Umfang von $2 \%$ bis $17 \%$ angegeben und ist bei vorhandenen Schutzstreifen, breiteren Schutzstreifen und geringen Kraftfahrzeugverkehrsstärken am niedrigsten. Schutzstreifen führen somit zu einer Akzeptanz- und Attraktivitätssteigerung der Fahrbahnnutzung. Eine zulässige Höchstgeschwindigkeit der Kraftfahrzeuge von 30 km/h führt jedoch nicht zwangsläufig zu einer weiteren Verringerung der regelwidrigen Seitenraumnutzung.

Gemäß Alrutz et al. [2009] findet die nach Aufhebung der Radwegebenutzungspflicht erlaubte Fahrbahnnutzung nur zu $4 \%$ statt, eine regelwidrige Gehwegnutzung zu $9 \%$ (meist Überholungen unter Radfahrern bei schmalen Radwegen). Auch wenn die nicht benutzungspflichtigen Radwege nicht die Mindestbreiten aufweisen (teilweise unter $1 \mathrm{~m}$ ), werden sie in großem Umfang genutzt, dies unabhängig von den Verkehrsstärken auf der Fahrbahn. Geringe Tendenzen hin zur Fahrbahnnutzung sind nur bei Hindernissen auf dem Radweg (z.B. Parken) erkennbar. Grundlegend weisen Radwege jedoch die geringste Behinderungsquote auf, die Konfliktrate ist dagegen beeinflusst von anderen Faktoren. Bei nicht benutzungspflichtigen Radwegen zeigt sich hier eine fast 3-fach so hohe Konfliktrate wie bei benutzungspflichtigen Radwegen.

Ursache für die beobachtbare Fahrbahnmeidung der Radfahrer können die Interaktionen zwischen Radfahrern und Kraftfahrzeugführern sein, welche wahrgenommene subjektive Sicherheitsrisiken ergeben können. Hier zeigen Ohm et al. [2015], dass die geforderten seitlichen Sicherheitsabstände von 1,50 m beim Überholen von Radfahrern teilweise bei weitem nicht eingehalten werden. Vor allem bei Gegenverkehr liegen die seitlichen Überholabstände nur bei knapp über 1,30 m. Weiterhin haben Radverkehrsstärken einen direkten Einfluss auf die Häufigkeit von durchgeführten Überholungen und beeinflussen somit die Geschwindigkeit und Verkehrsqualität des Kraftfahrzeugverkehrs. Die geringen Überholabstände häufen sich demnach bei einer Querschnittsbelastung von mehr als $1.000 \mathrm{Kfz} / \mathrm{h}$, über 100 Radfahrer pro Stunde in einer Richtung und weniger als 7,50 $\mathrm{m}$ Fahrbahnbreite.

Als weitere Ursache für die vergleichsweise geringe Nutzung der Fahrbahn bei nicht benutzungspflichtigen Radwegen sehen Alrutz et al. [2009] neben einem höheren subjektiven Sicherheitsgefühl und der Gewohnheit aber auch die Regelunkenntnis an. $70 \%$ der Befragten gehen auch bei nicht benutzungspflichtigen Radwegen von einer Benutzungspflicht aus, da oftmals der Unterschied zwischen beiden Arten von Radwegen nicht bekannt ist. Bezüglich der Radwegebenutzungspflicht stellten GDV [2013] ebenfalls zum Teil erhebliche Wissenslücken fest. Hier gingen $85 \%$ der Befragten davon aus, das eine Radverkehrsanlage bei deren Vorhandensein immer benutzt werden muss, was die Ergebnisse von Alrutz et al. [2009] bestätigt. In den Antworten ergaben sich dabei keine Unterschiede zwischen Kraftfahrzeugführern und Radfahrern. 
Bei Fehlverhalten der Radfahrer existieren gemäß Alrutz et al. [2009] aber auch bewusste Regelverletzungen, dies betrifft vorrangig das Gehwegfahren und das Fahren in Gegenrichtung. Das regelwidrige Linksfahren ist ein generelles Problem von Radwegen. Die Untersuchungen von Alrutz et al. [2015] zeigen, dass auf den meisten beidseitigen Einrichtungsradwegen zwischen 5 und $25 \%$ aller Radfahrer regelwidrig links fährt. Die Anteile regelwidriger Linksfahrer können bei stark einseitig ausgeprägten Zielpunkten für die Radfahrer in Einzelfällen noch größer ausfallen [Alrutz et al. 2015]. In GDV [2013] zeigt sich die Problematik des regelwidrigen Linksfahrens ebenfalls. In der entsprechenden Analyse sind $17 \%$ der Radfahrer in der falschen Richtung gefahren.

Nach Alrutz et al. [2009] zeigt sich regelwidriges Linksfahren zu $20 \%$ der Radverkehrsstärken bei vorhandenen Radwegen (2/3 auf Radweg, 1/3 auf Gehweg). Die verringernden Effekte von regelwidrigem Linksfahren bei Radwegen ohne Benutzungspflicht liegen im Verglich nur bei unter $5 \%$. Bei Radfahrstreifen und Schutzstreifen wird nur zu $10 \%$ in die falsche Richtung gefahren, dies zu über $80 \%$ auf dem Gehweg aber immerhin zu $19 \%$ auf dem Radfahrstreifen oder dem Schutzstreifen (12\%) selbst. Vergleichbare Tendenzen zwischen Radwegen und Fahrbahnführung ergeben sich auch bei GDV [2015], die Anteile regelwidrig linksfahrender Radfahrer sinken aber bei höheren Radverkehrsstärken. Weiterhin fahren vermehrt ältere Radfahrer regelwidrig links.

Neben regelwidrigem Linksfahren gibt es auf Zweirichtungsradwegen auch die Möglichkeit, regelkonform links zu Fahren. Zweirichtungsradwege sollten jedoch innerorts aufgrund der besonderen Unfallgefährdung vermieden werden und stellen eher einen Sonderfall dar (vgl. Kapitel 2.1.3 und 2.5.1). Die Analysen von Alrutz et al. [2015] zeigen, dass bei den untersuchten Zweirichtungsradwegen unabhängig von der Fahrtrichtung und Regelungen zur Benutzungspflicht fast ausschließlich die straßenbegleitenden Radverkehrsanlagen genutzt werden. Dass sich durch die Aufhebung der Radwegebenutzungspflicht hier unterschiedliche Nutzungen ergeben, konnte im Rahmen dieses Projektes aber nicht gesichert festgestellt werden.

\subsubsection{Maßnahmen zur Verbesserung der Radverkehrssicherheit}

Hinsichtlich der betrachteten Anlagentypen ergaben sich bei Alrutz et al. [2009] keine generellen Präferenzen. Weiterhin wird auch die Benutzungspflicht oder deren Verzicht für die Sicherheit von Radwegen nicht als ausschlaggebend angesehen. Stattdessen wird empfohlen, sicherheitsrelevante Anlagenmängel und Entwurfsdefizite zu vermeiden oder zu beseitigen. Aus Sicht der Verkehrssicherheit ist nach Alrutz et al. [2009] die Einhaltung der Sichtfelder an Anschlussknotenpunkten oder stark belasteten Zufahrten sowie die eindeutige Gestaltung von Sicherheitstrennstreifen für eine günstige Sicherheitsbilanz zu gewährleisten.

GDV [2013] empfehlen zur Steigerung der Aufmerksamkeit der Kraftfahrzeugführer an Knotenpunkten eine auffällige und eindeutige Gestaltung von Radwegfurten, wenn mit Radfahrern auf anderen Flächen als der Fahrbahn zu rechnen ist (vorrangig bei nicht benutzungspflichtigen Radwegen). Ähnliches raten GDV [2015] unter Berücksichtigung der demographischen Entwicklung. Einmündungen und stark befahrene Grundstückszufahrten sollten hier mit aufgepflasterten Radwegeüberfahrten ausgebildet werden und über gute Sichtbeziehungen verfügen, da mit älter werdender Bevölkerung ein überproportionaler Anstieg von Einbiegen-/Kreuzen-Unfällen festgestellt wurde [GDV 2015]. Zur Sicherstellung eindeutiger und verständlicher Führungen von Radfahrern sollte weiterhin auf die Anordnung Gehweg Radfahrer frei verzichtet werden. Zudem wird bei hohen Abbiegeverkehrsstärken und Radverkehrsgeschwindigkeiten, komplexen Knotenpunkten und Sichthindernissen eine vollständig signaltechnisch gesicherte Führung empfohlen [GDV 2013].

Jedoch kann an signalgeregelten Knotenpunkten eine potentielle Doppelführung des Radverkehrs bei nicht benutzungspflichtigen Radwegen auch Problemfälle durch die unterschiedliche Signali- 
sierung hervorrufen. Konfliktpotential besteht hier darin, dass die Signale für den Kraftfahrzeugverkehr grundsätzlich länger Grün zeigen. Entsprechend dürfen Radfahrer auf der Fahrbahn noch fahren, während Radfahrer auf dem nicht benutzungspflichtigen Radweg warten müssen. Zudem dürfen rechtsabbiegende Radfahrer auf der Fahrbahn bei Rot nicht fahren, während dies für Radfahrer auf dem Radweg möglich ist [Stadt Münster 2007].

Von GDV [2015] wird darauf hingewiesen, dass eine Höhentrennung zwischen Rad- und Gehweg das regelwidrige Linksfahren von Radfahrern auf Gehwegen reduzieren kann, dies aber nur, wenn ausreichende Radwegbreiten zum Überholen vorhanden sind. Überwachungsmaßnahmen und Ahndung von Fehlverhalten (hier Linksfahren) können sich weiterhin gemäß Alrutz et al. [2009] nur auf Strecken mit hohem Radverkehrsaufkommen konzentrieren und eignen sich nicht als flächendeckende Maßnahmen. Kommunen sollten hier bei der Radverkehrsnetzplanung auf entsprechende Häufungen achten und dort entsprechende Sicherheitsmaßnahmen vorsehen.

Neben infrastrukturellen/betrieblichen Maßnahmen zur Förderung des Fahrbahnfahrens ist auch eine offensive Öffentlichkeitsarbeit zu den geänderten Rahmenbedingungen mit Neuregelung der Radwegebenutzungspflicht erforderlich, da die „Aufhebung der Benutzungspflicht von Radfahrern nicht in jedem Fall als Gewinn, sondern teilweise auch als Einschränkung erlebt wird“" [SHP 2013].

Weiterhin dient die Aufklärungsarbeit zur Förderung der generellen Regelsicherheit, jedoch sind dadurch keine sicherheitsrelevanten Verhaltensänderungen zu erwarten. Zudem sollten die Radfahrer vorrangig auch für radverkehrsanlagenspezifische Gefahrenpunkte sensibilisiert werden [AIrutz et al. 2009]. Dies fordern auch GDV [2015] in Hinblick auf eine alternde Bevölkerung.

\subsubsection{Schlussfolgerungen aus vorhandenen Untersuchungen}

Im Rahmen der vorliegenden Analyse vorhandener Forschungsberichte und Untersuchungen wurden die Sicherheitsbewertungen und Verkehrsverhaltensweisen unterschiedlicher Radverkehrsführungen betrachtet sowie die möglichen Änderungen durch die Aufhebung der Radwegebenutzungspflicht beleuchtet. Bezüglich der analysierten Führungsformen des Radverkehrs gibt es teilweise sehr unterschiedliche Ergebnisse zur Verkehrssicherheit in der vorhandenen Literatur, dabei sind jedoch die Radweglösungen oftmals tendenziell eher auffällig bezüglich des Unfallgeschehens. Die konkreten Veränderungen durch die Aufhebung der Radwegebenutzungspflicht an speziellen Straßen sind hier aber noch nicht in ausreichendem Umfang analysiert worden.

Alrutz et al. [2009] stellten zumindest keine Verschlechterung der Verkehrssicherheit bei Aufhebung der Benutzungspflicht an 10 Radwegen ohne weitere Begleitmaßnahmen fest. Inwieweit die Verkehrssicherheit aber durch Begleitmaßnahmen positiv beeinflussbar ist, konnte hier im VorherNachher-Vergleich nicht beantwortet werden. Weiterhin wurde die Unfallentwicklung unter Berücksichtigung mehrerer verschiedenartiger Begleitmaßnahmen bei Aufhebung der Radwegebenutzungspflicht in keiner der betrachteten Untersuchungen thematisiert. Die Beantwortung dieser Fragestellung ist ein Ziel der vorliegenden Untersuchung.

Für die Entschärfung von Rechtsabbiegekonflikten und -unfällen an Knotenpunkten sowie von weiteren Konflikten an Grundstückszufahrten wäre die durch die Aufhebung der Radwegebenutzungspflicht mögliche Führung von Radfahrern im Mischverkehr eine geeignete Maßnahme. Jedoch sind diese Effekte erheblich von der tatsächlichen Nutzung der Fahrbahn abhängig. Deutliche Verlagerungen von Radfahrern auf die Fahrbahn durch die Aufhebung der Radwegebenutzungspflicht sind aber in den bisherigen Untersuchungen nicht festgestellt worden. Diese geringe Nutzung der Fahrbahn zeigt sich in mehreren Untersuchungen, wie z.B. Alrutz et al. [2009] und GDV [2013]. Weiterhin kann es bei nicht vorhandenen Radwegen auch zu einer regelwidrigen 
Gehwegnutzung kommen (vgl. Ohm et al. [2015]). Die häufige Nutzung des Straßenseitenraumes kann aber bei nicht benutzungspflichtigen oder fehlenden Radwegen aufgrund der potentiellen Doppelführung zur Mischverkehrsführung (unerwartete Radfahrer aus dem Straßenseitenraum) zu Problemen führen. Daher müssen Knotenpunkte im Zuge der Aufhebung der Benutzungspflicht bezüglich der Verkehrssicherheit besonders berücksichtigt werden.

Die häufig festgestellte Seitenraumnutzung lässt grundlegend ein höheres subjektives Sicherheitsempfinden als beim Fahren im Straßenraum vermuten. Hier können u.a. die von Ohm et al. [2015] festgestellten geringen Sicherheitsabstände beim Überholen von Radfahrern auf der Fahrbahn von ca. 1,30 m bei Gegenverkehr - dies unabhängig vom Vorhandensein eines Schutzstreifens - einen entscheidenden Einfluss auf die subjektiven Sicherheitsempfindungen auch bei nicht benutzungspflichtigen Radwegen haben. Welche Begleitmaßnahmen bei der Aufhebung der Radwegebenutzungspflicht die Nutzung der Fahrbahn erhöhen können, stellt ein weiteres Ziel der vorliegenden Untersuchung dar.

Neben den subjektiven Sicherheitsempfindungen besteht aber abschließend auch erheblicher Nachholbedarf bezüglich des Wissens korrekter Verkehrsverhaltensweisen an nicht benutzungspflichtigen Radwegen. Jedoch lässt sich auch nach umfassender Regelvermittlung und der dann vermittelten/bekannten freien Nutzung der nicht benutzungspflichtigen Radwege das Problem der doppelten Flächennutzung nicht beheben. Die geringe Nutzung der Fahrbahn seitens der Radfahrer kann somit weitere Risiken durch unerwartete doppelte Flächennutzungen erzeugen, einem Aspekt, dem ebenfalls im Rahmen der vorliegenden Untersuchung nachgegangen werden soll. 


\section{Befragungen}

Neben der Literaturanalyse wurde zu Projektbeginn eine zweistufige Befragung durchgeführt. In der ersten Phase erfolgte hierfür eine Onlineumfrage in allen deutschen Städten mit mehr als 20.000 Einwohnern, um Erkenntnisse zum Umgang der Kommunen mit den veränderten rechtlichen und planerischen Rahmenbedingungen zur Radwegebenutzungspflicht zusammenzutragen. Die dort erhaltenen Antworten und praxisnahen Erfahrungen stellen eine wesentliche Grundlage für die weiteren Untersuchungen dar.

Zudem wurden - basierend auf den Ergebnissen der ersten Umfragephase - 19 Städte ausgewählt, bei denen weitere detailliertere Informationen zu kommunalen Radverkehrsanlagen im Rahmen einer zweiten telefonischen Umfrage abgefragt wurden. Das Ziel war die Ermittlung konkreter Streckenabschnitte und Radverkehrsanlagen, welche infolge der Neuregelung nicht mehr benutzungspflichtig sind bzw. künftig nicht mehr sein werden. Die hier identifizierten Strecken gingen direkt in die nachfolgende Vorher-Nachher-Analyse des Unfallgeschehens sowie in die Vorher-Nachher-Beobachtung des Verkehrsverhaltens dieses Projektes ein. Die Durchführung und Auswertungen der Onlineumfrage, die Städteauswahl sowie das Vorgehen der weitergehenden telefonischen Befragung sind Bestandteil dieses Kapitels.

\subsection{Erste Umfragephase (Onlineumfrage)}

\subsubsection{Befragungsdurchführung}

Zur Vorbereitung der Onlineumfrage wurden im Gemeindeverzeichnis des Statistischen Bundesamtes zum Gebietsstand 31.03.2015 insgesamt 679 deutsche Städte mit mehr als 20.000 Einwohnern identifiziert [DESTATIS 2015]. Für diese Städte wurde durch eine Onlinerecherche ein Ansprechpartner für die Beantwortung des Fragebogens innerhalb der kommunalen Verwaltung ermittelt. Anschließend wurde ein Onlinefragebogen konzipiert und umgesetzt. Die Umfragedauer umfasste einen 6-wöchigen Zeitraum. Die Ergebnisse der beschriebenen initialen Onlineumfrage werden in nachfolgendem Kapitel 3.1.2 dargestellt.

\subsubsection{Ergebnisse der ersten Umfragephase}

Nach Ende des Befragungszeitraumes konnte auf die Antworten von insgesamt 356 der 679 ursprünglich identifizierten Städte zurückgegriffen werden, dies entspricht einer Rücklaufquote von $52,4 \%$. Die hohe Beteiligung an der Umfrage verdeutlicht die hohe Aktualität und das Interesse der Städte zu Fragen der Radwegebenutzungspflicht.

\subsubsection{Status der kommunalen Radwegebenutzungspflicht}

Frage 1: Halten Sie die Aufhebung der Radwegebenutzungspflicht für sinnvoll?

Beginnend mit der subjektiven Sinnhaftigkeit der Aufhebung der Radwegebenutzungspflicht ergab sich das Ergebnis nach Abbildung 9. 62 \% der Befragten beantworteten diese Frage mit ,ja“, $25 \%$ mit "nein" und die verbleibenden $13 \%$ machten hierzu keine Angaben. Demnach wird die Aufhebung der Radwegebenutzungspflicht in den befragten Städten größtenteils als sinnvolle Maßnahme angesehen. Weiterhin wurde eine Differenzierung der Ergebnisse nach den Bevölkerungszahlen der Städte und somit eine Einteilung in kleine Mittelstädte (20.000 bis 50.000 Einwohner), große Mittelstädte (50.000 bis 100.000 Einwohner) und Großstädte (ab 100.000 Einwohner) gemäß BBSR [2014] vorgenommen. Dabei zeigte sich über die gesamte Auswertung hinweg, dass die Ergebnisse der großen Mittelstädte sehr dicht an den Ergebnissen der Großstädte lagen und der wesentlichste Unterschied hin zu kleinen Mittelstädten bestand. Daher erfolgte die Differenzierung 
nach kleinen Mittelstädten und großen Mittelstädten/Großstädten. Das Ergebnis nach Abbildung 9 zeigt hier einen deutlichen Unterschied. Die Aufhebung der Radwegebenutzungspflicht wird demnach vorrangig in großen Mittelstädten/Großstädten als zweckmäßig erachtet. In kleinen Mittelstädten spricht sich dagegen ein größerer Anteil gegen deren Sinnhaftigkeit aus.
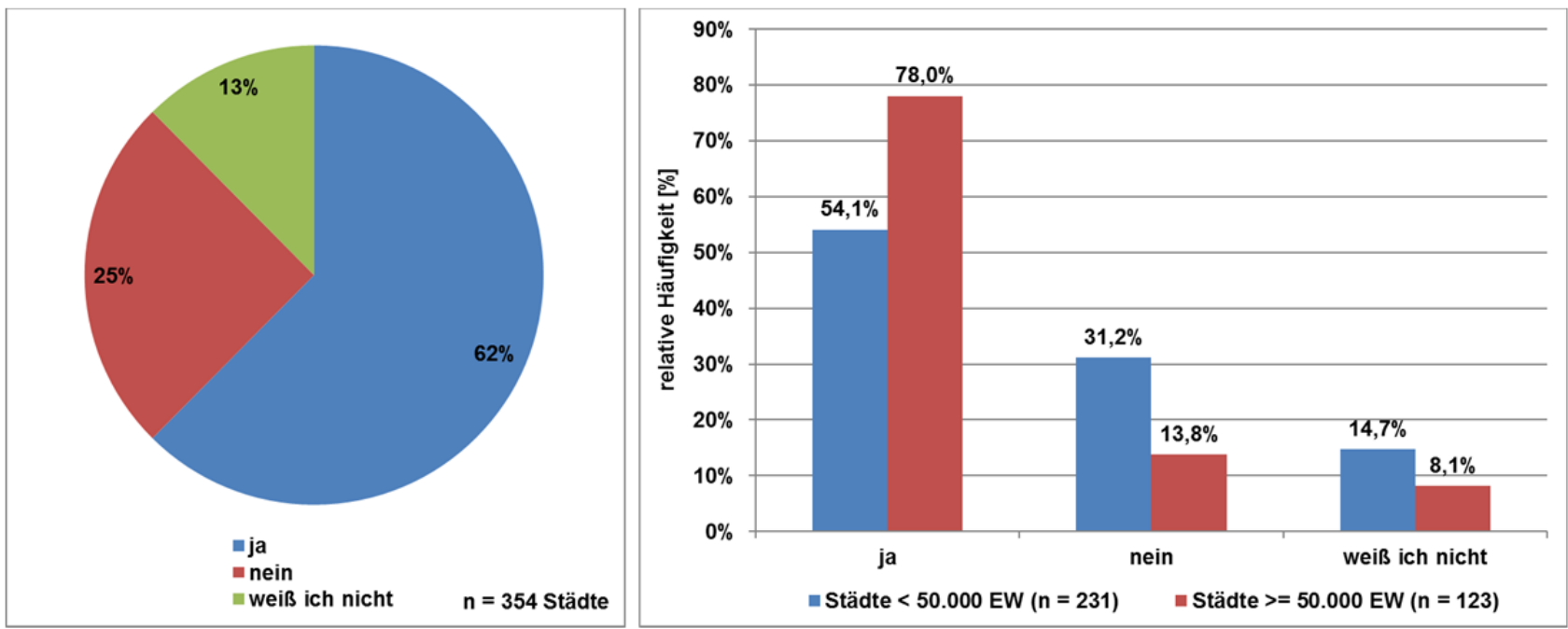

Abbildung 9: Subjektive Sinnhaftigkeit der Aufhebung der Radwegebenutzungspflicht

Frage 2: Gibt es in Ihrer Stadt nicht benutzungspflichtige Radwege? Falls ja, bitte nennen Sie die Länge dieser Radverkehrsanlagen.

Von den insgesamt 329 hier antwortenden Städten gaben $82 \%$ bei dieser Frage „ja“ an, $18 \%$ antworteten dagegen mit "nein“ (vgl. Abbildung 10).
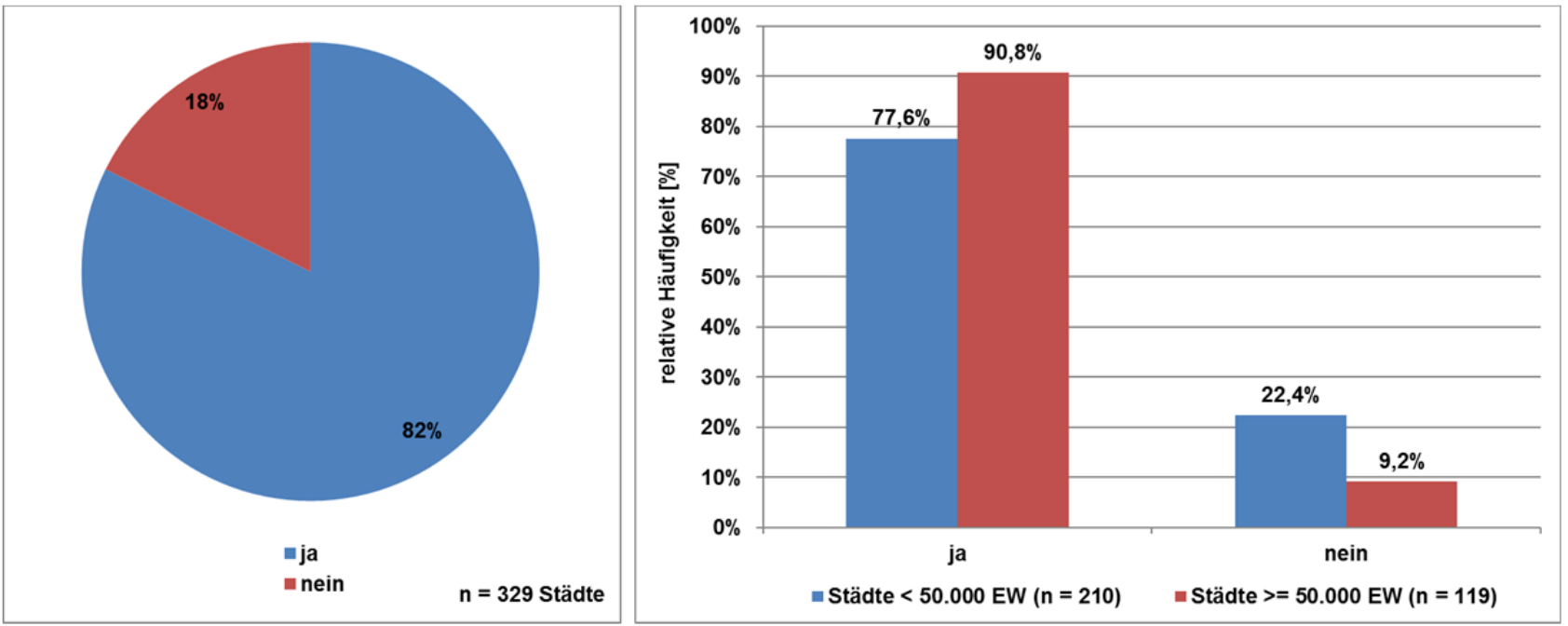

Abbildung 10: Existenz nicht benutzungspflichtiger Radwege in den Städten

Auch bei der Differenzierung nach Einwohnerzahlen ergaben sich die bereits in Abbildung 9 festgestellten Tendenzen. Die Unterschiede sind hier jedoch geringer ausgeprägt. In $22 \%$ der antwortenden kleinen Mittelstädte gibt es keine Radwege ohne Benutzungspflicht, bei größeren Städten sind dies lediglich $9 \%$. Durch die Fragebogengestaltung wurden die Städte, die Frage 2 mit „nein“ beantworteten, direkt zum Ende bzw. zur Dankesseite des Fragebogens weitergeleitet, da diese die weiteren Fragen nicht basierend auf kommunalen Erfahrungen hätten beantworten können. Ergänzende Angaben zur genauen Gesamtlänge der vorhandenen Radwege ohne Benutzungspflicht machten insgesamt nur 33 Städte. Die angegebenen Gesamtlängen schwanken dabei in Abhängigkeit der Stadtgröße von wenigen hundert Metern bis zu mehr als 500 Kilometern. 


\section{Frage 3: Wird bzw. wurde in Ihrer Stadt die Benutzungspflicht der Radwege überprüft?}

Für die o.g. Frage 3 ergaben sich die Ergebnisse nach Abbildung 11. Hier wurde deutlich, dass $7 \%$ der Städte keine Überprüfung der Benutzungspflicht der Radwege durchgeführt haben und dieses zukünftig auch nicht beabsichtigen. Weitere $10 \%$ der antwortenden Städte haben eine derartige Überprüfung geplant aber bisher noch nicht begonnen. Bei $42 \%$ der Städte haben Prüfungen in den vergangenen Jahren begonnen und sind in weiteren $38 \%$ der Fälle bereits abgeschlossen. Demnach sind im Großteil der befragten Städte Überprüfungen der Radwegebenutzungspflicht vorgesehen oder bereits durchgeführt worden.
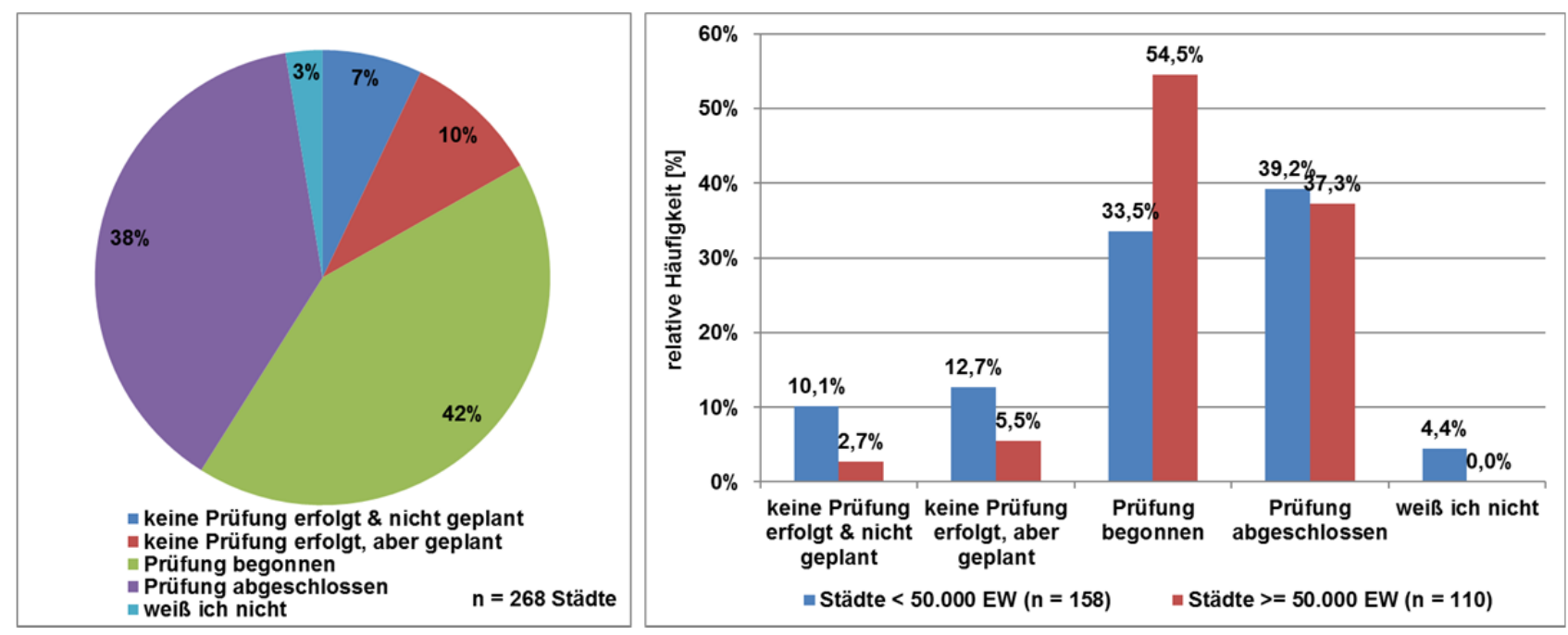

Abbildung 11: Status der Überprüfung der Benutzungspflicht der Radwege in den Städten

Nach Differenzierung der Stadtgröße ergab sich eine Tendenz hin zur Überprüfung der Radwegebenutzungspflicht in Städten ab 50.000 Einwohner. Hier haben in $92 \%$ der Städte Überprüfungen begonnen oder wurden bereits abgeschlossen, in den verbleibenden $8 \%$ der Städte sind die Überprüfungen noch nicht erfolgt. Der Fall, dass Überprüfungen der Radwegebenutzungspflicht bisher nicht erfolgt und künftig auch nicht geplant sind, trat vorrangig in kleinen Mittelstädten auf.

Frage 4: Handelt es sich hierbei um eigen- oder fremdinitiiertes Handlungserfordernis?

Wenn Überprüfungen der Benutzungspflicht von Radwegen geplant sind, aktuell durchgeführt werden oder bereits beendet sind, können diese Handlungen unterschiedlich motiviert sein. Die Antworten auf diese Frage zeigen in Abbildung 12, dass diese Überprüfungen von $70 \%$ der Städte eigeninitiiert (bspw. aufgrund der StVO 2013) durchgeführt werden. Nur in jeder vierten Stadt werden/wurden aufgrund externer Einflüsse fremdinitiierte Überprüfungen der Radwegebenutzungspflicht durchgeführt. Deutliche Unterschiede nach Stadtgrößen waren hier nicht erkennbar.

Handelte es sich um ein fremdinitiiertes Handlungserfordernis, so war dieses entsprechend der folgenden externen Einflüsse motiviert. An erster Stelle stehen hier mit $30 \%$ externe Hinweise oder Anregungen vom Allgemeinen Deutschen Fahrrad-Club (ADFC) sowie vom Verkehrsclub Deutschland (VCD). Darauf folgen mit weiteren $28 \%$ Anweisungen von übergeordneten Verwaltungsbehörden (bspw. Straßenverkehrsbehörden in Kreisverwaltungen). $16 \%$ der Städte mit fremdinitiierten Handlungserfordernis gaben Überprüfungen aufgrund von Bürgeranträgen an, weitere $12 \%$ folgten den Empfehlungen von kommunalen/regionalen Arbeitskreisen. $9 \%$ der Städte führten Überprüfungen aufgrund einer veränderten Gesetzgebung durch. Hier ist jedoch anzunehmen, dass auch ein Großteil der eigeninitiierten kommunalen Handlungen auf derartigen veränderten gesetzlichen Vorgaben (StVO Novellen) beruhen. Die verbleibenden $5 \%$ der Städte nannten Verkehrskonzepte von Ingenieurbüros als Handlungsanreize. Es bleibt festzuhalten, dass 
für die 57 Städte mit ausdrücklich genanntem fremdinitiierten Handlungserfordernis zum Teil sehr unterschiedliche Gründe für die Überprüfung der Radwegebenutzungspflicht existieren.
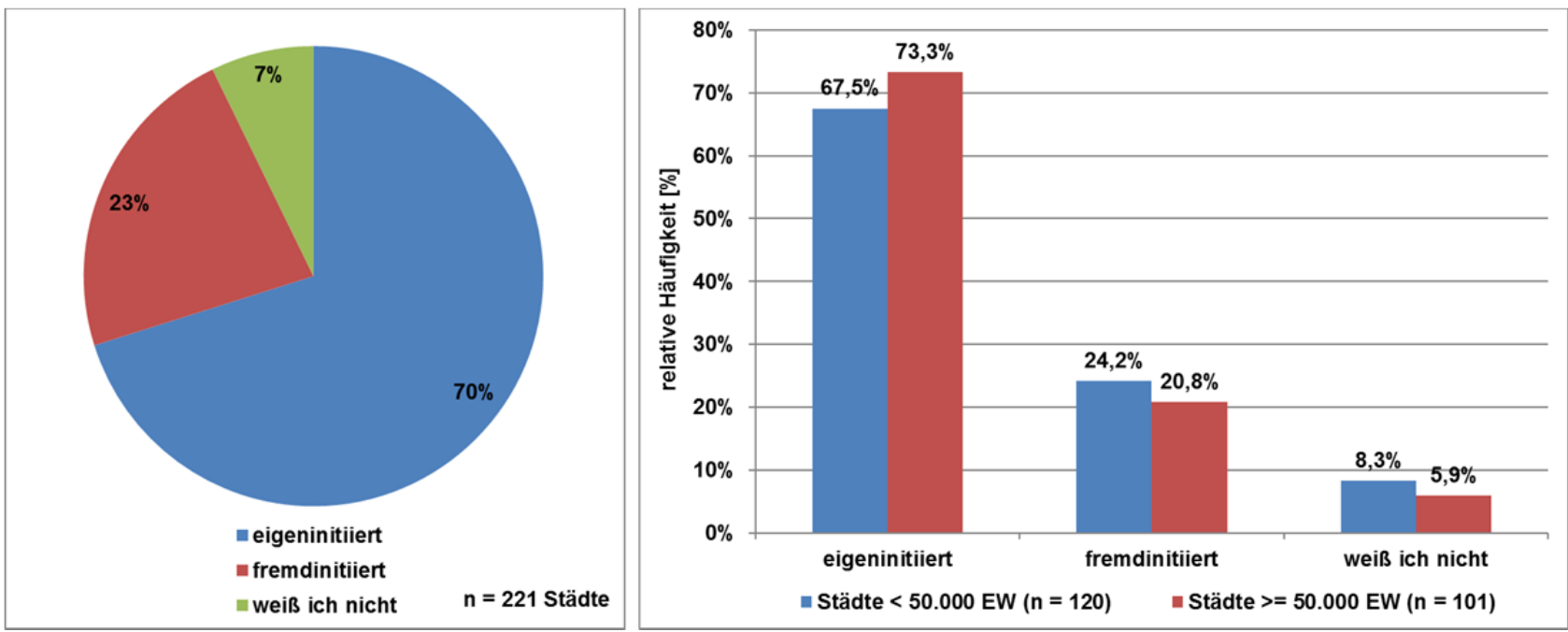

Abbildung 12: Ursachen des Handlungserfordernisses zur Überprüfung der Radwege

Frage 5: Wurde aufgrund der Prüfungen die Radwegebenutzungspflicht in Ihrer Stadt aufgehoben?

In den Antworten auf Frage 5 zeigten sich die Resultate der Überprüfungen der Radwegebenutzungspflicht innerhalb der Städte (vgl. Abbildung 13). Nur $1 \%$ der 216 Städte, die aufgrund der neueren StVO Novellen derartige Überprüfungen durchgeführt haben, sahen basierend auf den entsprechenden Ergebnissen keine Notwendigkeit zur Aufhebung der Radwegebenutzungspflicht. $41 \%$ der Städte heben oder hoben bereits aufgrund der Überprüfungen die Radwegebenutzungspflicht vereinzelt auf. In $42 \%$ der Städte ist die Aufhebung häufig vorgenommen worden. In insgesamt $14 \%$ der Städte wurde die Radwegebenutzungspflicht zudem (fast) vollständig aufgehoben. Bei den Antworten waren aber auch die Stadtgrößen und damit einhergehend die potentielle Anzahl von benutzungspflichtigen Radwegen zu berücksichtigen. Bei dieser Differenzierung zeigte sich jedoch keine eindeutige Tendenz. Lediglich in kleinen Mittelstädten mit potentiell weniger vorhandenen Radwegen wurde die Radwegebenutzungspflicht nach den Prüfungen mit $17 \%$ tendenziell häufiger generell bzw. bis auf wenige Ausnahmen aufgehoben als in Großstädten (8 \%).
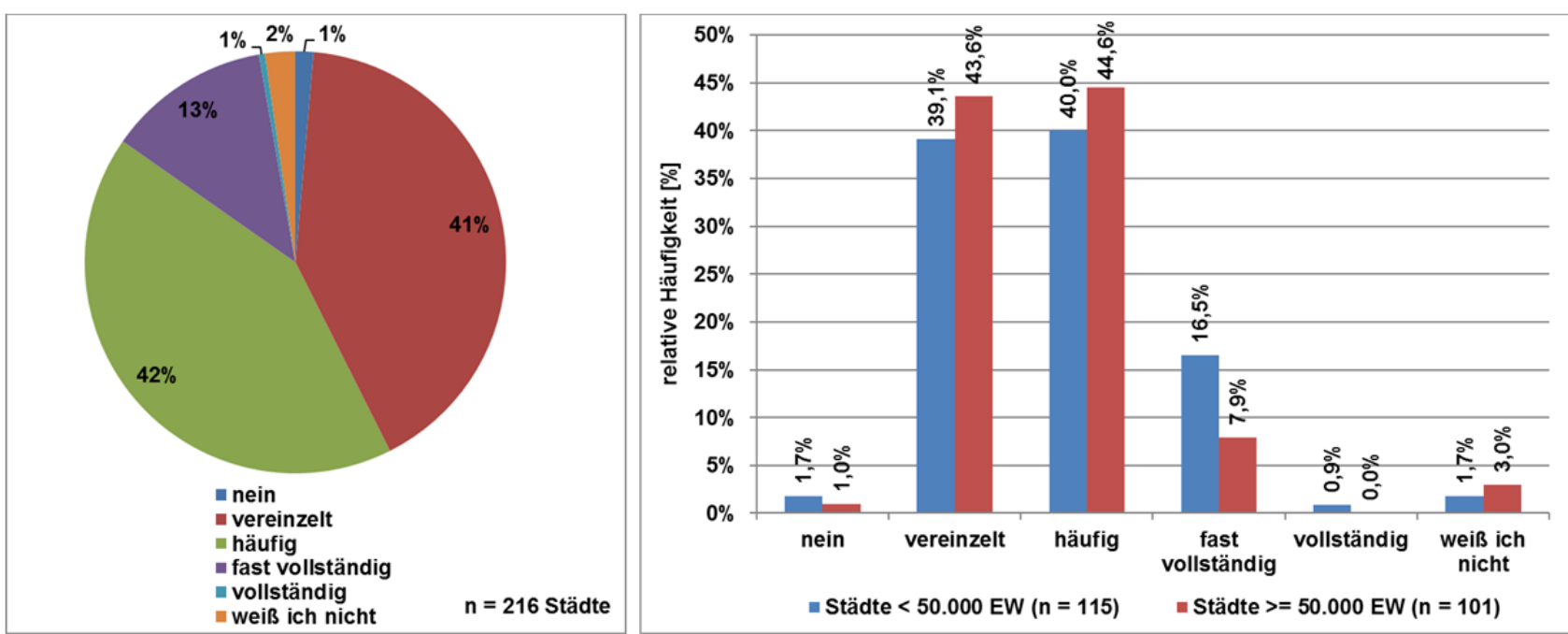

Abbildung 13: Status der Aufhebung der Radwegebenutzungspflicht in den Städten auf Basis der Radwegeüberprüfungen 
Frage 6: Gibt es in Ihrer Stadt ein Programm zur Umsetzung der Aufhebung der Radwegebenutzungspflicht, im Sinne einer systematischen Aufhebung der Benutzungspflicht?

Die o.g. Frage wurde von nur $20 \%$ der Städte mit „ja“ beantwortet, $76 \%$ antworteten mit „nein“ und die verbleibenden $4 \%$ machten zu dieser Frage keine Angaben. Demnach werden die Überprüfungen sowie die Aufhebungen der Radwegebenutzungspflicht überwiegend innerhalb der Kommunen abgearbeitet ohne hierfür im Vorfeld konkretere Konzepte, Herangehensweisen oder Strategien entwickelt zu haben. Jedoch musste auch hier die Stadtgrößen und damit die Anzahl potentiell vorhandener Radverkehrsanlagen beachtet werden. Nach dieser Differenzierung ergab sich ein vergleichsweise deutliches Bild. Wenn Programme zur systematischen Aufhebung der Radwegebenutzungspflicht vorhanden sind, dann handelt es sich hierbei häufiger um Städte mit mehr als 50.000 Einwohnern. Kleine Mittelstädte verfügen dagegen eher selten über derartige Strategien zum Umgang mit den veränderten rechtlichen Rahmenbedingungen in Hinblick auf die Radwegebenutzungspflicht. Strukturierte Programme zur Aufhebung der Radwegebenutzungspflicht sind somit aufgrund der größeren vorhandenen Radwegenetze bzw. -anlagen überwiegend in größeren Städten vorzufinden. In kleinen Mittelstädten mit vorrangig kleinen Radwegenetzen kann die Aufhebung der Radwegebenutzungspflicht dagegen auch eher ohne systematische Herangehensweisen oder entsprechende Konzepte/Programme durchgeführt werden.

Frage 7: Gibt es in Ihrer Stadt zukünftig Planungen für die Aufhebung der Radwegebenutzungspflicht im Hauptstraßennetz?

Bei Frage 7 handelt es sich um ein wesentliches Kriterium für die potentielle Betrachtung einer Stadt innerhalb der im weiteren Projektverlauf vorgesehenen Vorher-Nachher-Analysen zum Verkehrsverhalten. Bei $33 \%$ der 262 hier antwortenden Städte ist in den kommenden Jahren die Aufhebung der Radwegebenutzungspflicht vorgesehen. Bei $55 \%$ der Städte trifft dies nicht zu und $12 \%$ konnten hierzu keine Angaben machen. Nach Differenzierung der Stadtgrößen wurde deutlich, dass künftige Aufhebungen in etwas größerem Umfang in Städten ab 50.000 Einwohnern vorgenommen werden. Somit werden in deutschen Städten in den kommenden Jahren zahlenmäßig weitere nicht benutzungspflichtige Radwege hinzukommen. Für diese können im vorliegenden Projekt die Erfahrungen von bereits bestehenden Anlagen dieser Art zusammengetragen und weitere Handlungsempfehlungen erarbeitet werden.

Frage 8: An wie vielen Radwegen wird die Benutzungspflicht der Radwege in Zukunft aufgehoben?

Aufbauend auf Frage 7 ergab sich die Folgefrage nach der Anzahl der künftig aufzuhebenden Benutzungspflichten von Radwegen. In $14 \%$ der 87 mit ,ja“ auf Frage 7 antwortenden Städte wird die Benutzungspflicht künftig an 1 bis 2 Radwegen aufgehoben. In $18 \%$ der Städte sind 3 bis 5 Radwege und in weiteren $48 \%$ der Städte mehr als 5 Radwege betroffen. Die verbleibenden $20 \%$ konnten hierzu keine Angaben machen. Erwartungsgemäß sind von den künftig umfangreichen Aufhebungen der Radwegebenutzungspflicht (mehr als 5 Radwege) vorrangig größere Städte mit größeren Radwegenetzen betroffen. In kleinen Mittelstädten betrifft dies dagegen überwiegend nur Einzelfälle (weniger als 5 Radwege).

\subsubsection{Begleit- und Unterhaltungsmaßnahmen}

Frage 9: Welche der folgenden Begleitmaßnahmen werden bei der Aufhebung der Benutzungspflicht von Radwegen vorgesehen?

Insgesamt wurde Frage 9 von 257 Städten beantwortet. Davon gaben 54 Städte (21\%) an, dass mit Aufhebung der Radwegebenutzungspflicht grundlegend keine Begleitmaßnahmen umgesetzt 
werden. Demnach wird in diesen Städten nur die Beschilderung, welche im Vorfeld noch die Radwegebenutzungspflicht anordnete, abgebaut. Bei den verbleibenden 203 Städten (79 \%) wurden insgesamt 470 Antworten möglicher Begleitmaßnahmen angegeben. Somit waren pro Stadt mehrere Antworten möglich, sofern hier verschiedene Begleitmaßnahmen mit Aufhebung der Radwegebenutzungspflicht in Frage kommen. Es wird darauf hingewiesen, dass es sich bei den Angaben nur um die reine Nennung möglicher Begleitmaßnahmen in den Städten handelt. Ein Hinweis auf den Anteil von Begleitmaßnahmen in Städten ergibt sich dadurch nicht.

Werden Begleitmaßnahmen umgesetzt, so handelt es sich nach Abbildung 14 häufig um Schutzbzw. Radfahrstreifen auf der Fahrbahn, welche bereits von 73 \% der 203 Städte markiert wurden. Grundlegend muss an dieser Stelle noch auf die Unterschiede beider Maßnahmen hingewiesen werden. Bei der Umsetzung eines Radfahrstreifens handelt es sich erneut um eine benutzungspflichtige Radverkehrsanlage, wodurch die Benutzungspflicht nicht aufgehoben, sondern nur verlagert wird. Bei einem Schutzstreifen handelt es sich dagegen nicht um eine verkehrsbehördliche Anordnung einer Benutzungspflicht.

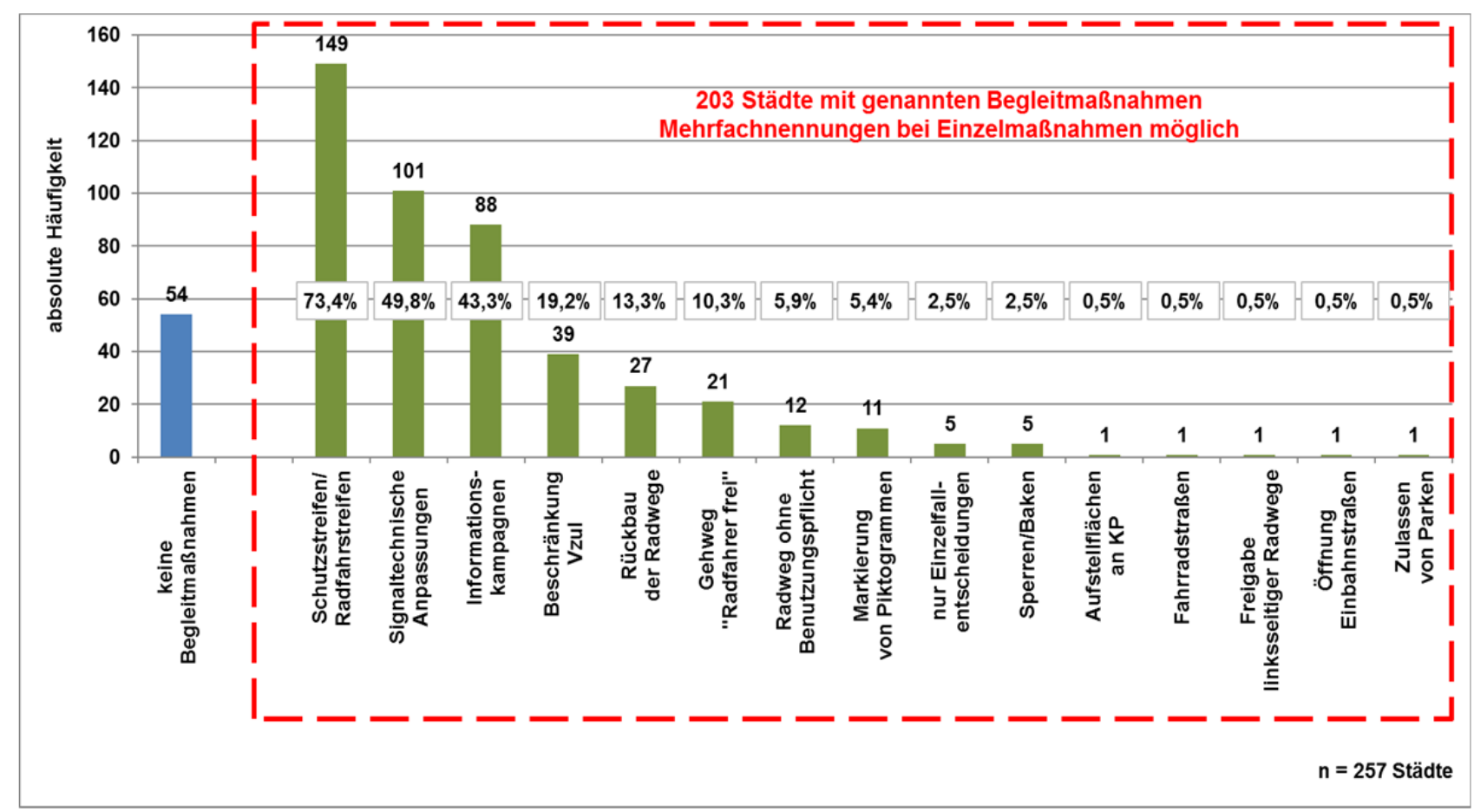

Abbildung 14: Begleitmaßnahmen der Städte mit Aufhebung der Radwegebenutzungspflicht

In $50 \%$ der 203 Städte mit genannten Begleitmaßnahmen wurden signaltechnische Anpassungen an signalisierten Knotenpunkten umgesetzt. Zu deren Notwendigkeit siehe Kapitel 2.3.4. In weiteren $43 \%$ der Städte erfolgen Aufklärungsmaßnahmen der Verkehrsteilnehmer zu den veränderten Rahmenbedingungen. Diese Form der Begleitmaßnahme beinhaltet keine baulichen/betrieblichen Veränderungen, sondern hat nur informativen Charakter, wodurch Hinweise für Radfahrer gegeben und die Akzeptanz der motorisierten Fahrzeugführer erhöht werden soll. Die Öffentlichkeitswirksamkeit der Informationskampagnen ist aber auch von deren Art und Umsetzung abhängig.

Weiterhin wurden in $19 \%$ der Städte vereinzelt Geschwindigkeitsbeschränkungen für den KfzVerkehr angeordnet, wenn Radfahrer die Fahrbahn künftig nutzen dürfen. In weiteren $13 \%$ der Städte wurden bauliche Radwege zurückgebaut. Damit entfällt für Radfahrer sehr restriktiv die Möglichkeit der eigenen Entscheidung zur Nutzung der zur Verfügung stehenden Verkehrsflächen, da ein Benutzungsrecht des ehemaligen benutzungspflichtigen Radweges nicht mehr vorhanden ist. Gleiches gilt für die Anbringung von Sperren oder Baken zur Vermeidung des Radverkehrs auf 
baulich weiterhin vorhandenen Radwegen. Diese Art der Maßnahme wurde aber nur von 5 Städten als mögliche Begleitmaßnahme angegeben und ist eher als Ausnahme zu betrachten.

Weiterhin wurden in $10 \%$ der Städte schon mal anstelle der benutzungspflichtigen Radwege Gehwege mit dem Zusatzzeichen „Radfahrer frei“ ausgewiesen. Somit werden die Verkehrsräume für Fußgänger vergrößert, können aber gleichzeitig durch Radfahrer unter Berücksichtigung der Fußgänger mitbenutzt werden. Ob es sich bei den vorherigen benutzungspflichtigen Radwegen um einen separaten baulichen Radweg oder um einen gemeinsamen Geh- und Radweg handelte, kann mit den hier vorliegenden Befragungsergebnissen nicht ermittelt werden.

$6 \%$ der Städte gaben Radwege ohne Benutzungspflicht als Begleitmaßnahme an. Dabei handelt es sich überwiegend um Radwege mit aufgehobener Benutzungspflicht ohne zusätzliche Begleitmaßnahme. Außerdem wird von einigen wenigen Kommunen durch nichtamtliche Hinweisschilder auf die Radwege ohne Benutzungspflicht hingewiesen und somit verdeutlicht, dass die Fahrbahn durch Radfahrer genutzt werden darf und gleichzeitig auch der Radweg benutzt werden kann.

Zudem wurden in $5 \%$ der Städte schon Piktogramme an nicht benutzungspflichtigen Radwegen als Begleitmaßnahmen markiert. Hier wurden zu gleichen Teilen unterschiedliche Herangehensweisen identifiziert. Teilweise werden Radfahrerpiktogramme am Fahrbahnrand markiert (ohne Schutzstreifen), um Radfahrer und Kraftfahrzeugführer auf das Nutzungsrecht der Fahrbahn durch Radfahrer hinzuweisen. Dagegen werden von einigen Städten auch Markierungen von Radfahrerpiktogrammen auf den nicht mehr benutzungspflichtigen Radwegen vorgenommen, um die Zweckbestimmung des noch vorhandenen Radweges für die Radfahrer hervorzuheben und eventuelles Parken und andere unsachgemäße Nutzungen durch Kraftfahrzeuge zu unterbinden.

In wenigen Fällen wurden Hinweise gegeben, dass es sich bei Begleitmaßnahmen stets um Einzelfallentscheidungen handelt und diese erst nach einem Abwägungsprozess umgesetzt werden. Dieser Aspekt wird jedoch detailliert in der nachfolgenden Frage 10 behandelt. Die verbleibenden angegebenen Begleitmaßnahmen sind ebenfalls lediglich in Einzelfällen aufgetreten. Hierbei handelte es sich um eine Maßnahme, bei der ausdrücklich das Parken auf dem alten nun nicht mehr benutzungspflichtigen Radweg zugelassen wurde. In einem Fall wurde die Öffnung einer Einbahnstraße, in einem weiteren Fall die Freigabe des Radfahrens auf linksseitigen Radwegen als Begleitmaßnahme genannt. In einer Stadt wurden aber auch Aufstellflächen für Radfahrer in Knotenpunktbereichen markiert, um auf die Möglichkeit der Fahrbahnnutzung hinzuweisen. In einem letzten Fall wurde als Begleitmaßnahme für eine aufgehobene Radwegebenutzungspflicht die Straße in eine Fahrradstraße umgewandelt, eine Maßnahme, die besondere Anforderungen benötigt und ebenfalls als Sonderfall zu betrachten ist.

Abschließend bleibt anzumerken, dass neben der Vielzahl der ermittelten verschiedenartigen Begleitmaßnahmen grundsätzlich Begleitmaßnahmen - egal welcher Art - von einigen Städten als zwingend notwendig zu Radwegen mit aufgehobener Benutzungspflicht betrachtet werden.

Frage 10: In welchem Modus werden die von Ihnen ausgewählten Begleitmaßnahmen bei der Aufhebung der Benutzungspflicht von Radwegen durchgeführt?

Aufbauend auf Frage 9 ergibt sich Frage 10 nach dem entsprechenden Modus für eine Auswahl der genannten Begleitmaßnahmen (nur fest vorgegebene Antworten aus Frage 9), demzufolge ob es sich um eine standardmäßige Begleitmaßnahme oder vorrangig eher um Einzelfälle innerhalb der Städte handelt. Es zeigte sich, dass entsprechend der Anmerkungen aus Frage 9 die Vielzahl der Begleitmaßnahmen größtenteils nur einzelfallbezogen angeordnet wird. Standardmäßige Begleitmaßnahmen existieren dagegen nicht. Mit $50 \%$ am häufigsten als Standardfall angegeben wurden Informationskampagnen zur Aufklärung der Verkehrsteilnehmer. Zudem zählen mit $41 \%$ 
signaltechnische Anpassungen zu den Standardverfahrensweisen. Hierbei ist zu beachten, dass Anpassungen nur an Knotenpunkten mit Lichtsignalanlage notwendig werden und diese entsprechend an Radwegen ohne signalgeregelte Knotenpunkte entfallen. Markierungen von Schutz- oder Radfahrstreifen werden in $16 \%$ der Fälle standardmäßig als Begleitmaßnahmen bei Aufhebung der Radwegebenutzungspflicht umgesetzt. Alle weiteren Maßnahmen sind nur als einzelfallbezogene Sonderfälle anzusehen, das Zuzählen zu einem Standardprozedere ist nicht möglich.

\section{Frage 11 und zugehörig Frage 12:}

Welche der folgenden Maßnahmen werden in Ihrer Stadt für Radwege mit und ohne Benutzungspflicht durchgeführt?

Die Fragen 11 und 12 sind einander sehr ähnlich und fragten die verschiedenen Belange von Unterhaltungsmaßnahmen an Radwegen mit und ohne Benutzungspflicht ab. Insgesamt wurde diese Frage von 247 Städten beantwortet. Beim Vergleich der entsprechenden Situation an Radwegen mit und ohne Benutzungspflicht zeigt sich in Abbildung 15 ein klarer Unterschied bei den verschiedenen Unterhaltungsmaßnahmen. Dabei sind ausnahmslos weniger Unterhaltungsmaßnahmen an Radwegen ohne Benutzungspflicht zu erkennen. Grundlegend gelten jedoch für Radwege mit und ohne Benutzungspflicht die gleichen infrastrukturellen und unterhaltungstechnischen Anforderungen. Diese unveränderten Verantwortlichkeiten sind aber mit den seitens der Städte gegebenen Antworten nicht erkennbar. Mögliche Ursachen werden nachfolgend erläutert.

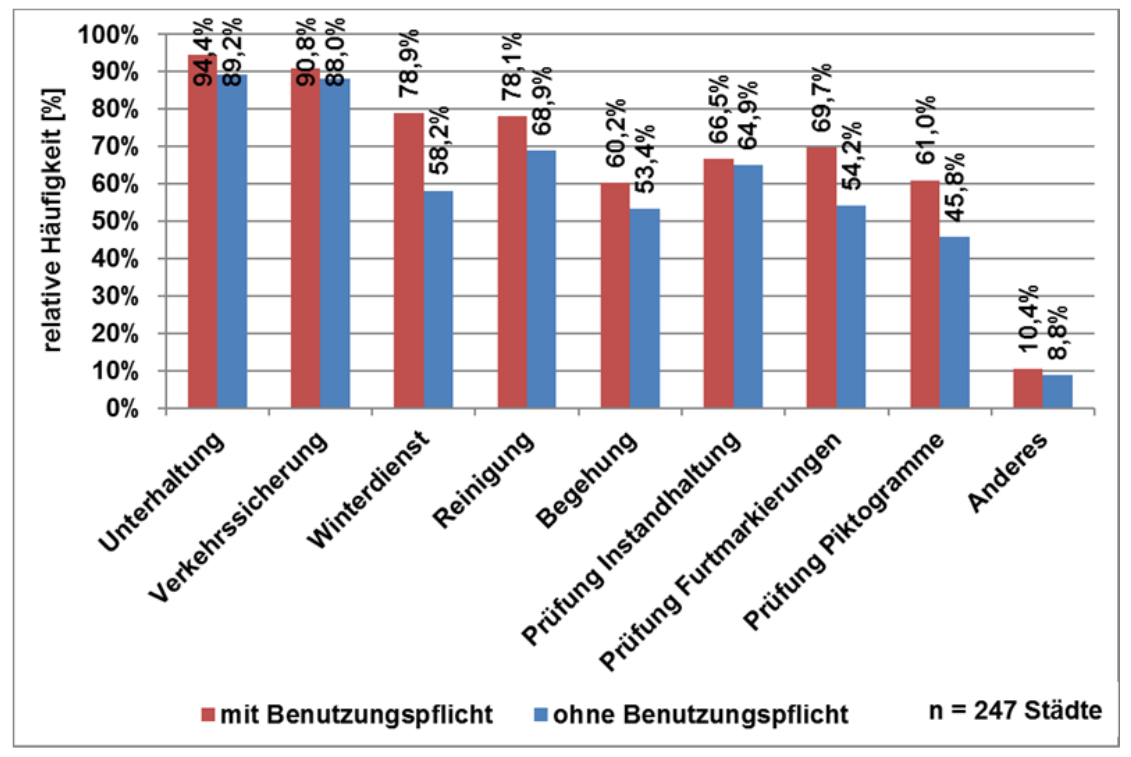

\section{Abbildung 15: Regelmäßig durchgeführte Maßnahmen an Radwegen mit und ohne Benutzungspflicht}

Maßnahmen der Unterhaltung und Verkehrssicherung liegen insgesamt auf dem höchsten Niveau von kontinuierlich durchgeführten Maßnahmen. Zudem sind hier die Unterschiede beim Vergleich von Radwegen mit und ohne Benutzungspflicht am geringsten. Weiterhin führen $79 \%$ der Städte an Radwegen mit Benutzungspflicht regelmäßig Räum- und Streuarbeiten im Winter durch, auf einem ähnlichen Niveau liegen Reinigungsarbeiten. An Radwegen ohne Benutzungspflicht verringert sich dagegen der Anteil der Städte, welche Winter- und Reinigungsdienste durchführen deutlich ${ }^{1}$.

\footnotetext{
${ }^{1}$ Grundlegend obliegen an benutzungspflichtigen und nicht benutzungspflichtigen Radwegen die Winter- und Reinigungsdienste dem Baulastträger. Dennoch können sich z.B. bei Aufhebung der Radwegebenutzungspflicht und der Anordnung Gehweg Radfahrer frei Änderungen ergeben, wenn die Pflichten des Fahrbahnbaulastträgers der Gemeinde [Netter 2014] (bzgl. der Baulast vgl. auch Kapitel 2.4) bzw. die Räumpflichten wiederum den Anliegern (Anliegerräumungspflicht) übertragen werden.
} 
Maßnahmen wie Begehungen, Prüfung der Instandhaltung, der Furtmarkierungen sowie vorhandener Piktogramme werden vergleichsweise von den wenigsten der befragten Städte durchgeführt. Bei der Prüfung von vorhandenen Furtmarkierungen und Piktogrammen ist hier der Unterschied zwischen Radwegen mit und ohne Benutzungspflicht am größten.

Zusammenfassend zeigt sich, dass bei nicht benutzungspflichtigen Radwegen überwiegend nur den Unterhaltungs- und Verkehrssicherungspflichten nachgekommen wird und weitere spezielle Kontrollmaßnahmen seltener durchgeführt werden.

Neben den in Abbildung 15 vorgegebenen Antwortmöglichkeiten wurden von einigen Städten weitere Angaben zur Thematik der Unterhaltung gemacht. Dabei wurde oftmals genannt, dass sich begleitende Radwege in kleineren Kommunen nur an Straßen übergeordneter Funktion befinden und somit die Baulast nicht der Kommune selbst, sondern dem Kreis, Land oder Bund zuzuordnen ist. Dies erklärt, warum nicht alle Städte Unterhaltungs- bzw. Verkehrssicherungspflichten nachkommen. Weiterhin gab es Hinweise, dass Unterhaltung, Sanierung und Instandsetzung nach einer Priorisierung/Rangfolge der Radwege und daraus abgeleitet entsprechend der aktuellen Haushaltslage durchgeführt werden. Zudem wurde auch eine Doppelbelastung der Kommunen an Straßen beispielsweise mit notwendiger Unterhaltung eines Schutzstreifens und des noch vorhandenen nicht benutzungspflichtigen Radweges genannt. Aus Kostengründen findet dann hier keine parallele Unterhaltung beider Radverkehrsanlagen statt, daher erfolgt im Regelfall eine Umnutzung der vorhandenen „anderen Radwege“ um Kosten einzusparen.

\subsubsection{Auswirkungen der Aufhebung der Radwegebenutzungspflicht}

Frage 13: Wie bewerten Sie die Konflikthäufigkeit und Verkehrssicherheit vor und nach der Aufhebung der Radwegebenutzungspflicht?

Mit Frage 13 sollten die Erfahrungen und subjektiven Einschätzungen zu Auswirkungen der Aufhebung der Radwegebenutzungspflicht auf die Konflikthäufigkeit und Verkehrssicherheit ermittelt werden. Insgesamt machten hier 191 Städte entsprechende Angaben. Beide in entgegengesetzte Richtung wirkenden Effekte (steigende Konflikthäufigkeit führt zu geringerer Verkehrssicherheit) wurden auch entsprechend der gegensätzlichen Fragestellungen beantwortet. Die genannten Wirkungen der Aufhebung der Radwegebenutzungspflicht sind identisch und somit konsistent.
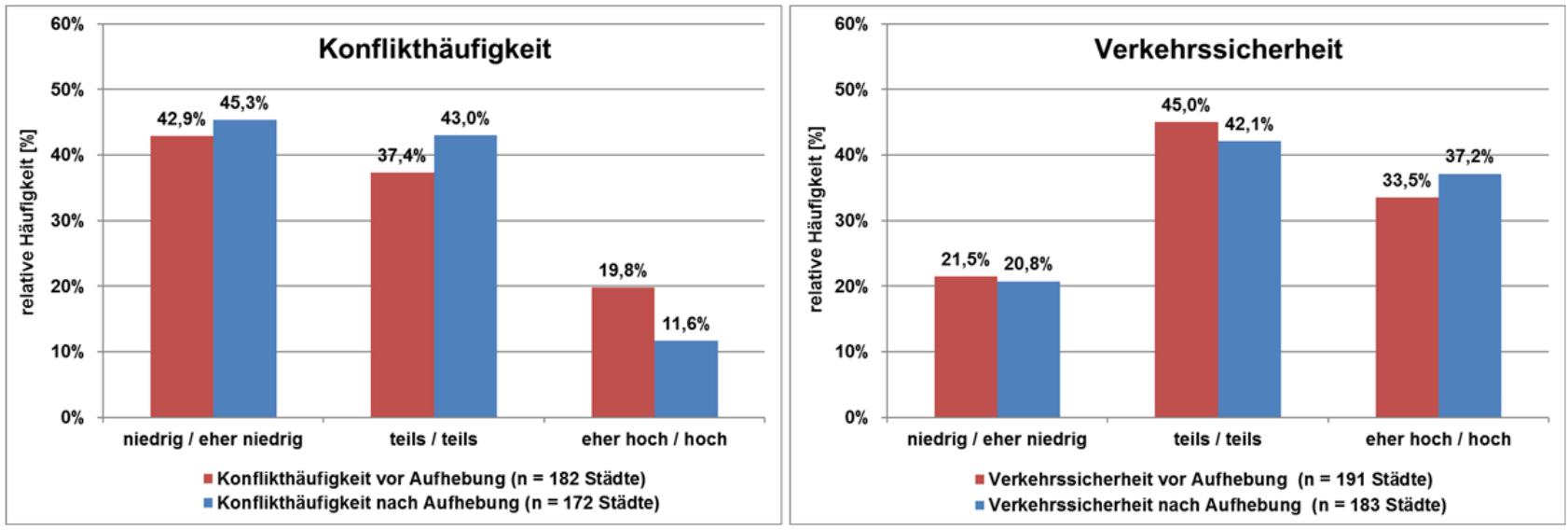

Abbildung 16: Bewertung der Konflikthäufigkeit und der Verkehrssicherheit vor und nach der Aufhebung der Radwegebenutzungspflicht

Beim Vergleich der Bewertungen von Radwegen vor bzw. nach Aufhebung der Benutzungspflicht zeigt sich in Abbildung 16 im Nachher-Fall ein leichter Rückgang der Konflikthäufigkeit. Eine erhöhte Konflikthäufigkeit wird dagegen vor Aufhebung der Radwegebenutzungspflicht gesehen. Bei 
der Verkehrssicherheit sind die Tendenzen entsprechend umgekehrt. Demnach wird von den Befragten innerhalb der Kommunen die Verkehrssicherheit nach Aufhebung der Radwegebenutzungspflicht etwas besser bewertet. Da es sich hierbei jedoch um subjektive Einschätzungen der befragten Personen handelt, sind die aufgezeigten Ergebnisse aber nur als Meinungsbild der entsprechenden Wirkungen anzusehen. Ein deutliches Bild zur Sicherheitslage ergibt sich mit den vorgestellten Ergebnissen eher nicht. Aufgrund der geringen Unterschiede im Vorher-NachherVergleich scheinen außerdem auch sehr gegensätzliche Meinungen zu den Sicherheitswirkungen zu existieren.

Ob die genannten subjektiven Sicherheitsbewertungen dem tatsächlichen Unfallgeschehen und auch den tatsächlichen Konfliktsituationen entsprechen, wird nach den entsprechenden Analysen und Ergebnissen in Kapitel 5 und Kapitel 6 dieses Berichtes beantwortet werden können. Weitere Antworten und Hinweise der Städte zu positiven und negativen Effekten der Aufhebung der Radwegebenutzungspflicht wurden weiterhin mittels der folgenden Fragen 14 und 15 abgefragt.

Frage 14: Welche positiven Effekte (z.B. Reduzierung Abbiegeunfälle) sind Ihrer Meinung nach durch die Aufhebung der Radwegebenutzungspflicht entstanden?

Bei der Abfrage der positiven Effekte durch Aufhebung der Radwegebenutzungspflicht konnte auf die Antworten von 143 Städten mit insgesamt 258 Angaben (Mehrfachantworten möglich) zurückgegriffen werden. Zudem konnten 47 Städte hierzu keine Aussagen treffen und weitere 36 Städte sahen mit der Aufhebung der Radwegebenutzungspflicht keine positiven Effekte, da u.a. Radwege überwiegend weiter genutzt werden. Da bei dieser Frage keine Antwortvorgaben gemacht wurden, mussten die genannten Effekte kategorisiert werden. Bei den Auswertungen der entsprechend im Fragebogen enthaltenen freien Textfelder wurde darauf geachtet, dass die einzelnen hier definierten Kategorien je Stadt nur einmal auftreten. Nachfolgend ist zu beachten, dass die Antworten auf subjektiven Einschätzungen bzw. Vermutungen oder Erfahrungen aus Einzelfällen - jedoch nicht auf konkreten Untersuchungen - beruhen. Die Ergebnisse fasst Abbildung 17 zusammen.

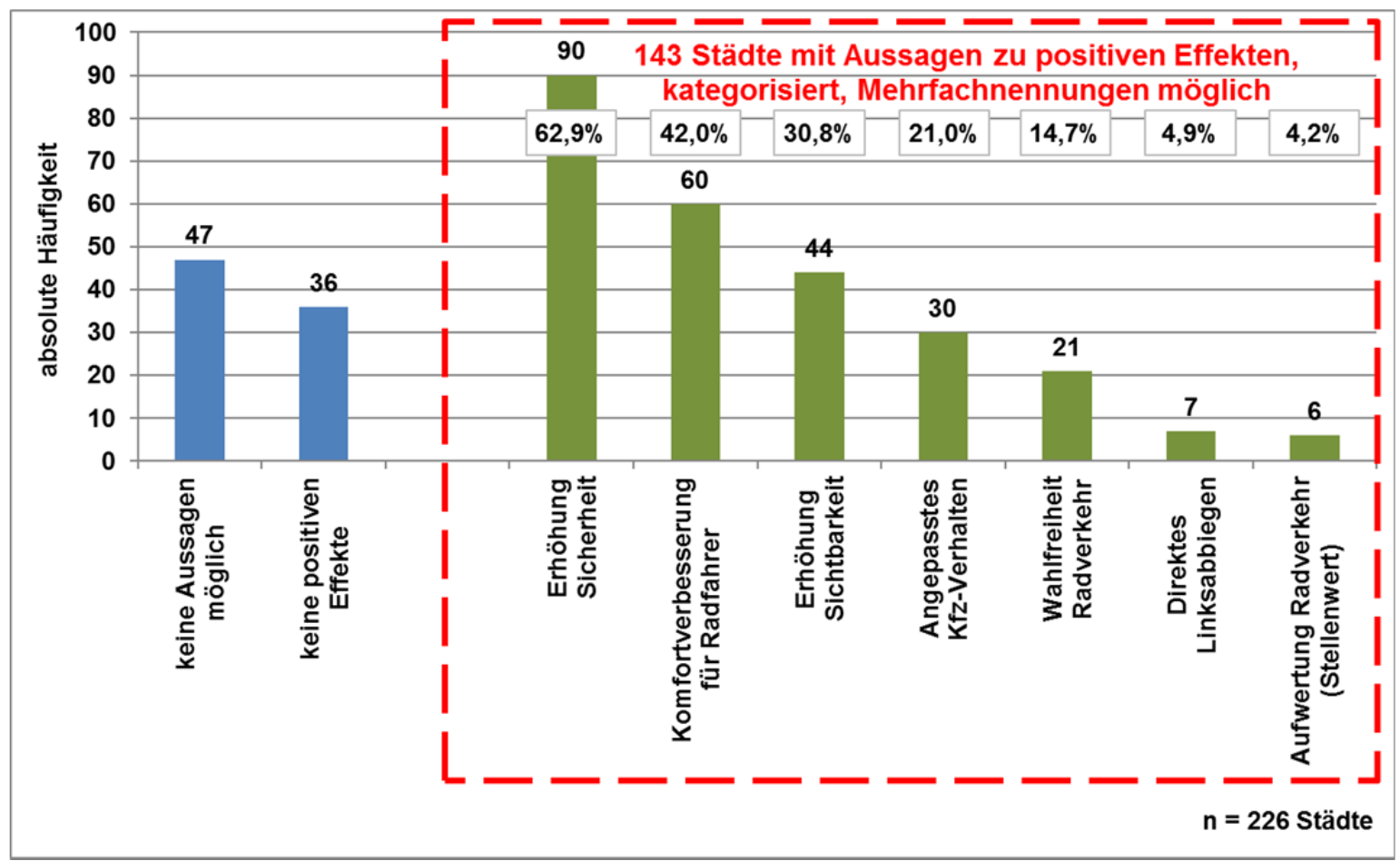

Abbildung 17: Positive Effekte durch die Aufhebung der Radwegebenutzungspflicht 
Als vorrangig positiver Effekt bei Aufhebung der Radwegebenutzungspflicht wurde von $63 \%$ der Städte mit entsprechenden Aussagen die Erhöhung der Verkehrssicherheit genannt. Aus den Freitextantworten wurden dabei die unten zusammengefassten wesentlichen Erläuterungen ermittelt:

- Reduzierung von Abbiege- und Einbiegeunfällen

- Reduzierung der Konflikte an Grundstückszufahrten

- Reduzierung Anteil linksfahrender Radfahrer durch Fahrbahnführung

- Reduzierung von Unfällen mit Fußgängern sowie Fahrgästen an Haltestellen

- Reduzierung von Unfällen zwischen Radfahrern untereinander auf schmalen Radwegen

$42 \%$ der 143 Städte mit genannten positiven Effekten gehen von Verbesserungen des Fahrkomforts für den Radverkehr aus. Als Erläuterungen wurden u.a. angegeben, dass:

- Vorteile für schnelle Radfahrer entstehen,

- langsame und schnelle Radfahrer entmischt und Überholmanöver vermindert werden und

- schmale Radwege oder Radwege in schlechtem Zustand nicht genutzt werden müssen.

31 \% der Städte werteten die Erhöhung der Sichtbarkeit der Radfahrer durch die direkte Fahrbahnführung als positiven Effekt. Als wesentlicher Vorteil wurde hier genannt, dass durch die Führung des Radverkehrs im Sichtfeld der Fahrzeugführer die Sichtbeziehungen verbessert werden, ein Aspekt der vor allem die Verkehrssicherheit beim Abbiegen erhöhen kann. Weitere $21 \%$ der antwortenden Städte mit Erfahrungen zu positiven Effekten nannten Anpassungen im Fahrverhalten der Kraftfahrzeugführer, was durch Reduzierung der Kfz-Geschwindigkeiten, Erhöhung der Aufmerksamkeit sowie vermehrter gegenseitiger Rücksichtnahme erklärt wurde. Abschließend wurde noch die Wahlfreiheit der Radfahrer von $15 \%$, das nun mögliche direkte Linksabbiegen an Knotenpunkten von $5 \%$ und eine Aufwertung des Radverkehrs und somit die Erhöhung dessen Stellenwertes von $4 \%$ der Städte häufiger als positiver Effekt angegeben. Alle eben genannten positiven Effekte können indirekt auch der Verbesserung des Komforts der Radfahrer zugerechnet werden. In Einzelfällen wurde noch die „Entschilderung“ der Städte, geringere Kosten- und Planungsaufwände bei Knotenpunktneubau sowie minimierte Unterhaltungsaufwände für Markierungen und Beschilderungen als positive Effekte genannt. Ein Nachlassen der Instandhaltung von Markierungen auf nicht benutzungspflichtigen Radwegen hatte sich auch schon in Abbildung 15 angedeutet.

Frage 15: Welche negativen Effekte (z.B. neue Konfliktfälle) sind Ihrer Meinung nach durch die Aufhebung der Radwegebenutzungspflicht entstanden?

Bei den negativen Effekten durch Aufhebung der Radwegebenutzungspflicht konnten insgesamt 219 Effekte von 150 Städten mit entsprechenden Erfahrungen ausgewertet werden. Außerdem konnten 37 Städte keine Aussagen zu negativen Effekten machen und weiteren 39 Städten waren derartige Effekte nicht bekannt, was u.a. mit nahezu vollständiger Weiternutzung der nicht benutzungspflichtigen Radwege begründet wurde. Auch hier ist bei der Frageauswertung die Subjektivität der Angaben zu berücksichtigen, dennoch werden wichtige Hinweise auf potentielle negative Einflüsse der Aufhebung der Radwegebenutzungspflicht gegeben.

Für die Hälfte der 150 Städte mit bekannten negativen Effekten (vgl. Abbildung 18) sind Akzeptanzprobleme bei Kraftfahrzeugführern das wesentlichste Problem bei der Aufhebung der Radwegebenutzungspflicht. Den Antworten zufolge ist hierfür die Unkenntnis der rechtlichen Regelungen ursächlich, was bei Kraftfahrzeugführern zu Missverständnissen, Unverständnis, geringer Akzeptanz, Beschwerden und zunehmend aggressivem Fahrverhalten (geringe Sicherheitsabstände, 
Bedrängungen, Nötigungen, Maßregelungen, Hupen, Aggressionsdelikte) führt. Derartige Verhaltensweisen der Fahrzeugführer führen wiederum beim Radverkehr zu einem Unsicherheitsgefühl, was Ursache für die Weiternutzung der nicht benutzungspflichtigen Radwege ist. Von dieser Erfahrung berichteten $47 \%$ der 150 Städte, die u.a. Tempo 50, hohe Kraftfahrzeugverkehrsstärken oder mehrstreifige Stadtstraßen als Ursachen nannten. Neben dem subjektiven Unsicherheitsgefühl wurden zudem für Radfahrer Unsicherheiten über die korrekten Verhaltensweisen genannt, da die Wahlmöglichkeit der Nutzung verschiedener Verkehrsflächen - so bspw. die Mitnutzung der Fahrbahn - häufig unbekannt ist. In Einzelfällen wurde berichtet, dass Radfahrer nach Aufhebung der Radwegebenutzungspflicht sogar von einer Pflicht der Fahrbahnnutzung ausgehen, ein Aspekt der vorrangig an Schulwegen auf Unverständnis in der Bevölkerung stößt. Seitens der Städte wird dabei auch die fehlende Kennzeichnungsmöglichkeit (vgl. Kapitel 2.2.6) für nicht benutzungspflichtige Radwege als Problem angesehen. Zudem wurde angegeben, dass durch die aufgehobene Radwegebenutzungspflicht häufiger auch noch bestehende Radwegebenutzungspflichten missachtet werden. Aber auch trotz durchgeführter Informationskampagnen wurde teilweise von entsprechender Unkenntnis berichtet, da hiermit nicht jeder Verkehrsteilnehmer erreicht werden kann.

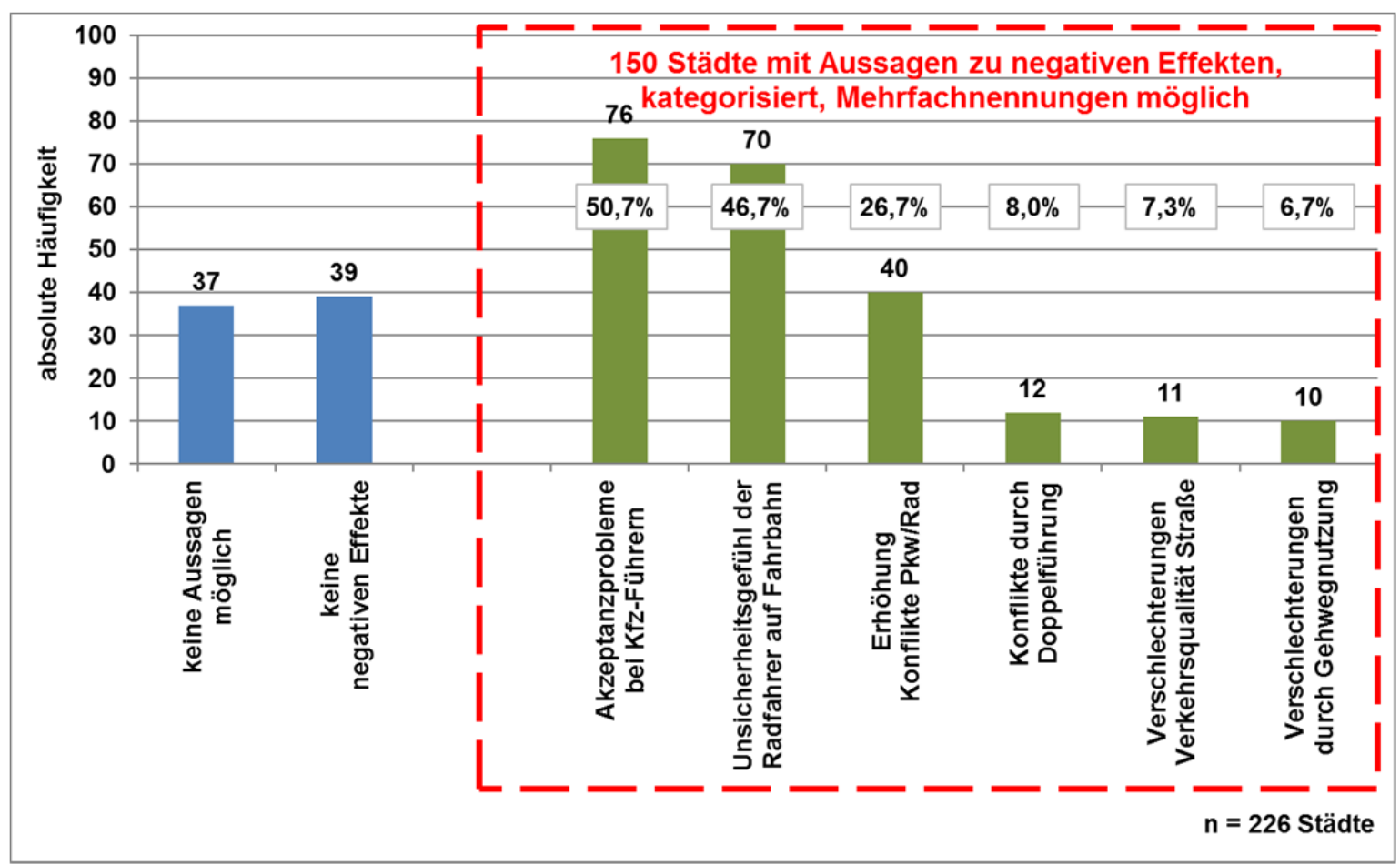

Abbildung 18: Negative Effekte durch die Aufhebung der Radwegebenutzungspflicht

Neben Akzeptanzproblemen und der Unkenntnis von Verkehrsteilnehmern wurde von $27 \%$ der Städte auch die Erhöhung der Konflikte zwischen Kraftfahrzeugen und Radfahrern als negative Auswirkung genannt. Als Problemfälle wurden hier das Überholen, erhöhte Geschwindigkeiten im Kraftfahrzeugstrom sowie Konflikte mit dem ruhenden Verkehr angegeben. Die verbleibenden genannten negativen Effekte sind verglichen mit den bisher beschriebenen Auswirkungen nur vergleichsweise selten genannt worden. Hierzu zählen:

- negative Effekte durch potentielle Doppelführung des Radverkehrs,

- Verschlechterung der Verkehrsqualität von Kraftfahrzeugen und öffentlichem Personennahverkehr auf knotenpunktfreien Abschnitten und Leistungsfähigkeitseinbußen an Knotenpunkten durch erhöhte Zwischenzeiten sowie die

- Nutzung von Gehwegen durch Radfahrer. 
Abschließend wurden vereinzelt die folgenden Kommentare zu negativen Effekten angegeben:

- Unsicherheiten innerhalb der kommunalen Verwaltungen bzgl. Zuständigkeiten

- fehlende klare Handlungsempfehlungen bei Aufhebung der Benutzungspflicht

- Mehrbelastungen der Verwaltungsmitarbeiter

- Mehrkosten durch erforderliche bauliche/betriebliche Anpassungen

- Gefahr des „niedrigeren Unterhaltungsansatzes“ (Vernachlässigung nicht benutzungspflichtiger Radwege)

Die in Frage 14 und 15 vielzähligen positiven und negativen Effekte bestätigen die bereits bei Frage 13 vermuteten sehr unterschiedlichen Sichtweisen zur Aufhebung der Radwegebenutzungspflicht. Auch wenn es sich hierbei nur um subjektive Einschätzungen handelt, konnten verschiedene Grundeinstellungen zur Thematik identifiziert werden. Das vorliegende Projekt soll versuchen, die sicherheitsrelevanten Effekte durch konkrete Untersuchungen zu untermauern.

Frage 16: Wie häufig fahren Ihrer Meinung nach die Radfahrer bei nicht benutzungspflichtigen Radwegen auf der Fahrbahn?

Durch diese Frage erfolgte eine subjektive Einschätzung der Fahrbahnnutzung durch den Radverkehr bei aufgehobener Radwegebenutzungspflicht. Die Antworten der 225 Städte enthält Abbildung 19. Es zeigt sich, dass bei aufgehobener Radwegebenutzungspflicht die Fahrbahn insgesamt zu 47 \% nur „selten“ bzw. „eher selten“ genutzt wird. Zu 40 \% wurde als Einschätzung „teils I teils“ angegeben und lässt hier unterschiedliche Fahrbahnnutzungen in Abhängigkeit weiterer infrastruktureller Randbedingungen vermuten. Zudem wurde das „eher häufige“ bzw. „häufige“ Nutzen der Fahrbahn neben dem nicht benutzungspflichtigen Radweg mit nur $13 \%$ vergleichsweise selten angegeben. Demnach existieren nur wenige Kommunen die tendenziell von einer hohen Fahrbahnnutzung der Radfahrer neben nicht benutzungspflichtigen Radwegen ausgehen. Die bereits bei den negativen Effekten der Aufhebung der Radwegebenutzungspflicht genannten Unsicherheiten bei Nutzung der Verkehrsflächen sowie das erhöhte Unsicherheitsgefühl der Radfahrer im Mischverkehr zeigen sich auch hier. Die Aufhebung der Radwegebenutzungspflicht scheint nach Einschätzung der Befragten offenbar keine deutlichen Verlagerungen des Radverkehrs auf die Fahrbahn zu bewirken.
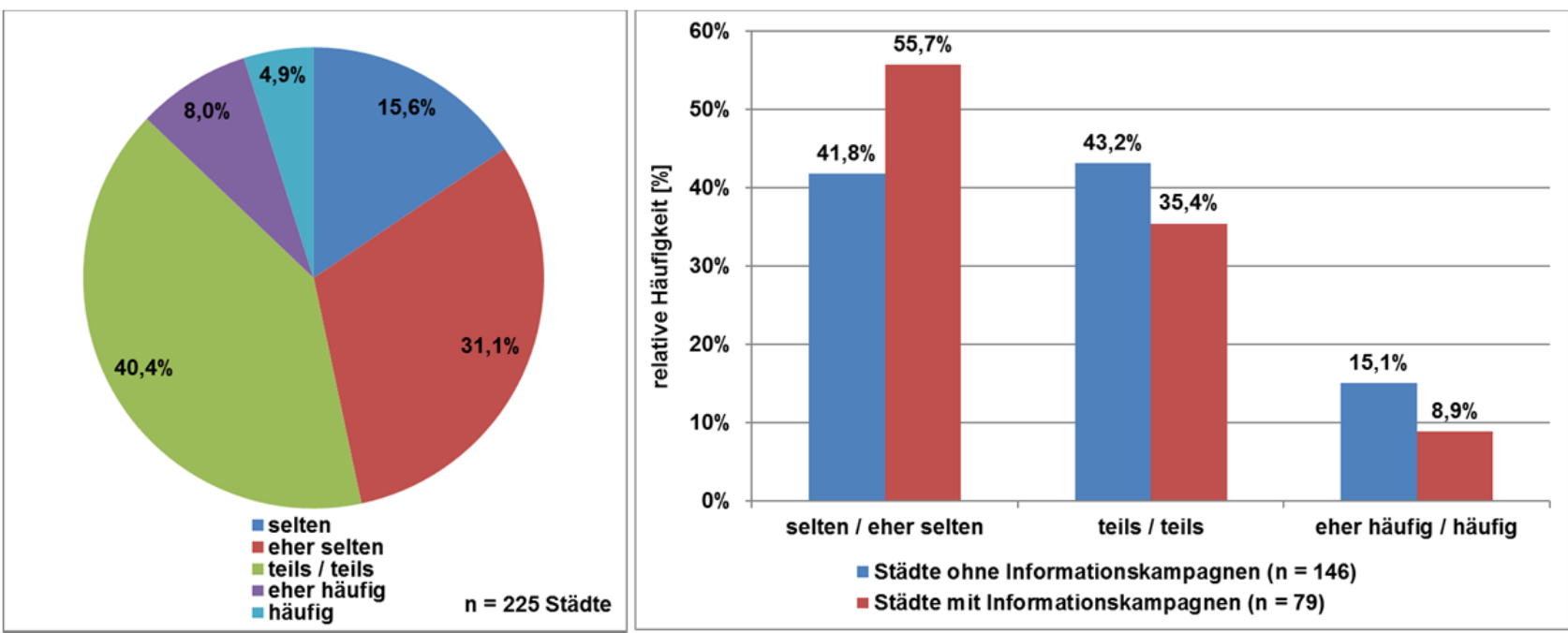

Abbildung 19: Häufigkeit der Fahrbahnnutzung der Radfahrer bei nicht benutzungspflichtigen Radwegen 
Die Differenzierung der Fahrbahnnutzung nach Stadtgrößen zeigte nur schwache Unterschiede, dagegen ergab sich ein interessanter Effekt bei der Differenzierung nach potentiell durchgeführten Informationskampagnen (vgl. Abbildung 19). Hier zeigte sich eine um $14 \%$ seltenere Fahrbahnnutzung durch Radfahrer, wenn in den Städten Informationskampagnen zur Aufhebung der Radwegebenutzungspflicht durchgeführt wurden.

Frage 17: Wie hoch ist Ihrer Meinung nach die Akzeptanz der Kraftfahrzeugführer gegenüber den Radfahrern auf der Fahrbahn bei nicht benutzungspflichtigen Radwegen?

In Anlehnung an Frage 16 ergibt sich eine Folgefrage zur subjektiven Bewertung der Akzeptanz des Radverkehrs auf der Fahrbahn durch Kraftfahrzeugführer. Abbildung 20 zeigt, dass von den 230 hier antwortenden Städten die Akzeptanz durch den Kraftfahrzeugverkehr mit insgesamt $58 \%$ als „niedrig“ bzw. „eher niedrig“ eingeschätzt wurde. Weitere $30 \%$ der Städte konnten dagegen keine eindeutige Bewertung abgeben. Eine „eher hohe“ Akzeptanz der Radfahrer im Mischverkehr durch die Kraftfahrzeugführer wurde von nur $11 \%$ der Städte angegeben, eine hohe Akzeptanz dagegen nie. Somit scheinen, bei der Teilung des Verkehrsraumes der Kraftfahrzeuge mit den Radfahrern, die Kommunen eher von Akzeptanzproblemen auszugehen, was auch die Ergebnisse nach Frage 15 bestätigt. Die Akzeptanzunterschiede zwischen aufgehobener Radwegebenutzungspflicht mit bzw. ohne Informationskampagnen waren vernachlässigbar gering, dagegen war hier die Differenzierung nach der Stadtgröße von höherer Bedeutung. In Städten ab 50.000 Einwohnern wird die Akzeptanz der Radfahrer auf der Fahrbahn geringer bewertet als in kleineren Städten. Demnach sind es überwiegend größere Städte, die mit einer schlechteren Akzeptanz der aufgehobenen Radwegebenutzungspflicht seitens der Kraftfahrzeugführer umgehen müssen.

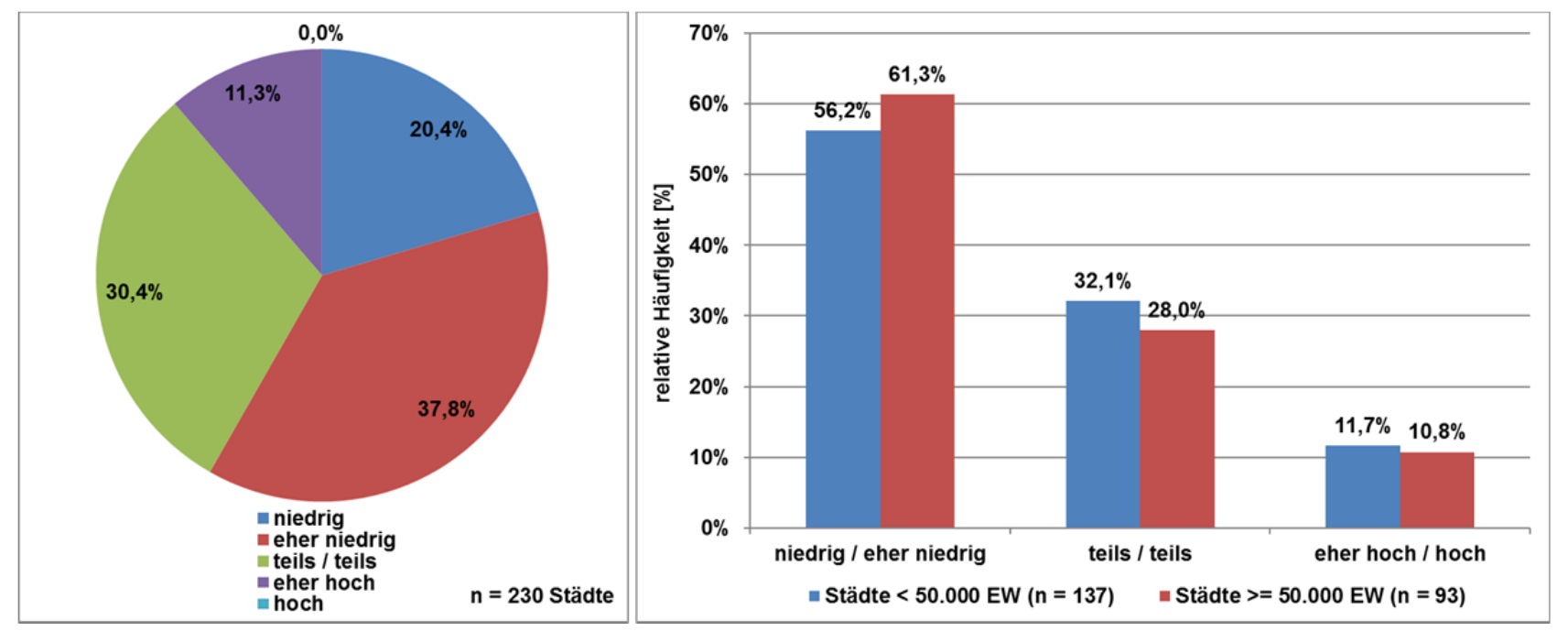

Abbildung 20: Akzeptanz der Kraftfahrzeugführer gegenüber den Radfahrern auf der Fahrbahn bei nicht benutzungspflichtigen Radwegen

Frage 18: Gibt es in Ihrer Stadt nicht benutzungspflichtige Radwege, bei denen auffallend viele Radfahrer auf der Fahrbahn fahren?

Nur $17 \%$ der auf diese Frage antwortenden 237 Städte gaben Radwege mit aufgehobener Benutzungspflicht und erhöhter Fahrbahnnutzung durch den Radverkehr an. In $62 \%$ der Städte sind derartige Radwege nicht vorhanden. Alle weiteren Städte machten hierzu keine Angaben. Grundlegend können durch die genannten Ergebnisse die bereits in Frage 16 festgestellten Tendenzen einer eher geringen Fahrbahnnutzung durch die Radfahrer bestätigt werden. Die Frage spezifiziert jedoch nicht, um wieviel Strecken mit erhöhter Fahrbahnnutzung neben dem nicht benutzungspflichtigen Radweg es sich handelt und unter welchen äußeren Randbedingungen (z.B. Begleit- 
maßnahmen, Verkehrsaufkommen) eine derart vordergründige Straßennutzung festzustellen ist. In den tiefergehenden Befragungen (siehe Kapitel 3.4) gilt es diese Frage nach der erhöhten Fahrbahnnutzung auch für konkrete Untersuchungsbeispiele zu beantworten, um solche Strecken für die weiteren Untersuchungen im Rahmen dieses Projektes zu identifizieren.

Frage 19: Wie hoch ist der Anteil der Radfahrer, welche bei diesen gut akzeptierten nicht benutzungspflichtigen Radwegen auf der Fahrbahn fahren?

Für die 41 mit ,ja“ auf Frage 18 antwortenden Städte wurde eine Folgefrage nach der Einschätzung des Anteils von Radfahrern auf der Fahrbahn gestellt. Hier zeigte sich, dass 21 Städte mit einer Fahrbahnnutzung von weniger als $50 \%$ der Radfahrer antworteten. 19 Städte antworteten mit einer 50 - 99\%igen Nutzung der Fahrbahn durch die Radfahrer. In einer Stadt wurde von einer 100\%igen Fahrbahnnutzung berichtet. Es wird erneut darauf hingewiesen, dass es sich bei den Angaben zu Anteilen der Fahrbahnnutzung nur um einzelne Radwege im entsprechenden Stadtgebiet handeln kann. Diese entsprechenden Nutzungen sind zudem abhängig von den an dem nicht mehr benutzungspflichtigen Radweg vorgenommenen Begleitmaßnahmen. Aufgrund der geringen Stichprobe können die hier erzielten Ergebnisse nicht als maßgebend angesehen werden.

Frage 20 und zugehörig Frage 21:

Gibt es in Ihrer Stadt konkrete Ergebnisse von Untersuchungen zur Aufhebung der

Radwegebenutzungspflicht? In welchem Bereich liegen Untersuchungen vor?

Frage 20 zielte auf bereits von den Kommunen durchgeführte Untersuchungen zur Aufhebung der Radwegebenutzungspflicht ab. Von den 237 antwortenden Städten können lediglich $5 \%$ eigene Untersuchungen zur Thematik vorweisen. Zu $92 \%$ kann auf derartige Untersuchungen nicht zurückgegriffen werden, in den verbleibenden $3 \%$ der Städte ist die Existenz konkreter Untersuchungsergebnisse nicht bekannt. Für die Städte mit verfügbaren Untersuchungen wurden in Frage 21 die Themenbereiche der Untersuchungen abgefragt. Diese betrachteten im Wesentlichen das Konfliktpotential, die Verkehrssicherheit sowie das veränderte Nutzerverhalten bei Aufhebung der Radwegebenutzungspflicht. Die Untersuchungsergebnisse der Städte wurden angefragt und bei entsprechend positiven Rückmeldungen auch in die Literaturanalyse in Kapitel 2 aufgenommen.

\subsubsection{Anordnungskriterien der Radwegebenutzungspflicht}

Frage 22: Laut \& 45 Abs. 9 StVO ist die Radwegebenutzungspflicht anzuordnen, „wenn auf Grund der besonderen örtlichen Verhältnisse eine Gefahrenlage besteht, die das allgemeine Risiko [...] erheblich übersteigt". Bitte nennen und beschreiben Sie die Beurteilungskriterien zur Gefahrenlage in Ihrer Stadt (z.B. Anordnung aufgrund eines bestimmten Unfallgeschehens).

Mit Frage 22 wurden Kriterien sowie deren Beschreibungen/Grenzwerte abgefragt, welche zur Anordnung einer Radwegebenutzungspflicht in den Kommunen aufgrund einer besonderen Gefahrenlage bzw. eines erhöhten Risikos herangezogen werden. Abgesehen von den 17 Städten ohne nähere Angaben wurden die 391 freien Angaben zu Beurteilungskriterien der 140 antwortenden Städte zu Kategorien zusammengefasst. Die Ergebnisse enthält Abbildung 21.

Es zeigt sich, dass $71 \%$ der 140 antwortenden Städte die Verkehrsstärke des Kraftfahrzeugverkehrs sowie $42 \%$ den Schwerverkehrsanteil als Beurteilungskriterien einer Radwegebenutzungspflicht heranziehen. Bei der Beschreibung der Kriterien wurden überwiegend nur allgemeine Erläuterungen - z.B. starkes Verkehrsaufkommen - genannt. Somit scheinen nicht zwangsläufig konkrete Grenzwerte für die Beurteilung der Gefahrenlage verwendet zu werden, was auch in wenigen Fällen direkt angegeben wurde. Zudem wurden auch folgende konkretere Kriterien für die Anord- 
nung einer Radwegebenutzungspflicht angegeben. Überwiegend handelte es sich dabei um den Grenzwert von $10.000 \mathrm{Kfz} / 24 \mathrm{~h}$, jedoch wurden auch $5.000 \mathrm{Kfz} / 24 \mathrm{~h}$ und $15.000 \mathrm{Kfz} / 24 \mathrm{~h}$ sowie Spitzenstundenbelastungen größer $1.000 \mathrm{Kfz}$ bzw. $1.800 \mathrm{Kfz}$ als Grenzwerte genannt. Für den Schwerverkehr wurde ein Anteil größer $10 \%$ sowie mehr als 300 SV/Tag bzW. 1.000 SV/Tag angegeben. Mehrfach wurde auch auf die Empfehlungen der RASt/ERA (vgl. Kapitel 2.3) verwiesen.

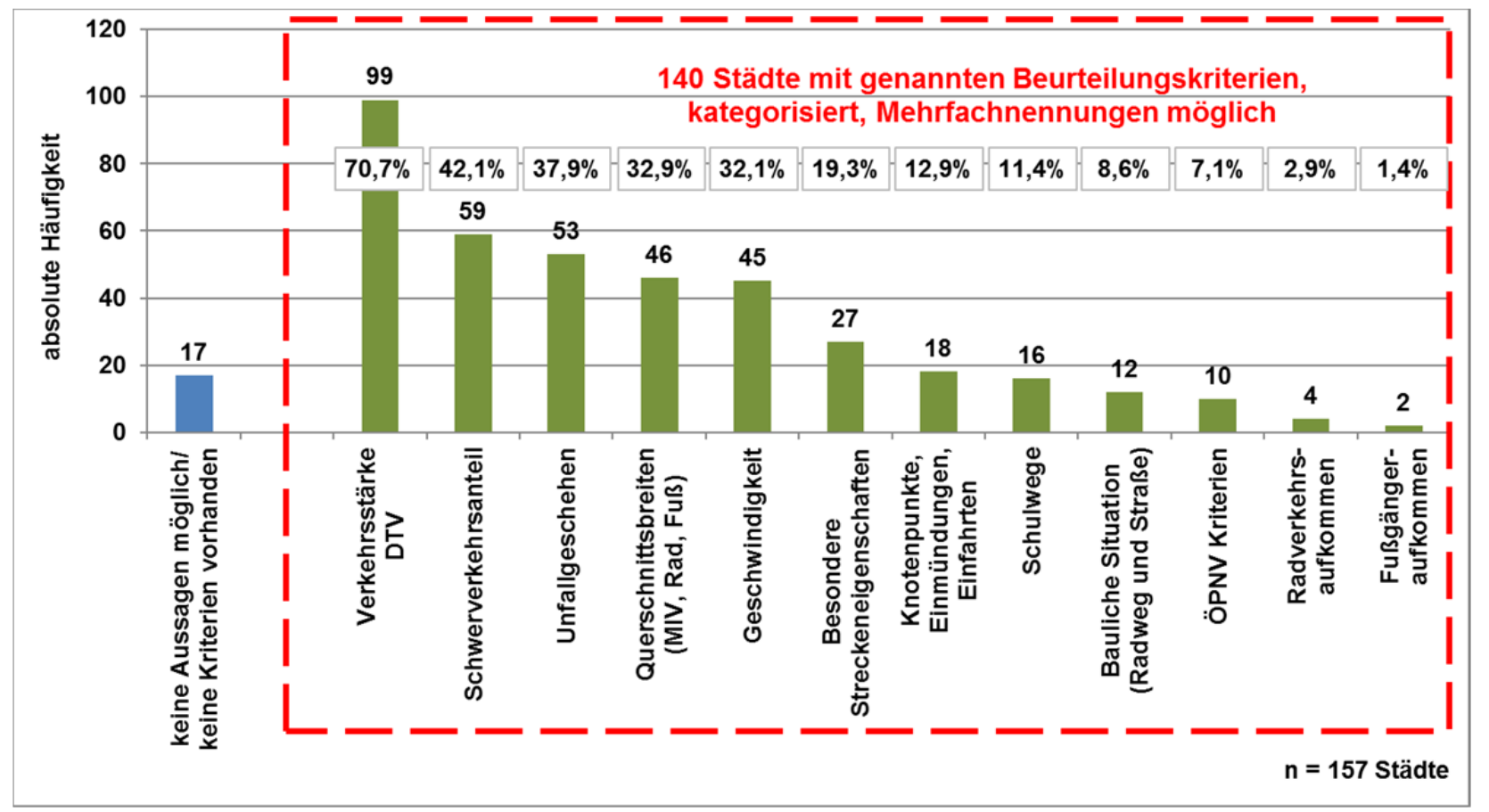

\section{Abbildung 21: Kriterien zur Beurteilung der Gefahrenlage bei Entscheidung über die Benutzungspflicht der Radwege}

Als weiteres Beurteilungskriterium der Gefahrenlage wurde das Unfallgeschehen von $38 \%$ der Städte sowie das Vorhandensein von Unfallhäufungsstellen mit Radfahrerbeteiligung angegeben. Dies ist jedoch nur eine reaktive Maßnahme. Über die Verkehrsstärke kann im Gegensatz dazu auch präventiv zur Unfallvermeidung durch Anordnung einer Radwegebenutzungspflicht beigetragen werden. Weiterhin verwenden $33 \%$ der Städte die verfügbaren Querschnittsbreiten des Straßenraumes (Fahrstreifenbreite und -anzahl, Rad- und Gehwegbreite) sowie die entsprechend vorgegebene Mindestbreiten als Beurteilungskriterium für die Radwegebenutzungspflicht. Zudem wird von $32 \%$ der Städte die zulässige Höchstgeschwindigkeit (aber auch teilweise die tatsächlich gefahrene Geschwindigkeit) auf der Fahrbahn herangezogen. Bezüglich der Grenzwerte wurde hierbei vielfach auf die definierten Belastungsbereiche der ERA [2010] (vgl. Abbildung 6) verwiesen.

Die nachfolgenden Beurteilungskriterien der Radwegbenutzungspflicht aufgrund einer besonderen Gefährdungssituation wurden nur von höchstens 20 \% der Städte genannt (vgl. Abbildung 21):

- besondere Streckeneigenschaften (Engstellen, Steigungs- und Gefällestrecken, kurvige Straßen, schlechte Sichtverhältnisse)

- Komplexität und Anzahl von Knotenpunkten, Einmündungen, Grundstücksfahrten

- Schulwege zur Förderung gesicherter Führungen von Schülern

- bauliche Situation des Radweges und der Fahrbahn

- Öffentlicher Personennahverkehr (Straßenbahngleise, Takt- und Haltestellendichte)

- Radverkehrs- und Fußgängeraufkommen 
Ergänzend zu obenstehenden Kriterien wurden zur Anordnung der Radwegebenutzungspflicht in Einzelfällen noch vorhandene „Windschneisen“, die Vielzahl von erreichbaren Zielen des Radverkehrs im Straßenseitenraum oder Häufungen von zu tiefliegenden Straßenabläufen genannt.

Frage 23: Weiterhin ist die Anordnung einer Radwegebenutzungspflicht vorzunehmen, wenn es der Verkehrsablauf erfordert (VwV-StVO zu \& 2 Absatz 4 Satz 2). Nennen und beschreiben Sie die Beurteilungskriterien des Verkehrsablaufs in Ihrer Stadt (z.B. Anordnung aufgrund einer bestimmten Verkehrsstärke)?

Neben der besonderen Gefahrenlage gelten nach VwV-StVO [2015] auch der Verkehrsablauf bzw. die Verkehrsqualität als Anordnungskriterium für die Radwegebenutzungspflicht. Die entsprechenden 214 genannten Kriterien der 115 antwortenden Städte sind kategorisiert in Abbildung 22 dargestellt. Darüber hinaus wurden von weiteren 15 Städten keine Aussagen zu entsprechenden Beurteilungskriterien getätigt.

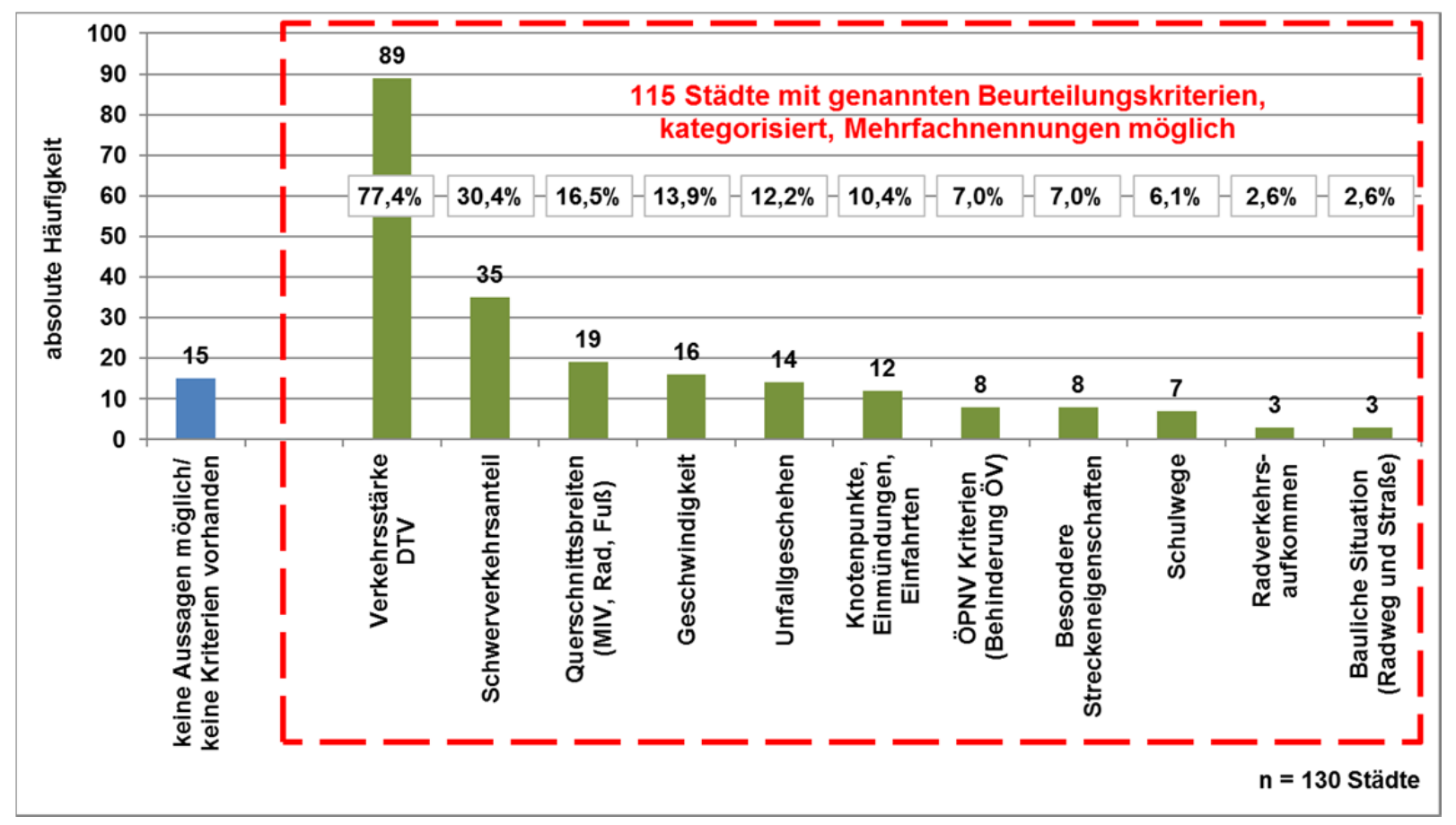

Abbildung 22: Kriterien zur Beurteilung der Benutzungspflicht von Radwege aufgrund der Verkehrsqualität

Ähnlich der besonderen Gefahrenlage werden als wesentliche Kriterien für den Verkehrsablauf die Verkehrsstärke von $77 \%$ der Städte und der Schwerverkehrsanteil von $30 \%$ der Städte zur Beurteilung herangezogen. Dabei sind auch grundlegend die in Frage 22 genannten Grenzwerte ähnlich. Demnach wird aus Gründen des Verkehrsablaufs die Radwegebenutzungspflicht angeordnet, wenn bestimmte Verkehrsstärken (größer 5.000/8.000/10.000/15.000 Kfz/24h bzw. mehr als 1.000/1.200 Kfz in der Spitzenstunde) oder Schwerverkehrsanteile (größer $10 \%$ ) überschritten werden. Auch hier werden die Empfehlungen geltender Regelwerke (RASt, ERA) oftmals als Orientierung herangezogen. Im Vergleich zur Verkehrsstärke und -zusammensetzung sind die weiteren Beurteilungskriterien in Hinblick auf den Verkehrsablauf eher untergeordnet. Diese sind:

- Querschnittsbreiten der Fahrbahn (in $17 \%$ der Städte)

- Geschwindigkeiten des Kraftfahrzeugverkehrs (in $14 \%$ der Städte)

- vorhandenes Unfallgeschehen (in $12 \%$ der Städte)

- Leistungsfähigkeit großer, hochbelasteter Knotenpunkte (in 10 \% der Städte) 
- Öffentlicher Personennahverkehr (in $7 \%$ der Städte)

- besondere Streckeneigenschaften (in $6 \%$ der Städte)

- Schulwege (in $6 \%$ der Städte)

- Radverkehrsaufkommen sowie baulicher Radwegzustand (jeweils unter 3 \% der Städte)

Es bleibt festzuhalten, dass sich die genannten Anordnungskriterien für benutzungspflichtige Radwege aus Gründen der Verkehrssicherheit und Verkehrsqualität sehr ähneln. Als maßgebend für die Anordnung der Radwegebenutzungspflicht wird jedoch grundlegend die Verkehrssicherheit angesehen, da hier nahezu doppelt so viele Kriterien genannt wurden wie für die Verkehrsqualität und weiterhin auch zwischen den Kriterien der Gefahrenlage und Verkehrsqualität gewisse Parallelen bestehen. Dies käme auch der Forderung der VwV-StVO, dass die Verkehrssicherheit aller Verkehrsteilnehmer der Flüssigkeit des Verkehrs vorzuziehen ist, gleich (vgl. Kapitel 2.1.3).

Frage 24: Wurde in Ihrer Stadt bereits gegen bestehende Benutzungspflichten von Radwegen geklagt?

Abschließend war mit dieser Frage zu klären, ob gegen die Anordnung der Radwegbenutzungspflicht in den Städten in Einzelfällen bereits gerichtlich vorgegangen wurde. Die abgegebenen Antworten aus insgesamt 234 Städten zeigen, dass im Großteil der Städte mit $82 \%$ bisher keine Klagen gegen bestehende Radwegebenutzungspflichten vorliegen, $5 \%$ der Städte konnten hierzu keine Angaben machen. In den anderen $13 \%$ (entspricht insgesamt 31 Städten) wurde dagegen bereits gegen vorhandene Radwegebenutzungspflichten geklagt und entsprechend rechtliche Schritte eingeleitet.

\subsubsection{Bereitschaft zur zweiten Umfragephase}

Frage 26: Im Rahmen der Projektbearbeitung ist eine zweite Befragung in Kommunen zu konkreten Umsetzungsbeispielen der Aufhebung der Radwegebenutzungspflicht im HauptstraBennetz vorgesehen. Wären Sie bereit an einer weitergehenden 2. Befragung (telefonisch) teilzunehmen, um konkrete Untersuchungsbeispiele in Ihrer Kommune zu identifizieren, für welche detaillierte Unfalluntersuchungen sowie Begleituntersuchungen im Rahmen dieses Forschungsvorhabens von uns durchgeführt werden können?

Abschließend wurde im Fragebogen die Bereitschaft zu einer weitergehenden zweiten Befragung im Rahmen des Projektes geprüft. Diese Bereitschaft ist Grundvoraussetzung für die Identifizierung von potentiellen Untersuchungsstrecken der vorgesehenen Vorher-Nachher-Unfalluntersuchungen sowie der geplanten Begleituntersuchungen zum Verkehrsverhalten an künftig nicht mehr benutzungspflichtigen Radwegen.

Von den 231 antwortenden Städten sprachen sich $52 \%$ für eine weitere Befragung und $48 \%$ dagegen aus. Schlussendlich waren es insgesamt 119 Städte, die einer weiteren Befragung zur Identifizierung von konkreten Umsetzungsbeispielen zur Aufhebung der Radwegebenutzungspflicht offen gegenüberstanden. Dementsprechend zeigte sich für die hier weiter vorgesehenen Projektschritte auch ein erhebliches Untersuchungspotential.

Die finale Auswahl der potentiellen Untersuchungsstädte für die weiteren Untersuchungsschritte des vorliegenden Projektes (Vorher-Nachher-Unfallanalyse sowie Verkehrsverhaltensuntersuchung) wird in Kapitel 3.2 vorgenommen. 


\subsubsection{Zusammenfassung der Onlineumfrage}

Im Rahmen einer Onlineumfrage wurden die Erfahrungen von kommunalen Entscheidungsträgern zur Aufhebung der Radwegebenutzungspflicht zusammengetragen. Da es sich bei den Antworten der Onlineumfrage vorrangig um subjektive Einschätzungen der Befragten zur generellen Situation um die Radwegebenutzungspflicht und nicht zu einzelnen Radverkehrsanlagen handelt, sollten die hier zusammengefassten Erfahrungen als Hinweise verstanden werden. Insgesamt zeigt die Umfrage in den 679 deutschen Städten mit mehr als 20.000 Einwohnern mit einer Rücklaufquote von 52,4 \% ein erhöhtes Interesse der Städte an der Thematik der Radwegebenutzungspflicht.

Die Aufhebung der Radwegebenutzungspflicht halten $62 \%$ der 354 antwortenden Kommunen für sinnvoll. In Städten mit mehr als 50.000 Einwohnern wird die Aufhebung der Radwegebenutzungspflicht häufiger für sinnvoll gehalten als in kleinen Mittelstädten. In insgesamt $82 \%$ von 329 antwortenden Städten existieren baulich angelegte, straßenbegleitende Radwege ohne Benutzungspflicht. Die vor der Aufhebung erforderlichen Überprüfungen der Notwendigkeit der Benutzungspflicht an den Radwegen wurden in $42 \%$ von 268 Städten bereits begonnen oder zu $38 \%$ schon abgeschlossen. Wenn derartige Überprüfungen in den Städten stattfinden bzw. -fanden, dann sind diese Maßnahmen zu 70 \% (155 von 221 Städten) eigeninitiiert aufgrund von Änderungen der rechtlichen Rahmenbedingungen. Basierend auf den Überprüfungen wurde die Benutzungspflicht in den Städten auch in unterschiedlichem Umfang aufgehoben. Strukturierte Konzepte oder Strategien zum Vorgehen bei Aufhebung der Radwegebenutzungspflicht existieren hierbei nur in jeder fünften der 264 antwortenden Städte. Neben den bereits vorhandenen nicht benutzungspflichtigen Radwegen wird in Zukunft in 87 von 262 Städten (33\%) die Benutzungspflicht an (weiteren) Radwegen aufgehoben und dies auch überwiegend in größerem Umfang (mehr als 5 Radwege je Stadt). Insgesamt zeigt sich, dass in den Städten mit der Radweggebenutzungspflicht sehr unterschiedlich umgegangen wird, dies betrifft vorrangig die Überprüfungen der Radwegebenutzungspflicht als auch die Aufhebung selbst.

Bei den Begleitmaßnahmen zeigt sich eine sehr große Bandbreite angewandter Maßnahmen bei Aufhebung der Radwegbenutzungspflicht und eher keine einheitlichen Vorgehensweisen. Aus den Antworten kann aber nicht auf den Umfang von bestimmten Begleitmaßnahmen im Radwegenetz einer Stadt geschlussfolgert werden, stattdessen zeigen die Ergebnisse, dass die genannten Maßnahmen schon mindestens einmal umgesetzt worden sind. Sofern Begleitmaßnahmen umgesetzt werden, so handelt es sich vorrangig um die Markierung von Schutz- oder Radfahrstreifen. Weiterhin werden sehr häufig technische Anpassungen an signalgeregelten Knotenpunkten genannt., da sich durch die Radverkehrsführung im Mischverkehr die Zwischenzeiten verändern können. Neben diesen infrastrukturellen Maßnahmen werden auch häufig Informationskampagnen für die Verkehrsteilnehmer umgesetzt. Insgesamt wurden diese 3 Maßnahmen von den meisten befragten Städten schon mal bei der Aufhebung der Benutzungspflicht an Radwegen umgesetzt. In geringerem Umfang werden auch Geschwindigkeitsbeschränkungen im Kraftfahrzeugverkehr angeordnet, Radwege zurückgebaut oder Sperren oder Baken angebracht. Darüber hinaus erfolgt in Einzelfällen die Anordnung Gehweg Radfahrer frei, das Anbringen von nichtamtlichen Hinweisschildern sowie die Markierung von Piktogrammen auf der Fahrbahn oder dem verbleibenden nicht benutzungspflichtigen Radweg.

Grundlegend werden die genannten Begleitmaßnahmen basierend auf Einzelfallentscheidungen umgesetzt und überwiegend auch als zwingend notwendig angesehen. Standardmäßige Begleitmaßnahmen existieren hierbei nicht, bei den Entscheidungen sind stattdessen die individuellen Eigenschaften der Strecke von Belang. 
Unterhaltungsmaßnahmen werden an nicht benutzungspflichtigen Radwegen tendenziell seltener durchgeführt als an benutzungspflichtigen Radwegen. Unterhaltung- und Verkehrssicherungsmaßnahmen liegen dabei auf dem höchsten Niveau und weisen auch die geringsten Unterschiede zwischen Radwegen mit bzw. ohne Benutzungspflicht auf. Die größten Differenzen zwischen Radwegen mit bzw. ohne Benutzungspflicht zeigen sich bei Reinigungs- und Winterdiensten. Die Einflüsse veränderter Baulastträgerschaft mit Aufhebung der Radwegebenutzungspflicht auf die Ergebnisse sind dabei jedoch unklar.

Die subjektiven Einschätzungen der befragten Städte zu Auswirkungen der Aufhebung der Radwegebenutzungspflicht auf die Konflikthäufigkeit und Verkehrssicherheit zeigen, dass städtische Verantwortliche geringfügige Verbesserungen der Sicherheitsaspekte erwarten. Die anschließend abgefragten positiven und negativen Wirkungen zeigen jedoch auch sehr gegensätzliche Einschätzungen. Als positive Effekte der aufgehobenen Radwegebenutzungspflicht werden vorrangig Erhöhungen der Verkehrssicherheit, Verbesserungen des Fahrkomforts und der Sichtbarkeit von Radfahrern sowie angepasste Fahrweisen von Kraftfahrzeugführern erwartet. Zu den negativen Effekten zählen vorrangig Akzeptanzprobleme der Kraftfahrzeugführer gegenüber den Radfahrern auf der Fahrbahn sowie die generell geringe Fahrbahnnutzung durch die Radfahrer aufgrund von Regelunkenntnis und des subjektiven Unsicherheitsgefühls. Zudem wird auch von einer Zunahme der Konflikte auf der Fahrbahn oder durch die mögliche doppelte Radverkehrsführung sowie einer Verringerung der Verkehrsqualität ausgegangen. Insgesamt gehen aber auch $17 \%$ der Städte von ausbleibenden positiven oder negativen Effekten aus. Innerhalb der Verwaltungen werden weiterhin fehlende Handlungsempfehlungen, Mehrkosten, finanzielle Doppelbelastungen und somit potentielle Vernachlässigungen der nicht benutzungspflichtigen Radwege als Probleme angesehen. Die negativen Erwartungen spiegeln sich auch in der Einschätzung des Umfangs der Fahrbahnnutzung durch den Radverkehr wider. Hier wird von keinen wesentlichen Verlagerungen des Radverkehrs auf die Fahrbahn ausgegangen. Ebenfalls gering wird die Akzeptanz der Radfahrer im Mischverkehr durch die Kraftfahrzeugführer eingeschätzt. Abschließend gibt es nur in wenigen Städten nicht benutzungspflichtige Radwege mit einer erhöhten Fahrbahnnutzung durch den Radverkehr.

Bezüglich der Anordnungskriterien der Radwegebenutzungspflicht bleibt festzuhalten, dass die besondere Gefahrenlage für Radfahrer von den Städten vorrangig über die Verkehrsstärke und die Verkehrszusammensetzung bewertet wird. Zusammen mit dem Unfallgeschehen, den Querschnittsbreiten und den zulässigen Höchstgeschwindigkeiten werden diese Kriterien in mindestens jeder dritten Stadt zur Beurteilung der Gefahrenlagen bei Anordnung der Radwegebenutzungspflicht herangezogen. Auch bei den Anordnungskriterien aufgrund der Verkehrsqualität treten Verkehrsstärken und Schwerverkehrsanteile hervor. Die für die besondere Gefahrenlage und die Verkehrsqualität genannten, konkreten Grenzwerte waren dabei teilweise sehr unterschiedlich (Orientierung vorrangig an vorhandenen Regelwerken wie ERA oder RASt) oder wurden nicht genau angegeben. Somit verweist auch die Vielzahl der unterschiedlichen Kriterien und Grenzwerte darauf, dass offensichtlich für die Anordnung der Radwegebenutzungspflicht keine einheitlichen Kriterien verwendet werden. Zum Teil findet neben den - in den Regelwerken enthaltenen Richtwerten auch eine „erfahrungsbasierte Anordnung“ der Radwegebenutzungspflicht innerhalb der befragten Städte statt. 


\subsection{Städteauswahl für die weitergehende Untersuchung}

Aus den 119 Städten der Onlineumfrage, welche die Bereitschaft für eine weitergehende, detailliertere Befragung erklärten, galt es für die weiteren Untersuchungsschritte geeignete Städte zu identifizieren und hier entsprechend eine Auswahl zu treffen. Für diese Auswahl wurde eine Verschneidung verschiedener Befragungsergebnisse sowie weiterer Kriterien herangezogen. Diese lauten:

- in der Stadt werden möglichste viele verschiedene Begleitmaßnahmen bei Aufhebung der Radwegebenutzungspflicht umgesetzt,

- in der Stadt wird künftig an möglichst vielen Radwegen die Benutzungspflicht aufgehoben,

- die ausgewählten Städte weisen eine angemessene Verteilung auf das Bundesgebiet und

- ein ausreichend hohes Radverkehrsaufkommen auf (gemessen am Modal Split gemäß SrV [Ahrens et al 2015], [EPOMM 2015]).

Die Ergebnisse des aktuellen Fahrradklimatestes wurden nur prüfend herangezogen ${ }^{2}$. Für die ausgewählten Städte wurde geprüft, dass nicht nur Städte mit gutem Fahrradklima, hohem Stellenwert des Radverkehrs oder hoher Verkehrssicherheit ausgewählt wurden, sondern sich auch hier eine ausgewogene Verteilung ergibt. Entsprechend der genannten Kriterien wurden insgesamt 19 Städte für die weiteren Untersuchungsschritte sowie die anschließende zweite Umfragephase ausgewählt. Dabei ergab sich ebenfalls ein ausgewogenes Verhältnis verschiedener Städtegrößen. Die notwendige Clusterung von Radwegen ohne Benutzungspflicht sowie das weitere Vorgehen zur Telefonbefragung werden in den nachfolgenden Kapiteln näher erläutert.

\subsection{Definition von Typen nicht benutzungspflichtiger Radwege}

Für die weiteren Untersuchungen wurden Cluster verschiedener Typen von nicht benutzungspflichtigen Radwegen definiert. Das wesentliche Merkmal zur Definition verschiedener Typen bzw. Charakteristiken von nicht benutzungspflichtigen Radwegen stellen dabei die unterschiedlichen Begleitmaßnahmen bei Aufhebung der Radwegenutzungspflicht dar. Die nachfolgende Clusterung erfolgte somit entsprechend der in Kapitel 3.1.2.2 identifizierten Begleitmaßnahmen bei Aufhebung der Radwegebenutzungspflicht unter Berücksichtigung deren Häufigkeit.

Entsprechend Tabelle 3 besteht Cluster 1 für die weitere Untersuchung aus Radwegen mit aufgehobener Benutzungspflicht, an denen parallel zur Aufhebung keine weiteren flankierenden Maßnahmen umgesetzt wurden. Die im Rahmen der Onlineumfrage gemeinsame Antwortmöglichkeit „Markierung Radfahrstreifen/Schutzstreifen“ wurde für die weiteren Schritte getrennt, da ein Radfahrstreifen (Cluster 2) grundlegend nur eine Verlagerung der Benutzungspflicht auf die Fahrbahn darstellt und es sich bei einem Schutzstreifen (Cluster 3) dagegen nicht um eine verkehrsbehördliche Anordnung einer Benutzungspflicht handelt. Cluster 4 beinhaltet zudem nicht benutzungspflichtige Radwege, bei denen parallel zur Aufhebung Geschwindigkeitsbeschränkungen für den Kraftfahrzeugverkehr angeordnet wurden. Cluster 5 enthält sowohl den Rückbau der Radwege als auch das Anbringen von Sperren/Baken als Begleitmaßnahme, da hier nach Kapitel 3.1.2.2 in beiden Fällen kein Radweg mit Benutzungsrecht mehr vorhanden ist. Die verbleibenden maßgebenden Begleitmaßnahmen sind die Anordnung Gehweg Radfahrer frei (Cluster 6), das Anbringen nichtamtlicher Hinweisschilder (Cluster 7) sowie das Markieren von Piktogrammen (Cluster 8).

\footnotetext{
${ }^{2}$ Der Fahrradklimatest fasst 27 Einzelfragen zu 5 Kategorien (Fahrrad- und Verkehrsklima, Stellenwert des Radverkehrs, Sicherheit beim Radfahren, Komfort beim Radfahren, Infrastruktur Radverkehrsnetz) zusammen, wobei die Ergebnisse der 5 Kategorien untereinander stark korrelieren [Böhmer 2015].
} 


\begin{tabular}{|l|l|}
\hline $\begin{array}{l}\text { Begleitmaßnahme bei Aufhebung der } \\
\text { Radwegebenutungspflicht }\end{array}$ & Clusterdefinition und Bemerkungen \\
\hline keine Begleitmaßnahmen & Cluster 1 \\
\hline Markierung Radfahrstreifen & Cluster 2 \\
\hline Markierung Schutzstreifen & Cluster 3 \\
\hline Geschwindigkeitsbeschränkung Kraftfahrzeugverkehr & Cluster 4 \\
\hline Rückbau Radwege & Cluster 5 \\
\hline Sperren/Baken & zu Cluster 5 \\
\hline Gehweg "Radfahrer frei" & Cluster 6 \\
\hline Nichtamtliche Hinweisschilder & Cluster 7 \\
\hline Markierung von Piktogrammen & Cluster 8 \\
\hline Signaltechnische Anpassungen & entfällt, da Pflicht zur Prüfung (keine Wahlmaßnahme) \\
\hline Informationskampagnen & entfällt, da informative Maßnahme unterschiedlicher Tiefe \\
\hline Zulassen von Parken & entfält, da Einzelfall \\
\hline Öffnung Einbahnstraße & entfällt, da Einzelfall \\
\hline Freigabe linksseitiger Radwege & entfällt, da Einzelfall \\
\hline Markierung Aufstellflächen an KP & entfält, da Einzelfall \\
\hline Umwidmung Fahrradstraße & entfällt, da Einzelfall \\
\hline
\end{tabular}

Tabelle 3: Clusterung von nicht benutzungspflichtigen Radwegen

Signaltechnische Anpassungen wurden nicht als eigenes Cluster für die vorliegende Untersuchung ausgewählt, da diese Maßnahmen in der Regel in Kombination mit einer der jeweils anderen Maßnahmen durchgeführt werden. Weiterhin gehören Prüfungen und notwendig werdende Anpassungen der Zwischenzeiten bzw. der Signalisierung zum Standardprozedere bei Aufhebung der Radwegebenutzungspflicht, da nach RiLSA [2010] bei Mischverkehrsführung auf längere Räumzeiten zu achten ist.

Weiterhin wurden Informationskampagnen nicht näher berücksichtigt, da es sich hierbei um informative Maßnahmen sehr unterschiedlicher Tiefe und Wirksamkeit handelt, jedoch nicht um infrastrukturelle/betriebliche Maßnahmen. Zudem war die Bewertung von Informationskampagnen mit den hier vorgesehenen Methoden der Unfall- und Verkehrsverhaltensanalyse nicht möglich. Auch alle anderen in Kapitel 3.1.2.2 identifizierten Begleitmaßnahmen wurden nicht für die weiteren Untersuchungen herangezogen, da es sich bei diesen Maßnahmen um sehr spezielle Begleitmaßnahmen (Einzelfälle) an nicht benutzungspflichtigen Radwegen handelt.

\subsection{Zweite Umfragephase (Telefonbefragung)}

Für die in Kapitel 3.2 ausgewählten Städte wurde eine zweite telefonische Befragung zu konkreten Umsetzungsbeispielen der Aufhebung der Radwegebenutzungspflicht durchgeführt. Das Ziel der weitergehenden Befragung war die Identifizierung von insgesamt 100 Radwegen ohne Benutzungspflicht im Hauptstraßennetz der Städte, für die Vorher-Nachher-Unfalluntersuchungen im Rahmen des Projektes durchgeführt werden konnten. Weiterhin galt es 10 Radwege verschiedener Charakteristik für eine potentielle Vorher-Nachher-Verkehrsverhaltensanalyse zu identifizieren, an denen während der Projektlaufzeit die Radwegebenutzungspflicht aufgehoben wurde und für die somit eine Begleituntersuchung durchgeführt werden konnte.

Im Rahmen der telefonischen Befragung wurden neben allgemeinen Fragen zur Radwegebenutzungspflicht die weiteren Projektziele detailliert erläutert und die interessierenden Informationen für die auszuwählenden Untersuchungsstrecken diskutiert. Im Nachgang zum Telefongespräch sollten seitens der Städte nach Möglichkeit insgesamt 16 Untersuchungsbeispiele mit bereits aufgehobener Radwegebenutzungspflicht genannt und hierfür weitere gewünschte Informationen in einer Tabelle zusammengetragen werden. Die potentiellen Untersuchungsstrecken sollten hierbei die folgenden Eigenschaften aufweisen: 
- $\quad$ nicht mehr benutzungspflichtiger Radweg im Hauptstraßennetz mit hohem Kfz- und Radverkehrsaufkommen

- Aufhebung der Radwegebenutzungspflicht vor dem Jahr 2014

- vor Aufhebung der Benutzungspflicht handelte es sich um einen separaten baulichen Radweg gemäß den Zeichen 237 oder 241 StVO

- Länge des Radweges von ca. 500 m bis 1.000 m idealerweise mit gleicher Charakteristik

- die nicht benutzungspflichtigen Radwege entsprechen einem der 8 definierten Cluster (vgl. Kapitel 3.3) von den in Städten üblicherweise angewendeten Begleitmaßnahmen

Als zusätzliche Informationen für die nicht benutzungspflichtigen Radwege wurden abgefragt:

- genauer Zeitpunkt der Aufhebung der Radwegebenutzungspflicht

- zulässige Höchstgeschwindigkeit vor Aufhebung der Radwegebenutzungspflicht

- Grund der Aufhebung der Radwegebenutzungspflicht

- positive/negative Erfahrungen am konkreten Beispiel

- Zustand des nicht benutzungspflichtigen Radweges

- Parkdruck auf dem Streckenabschnitt

- Höhe der Verkehrsbelastungen für Kfz-/Rad- und Fußgängerverkehr

- Anteil der Radfahrer auf der Fahrbahn neben dem nicht benutzungspflichtigen Radweg

Im Nachgang zum Telefongespräch sollten von den einzelnen Städten weiterhin jeweils bis zu 10 Untersuchungsbespiele genannt werden, an denen die Radwegebenutzungspflicht noch aufgehoben wird. Für diese Radwege mit noch vorhandener Benutzungspflicht galten die gleichen oben genannten Randbedingungen wie für Radwege mit bereits aufgehobener Benutzungspflicht. Grundvoraussetzung für die Betrachtung eines solchen Radweges im Rahmen des Projektes war die vorgesehene Aufhebung der Radwegebenutzungspflicht im Jahr 2016 bzw. 2017, sodass Vorher-Nachher-Verkehrsverhaltensuntersuchungen während der Laufzeit des vorliegenden Projektes möglich waren. Zur abschließenden Auswahl von insgesamt 10 - für entsprechende Begleituntersuchungen geeigneten - Radwegen unterschiedlicher Charakteristik wurden ebenfalls zusätzliche Informationen abgefragt, welche den oben genannten Zusatzinformationen entsprechen.

\subsection{Ergebnisse der Telefonbefragung}

Nach Erhalt aller Rückmeldungen der Städte konnten die Ergebnisse in einer Datenbank mit potentiellen Untersuchungsstrecken zusammengeführt werden. Im Rahmen der makroskopischen Unfallanalyse fand dann aus der Gesamtheit der genannten Beispiele eine Auswahl von 100 näher zu untersuchenden Radwegen ohne Benutzungspflicht statt. Weiterhin galt es 10 geeignete Radwege für die Vorher-Nachher-Verkehrsverhaltensanalysen aus den genannten Beispielen auszuwählen. Das nachfolgende Kapitel enthält die Beschreibung der Vorgehensweisen der entsprechend folgenden Arbeitsschritte. 


\section{Methodik der Unfall- und Verkehrsverhaltensuntersuchung}

Dieses Kapitel enthält die Vorgehensweise der detaillierten Untersuchungen zum Unfallgeschehen und Verkehrsverhalten mit Aufhebung der Radwegebenutzungspflicht basierend auf den Erkenntnissen der Literaturanalyse (Kapitel 2) und den Ergebnissen beider Befragungsphasen (Kapitel 3).

\subsection{Makroskopische Unfallanalyse}

Ziel der makroskopischen Unfallanalyse war die Bewertung der Veränderungen im Unfallgeschehen mit Aufhebung der Radwegebenutzungspflicht. Hierzu wurde eingangs die allgemeine Unfallentwicklung (Unfälle mit/ohne Radverkehrsbeteiligung) an ausgewählten Radwegen differenziert nach knotenpunktfreien Streckenabschnitten und Knotenpunkten vor und nach Aufhebung der Radwegebenutzungspflicht betrachtet. Zur Validierung dieser Ergebnisse wurden weiterhin die zeitlichen Entwicklungen im Unfallgeschehen (Anteil der Unfälle mit Radverkehrsbeteiligung an allen Unfällen) an den ausgewählten Radwegen sowie die zeitliche Unfallentwicklung der Gesamtstadt prüfend gegenübergestellt. Anschließend erfolgte die Berechnung von Unfallkenngrößen differenziert nach ausgewählten infrastrukturellen Merkmalen sowie die Auswertung der makroskopischen Unfallmerkmale. Zur Erklärung relevanter Veränderungen im Unfallkollektiv wurden hier später weitere infrastrukturelle Radwegeigenschaften herangezogen. Durch die makroskopische Unfallanalyse waren insgesamt Hinweise auf besonders auffällige Verkehrssituationen und Rückschlüsse darauf, welche verkehrlichen und entwurfstechnischen Merkmale mit Aufhebung der Radwegebenutzungspflicht ggf. ein besonders hohes Unfallrisiko hervorrufen, zu generieren.

\subsubsection{Auswahl von Untersuchungsstrecken}

Wesentliche Grundlage der nachfolgenden Unfallanalysen waren die nach der Telefonbefragung (vgl. Kapitel 3.4) von 10 Städten bereitgestellten Informationen zu 146 Streckenzügen mit insgesamt 212 Radwegen ohne Benutzungspflicht. Für die Strecken erfolgte eine Prüfung der Eignung der betreffenden Radwege für die vorliegende Untersuchung. Grundlegend wurden bei entsprechender Eignung auch die Radwege auf beiden Seiten eines Streckenzuges verwendet. Im Zuge der Streckenprüfung wurden Zweirichtungsradwege und gemeinsame Geh- und Radwege - welche nicht Bestandteil der vorliegenden Untersuchung sind - sowie weitere Radwege ausgeschlossen, deren Zeitpunkt der Aufhebung der Radwegebenutzungspflicht nicht innerhalb des Zeitraumes verfügbarer Unfalldaten lag. Radwege, an denen neben dem Jahr der Aufhebung der Radwegebenutzungspflicht auch im Vorher-Nachher-Zeitraum umfassende Baumaßnahmen stattfanden, wurden ebenfalls vernachlässigt. Im Untersuchungskollektiv verblieben abschließend 108 nicht benutzungspflichtige Radwege für die weiteren Bearbeitungsschritte. Diese Radwege haben eine Gesamtlänge von 84,1 Kilometern und befinden sich in den folgenden 8 Städten:

- Berlin

- Bremen

- Düsseldorf

- Halle (Saale)
- Hamburg

- Nürnberg

- Paderborn

- UIm

Eine Übersicht der betrachteten Radwege nach Begleitmaßnahmen und Städten enthält Tabelle 4. Es wird deutlich, dass Radwege ohne weitere Begleitmaßnahmen im untersuchten Kollektiv mit $70 \%$ am stärksten vertreten sind. Zudem blieben 4 Cluster nach Kapitel 3.3 unbesetzt. Die wenigen ursprünglich genannten Radwege in 3 dieser Cluster (2 zurückgebaute Radwege an denen benutzungspflichtige Radfahrstreifen angelegt wurden, 4 Radwege mit Beschränkung der zulässi- 
gen Höchstgeschwindigkeit sowie 3 Radwege mit Anordnung nichtamtlicher Hinweisschilder nach Aufhebung der Radwegebenutzungspflicht) konnten nach erfolgter Streckenprüfung aufgrund fehlender Eignung nicht weiter berücksichtigt werden. Die Beschränkung der zulässigen Höchstgeschwindigkeit wurde in der Onlineumfrage (vgl. Kapitel 3.1.2.2) als mögliche Begleitmaßnahme vergleichsweise häufig genannt, jedoch gab dies keine Hinweise auf den tatsächlichen Umfang dieser Maßnahme insgesamt und war in den Streckennennungen der Städte als Einzelmaßnahme nur sehr selten enthalten. Nicht amtliche Hinweisschilder waren dagegen schon in der Onlineumfrage eine der selteneren Begleitmaßnahmen. Auch die Anlage eines benutzungspflichtigen Radfahrstreifens galt in Hinblick auf die Radwegebenutzungspflicht hier bereits generell als Sonderfall.

Weiterhin zeigte sich, dass der Rückbau von Radwegen nur in Kombination mit der Markierung von Schutzstreifen, dagegen nie als alleinige Maßnahme, auftrat. Zudem ergaben sich Überlappungen zwischen der Markierung von Schutzstreifen und Radfahrerpiktogrammen. Um derartige Dopplungen zu vermeiden, wurden maßgebende Begleitmaßnahmen - in den beiden beschriebenen Fällen die Schutzstreifen - definiert. Die Übersicht nach Tabelle 4 enthält nur Radwege ohne entsprechende Überschneidungen.

\begin{tabular}{|c|c|c|c|c|c|c|c|c|c|c|c|}
\hline \multirow[t]{2}{*}{$\begin{array}{l}\frac{\pi}{0} \\
\text { in } \\
0\end{array}$} & \multirow{2}{*}{ 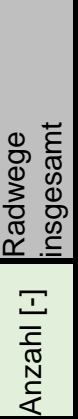 } & \multicolumn{2}{|c|}{ 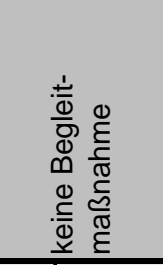 } & \multicolumn{2}{|c|}{ 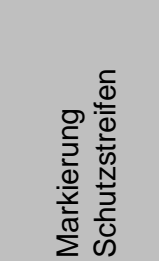 } & \multicolumn{2}{|c|}{ 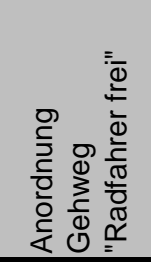 } & \multicolumn{2}{|c|}{ 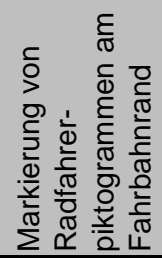 } & \multicolumn{2}{|c|}{ 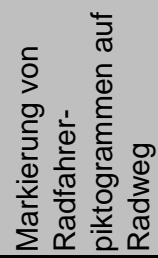 } \\
\hline & & 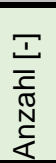 & 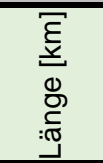 & 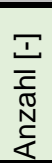 & 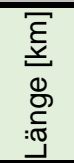 & 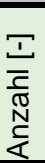 & 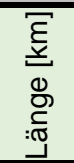 & $\begin{array}{l} \\
\\
\frac{1}{\mathbb{N}} \\
N \\
\frac{1}{4} \\
\end{array}$ & 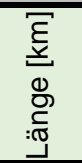 & $\begin{array}{l} \\
\\
\frac{1}{\mathbb{N}} \\
\stackrel{N}{<} \\
\frac{5}{4}\end{array}$ & 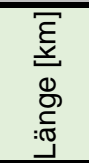 \\
\hline Berlin & 27 & 23 & 21,26 & & & 4 & 2,71 & & & & \\
\hline Bremen & 6 & 4 & 5,85 & & & & & 2 & 1,35 & & \\
\hline Düsseldorf & 12 & 10 & 5,15 & 1 & 0,56 & 1 & 0,89 & & & & \\
\hline Halle (Saale) & 21 & 19 & 10,87 & 1 & 0,19 & 1 & 0,54 & & & & \\
\hline Hamburg & 22 & 17 & 12,42 & 4 & 4,08 & 1 & 1,49 & & & & \\
\hline Nürnberg & 10 & 2 & 2,98 & & & & & & & 8 & 4,19 \\
\hline Paderborn & 8 & & & 3 & 2,32 & & & & & 5 & 6,12 \\
\hline Ulm & 2 & 1 & 0,56 & 1 & 0,56 & & & & & & \\
\hline Summe & 108 & 76 & 59,09 & 10 & 7,71 & 7 & 5,63 & 2 & 1,35 & 13 & 10,31 \\
\hline
\end{tabular}

Tabelle 4: Ausgewählte Radwege ohne Benutzungspflicht nach Städten und Begleitmaßnahmen

\subsubsection{Merkmalserhebung und Datenbankerstellung}

Für die identifizierten Radwege erfolgte eine eindeutige Codierung und Digitalisierung in einem Geoinformationssystem. Dabei wurden die Fahrbahnen sowie die angrenzenden nicht benutzungspflichtigen Radwege seitengetrennt aufgenommen. Weiterhin wurden zur Differenzierung des Unfallgeschehens von knotenpunktfreien Streckenabschnitten und Knotenpunkten folgende Definition von Knotenpunktbereichen herangezogen und diese entsprechend implementiert:

- $\quad$ signalisierte Knotenpunkte:

o Zufahrten: $25 \mathrm{~m}$ vor Haltelinie oder Anfang der Verziehung von

Rechts- bzw. Linksabbiegefahrstreifen

o Ausfahrten: $5 \mathrm{~m}$ nach Ende der kreuzenden Furt

- nicht signalisierte Knotenpunkte:

o Zufahrten: $25 \mathrm{~m}$ vor Fahrbahnrand der kreuzenden Straße

o Ausfahrten: $10 \mathrm{~m}$ nach Fahrbahnrand der gekreuzten Straße 
Insgesamt wurden mit dieser Definition 594 Knotenpunktbereiche innerhalb der untersuchten 108 Radwege angelegt.

Für die definierten Bereiche der knotenpunktfreien Strecke und Knotenpunkte wurden infrastrukturelle und betriebliche Merkmale durch Datenanfragen in den Kommunen (u.a. Art der Begleitmaßnahme, Datum der Aufhebung der Benutzungspflicht, Verkehrsbelastungen des Kraftfahrzeugund Radverkehrs) und Luftbildauswertungen sowie Vor-Ort-Besichtigungen (u.a. Breite der Querschnittselemente, Anlagen des ruhenden Verkehrs auf knotenpunktfreier Strecke sowie Vorfahrtsregelung und Anzahl von Abbiegefahrstreifen an Knotenpunkten) ermittelt. Während der Merkmalserhebung erfolgte zudem eine Prüfung der Homogenität der Merkmale am ausgewählten Radweg. Waren hier wesentliche Merkmalsunterschiede innerhalb längerer Streckenzüge erkennbar, erfolgte eine Teilung des untersuchten Radweges an entsprechender Stelle. Tabelle 4 enthält das Endergebnis dieser Aufbereitung. Abschließend wurden die infrastrukturellen Merkmale auf Änderungen im Zeitverlauf vor bzw. nach Aufhebung der Radwegebenutzungspflicht über historische Luftbilder geprüft. Da es sich vorrangig um betriebliche Maßnahmen bei Aufhebung der Radwegebenutzungspflicht handelt (Abnehmen der Beschilderung ohne weitere Begleitmaßnahmen) nach Tabelle 4 waren derartige Änderungen jedoch nur selten festzustellen.

Die für die späteren Auswertungen notwendigen Verkehrsstärken des Kraftfahrzeug- und Radverkehrs konnten seitens der Städte nicht für alle Radwege bereitgestellt werden und wurden ergänzt durch eigene zweistündige Kurzzeitzählungen an den Straßenquerschnitten. Bei den Radverkehrszählungen wurde dabei deren Flächennutzung vermerkt, wodurch Aussagen zur Akzeptanz des Fahrbahnfahrens der Radfahrer nach Aufhebung der Radwegebenutzungspflicht generiert werden konnten. Anschließend erfolgte eine Hochrechnung der gezählten Fahrzeugstärken. Die Zählungen des Kraftfahrzeugverkehrs unter 3,5 t wurden dabei anhand der Ganglinien für Straße-Umfeld-Typen nach Arnold et al. [2008] auf Tageswerte sowie abgeleitete Tag-/Wocheund Halbmonatsfaktoren aus Arnold et al. [2008] und HBS [2001] auf die durchschnittliche Jahresbelastung hochgerechnet. Für den Schwerverkehr wurden zur Hochrechnung die Lkw-Ganglinien und Tag-Wochen-Faktoren nach Schmidt et al. [1996] sowie die Halbmonatsfaktoren nach HBS [2001] verwendet. Die Hochrechnungen der Zählungen im Radverkehr erfolgten entsprechend dem „Hochrechnungsmodell von Stichprobenzählungen für den Radverkehr“ [Schiller et al. 2011].

\subsubsection{Unfallzuordnung und Datenbankerstellung}

Nach Erhalt der angefragten Unfalldaten aus den betrachteten 8 Städten erfolgte eine Aufbereitung der Unfalldatenbanken in Hinblick auf eine einheitliche Datenbankstruktur. Da in den 8 Städten nur Unfalldaten unterschiedlicher Unfallkategorien verfügbar waren, beschränkte sich die Unfallanalyse zur Wahrung der Vergleichbarkeit generell auf die Unfälle mit Personenschaden (Unfallkategorien 1-3) als Schnittmenge aller verfügbarer Unfalldaten. Für die georeferenzierten Unfälle erfolgte anschließend im Geoinformationssystem eine örtliche Zuordnung zu den erstellten Strecken- und Knotenpunktbereichen. Dadurch erfolgte eine Verknüpfung der eindeutigen Strecken- und Unfallcodierung zur abschließenden Erstellung einer Gesamtdatenbank mit allen relevanten infrastrukturellen Radweg- und Straßeneigenschaften sowie Unfallmerkmalen.

Im nächsten Schritt der Datenaufbereitung erfolgte eine zeitliche Zuordnung der Unfälle in Bezug auf den Zeitpunkt der Aufhebung der Radwegebenutzungspflicht. Hierfür wurde das Jahr der Aufhebung der Radwegebenutzungspflicht vom Unfalljahr subtrahiert und bei negativem Ergebnis der Vorher-Zeitraum und entsprechend positiven Ergebnis der Nachher-Zeitraum definiert (vgl. Schema nach Abbildung 23). Unfälle im Jahr der Aufhebung wurde dagegen nicht betrachtet. 


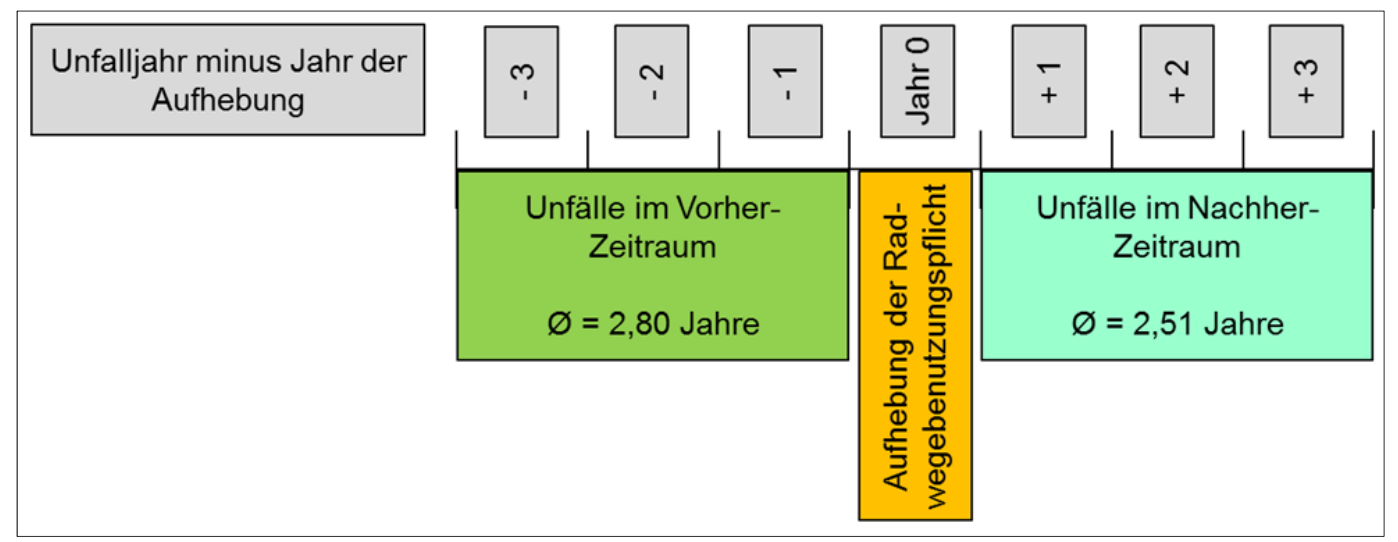

\section{Abbildung 23: Herstellung des Zeitbezuges zwischen Unfalldatum und Zeitpunkt der Aufhebung der Radwegebenutzungspflicht}

Als Ergebnis der Unfallzuordnung wurden den 108 betrachteten Radwegen und angrenzenden Fahrstreifen der entsprechenden Fahrtrichtung insgesamt 2.392 Unfälle mit Personenschaden und davon 890 Unfälle mit Radverkehrsbeteiligung zugewiesen.

In Abhängigkeit des Zeitpunktes der Aufhebung der Radwegebenutzungspflicht und der entsprechend in den Städten verfügbaren Unfalldaten umfassten die Zeiträume mit auswertbarem Unfallgeschehen vor und nach Aufhebung der Radwegebenutzungspflicht für mehrere der 108 betrachteten Radwege jedoch eine unterschiedliche Anzahl an Jahren. Davon betroffen waren ältere aber auch vorrangig jüngere Aufhebungen der Radwegebenutzungspflicht ab dem Jahr 2013, da hier zum Zeitpunkt der Projektbearbeitung noch nicht auf einen vollen 3 Jahreszeitraum nach Aufhebung der Radwegebenutzungspflicht zurückgegriffen werden konnte. An $36 \%$ der betrachteten Radwege wurde die Benutzungspflicht erst ab dem Jahr 2013 aufgeboben (insgesamt an 89 \% der betrachteten Radwege nach dem Jahr 2010, vgl. dazu Kapitel 2.1.3). Um hier nicht weitere Radwege aus dem Untersuchungskollektiv wegen eines notwendigen 3-jährigen Zeitraumes vor und nach Aufhebung der Radwegebenutzungspflicht entfernen zu müssen, wurde das Minimum des Untersuchungszeitraumes auf 1 Jahr und das Maximum auf 3 Jahre festgelegt. Aufgrund der möglichen unterschiedlichen Anzahl an Jahren verfügbarer Unfalldaten in den Zeiträumen vor und nach Aufhebung der Radwegebenutzungspflicht erfolgte eine Normierung der Unfälle auf Unfälle pro Jahr. Somit wird in allen folgenden Auswertungen zur Ermöglichung des Vorher-NachherVergleiches eine Bewertung der Unfälle pro Jahr vorgenommen.

\subsubsection{Sensitivitätsanalyse des Untersuchungskollektives}

Zur Bewertung der Ergebnisse wurde die Unfallentwicklung der einzelnen betrachteten Radwege im Untersuchungskollektiv zudem über die Jahre hinweg betrachtet und mit der Unfallentwicklung der Gesamtstadt verglichen. Der Vergleich erfolgte dabei nach Einzeljahren und hier über den Quotienten aus Radunfällen mit Personenschaden und allen Unfällen mit Personenschaden. Die Unfalldaten der Gesamtstadt wurden sofern möglich aus vorliegenden Unfalldatenbanken sowie den jährlichen Veröffentlichungen der Städte zum Unfallgeschehen entnommen.

Durch diese Betrachtung sollten die ausgewählten Radwege in das Gesamtunfallgeschehen bzw. dessen -entwicklung innerhalb der Städte eingeordnet, somit das ausgewählte untersuchte Streckenkollektiv bewertet und hier mögliche Ausreißer identifiziert werden. Somit war es möglich, in den Auswertungen etwaige vorherrschende Trends im Unfallgeschehen über die Jahre zu berücksichtigen und ein Vergleichskollektiv zu dem ausgewählten Untersuchungskollektiv zur besseren Bewertung im Vorher-Nachher-Vergleich heranzuziehen. 


\subsubsection{Unfallkenngrößenberechnung}

Zur Bewertung der beschriebenen allgemeinen Unfallentwicklung im Radunfallgeschehen vor und nach Aufhebung der Radwegebenutzungspflicht wurden weiterhin Unfallkenngrößen für die wesentlichen infrastrukturellen Merkmale ermittelt. Deren Berechnung folgte dabei den Vorgaben für Straßenzüge gemäß dem Merkblatt für die Auswertung von Straßenverkehrsunfällen [FGSV 2003]. Vor Berechnung der auf Unfallkosten basierenden Kenngrößen erfolgte eine Anpassung der Unfallkostensätze für Radunfälle auf Basis des verwendeten Unfalldatensatzes. Das Ergebnis enthält Tabelle 5. Zum Vergleich sind auch die Kostensätze für Unfälle ohne Radverkehrsbeteiligung im untersuchten Kollektiv gegenübergestellt.

\begin{tabular}{|c|c|c|c|}
\cline { 3 - 4 } \multicolumn{2}{c|}{} & \multicolumn{2}{c|}{ Unfallkollektiv } \\
\cline { 3 - 4 } \multicolumn{2}{c|}{} & Unfall ohne Radbeteiligung & Radunfall \\
\hline $\begin{array}{c}\text { Unfall- } \\
\text { kategorie }\end{array}$ & $U(\mathrm{SP})$ & $154.572 €$ & $145.018 €$ \\
\cline { 2 - 4 } & $\mathrm{U}(\mathrm{LV})$ & $15.165 €$ & $14.250 €$ \\
\hline
\end{tabular}

Tabelle 5: Verwendete angepasste Unfallkostensätze (Verunglückten- und Sachschadenskostensätzen nach Unfallstruktur 2006-2010, Preisstand 2010)

\subsubsection{Berücksichtigung der Radverkehrsentwicklung}

Grundlegend kann zu der - im Rahmen der Sensitivitätsanalysen betrachteten - allgemeinen Entwicklung im Unfallgeschehen auch die Entwicklung des Radverkehrsaufkommens generell beigetragen haben. Hintergrund ist hierbei, dass sich bei einem anzunehmenden Wachstum des Radverkehrsaufkommens in den vergangenen Jahren auch entsprechende Veränderungen im Radunfallgeschehen eingestellt haben können. Diesem Aspekt sollte bei Berechnung der Unfallkenngrößen Rechnung getragen werden. Bei der Berechnung von Unfallraten und Unfallkostenraten wurde daher ein gestiegenes Radverkehrsaufkommen bei den Radverkehrsstärken als Expositionsgröße berücksichtigt, um Vergleiche vor und nach Aufhebung der Radwegebenutzungspflicht zu ermöglichen. Es war weiterhin zu beachten, dass in Abhängigkeit der Datenlage entweder Radverkehrsstärken vor oder nach Aufhebung der Radwegebenutzungspflicht zur Verfügung standen.

Um die angesprochenen Vergleiche zu ermöglichen, wurde eine vereinheitlichende Projektion der vorliegenden Radverkehrsstärken in das Jahr der Aufhebung der Radwegebenutzungspflicht vorgenommen. Dabei erfolgte für ältere verwendete Zählungen eine Hochrechnung und für die während des Projektes ermittelten Radverkehrsstärken eine Rückrechnung der Verkehrsstärken auf die Verkehrsstärken im Jahr der Aufhebung der Radwegebenutzungspflicht. Dabei wurde eine konstante jährliche Änderung des Radverkehrsaufkommens angenommen. Nach Projektion in das Jahr der Aufhebung der Radwegebenutzungspflicht erfolgte eine weitere Projektion zu den mittleren Verkehrsstärken der auswertbaren Vorher- und Nachherzeiträume (bei Vorliegen eines 3Jahreszeitraumes eine Projektion der Radverkehrsstärken von 1,5 Jahre vor bzw. nach Aufhebung der Radwegebenutzungspflicht, bei geringeren Zeiträumen entsprechend angepasst). Die jährliche Radverkehrsentwicklung der Städte wurde mit Hilfe der Berichte zur Mobilität in Städten (vgl. Ahrens et al. 2010 und Ahrens et al. 2015) abgeschätzt (Ergebnisse dieser Schätzung siehe Kapitel 5.3.1). In Hamburg wurde die Radverkehrsentwicklung aus Langzeitzähldaten der Stadt abgeleitet. Erst unter Berücksichtigung der Unfallentwicklung und der Radverkehrsentwicklung kann in diesem Projekt eine Bewertung der Ergebnisse erfolgen. 


\subsubsection{Bestandteile der makroskopischen Unfallanalyse}

Im Rahmen der makroskopischen Unfallanalyse wurden unter anderem die folgenden Unfallmerkmale im Radunfallgeschehen differenziert nach knotenpunktfreier Strecke und Knotenpunkten ausgewertet:

- Unfallkategorien

- Unfalltypen

- Unfallarten

- Straßen- und Lichtverhältnisse
- Hauptunfallverursacher

- Alter, Geschlecht, Art der Verkehrsbeteiligung Unfallursachen differenziert nach Hauptverursachern

Dabei erfolgte die makroskopische Unfallanalyse zunächst für Radwege ohne Benutzungspflicht ohne weitere Begleitmaßnahmen als größtes Cluster gemäß Tabelle 4. Die Erkenntnisse der Differenzierung nach den anderen Begleitmaßnahmen wurden dennoch bei besonderen Auffälligkeiten und Unterschieden berücksichtigt. Weiterhin war bei den Betrachtungen sämtlicher ausgewerteter Unfallmerkmale im Kontext der Aufhebung der Radwegebenutzungspflicht grundlegend die tatsächliche Flächennutzung des Radfahrers (Radfahrer im Seitenraum auf Rad- oder Gehwegen sowie Radfahrer auf der Fahrbahn) zu berücksichtigen, da hier die erlaubte Fahrbahnnutzung nach Aufhebung der Radwegebenutzungspflicht zu bestimmten Verlagerungen geführt haben kann. Die Ermittlung der hierfür notwendigen Informationen aus der mikroskopischen Unfallanalyse sowie die entsprechend notwendige, gemeinsame Auswertung der makroskopischen und mikroskopischen Unfalldaten wird in nachfolgendem Kapitel beschrieben.

\subsection{Mikroskopische Unfallanalyse}

Neben der makroskopischen Unfallanalyse verfolgte die mikroskopische Unfallanalyse das Ziel, weitere sicherheitsrelevante Aspekte der Unfälle mit Radverkehrsbeteiligung - vorrangig jedoch die Flächennutzung der beteiligten Radfahrer - zu identifizieren. Diese räumliche Unterscheidung nach dem Fahren im Straßenseitenraum (Fahren auf Radweg oder Gehweg) und dem Fahrbahnfahren stellt bei der Bewertung des Unfallgeschehens vor und nach Aufhebung der Radwegebenutzungspflicht eine bedeutsame Information dar. Erst nach der systematischen Auswertung der mikroskopischen Unfalldaten konnten die Auswirkungen der Aufhebung der Radwegebenutzungspflicht auf das Radunfallgeschehen abschließend bewertet werden, was allein auf makroskopischer Ebene nicht möglich war. Somit erfolgte die mikroskopische Unfallanalyse zur Erklärung der makroskopischen Unfalldaten und die Ergebnisdarstellung für beide Analyseebenen gemeinsam.

Aufgrund der fehlenden Verfügbarkeit mikroskopischer Unfalldaten konnte die mikroskopische Analyse jedoch nicht für alle 890 Radunfälle mit Personenschaden auf den untersuchten Radwegen durchgeführt werden. In drei Städten war daher die Analyse von mikroskopischen Unfalldaten generell nicht möglich. In weiteren zwei Städten erfolgte eine zusätzliche Einschränkung, da hier nur für Teile der Vorher-/Nachher-Zeiträume mikroskopische Unfalldaten zur Verfügung standen. Waren hier aufgrund älterer Aufhebungen der Radwegebenutzungspflicht nicht in beiden Zeiträumen gleichermaßen mikroskopische Unfalldaten verfügbar, so wurde für die entsprechenden Radwege keine mikroskopische Analyse vorgenommen, um Verzerrungen zwischen beiden Zeiträumen zu vermeiden. In den verbleibenden Städten erfolgte die mikroskopische Unfallanalyse mittels Unfallhergangsbeschreibungen oder -skizzen. In Berlin erfolgte diese über die lagegetreu verorteten Unfälle sowie die von der Berliner Polizei vergebene Unfallsymbolik, welche ebenso Rückschlüsse auf den Unfallhergang und die Flächennutzung der Radfahrer zulassen. 
Unter Berücksichtigung der Datenverfügbarkeit ergaben sich die nachfolgenden vier wesentlichen Merkmale als Ergebnis der mikroskopischen Unfallanalyse:

- Hergang vorher oder nachher nicht verfügbar

- Hergang verfügbar, Flächennutzung unbekannt

- Flächennutzung Fahrbahn

- Flächennutzung Seitenraum

Entsprechend dieser - aus der mikroskopischen Unfallanalyse generierten Informationen - erfolgte anschließend die makroskopische Unfallanalyse nach den wesentlichen Unfallmerkmalen der Radunfälle mit Personenschaden. Eine schematische Darstellung der Ergebnisse dieser detaillierten Unfallanalyse (gemeinsame makroskopische und mikroskopische Analyse) enthält Abbildung 24. Dabei werden die Ergebnisse der makroskopischen Unfallmerkmale (vgl. Kapitel 4.1.7) differenziert nach dem Zeitraum vor bzw. nach Aufhebung der Radwegbenutzungspflicht auf der XAchse dargestellt. Die Y-Achse beinhaltet im Diagramm links (Abbildung 24) neben der Gesamtsumme der absoluten Radunfallanzahl pro Jahr des makroskopischen Unfallmerkmales (gesamte Säule) die Differenzierung in die einzelnen Flächennutzungen aus der mikroskopischen Unfallanalyse (Stapel). Zudem erfolgten relative Betrachtungen der einzelnen Säulen (Diagramm rechts, Abbildung 24) mit einer Normierung auf $100 \%$, wobei nur bekannte Flächennutzungen berücksichtigt wurden. Somit konnten sowohl die gesamten Veränderungen der makroskopischen Unfallmerkmale (Angabe in Prozent unter dem Merkmal in Klammern, wenn Summe der Unfallanzahl dieses Merkmals pro Jahr kleiner als 20) als auch einzelne Verschiebungen zwischen den Flächennutzungen vor und nach Aufhebung der Radwegebenutzungspflicht bewertet werden.
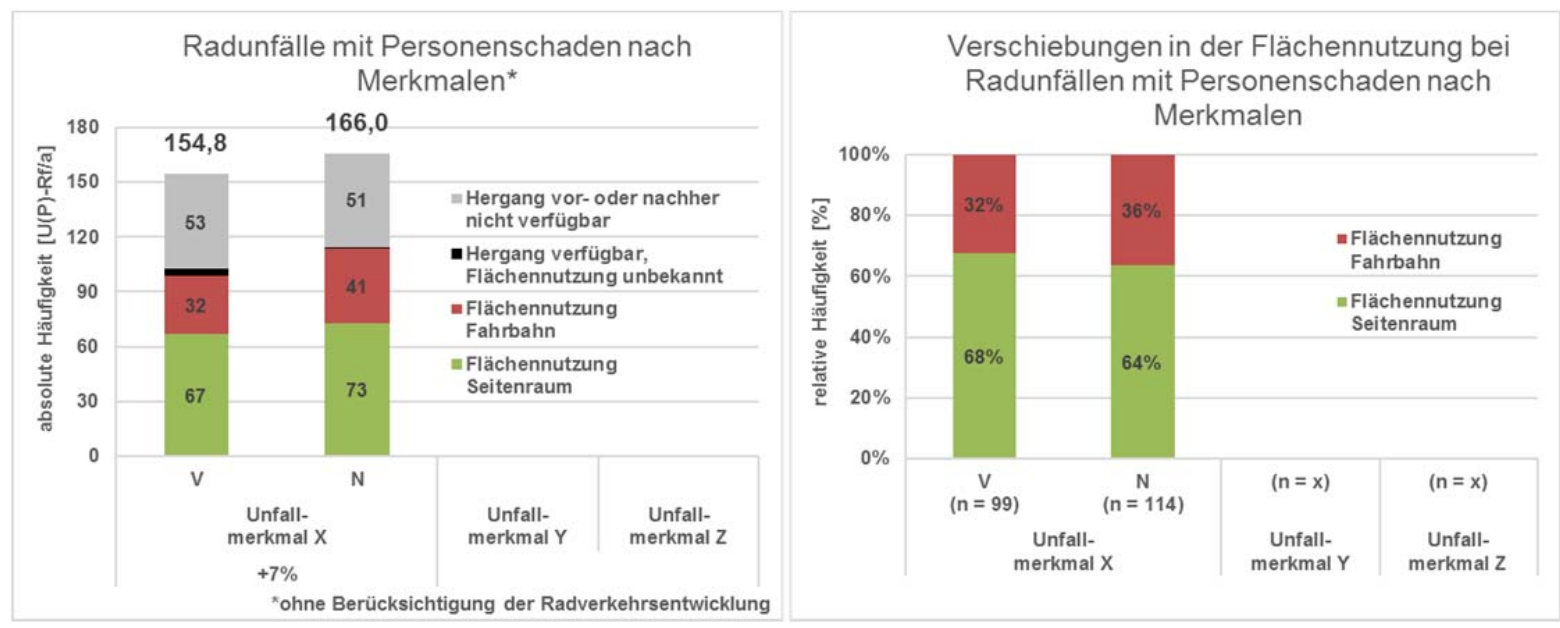

Abbildung 24: Auswerteschema der makroskopischen und mikroskopischen Unfallanalyse

Neben der Identifizierung der Flächennutzung der am Unfall beteiligten Radfahrer konnte erst mit Hilfe der mikroskopischen Unfallanalyse die tatsächliche Zugehörigkeit der Radunfälle zu den Untersuchungsstrecken abschließend geklärt werden. Durch die Knotenpunktdefinition nach Kapitel 4.1.2 konnten in den 890 Radunfällen noch Unfälle der - die Untersuchungsstrecke - kreuzenden Straßen enthalten sein, welche nach der mikroskopischen Unfallanalyse entfernt wurden. Der verbleibende Fehler in den drei Städten ohne mikroskopische Unfalldaten ist aufgrund weniger Unfälle gering. Somit reduzierte sich das final untersuchte Unfallkollektiv auf den betrachteten 108 Radwegen auf 741 Radunfälle mit Personenschaden. Insgesamt entfielen von diesen Radunfällen 397 Unfälle auf den Zeitraum vor Aufhebung der Radwegebenutzungspflicht (Durchschnitt 2,80 Jahre, vgl. Abbildung 23) sowie 344 Unfälle auf den Zeitraum nach Aufhebung der Radwegebenutzungspflicht (Durchschnitt 2,51 Jahre gemäß Abbildung 23). 


\subsection{Vorher-Nachher-Untersuchung des Verkehrsverhaltens}

Neben den Unfalluntersuchungen vor und nach Aufhebung der Radwegebenutzungspflicht wurden Verkehrsverhaltensanalysen an weiteren 10 Radwegen angestrebt, an denen erst im Rahmen der Projektlaufzeit verkehrsrechtliche Änderungen der Benutzungspflicht vorgesehen waren. Dies ermöglichte in Zusammenarbeit mit den Städten eine Begleituntersuchung dieser Maßnahmen. Die Streckenvorschläge erfolgten dabei ebenfalls seitens der kommunalen Entscheidungsträger im Nachgang zur 2. Telefonbefragung (vgl. Kapitel 3.4). Mit dieser zusätzlichen Analyse sollten neben dem Unfallgeschehen detailliertere Erkenntnisse zu Verhaltensänderungen durch einen Vergleich des Verkehrsverhaltens - hier vorrangig Verkehrskonflikte und Flächennutzung der Radfahrer - an Radwegen mit und ohne Benutzungspflicht generiert werden. Es galt hier zu bewerten, ob die Aufhebung der Benutzungspflicht zu neuen Konflikten führt oder zur Minderung des Konfliktpotenzials beiträgt. Damit sollten Hinweise auf mehr oder weniger kritische Interaktionen gewonnen werden, die allein aus den Unfallanalysen nicht ableitbar waren. Des Weiteren ermöglichten die Verhaltensbeobachtungen Einblicke in die tatsächliche Flächennutzung der Radfahrer im Vergleich von Radwegen mit und ohne Benutzungspflicht.

\subsubsection{Auswahl von benutzungspflichtigen Radwegen}

Von denen im Rahmen der Unfallanalyse betrachteten 8 Städten machten 5 Städte (Berlin, Düsseldorf, Hamburg, Nürnberg, Paderborn) nähere Angaben zu Radwegen an denen die Benutzungspflicht während der Projektlaufzeit aufgehoben werden sollte. Für alle genannten Radwege erfolgte entsprechend Kapitel 4.1.1 eine Prüfung der Eignung. Dabei wurden erneut Zweirichtungsradwege und gemeinsame Geh- und Radwege ausgeschlossen. Weiterhin blieben zusätzlich Radwege unberücksichtigt, an denen bereits vorbereitende Baumaßnahmen zur Aufhebung der Radwegebenutzungspflicht begonnen wurden oder bei denen der Zeitpunkt der tatsächlichen Aufhebung noch unbekannt und die Begleituntersuchung im Rahmen des Projektes somit ungewiss war. Für die nun noch verbleibenden 32 Radwege galten die folgenden Auswahlkriterien:

\section{Kriterium 1:}

Wesentliches Auswahlkriterium war die Begleitmaßnahme mit Aufhebung der Radwegebenutzungspflicht. Vorrangig sollten einzelne Begleitmaßnahmen untersucht werden, um auch Einzeleffekte bewerten zu können. Ausnahme bildete hier die Überlagerung aus der Markierung von Radfahrstreifen und dem Rückbau der Radwege, welche nicht einzeln auftraten.

\section{Kriterium 2:}

Jede Begleitmaßnahme sollte vorzugsweise mindestens einmal untersucht werden.

\section{Kriterium 3:}

Bei nun noch mehreren potentiell geeigneten Untersuchungsstrecken innerhalb einzelner Cluster wurden die Strecken mit dem größten Untersuchungspotential ausgewählt (u.a. ausreichend hohes Radverkehrsaufkommen).

Ähnlich der Streckenauswahl für die Unfallanalysen blieben auch hier einzelne Cluster aufgrund ausgebliebener Streckenvorschläge der Städte unbesetzt. Somit konnten im Rahmen der Verkehrsverhaltensanalysen Radwege mit Anordnungen der Beschilderung Gehweg Radfahrer frei, der Anordnung nichtamtlicher Hinweisschilder sowie einer Beschränkung der zulässigen Höchstgeschwindigkeit nicht weiter betrachtet werden. 


\subsubsection{Alternative Vorgehensweisen}

Im Verlauf des vorliegenden Projektes haben sich an den ursprünglich ausgewählten 10 benutzungspflichtigen Radwegen teilweise Verzögerungen bei der Umsetzung der Aufhebung der Radwegebenutzungspflicht ergeben, weswegen die angestrebten Vorher-Nachher-Vergleiche nicht ausnahmslos realisiert werden konnten und alternative Analysemethoden zur Anwendung kamen.

Da zum Zeitpunkt des Bekanntwerdens dieser Verzögerungen bereits alle ursprünglich geplanten Vorher-Erhebungen vorlagen, wurden Vergleichsstrecken zu den Radwegen gesucht, deren Benutzungspflicht zeitlich nicht mehr bis zum Ende des vorliegenden Projektes aufgehoben wurde. Neben den direkten Vergleichen vor und nach Aufhebung der Radwegebenutzungspflicht erfolgten dementsprechend auch Vergleiche von ähnlich charakterisierten Radwegen mit und ohne Benutzungspflicht. Dabei handelte es sich zumeist um direkte Vorlauf- oder Folgestrecken ohne Radwegebenutzungspflicht der ursprünglich ausgewählten noch benutzungspflichtigen Radwege. Damit war sichergestellt, dass die Straßencharakteristik und das Verkehrsgeschehen der Vergleichsstrecken miteinander vergleichbar sind. In einem Fall wurde der benutzungspflichtige Radweg mit dem nicht benutzungspflichtigen Radweg der anderen Fahrtrichtung derselben Straßen verglichen.

Für einen weiteren Teil der Untersuchungsstrecken ohne erfolgter Aufhebung der Benutzungspflicht wurde abschließend zur Spezifizierung der Besonderheiten an (Ersatz-)Radwegen, bei denen sich im Vorher-Nachher-Vergleich der Unfallanalyse deutliche Veränderungen eingestellt haben, nur Erhebungen nach Aufhebung der Radwegebenutzungspflicht durchgeführt, ohne hierfür einen direkten Vergleichsfall zu suchen. Hieraus sollten weitere - die Unfallanalyse erklärende Erkenntnisse generiert werden. Im Ergebnis wurden mit den zuvor beschriebenen unterschiedlichen Herangehensweisen die folgenden Auswertungen an den Radwegen ermöglicht:

- 2 Radwege mit direktem Vorher-Nachher-Vergleich bezüglich der Radwegebenutzungspflicht, davon

o 1 Radweg mit Markierung eines Radfahrstreifens (inkl. Rückbau des Radweges) als Begleitmaßnahme

o 1 Radweg mit Markierung von Radfahrerpiktogrammen auf dem nicht benutzungspflichtigen Radweg und am Fahrbahnrand als Begleitmaßnahme

- 4 Radwege mit einer Vorher-Untersuchung des Verkehrsverhaltens an den ursprünglich ausgewählten benutzungspflichtigen Radwegen mit alternativem Mit-Ohne-Vergleich bezüglich der Radwegebenutzungspflicht an Vorlauf- oder Folgestrecken, davon

o alle 4 nicht benutzungspflichtige Radwege ohne weitere Begleitmaßnahmen

- 4 Radwege mit alternativer Nachher-Untersuchung (ohne Vergleich) an 4 nicht benutzungspflichtigen Radwegen in Anlehnung an die Unfallanalysen, davon

o 3 nicht benutzungspflichtige Radwege ohne weitere Begleitmaßnahmen

o 1 Radweg mit Markierung von Radfahrerpiktogrammen auf dem nicht benutzungspflichtigen Radweg als Begleitmaßnahme

Das Erhebungskonzept ist unabhängig der eben beschriebenen Herangehensweisen identisch.

\subsubsection{Erhebungskonzept}

Nach Auswahl der Untersuchungsstrecken erfolgten im Jahr 2016 bei vorhandener Benutzungspflicht der betreffenden Radwege die Videoerhebungen des Vorher- (bzw. Mit-) Szenarios. Bei allen Erhebungen wurde das Erhebungsdesign nach Abbildung 25 angewendet. 


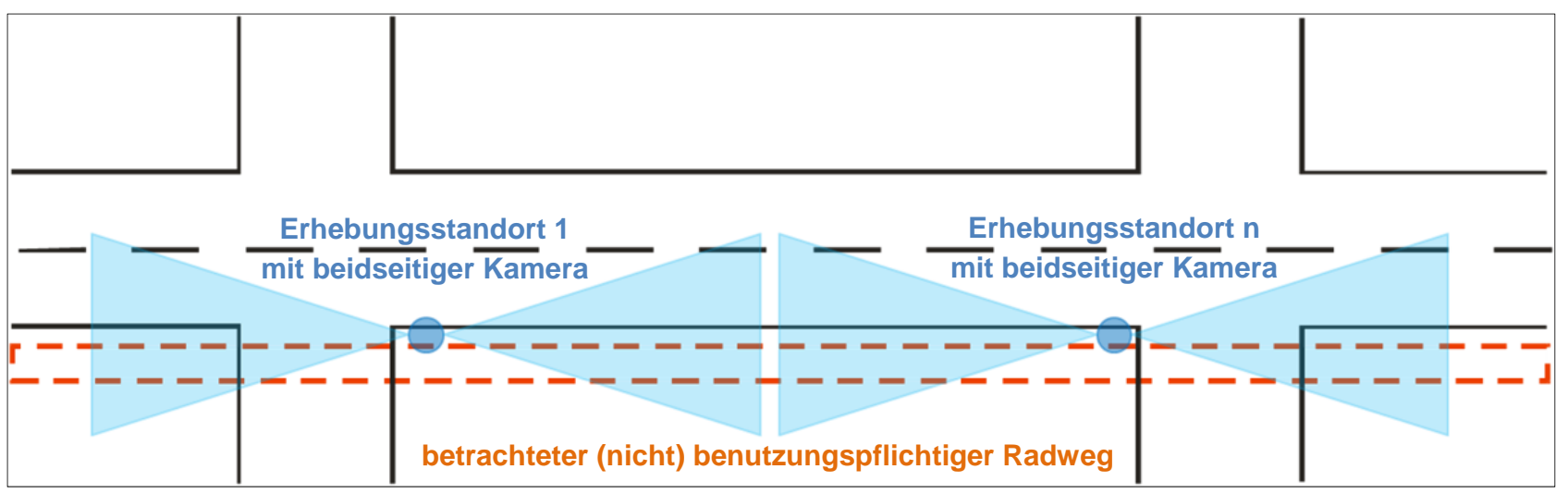

Abbildung 25: Schema des Erhebungsdesigns für die Verkehrsverhaltensanalysen

Ziel der Erhebungen war die - in Abhängigkeit der Streckeneigenschaften weitestgehend lückenlose - Abdeckung des zu untersuchenden Radweges und der angrenzenden Fahrstreifen mit Kamerasichtfeldern. Hier stand im Vordergrund, die den Streckenabschnitt befahrenden Radfahrer über die gesamte Streckenlänge zu beobachten und deren Flächennutzung sowie mögliche auftretende Konflikte mit anderen Verkehrsteilnehmern zu ermitteln. Da mit Verfolgungsfahrten per Rad nur eine kleine Stichprobe aller Radfahrer und somit auch insgesamt nur wenige Konflikte beobachtet werden können, wurde dieses Konzept nach einer Piloterhebung nicht weiterverfolgt und ausschließlich stationäre Erhebungen entsprechend Abbildung 25 durchgeführt.

Der Fokus der späteren Auswertungen lag auf dem tatsächlichen Konfliktgeschehen von Radfahrern, was in Kapitel 4.3.4 näher beschrieben wird. Die Verkehrsverhaltensbeobachtungen erfolgten in den verkehrsreichen Zeiträumen von 7-10 Uhr sowie 15-18 Uhr, in denen auch die meisten Radfahrer und Interaktionen zu erwarten waren. Entsprechend der Unfallanalysen wurden auch bei den Verkehrsverhaltensanalysen die Bereiche der knotenpunktfreien Strecke und der Knotenpunkte differenziert erhoben und bewertet. Somit konnten die spezifischen Besonderheiten dieser Bereiche im Unfallgeschehen auch mit den Verkehrsverhaltensanalysen verglichen werden.

Für die Videoerhebungen des Nachher-Szenarios war vorgesehen, nach den positiven Rückmeldungen der betreffenden Städte über den Vollzug der Aufhebung der Radwegebenutzungspflicht, den gleichen Erhebungsaufbau je Untersuchungsstrecke zu reproduzieren, wie er auch bei den Erhebungen des Vorher-Szenarios angewendet wurde. Dabei wurde nach dem Vollzug der Aufhebung der Radwegebenutzungspflicht eine Eingewöhnungsphase der Verkehrsteilnehmer von ca. 3 Monaten berücksichtigt. Für die Mit-Ohne-Vergleiche bzw. die Analysen an Radwegen ohne Benutzungspflicht basierend auf den Unfallanalysen wurden Erhebungen entsprechend Abbildung 25 durchgeführt und den Streckenverhältnissen der Vergleichs- bzw. Ersatzstrecken angepasst.

Parallel zu den Videoerhebungen an den betrachteten Radwegen erfolgte eine Vor-OrtBefragung von Radfahrenden sowie von Fußgängern und an der Untersuchungsstrecke parkenden Kraftfahrzeugführern. Damit konnten die Einschätzungen des gesamten Spektrums der Verkehrsteilnehmer, welche von der Aufhebung der Radwegebenutzungspflicht potentiell betroffen sein können, ermittelt werden. Der Schwerpunkt lag hierbei jedoch bei den Radfahrern. Im Zentrum des Interesses bei den Befragungen standen vorrangig die Motive des eigenen Verkehrsverhaltens (z.B. bei Radfahrern die Gründe für die Nichtnutzung der Fahrbahn nach Aufhebung der Radwegbenutzungspflicht) und die generelle Wahrnehmung der verkehrsrechtlichen Rahmenbedingungen. Zudem wurde eine Einschätzung des Verkehrsverhaltens der jeweils anderen Verkehrsteilnehmer und das wahrgenommene Konfliktpotential an knotenpunktfreier Strecke sowie den Knotenpunkten des untersuchten Abschnittes abgefragt. 


\subsubsection{Verkehrsverhaltensanalyse}

Die Auswertungen der nach Kapitel 4.3.3 erhobenen Daten erfolgten durch eine Verkehrszählung und eine Konfliktanalyse, welche nachfolgend detaillierter beschrieben werden.

Die Verkehrszählungen dienten grundlegend der Identifizierung von generellen Bezugsgrößen für die anschließende Konfliktanalyse. Dafür wurden die Verkehrsstärken des Kraftfahrzeug- und Radverkehrs während der in Kapitel 4.3.3 genannten Erhebungszeiten ermittelt. Die Zählungen erfolgten dabei an einem für die betreffende Strecke maßgebendem Querschnitt. Für den Kraftfahrzeugverkehr wurden die Verkehrszahlen differenziert nach den Fahrzeugklassen:

- Mofa/Krad (Zweiräder),

- Pkw/Lieferwagen (Fahrzeuge unter 3,5 t) sowie

- $\quad$ Lkw/Busse (Fahrzeuge über 3,5 t).

Bei den Radverkehrszählungen wurden darüber hinaus noch die folgenden Daten ermittelt, welche für die Beantwortung spezieller Fragestellungen in Bezug auf die Radwegebenutzungspflicht von Bedeutung waren:

- Flächennutzung der Radfahrer

- augenscheinliches Alter (<10,10-18,19-65, $>65$ Jahre) und Geschlecht der Radfahrer

- Regelverstöße der Radfahrer (z.B. regelwidriges Linksfahren)

Darüber hinaus konnte in Verbindung mit dem Status der Radwegebenutzungspflicht und den Erhebungen der Flächennutzung indirekt auf weitere Regelverstöße geschlussfolgert werden, wenn beispielsweise bei vorhandener Radwegbenutzungspflicht auf der Fahrbahn oder unabhängig der Radwegebenutzungspflicht auf dem Gehweg gefahren wurde.

Die anschließende Konfliktanalyse basiert auf folgenden Grundlagen und Festlegungen. Ein Konflikt ist dabei in Anlehnung an Erke et al. [1985] ein kritisches Verkehrsmanöver, um eine Kollision zu vermeiden. Zudem wurde bei den Analysen in leichte und schwere Konflikte sowie Unfälle unterschieden. Leichte Konflikte sind dabei in Bezug auf Kraftfahrzeugführer oder Radfahrer gekennzeichnet durch kontrolliertes Bremsen, Beschleunigen oder Ausweichen, bei Fußgängern durch Stehenbleiben, Zurückgehen, Beschleunigen oder Abbremsen. Schwere Konflikte ergeben sich für Kraftfahrzeugführer durch starkes Bremsen oder Beschleunigen und/oder abruptes Ausweichen. Bei Radfahrern kommen neben den eben genannten Verhaltensweisen noch Verreißen des Lenkers oder Abspringen vom Fahrrad hinzu. Schwere Konflikte bei Fußgängern zeigen sich durch plötzliches Stehenbleiben oder Beschleunigen sowie abruptes Ausweichen oder Zurückspringen. Unfälle wären entsprechend die schwerwiegendsten beobachtbaren Verkehrsmanöver, bei denen eine Kollisionsvermeidung nicht mehr erfolgreich war (vgl. Erke et al. 1985). Die Systematik der Auswertungen enthält Abbildung 26.

\begin{tabular}{|c|c|}
\hline \multicolumn{3}{|c|}{ Bezugsgröße Radverkehrsaufkommen (Radfahrten) } \\
\hline Regelkonforme Radfahrt & Nicht regelkonforme Radfahrt \\
\hline \multicolumn{3}{|c|}{ Verkehrskonflikte (kritische Fahrmanöver) } \\
\hline Leichter Konflikt & \multicolumn{3}{|c|}{ Schwerer Konflikt } \\
\cline { 2 - 3 } & \multicolumn{3}{|c|}{ Ohne Unfall } & Unfall \\
\hline
\end{tabular}

Abbildung 26: Systematik der Auswertungen zur Verkehrsverhaltensanalyse 
Zur Beurteilung der Konfliktsituation wurde als Bezugsgröße das ermittelte Radverkehrsaufkommen der Verkehrszählungen herangezogen. Neben den bereits genannten allgemeinen Definitionen wurden weiterhin im Falle eines beobachteten Konfliktes die folgenden Informationen festgehalten:

- Codierung der Untersuchungsstrecke

- Status der Radwegebenutzungspflicht (vor/nach Aufhebung bzw. mit/ohne Radwegebenutzungspflicht)

- Charakteristik der Örtlichkeit (Knotenpunkte, knotenpunktfreie Strecke, Haltestellen)

- Uhrzeit

- Konfliktschwere

- Flächennutzung des Radfahrers

- Regelverstöße des Radfahrers

- Konfliktpartner, Konfliktursache, Konfliktbeschreibung, Konfliktlösung

- Einordnung des Konflikts gemäß 3-stelligem Unfalltyp entsprechend FGSV [2003]

- Alter (<10,10-18,19-65, >65 Jahre) und Geschlecht des Radfahrers

Als Zusatzinformationen dienen somit neben den allgemeinen Merkmalen der Örtlichkeit (u.a. Differenzierung in Knotenpunkte und knotenpunktfreie Strecke entsprechend der Schwerpunkte der Unfallanalyse), des Konfliktes (u.a. Beschreibung und Lösung des Konfliktes bzw. der Situation) sowie des konfliktbeteiligten Radfahrers auch der entsprechend der Konfliktsituation zuordenbare Unfalltyp, welcher im Falle einer nicht erfolgreichen Kollisionsvermeidung zugetroffen hätte. Damit konnten die Konfliktsituationen vereinfacht beschrieben und abschließend auch Vergleiche zu den durchgeführten Unfallanalysen hergestellt werden. 


\section{Ergebnisse der Unfallanalyse}

In diesem Kapitel werden nach der Vorstellung der allgemeinen Tendenzen im Unfallgeschehen und den zugehörigen Sensitivitätsanalysen die Ergebnisse der Unfallkenngrößenberechnung auf den untersuchten Radwegen vorgestellt. Anschließend werden die Veränderungen im Radunfallgeschehen sowie die Verschiebungen in der Flächennutzung durch Aufhebung der Radwegebenutzungspflicht mittels gemeinsamer makroskopischer und mikroskopischer Unfallanalyse beschrieben und bewertet. Dabei erfolgt ergänzend zu der allgemeinen Unfallentwicklung und der Unfallkenngrößenberechnung eine Differenzierung nach weiteren Unfallmerkmalen sowie nach der tatsächlichen Flächennutzung der am Unfall beteiligten Radfahrer.

\subsection{Allgemeine Tendenzen im Unfallgeschehen}

Im Gesamtunfallgeschehen an den untersuchten Radwegen ergab der Vergleich der Zeiträume vor und nach Aufhebung der Radwegebenutzungspflicht eine geringfügige Abnahme um -6\% (Abbildung 27). Auch das Unfallkollektiv ohne Radbeteiligung war mit -12 \% rückläufig. Radunfälle haben dagegen nach Aufhebung der Radwegebenutzungspflicht um $7 \%$ zugenommen. Entgegen des Trends im Gesamtunfallgeschehen hat sich eine Erhöhung der Radunfälle eingestellt.

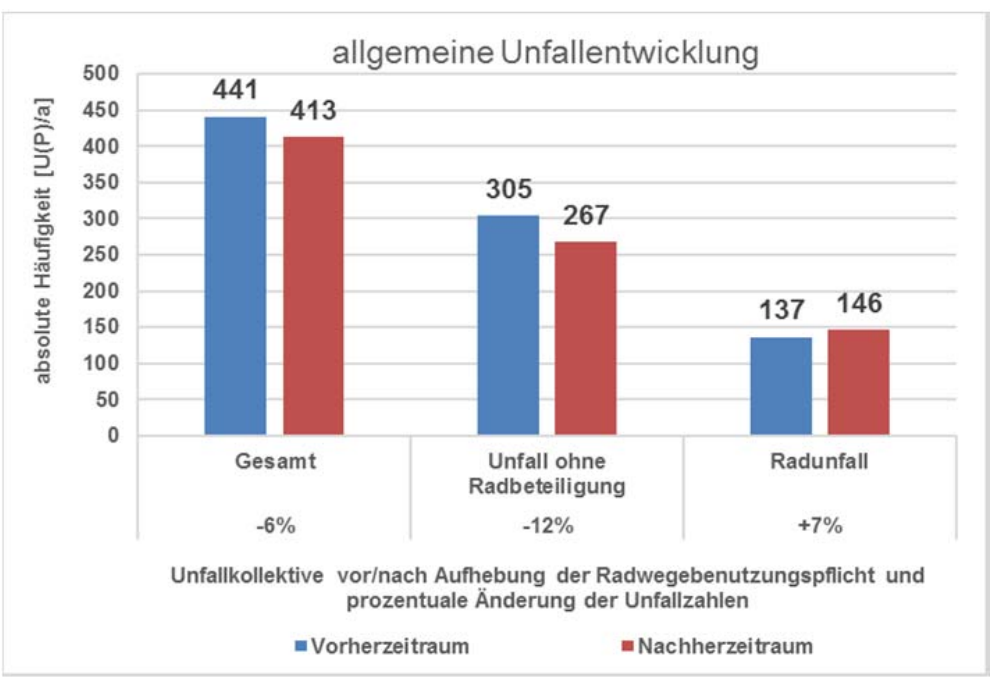

Abbildung 27: Unfallentwicklung mit Aufhebung der Radwegebenutzungspflicht

Nach Abbildung 28 sind Unfälle an Knotenpunkten in allen Teilunfallkollektiven mit etwa $75 \%$ maßgebend. Innerhalb der Teilunfallkollektive ergab sich auf der knotenpunktfreien Strecke im Gesamtunfallgeschehen eine leichte Zunahme, an Knotenpunkten zeigte sich dagegen eine Abnahme. Bei Unfällen ohne Radverkehrsbeteiligung wurde generell eine Abnahme festgestellt. Insgesamt sind jedoch die Veränderungen im Unfallgeschehen auf der knotenpunktfreien Strecke im Vergleich zu den Knotenpunkten gering.

Bei den Radunfällen zeigte sich dagegen eine Zunahme auf der knotenpunktfreien Strecke und an den Knotenpunkten. Die Zunahme ist dabei auf der knotenpunktfreien Strecke am stärksten. Ob es sich hierbei um eine Auswirkung der Aufhebung der Radwegebenutzungspflicht handelt oder die Zunahme der Unfälle mit der allgemeinen Unfallentwicklung bzw. der Zunahme des Radverkehrs in den Untersuchungsstädten erklärt werden kann, wird in den Kapiteln 5.2 und 5.3 erörtert. 


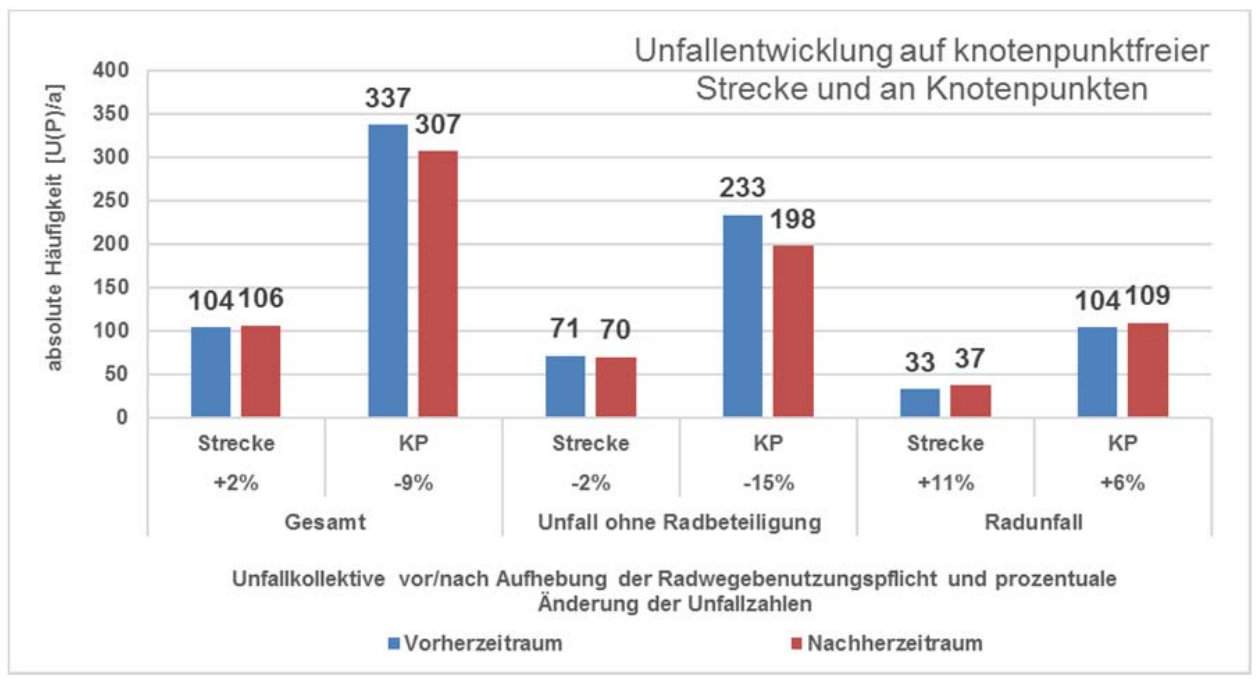

Abbildung 28: Unfallentwicklung differenziert nach knotenpunktfreier Strecke und Knotenpunkten

\subsection{Unfallentwicklung im Untersuchungskollektiv und Sensitivitätsanalyse}

Die Ergebnisse der Veränderungen der Radunfälle pro Jahr mit Aufhebung der Radwegebenutzungspflicht an den einzelnen untersuchten Radwegen enthält Abbildung 29.

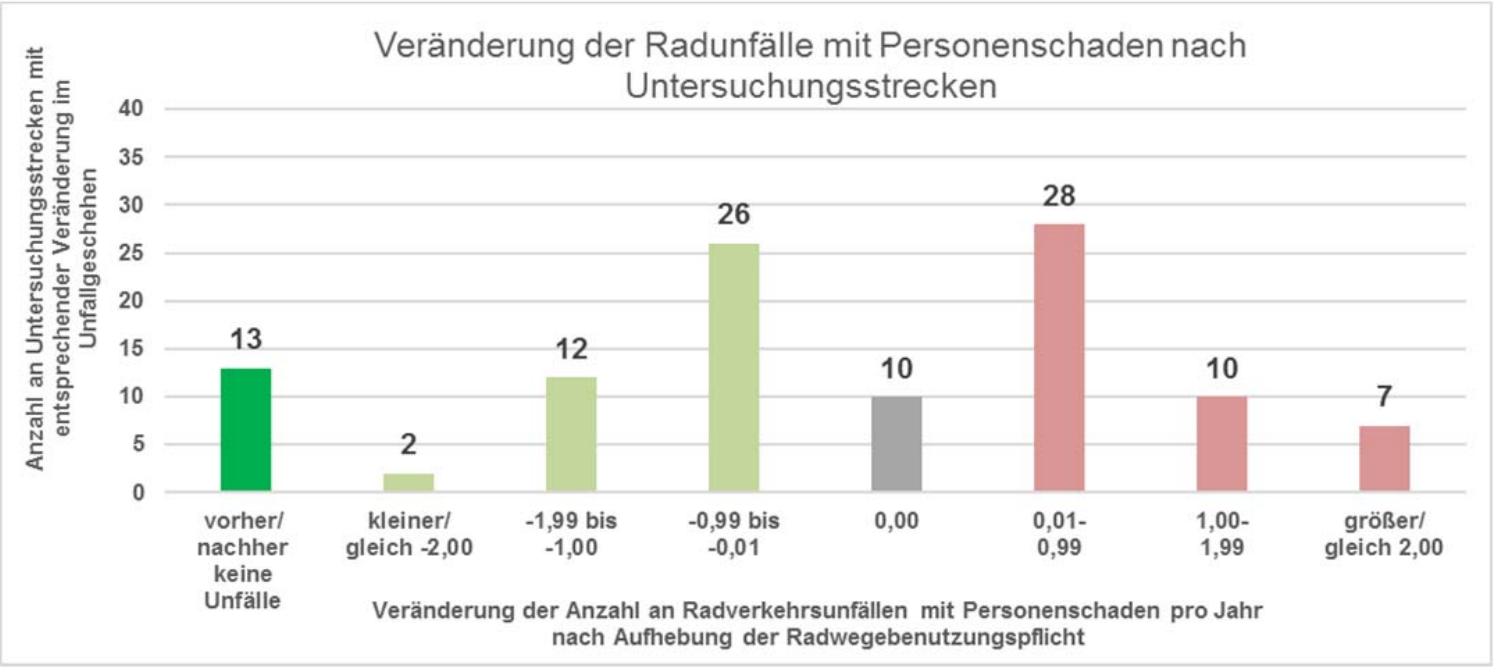

Abbildung 29: Veränderungen im Radunfallgeschehen auf einzelnen Untersuchungsstrecken

Von den 108 Radwegen wiesen 13 Radwege vor und nach Aufhebung der Radwegebenutzungspflicht keine Radunfälle auf. Die Verteilung der verbleibenden Radwege ließ eine Normalverteilung von Unfallzunahme bzw. -abnahme nach Aufhebung der Radwegebenutzungspflicht erkennen. Das Untersuchungskollektiv enthält entsprechend keinen Auswahlfehler bezüglich bestimmter Unfallbelastungen. Jedoch zeigt sich eine leichte Schiefe hin zu Radwegen mit stark zunehmenden Radunfällen. An 7 Radwegen war eine Zunahme von mehr als 2 Radunfällen pro Jahr zu verzeichnen. Eine Abnahme um die gleiche Größenordnung trat nur an 2 Radwegen auf.

Der Vergleich der Entwicklungen von Rad- und Gesamtunfallgeschehen der Unfallkategorien 1 bis 3 zeigte für die einzelnen untersuchten Radwege über die einzelnen Jahre hinweg sehr heterogene Ergebnisse. Der zur Bewertung herangezogene Quotient aus Rad- und Gesamtunfallgeschehen war hier stark schwankend, insbesondere bei geringen Unfallzahlen. Eine Aggregation der Radwege nach den Jahren der Aufhebung der Radwegebenutzungspflicht und den gesamten Vorher-Nachher-Zeiträumen sowie die Vorgabe einer Mindestanzahl von Radunfällen ergab ebenfalls 
noch sehr heterogene Kennzahlen. Einheitliche Trends für Einzelstrecken und Einzeljahre konnten nicht abgeleitet werden (vgl. auch Abbildung 29). Vergleiche des Quotienten aus Rad- und Gesamtunfallgeschehen auf Grundlage von Einzelstrecken und Einzeljahren mit den Trends im Unfallgeschehen der Städte waren daher nicht zielführend.

Die Aggregation über das gesamte Streckenkollektiv ergab eine mittlere Steigerung des Quotienten aus Rad- und Gesamtunfallgeschehen von 0,28 vor auf 0,37 nach Aufhebung der Radwegebenutzungspflicht. Demzufolge war der Anteil der Radunfälle am Gesamtunfallgeschehen auf den betrachteten Untersuchungsstrecken tendenziell steigend. Gemäß Abbildung 27 ist für diese Entwicklung die Abnahme von Unfällen ohne Radverkehrsbeteiligung und die Zunahme von Radunfällen gleichermaßen ursächlich. Zur weitergehenden Bewertung dieser Tendenzen im Unfallgeschehen der ausgewählten Radwege erfolgte eine Gegenüberstellung mit den Quotienten aus Radunfall- und Gesamtunfallgeschehen für die Gesamtstadt sowie für alle innerörtlichen Unfälle mit Personenschaden in Deutschland. Das Ergebnis zeigt Abbildung 30.

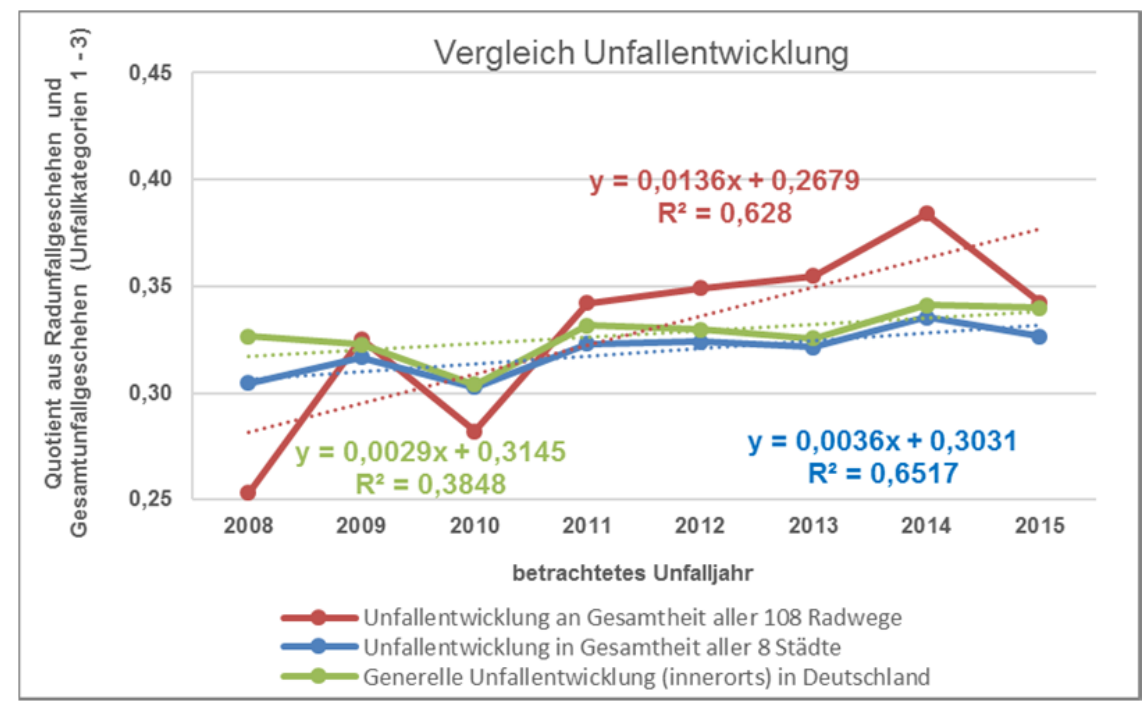

\section{Abbildung 30: Entwicklung des Quotienten aus Rad- und Gesamtunfallgeschehen auf den betrachteten Untersuchungstrecken, in den ausgewählten Städten sowie in Gesamtdeutschland}

Insgesamt ergab sich in den 3 Unfallkollektiven eine Zunahme des Quotienten aus Rad- und Gesamtunfallgeschehen von 2008 bis 2015. Über die betrachteten Jahre hinweg ist der Anteil von Radunfällen an allen Unfällen mit Personenschaden angestiegen. Die Bestimmtheitsmaße liegen über alle Kollektive hinweg bei mindestens 0,38. Für die Gesamtunfallentwicklung in Deutschland zeigte sich der geringste Anstieg der Regressionsgeraden und somit die geringste Zunahme des zur Bewertung herangezogenen Quotienten. Ein etwas stärkerer Anstieg war im Unfallgeschehen der 8 ausgewählten Städten zu verzeichnen. Als Grund wurde die Betrachtung von Städten mit höherem Radverkehrsaufkommen in der Untersuchung angenommen. Nennenswerte Unterschiede der Unfallentwicklungen zwischen einzelnen Städten ergaben sich nicht. An den ausgewählten 108 Radwegen zeigte sich ein ca. 4-mal stärkerer Anstieg des Quotienten aus Rad- und Gesamtunfallgeschehen als in den Städten bzw. in Deutschland. Im ausgewählten Kollektiv war daher von einer stärkeren Zunahme der Radunfälle im Vergleich zum Trend aller Unfälle auszugehen.

Durch die steigenden Tendenzen verdeutlichen sich mehr Radunfälle in jüngeren Jahren. Hier wird vermutet, dass die Zunahme der Radunfälle mit Personenschaden mit einem gestiegenen Radverkehrsaufkommen der vergangenen Jahre erklärbar ist, ein Zusammenhang, der bereits von GDV [2015] nachgewiesen wurde. Daher wird die Radverkehrsentwicklung bei der folgenden Unfallkenngrößenberechnung berücksichtigt und deren Einfluss geprüft. 


\subsection{Veränderungen im Radunfallgeschehen}

Dieses Kapitel bildet den Hauptbestandteil der Unfallanalyse und beinhaltet die Ergebnisse der Unfallkenngrößenberechnung nach ausgewählten Streckenmerkmalen sowie nach einem Exkurs zur tatsächlichen Fahrbahnnutzung der Radfahrer an nicht benutzungspflichtigen Radwegen die Auswertungen der detaillierten makroskopischen und mikroskopischen Unfallanalyse.

\subsubsection{Ergebnisse der Unfallkenngrößenberechnung}

Als Bezugsgröße des Verkehrsaufkommens wurden die Radverkehrsstärken verwendet. Bewertet wird somit nachfolgend das Risiko für Radfahrer, in einen Unfall verwickelt zu werden. In Kapitel 5.3.1.1 werden zunächst die Unfallkenngrößen ohne Projektion der Radverkehrsstärken dargestellt. Anschließend erfolgte eine Projektion der Radverkehrsstärken zwischen dem Vorher- und Nachher-Zeitraum (vgl. Kapitel 4.1.6 sowie Kapitel 5.2), wodurch die Radverkehrsentwicklung einbezogen wurde. Die Ergebnisse dieses zweiten Berechnungsansatzes folgen in Kapitel 5.3.1.2.

\subsubsection{Unfallkenngrößen ohne Projektion der Radverkehrsstärken}

Die Ergebnisse ohne Projektion der Radverkehrsstärken differenziert nach Art der Begleitmaßnahme enthält Anhang $C$. Analog zur Unfallentwicklung nach Abbildung 28 weisen die knotenpunktfreien Strecken und die Knotenpunkte über alle 108 betrachteten Radwege hinweg im Zeitraum nach Aufhebung der Radwegebenutzungspflicht höhere Unfallraten auf als zuvor.

Eine ansteigende Tendenz des fahrleistungsbezogenen Unfallrisikos zeigte sich auch bei den einzelnen Begleitmaßnahmen, mit Ausnahme der markierten Schutzstreifen und Radfahrerpiktogramme an Knotenpunkten. Bei Schutzstreifen an Knotenpunkten waren die Unfallraten nach Aufhebung der Radwegebenutzungspflicht geringer. Dabei erfolgte zu gleichen Teilen ein bzw. kein Rückbau des Radweges. Die Abnahme der Unfallrate war ausschließlich auf Schutzstreifen mit zurückgebauten Radweg zurückzuführen. Bei dualer Führung beider Radverkehrsanlagen blieb die Unfallrate konstant. Bei der Markierung von Radfahrerpiktogrammen ergab sich an Knotenpunkten keine Änderung der Unfallrate. Die Radfahrerpiktogramme wurden hier an 2 von 15 Radwegen am Fahrbahnrand markiert. Die dargestellten Tendenzen werden somit maßgeblich von Radfahrerpiktogrammen auf dem nicht benutzungspflichtigen Radweg bestimmt. Belastbare Unterschiede zwischen Radfahrerpiktogrammen auf dem Radweg und am Fahrbahnrand zeigten sich nicht.

Bei den Unfallkostenraten ergab sich auf der knotenpunktfreien Strecke eine Verringerung, an den Knotenpunkten dagegen eine Erhöhung der Kenngrößen im Gesamtkollektiv. Entsprechende Entwicklungen wurden auch bei der Unterscheidung nach Begleitmaßnahmen festgestellt, außer bei Schutzstreifen und Radfahrerpiktogrammen.

Beim Vergleich von Unfalldichten und -kostendichten mit den Unfallraten und -kostenraten zeigten sich keine Unterschiede. Demzufolge sind die Tendenzen der fahrleistungsbezogenen und längenbezogenen Unfallkenngrößen identisch. Auf die Unfalldichten und -kostendichten wird nachfolgend nur bei wesentlichen Unterschieden zu den Unfallraten und -kostenraten eingegangen.

\subsubsection{Unfallkenngrößen mit Projektion der Radverkehrsstärken}

\section{Abschätzung der Radverkehrsentwicklung}

Nachfolgend wurde die Radverkehrsentwicklung in die Unfallkenngrößenberechnung einbezogen. Hierzu erfolgte eine Projektion der Radverkehrsstärken zwischen dem Zeitraum vor bzw. nach Aufhebung der Radwegebenutzungspflicht. Dabei wurden die jährlichen Radverkehrsentwicklungen basierend auf den Änderungen der Verkehrsleistungen im Radverkehr nach SrV zugrunde ge- 
legt (Tabelle 6). Da Nürnberg und Paderborn nicht an den SrV-Befragungen teilnehmen, wurde hier die Gesamtentwicklung der Verkehrsleistung des Radverkehrs über alle SrV-Städte angesetzt. Für Hamburg wurden Langzeitzähldaten zur Abschätzung der Radverkehrsentwicklung verwendet.

\begin{tabular}{|c|c|c|c|c|}
\hline \multirow[b]{2}{*}{ Stadt } & \multicolumn{2}{|c|}{$\begin{array}{l}\text { Verkehrsleistung im } \\
\text { Radverkehr }[\mathrm{km} / \mathrm{P}, \mathrm{d}]\end{array}$} & \multirow{2}{*}{$\begin{array}{c}\text { Gesamt- } \\
\text { entwicklung } \\
\text { Radverkehr }\end{array}$} & \multirow{2}{*}{$\begin{array}{l}\text { Jährliche } \\
\text { Entwicklung } \\
\text { Radverkehr }\end{array}$} \\
\hline & SrV2008 & SrV2013 & & \\
\hline Berlin & 1,30 & 1,60 & $+23 \%$ & $+4,6 \%$ \\
\hline Bremen & 2,50 & 2,70 & $+8 \%$ & $+1,6 \%$ \\
\hline Düsseldorf & 1,10 & 1,50 & $+36 \%$ & $+7,3 \%$ \\
\hline Halle & 1,30 & 1,20 & $-8 \%$ & $-1,5 \%$ \\
\hline Hamburg & - & - & - & $+2,1 \%$ ** \\
\hline Nürnberg & $1,36^{*}$ & $1,48^{*}$ & $+9 \%$ & $+1,8 \%$ \\
\hline Paderborn & $1,36^{*}$ & $1,48^{*}$ & $+9 \%$ & $+1,8 \%$ \\
\hline Ulm & 1,00 & 1,10 & $+10 \%$ & $+2,0 \%$ \\
\hline
\end{tabular}

** ermittelt aus Langzeitzähldaten

Tabelle 6: Abschätzung der Radverkehrsentwicklung in den betrachteten Städten

Die Ergebnisse der Unfallkenngrößen mit projizierten Radverkehrsstärken werden als das maßgebende Beurteilungskriterium angesehen und daher für alle folgenden Auswertungen verwendet.

\section{Unfallkenngrößen differenziert nach Begleitmaßnahmen}

Die auf projizierten Radverkehrsstärken beruhenden Unfallraten ergaben sich für die knotenpunktfreie Strecke nach Abbildung 31 und für Knotenpunkte nach Abbildung 32.

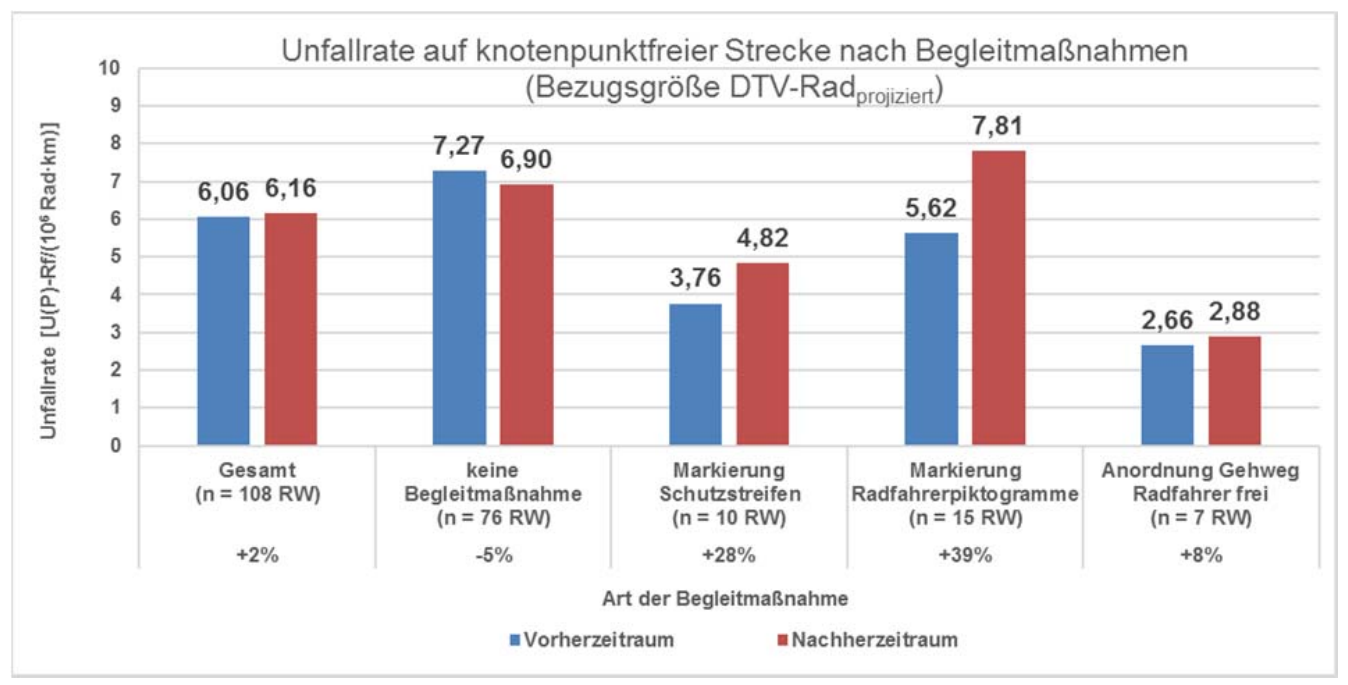

Abbildung 31: Unfallraten an knotenpunktfreier Strecke nach Begleitmaßnahmen (Bezug DTV-Rad projiziert)

Durch die Einberechnung der Radverkehrsentwicklung haben sich die Tendenzen der Unfallkenngrößen relativiert. Im Gesamtkollektiv der knotenpunktfreien Strecke ergab sich im Gegensatz zu Kapitel 5.3.1.1 nahezu ein Ausgleich der Unfallraten. An Knotenpunkten führte die Projektion der Radverkehrsstärken im Gesamtkollektiv zu einer Umkehr der Tendenzen nach Kapitel 5.3.1.1.

Vergleichbare Änderungen zeigten sich auch für Radwege ohne weitere Begleitmaßnahmen. Hier ergab sich durch die Einbeziehung der projizierten Radverkehrsstärken eine Umkehr der Tendenzen bei den Unfallraten auf der knotenpunktfreien Strecke von $+4 \%$ (ohne Projektion) zu - $5 \%$ (mit Projektion) sowie an Knotenpunkten von $+7 \%$ (ohne Projektion) zu $-2 \%$ (mit Projektion). Für die Begleitmaßnahmen Schutzstreifen, Radfahrerpiktogramme und Gehweg Radfahrer frei relativier- 
ten sich die Ergebnisse ebenfalls, jedoch haben sich die Tendenzen nach Kapitel 5.3.1.1 nicht verändert. Gleiches gilt für die Unfallkostenraten (vgl. Anhang D).

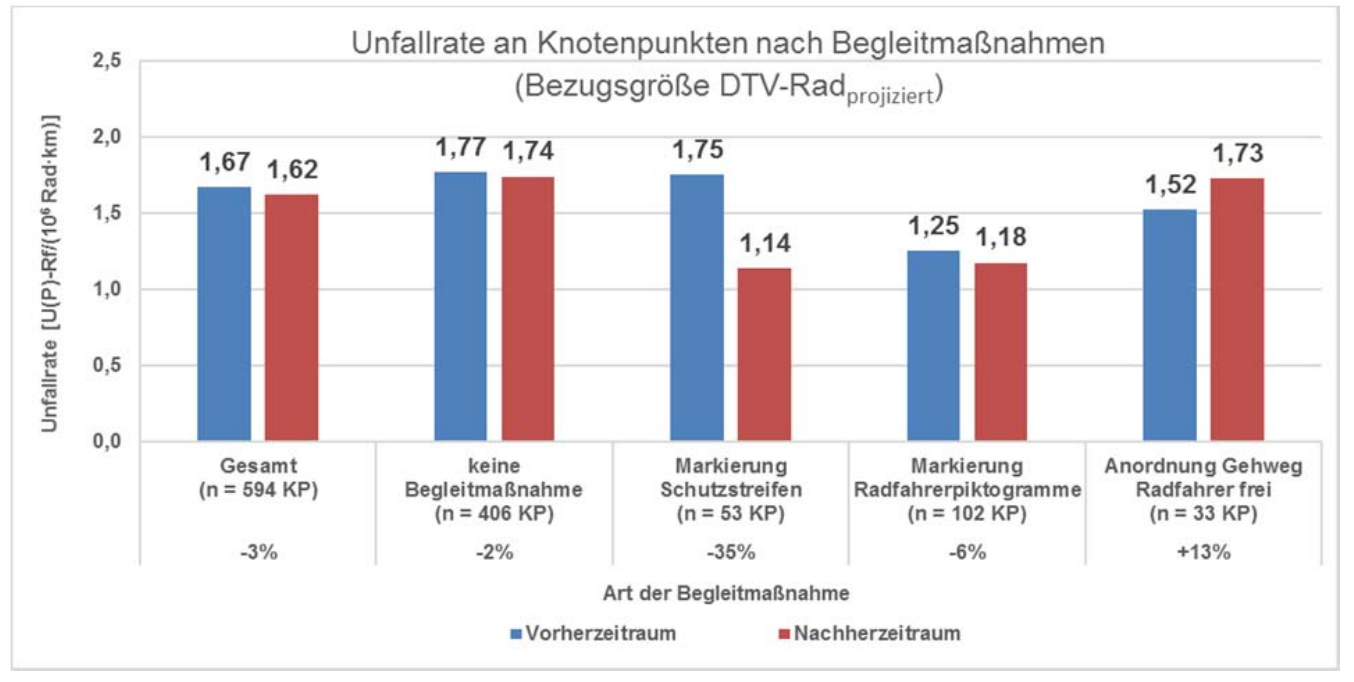

Abbildung 32: Unfallraten an Knotenpunkten nach Begleitmaßnahmen (Bezug DTV-Rad projiziert)

Zur Prüfung des Einflusses von Einzelstrecken auf die aggregierten Ergebnisse der Unfallkenngrößen wurden die Veränderungen der Unfallkenngrößen differenzierter betrachtet. Die Bewertung erfolgte hierbei über die absoluten Änderungen der Unfallkenngrößen einzelner Radwege mittels Histogrammen. Es zeigte sich, dass die Änderungen der Unfallkenngrößen einzelner Radwege einer Normalverteilung folgen. Anhand der Histogramme ließen sich dabei auch tendenziell die Ergebnisse der Unfallkenngrößen erkennen. Dies betrifft vorrangig die auf großen Stichproben basierenden Datengrundlagen (Ergebnisse auf der knotenpunktfreien Strecke bei allen Radwegen und bei Radwegen ohne Begleitmaßnahmen sowie generell bei Knotenpunkten).

Bei den Kollektiven mit kleineren Stichproben (Schutzstreifen, Radfahrerpiktogrammen und Gehweg Radfahrer frei) zeigte sich jedoch, dass die steigenden Unfallraten auf der knotenpunktfreien Strecke auf starke Erhöhungen der Unfallraten weniger Einzelstrecken zurückzuführen waren, die das Ergebnis verzerren. Ohne diese Radwege hätten sich Ergebnisse gemäß der Gesamttendenz ergeben. Die Auffälligkeit der betreffenden Radwege war dabei nicht auf besondere verkehrliche oder infrastrukturelle Merkmale zurückzuführen. Auch die unterschiedlichen Vorgehensweisen bei Schutzstreifen (mit/ohne Rückbau des Radweges) und Radfahrerpiktogrammen (Markierung auf Radweg/Fahrbahn) waren nicht ursächlich. Die Extremwerte der einzelnen Unfallraten sind zufallsbedingt und nicht auf spezifische Besonderheiten dieser Radwege zurückzuführen.

Abschließend ist anzumerken, dass die Gesamttendenz der Unfallkenngrößen maßgeblich durch das Kollektiv der nicht benutzungspflichtigen Radwege ohne weitere Begleitmaßnahmen bestimmt wird. Da hiermit auch das größte Untersuchungskollektiv (78 von 108 Radwegen mit 406 von 594 Knotenpunkten) vorliegt, erfolgt die weitere Ergebnisdarstellung nur für dieses Teilkollektiv. Die Betrachtung der kleineren Untersuchungskollektive von anderen Begleitmaßnahmen führte dagegen nicht zu belastbaren Aussagen. Deren Ergebnisse wurden zwar parallel berücksichtig jedoch nur bei nennenswerten Unterschieden bzw. Auffälligkeiten thematisiert (vgl. Kapitel 4.1.7).

\section{Unfallkenngrößen differenziert nach Kraftfahrzeugverkehrsstärken}

Der Vergleich der Unfallkenngrößen nach Kraftfahrzeugverkehrsstärken erfolgte durch Differenzierung der Kraftfahrzeugverkehrsstärke in Verbindung mit der Anzahl der Fahrstreifen gemäß den Belastungsbereichen der ERA [2010]. Für die Grenzwerte der Belastungsbereiche nach Abbildung 6 wurde mit dem Faktor 10 die Tagesverkehrsstärke abgeschätzt und das Ergebnis entsprechend 
der fahrtrichtungsfeinen Betrachtung halbiert. Für die untersuchten Hauptverkehrsstraßen mit 50 $\mathrm{km} / \mathrm{h}$ zulässiger Höchstgeschwindigkeit ergaben sich die Grenzwerte nach Tabelle 7. Die Ergebnisse für nicht benutzungspflichtige Radwege ohne weitere Begleitmaßnahmen enthält Anhang $\mathrm{E}$.

\begin{tabular}{|l|c|c|}
\hline & zweistreifige Straßen & mehrstreifige Straßen \\
\hline Belastungsbereich II & kleiner 5.000 & kleiner 8.000 \\
\hline Belastungsbereich III & $5.000-9.000$ & $8.000-11.000$ \\
\hline Belastungsbereich IV & ab 9.000 & ab 11.000 \\
\hline
\end{tabular}

Tabelle 7: Umgerechnete richtungsbezogene Tagesverkehrsstärken in Anlehnung an ERA [2010]

Die Differenzierung ergab, dass $50 \%$ der untersuchten nicht benutzungspflichtigen Radwege in Belastungsbereich II liegen, $30 \%$ in Belastungsbereich III und $20 \%$ in Belastungsbereich IV. Gemäß ERA [2010] ist in Belastungsbereich II eine Trennung von Rad- und Kraftfahrzeugverkehr durch - im Kontext dieser Untersuchung - einen benutzungspflichtigen Radweg nicht vorgesehen, in Belastungsbereich III kann dies erforderlich sein und in Belastungsbereich IV wird es gefordert. Dreiviertel der untersuchten Radwege in Belastungsbereich IV unterschreiten jedoch das Mindestmaß eines Radweges von 1,50 m gemäß VwV-StVO, was vermutlich zur Aufhebung der Benutzungspflicht führte. Die anderen Radwege in Belastungsbereich IV sind mindestens 1,60 m breit. Somit wurden bei der Aufhebung der Benutzungspflicht andere Aspekte berücksichtigt.

Auf der knotenpunktfreien Strecke mit einem Fahrstreifen je Richtung nahm die Unfallrate nach Aufhebung der Radwegebenutzungspflicht bei weniger als $5.000 \mathrm{Kfz}$ pro Tag und Richtung zu, bei Verkehrsstärken über dieser Größenordnung ab. Bei mehr als einem Fahrstreifen je Richtung ergab sich eine Erhöhung der Unfallraten unabhängig der Verkehrsstärken, welche bei Verkehrsstärken unter $8.000 \mathrm{Kfz}$ pro Tag und Richtung am stärksten war. Vergleichbare Tendenzen zeigten die Unfallraten an Knotenpunkten. Jedoch ergab sich abweichend von der knotenpunktfreien Strecke bei mehr als einem Fahrstreifen je Richtung und über $11.000 \mathrm{Kfz}$ pro Tag und Richtung ein Rückgang der Unfallrate. Die Unfallkostenraten zeigten keine einheitlichen Tendenzen.

Warum bei geringen Verkehrsstärken die Unfallraten vergleichsweise deutlich zunehmen, konnte mit dem vorliegenden Streckenkollektiv nicht abschließend beantwortet werden. Infrastrukturelle Ursachen für diese Entwicklung konnten nicht identifiziert werden. Es wurde vermutet, dass bei geringen Kraftfahrzeugverkehrsstärken mehr Radfahrer neben dem nicht benutzungspflichtigen Radweg auf der Fahrbahn fahren und dort ggf. mehr Unfälle geschehen als im Straßenseitenraum vermieden werden. Derartige Verlagerungseffekte im Unfallgeschehen zeigte die makroskopische und mikroskopische Unfallanalyse jedoch nicht (vgl. Kapitel 5.3.3).

\section{Unfallkenngrößen differenziert nach Radverkehrsstärken}

Die Differenzierung nach Radverkehrsstärken zeigt Anhang F. Die Klasseneinteilung erfolgte hier nach einer ausgeglichenen Klassenbesetzung, sodass nach Radverkehrsstärken je Fahrtrichtung unter $250 \mathrm{Rad} / 24 \mathrm{~h}$, von 250 bis $500 \mathrm{Rad} / 24 \mathrm{~h}$ und über $500 \mathrm{Rad} / 24 \mathrm{~h}$ unterschieden wurde.

Es wurde deutlich, dass auf der knotenpunktfreien Strecke bei geringen Radverkehrsstärken unter 250 Radfahrer pro Tag und Richtung die Aufhebung der Radwegebenutzungspflicht zu einer Abnahme der Unfallrate führte, wohingegen sich bei Radverkehrsstärken von 250 - 500 Radfahrer pro Tag und Richtung eine starke und bei größerem Radverkehrsaufkommen eine leichte Zunahme ergab. Es zeigten sich daher Verbesserungen der Verkehrssicherheit bei geringen Radverkehrsstärken. An Knotenpunkten ergab sich insgesamt ein Rückgang der Unfallraten, dies jedoch nur in geringen Ausprägungen (maximal -10\%). Die Unfallkostenraten nahmen auf der knoten- 
punktfreien Strecke generell ab, die Unfallschwere sinkt. An Knotenpunkten zeigte sich dies nur bei Radverkehrsstärken unter 500 Radfahrer pro Tag und Richtung.

\section{Unfallkenngrößen differenziert nach Fahrstreifenanzahl und -breite}

Die Unterscheidung nach der Anzahl der Fahrstreifen je Fahrtrichtung zeigt Anhang G sowie nach der Breite des rechten Fahrstreifens - welcher nach Aufhebung der Radwegebenutzungspflicht als Verkehrsraum für die Radfahrer möglich ist - Anhang $\mathrm{H}$. Die Klasseneinteilung für die Breiten des rechten Fahrstreifens orientiert sich an den Vorgaben der ERA [2010] gemäß Kapitel 2.3.2.

Für die Unfallraten ergab sich eine Abnahme mit Aufhebung der Radwegebenutzungspflicht bei 1 bis 2 Fahrstreifen auf der knotenpunktfreien Strecke. Bei mehr als 2 Fahrstreifen kam es dagegen zu einer Zunahme des Unfallrisikos. Die Ergebnisse der Unfallkostenrate verdeutlichten einen Rückgang der Unfallschwere unabhängig der Fahrstreifenanzahl. Bei Radunfällen an den Knotenpunkten zeigte sich nach Aufhebung der Radwegebenutzungspflicht eine Abnahme der Unfallrate nur bei mehr als 2 Fahrstreifen, bei den Unfallkostenraten ergab sich tendenziell eine Erhöhung.

Bei Fahrstreifenbreiten auf der knotenpunktfreien Strecke unter 3,00 m und über 3,50 m nahm die Unfallrate zu. Dazwischen kam es zu einer Abnahme des Unfallrisikos. Die Unfallraten an Knotenpunkten weisen vergleichbare Tendenzen auf. Bei schmalen und breiten Fahrstreifen führt die Aufhebung der Radwegebenutzungspflicht somit zu negativen Effekten. Bei den Unfallkostenraten zeigten sich nennenswerte Abnahmen bei über 3,00 m breiten Fahrstreifen auf der knotenpunktfreien Strecke sowie eine deutliche Zunahme bei 3,50 m breiten Fahrstreifen an Knotenpunkten.

\subsubsection{Fazit der Unfallkenngrößenberechnung}

Die Ergebnisse der Unfallkenngrößenberechnung - eingangs ohne Berücksichtigung der Radverkehrsentwicklung - haben differenziert nach den Begleitmaßnahmen mit Aufhebung der Radwegebenutzungspflicht erste Unterschiede innerhalb der Gesamttendenz nach Kapitel 5.1 aufgezeigt. Grundlegend stieg das Unfallrisiko für Radfahrer auf der knotenpunktfreien Strecke unabhängig der jeweiligen Begleitmaßnahmen an. Für Knotenpunkte gilt diese Entwicklung ebenfalls, jedoch ergaben sich Ausnahmen bei der Markierung von Schutzstreifen und Radfahrerpiktogrammen.

Die anschließenden Berechnungen der Unfallkenngrößen unter Berücksichtigung der jährlichen Radverkehrsentwicklung relativierten dagegen die genannten Ergebnisse. Zusammenfassend verdeutlichte diese Auswertung, dass die Erhöhung der Unfallzahlen mit der Steigerung des Radverkehrsaufkommens der vergangenen Jahre erklärt werden kann. Unter Berücksichtigung des gestiegenen Radverkehrsaufkommens zeigten sich hier keine Verschlechterungen der Verkehrssicherheit mehr.

Bei den Radwegen ohne Begleitmaßnahmen ergab sich mit Aufhebung der Benutzungspflicht nur eine vernachlässigbare Veränderung der Unfallraten sowohl auf der knotenpunktfreien Strecke als auch an Knotenpunkten. Das Unfallrisiko hat sich hier mit Aufhebung der Radwegebenutzungspflicht kaum verändert. Dagegen haben die Unfallkostenraten auf der knotenpunktfreien Strecke stark abgenommen und verweisen auf eine Verringerung der Unfallschwere.

Bei Markierungen von Schutzstreifen und Radfahrerpiktogrammen sowie der Anordnung Gehweg Radfahrer frei ergaben sich dagegen für die knotenpunktfreie Strecke vergleichsweise deutliche Erhöhungen der Unfallraten. Die Zunahme der Unfallzahlen konnten hier demzufolge nicht mit der Radverkehrsentwicklung erklärt werden. Diese Tendenzen waren jedoch lediglich aufgrund besonderer Unfallbelastungen von einzelnen Radwegen zustande gekommen und sind dagegen nicht als generelle Entwicklung zu werten. Ursächlich hierfür können auch die geringen Stichprobengrö- 
ßen bei diesen Begleitmaßnahmen sein, weswegen nachfolgend hauptsächlich das größte Untersuchungskollektiv der Radwege ohne Begleitmaßnahmen dargestellt wurde. Argumentationen zu den anderen Begleitmaßnahmen erfolgten dagegen nur bei besonderen Auffälligkeiten.

Für das Kollektiv der Radwege ohne Begleitmaßnahmen erfolgte eine weitere Differenzierung nach den Belastungsbereichen der ERA (Kraftfahrzeugverkehrsstärke und Fahrstreifenanzahl). Hier befanden sich $50 \%$ der Radwege in Belastungsbereichen, für welche die ERA eine getrennte Führung von Rad- und Mischverkehr als sinnvoll erachten bzw. diese fordern. Die Radwegegebenutzungspflicht wurde an diesen Radwegen vermutlich vorrangig aufgrund nicht eingehaltener Mindestbreiten nach VwV-StVO aufgehoben.

Insgesamt ergab sich ein höheres Unfallrisiko für Radfahrer auf der knotenpunktfreien Strecke und an Knotenpunkten nach Aufhebung der Radwegebenutzungspflicht im Belastungsbereich II (Verkehrsstärken unter $5.000 \mathrm{Kfz}$ pro Tag und einem Fahrstreifen je Richtung sowie Verkehrsstärken unter $8.000 \mathrm{Kfz}$ pro Tag und mehr als einem Fahrstreifen je Richtung). Infrastrukturelle Gründe für diese Entwicklungen konnten jedoch mit dem zugrundeliegenden Streckenkollektiv nicht ermittelt werden. Die bei geringen Verkehrsstärken vermuteten Verlagerungseffekte im Unfallgeschehen auf die Fahrbahn aufgrund entsprechender Änderungen in der Flächennutzung bestätigten die nachfolgenden Analysen jedoch nicht (vgl. Kapitel 5.3.3).

Bei der Differenzierung nach Radverkehrsstärken wurde deutlich, dass sich mit Aufhebung der Radwegebenutzungspflicht negative Effekte auf die Verkehrssicherheit der knotenpunktfreien Strecke einstellen, wenn auf den Streckenabschnitten mehr als 250 Radfahrer pro Tag und Richtung unterwegs sind. Außerdem zeigten die Unfallkenngrößen der knotenpunktfreien Strecke negative Effekte nach Aufhebung der Radwegebenutzungspflicht, wenn mehr als 2 Fahrstreifen am Streckenabschnitt vorhanden sind. Die im Rahmen der Auswertungen nach Fahrstreifenbreiten aufgezeigten Problembereiche finden sich bei Breiten unter 3,00 m sowie über 3,50 m. Ob sich hier tatsächlich negative Auswirkungen durch die Aufhebung der Radwegebenutzungspflicht und ein verändertes Fahrverhalten oder verlagertes Unfallgeschehen ergeben haben, wird jedoch erst nach den weiteren Untersuchungsschritten abschließend diskutiert. 


\subsubsection{Exkurs: Flächennutzung der Radfahrer an nicht benutzungspflichtigen Radwegen}

An dieser Stelle erfolgt ein Exkurs zu den Ergebnissen aus den eigenen Verkehrszählungen. Die Erhebungen wurden an Radwegen ohne vorhandene Zähldaten durchgeführt, welche zur Unfallkenngrößenberechnung benötigt wurden. Hierbei wurde gleichzeitig nach der tatsächlichen Flächennutzung der Radfahrer unterschieden. Die Ergebnisse bezüglich der Flächennutzung der Radfahrer differenziert nach den betrachteten Begleitmaßnahmen enthält Abbildung 33.

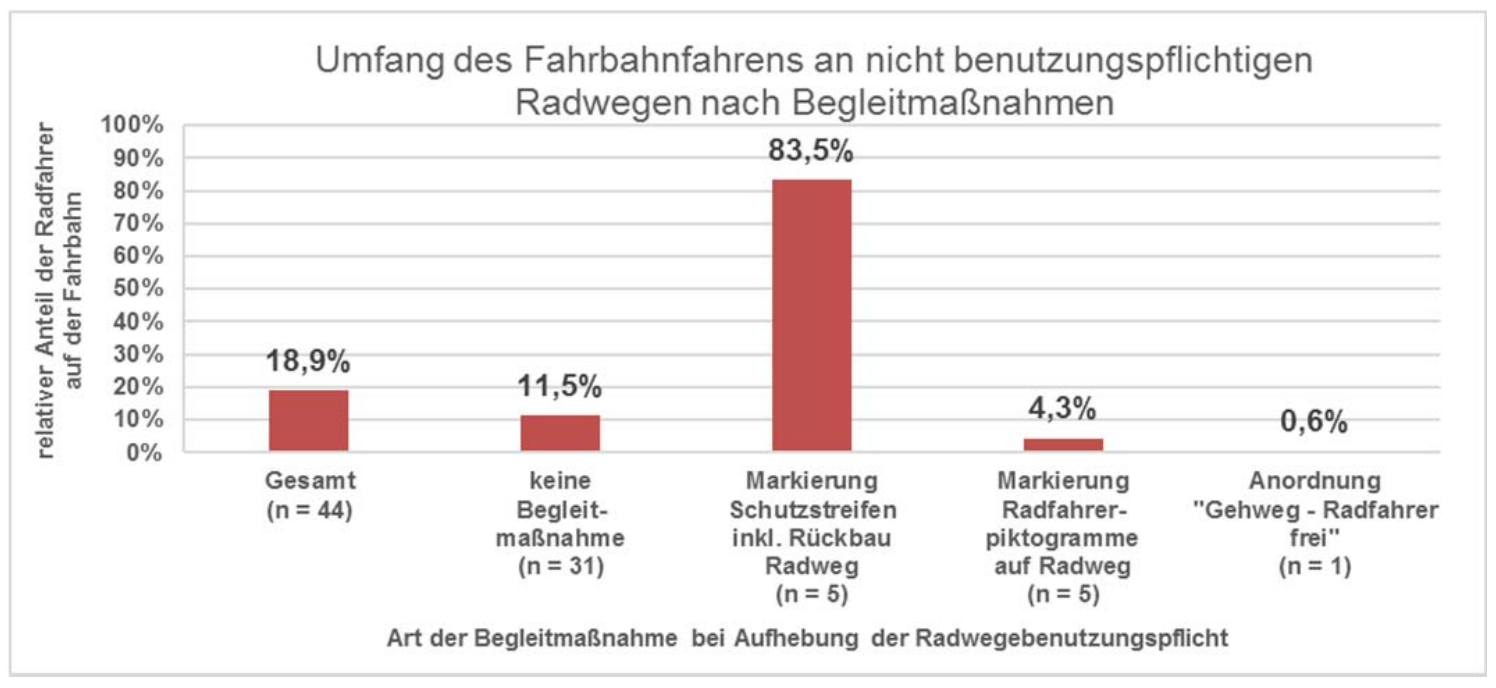

\section{Abbildung 33: Nutzung der Fahrbahn von Radfahrern an nicht benutzungspflichtigen Radwegen}

In der Gesamtbetrachtung zeigte sich mit nur 18,9\% eine insgesamt geringe Nutzung der Fahrbahn neben nicht benutzungspflichtigen Radwegen. Außerdem traten erhebliche Unterschiede zwischen den einzelnen Begleitmaßnahmen mit Aufhebung der Radwegebenutzungspflicht auf. Im größten Kollektiv der nicht benutzungspflichtigen Radwege ohne weitere Begleitmaßnahmen fuhren im Mittel nur knapp $12 \%$ der Radfahrer auf der Fahrbahn. Durch diese Werte konnten die Ergebnisse der Flächennutzung nach Alrutz et al. [2009] bestätigt werden (vgl. Kapitel 2.5.2). An nicht benutzungspflichtigen Radwegen mit markierten Radfahrerpiktogrammen auf dem Radweg sowie der Anordnung Gehweg Radfahrer frei lag die Fahrbahnnutzung in noch geringerem Ausmaß vor. Die Ergebnisse basieren hier jedoch nur auf einzelnen Radverkehrsanlagen.

Einzig für die Markierung von Schutzstreifen bei gleichzeitigem Rückbau der Radwege fuhr die große Mehrheit der Radfahrer auf der Fahrbahn (83,5\%). Der verbleibende Anteil der Radfahrer fuhr entsprechend regelwidrig auf dem Gehweg im Seitenraum. Auch mit diesen Ergebnissen konnten die Flächennutzungen für diese Art der Radverkehrsanlage nach Alrutz et al. [2009] bestätigt werden. Bei der dualen Führung von Schutzstreifen und nicht benutzungspflichtigen Radwegen liegt nach Landeshauptstadt München [2016] die Nutzung des Schutzstreifens dagegen nur bei ca. $20 \%$. Derartige Radwege waren jedoch im Kollektiv der Radwege mit erfolgten eigenen Zählungen nicht enthalten, so dass hierzu im Projekt keine Aussagen getroffen werden konnten.

Für das größte Kollektiv der nicht benutzungspflichtigen Radwege ohne Begleitmaßnahmen $(n=31)$ erfolgte eine Analyse der Flächennutzung nach weiteren verkehrlichen und infrastrukturellen Merkmalen. Bei der Betrachtung von verkehrlichen Randbedingungen zeigte sich ein Rückgang der Fahrbahnnutzung durch die Radfahrer mit steigendem Verkehrsaufkommen im Kraftfahrzeug- und Schwerverkehr (Bestimmtheitsmaße $\mathrm{R}^{2}=0,14$ bzw. $\mathrm{R}^{2}=0,27$, vgl. Abbildung 34). Demzufolge führen höhere Verkehrsstärken zu einem tendenziellen Verbleib der Radfahrer auf dem nicht benutzungspflichtigen separaten Radweg. Grundlegend war dabei zu beachten, dass auch 
zwischen den Kraftfahrzeug- und Schwerverkehrsstärken starke innere Zusammenhänge vorhanden sind. Hier ergab sich ein steigendes Schwerverkehrsaufkommen mit steigendem Kraftfahrzeugverkehr mit einem Bestimmtheitsmaß von $\mathrm{R}^{2}=0,76$. Abhängigkeiten zwischen dem Fahrbahnfahren und dem Radverkehrsaufkommen konnten in den Analysen dagegen nicht festgestellt werden.
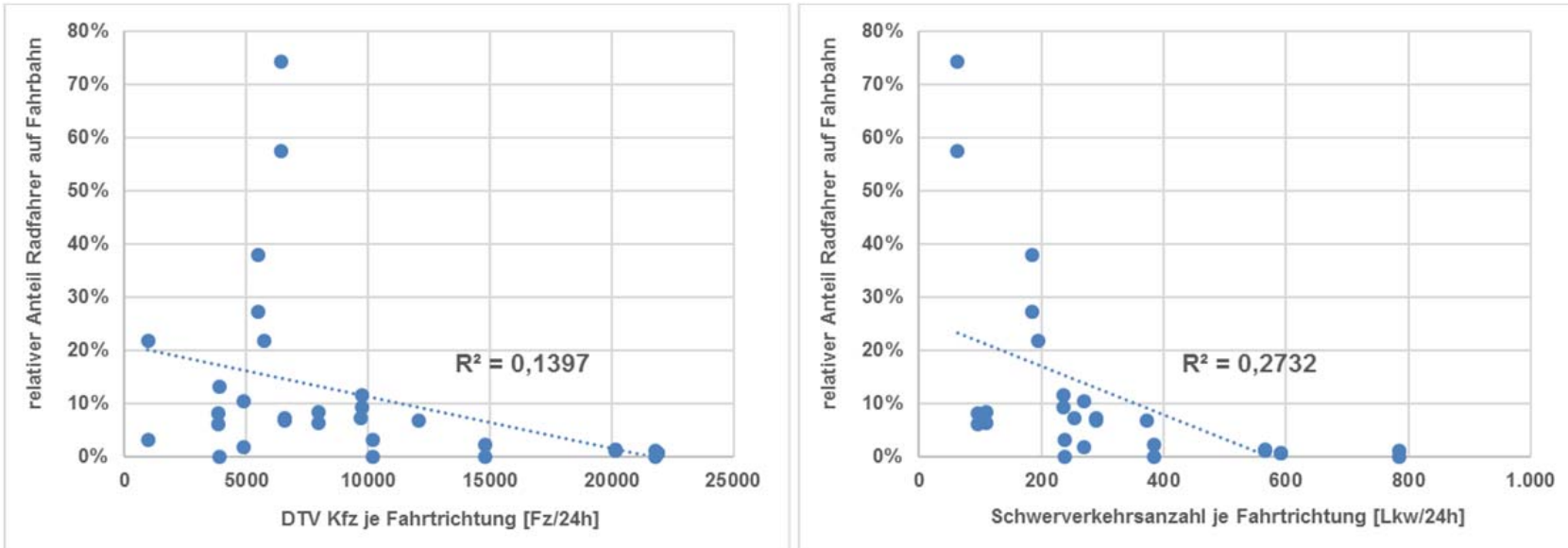

\section{Abbildung 34: Nutzung der Fahrbahn von Radfahrern in Abhängigkeit des Kraftfahrzeugverkehrs an nicht benutzungspflichtigen Radwegen ohne weitere Begleitmaßnahmen $(n=31)$}

Auffallend sind in Abbildung 34 zudem die 6 Radwege mit über 20 \% Fahrbahnnutzung. Ursache dieses hohen Anteils des Fahrbahnfahrens waren die infrastrukturellen Merkmale der Radwege. Alle 6 Radwege sind mit unter 1,00 m deutlich zu schmal, die angrenzenden Fahrstreifen dagegen mit über 4,50 m überbreit. Da diese Kombination von Querschnittselementen bei den anderen 25 Radwegen mit Fahrbahnnutzung unter $20 \%$ nicht auftrat, sind hier die breiten Fahrstreifen und schmalen Radwege ausschlaggebend für den hohen Anteil der Fahrbahnnutzung.

Ein entsprechendes Ergebnis zeigte sich auch bei Betrachtung der Querschnittselemente aller 31 Radwege. Hier ergab sich eine Zunahme des Fahrbahnfahrens der Radfahrer mit steigender Breite des rechten Fahrstreifens $\left(R^{2}=0,37\right)$ sowie ein Rückgang der Fahrbahnnutzung mit zunehmender Breite des nicht benutzungspflichtigen Radweges $\left(R^{2}=0,10\right)$. Somit wurden bei ausreichend breiten Verkehrsräumen auf der Fahrbahn diese auch in größerem Umfang genutzt. Lag dagegen ein ausreichend breiter nicht benutzungspflichtiger Radweg vor, so führte dies auch in größerem Umfang zu einer Weiternutzung dieser Radverkehrsanlage und zur Meidung der Fahrbahn. 


\subsubsection{Ergebnisse der makroskopischen und mikroskopischen Unfallanalyse}

Dieses Kapitel enthält die Ergebnisse der gemeinsamen makroskopischen und mikroskopischen Unfallanalyse. Dabei werden vorrangig die Verschiebungen des Radunfallgeschehens in der Flächennutzung mit Aufhebung der Radwegebenutzungspflicht beschrieben. Nach der Differenzierung nach Begleitmaßnahmen erfolgt die Auswertung bezüglich der allgemeinen Unfallmerkmale dabei nur für Radwege ohne weitere Begleitmaßnahmen (vgl. Kapitel 4.1.7).

\subsubsection{Radunfallgeschehen nach Begleitmaßnahmen}

Die Ergebnisse differenziert nach Begleitmaßnahmen und Streckenelementen (knotenpunktfreie Strecke und Knotenpunkte) enthält Anhang I. Insgesamt ergab sich eine Zunahme der Radunfälle, welche nach Kapitel 5.3 auf die Radverkehrsentwicklung zurückzuführen war. Die Verkehrssicherheit des Radverkehrs hat sich nach Aufhebung der Radwegebenutzungspflicht daher nicht wesentlich verändert. Hinsichtlich der Flächennutzung des am Unfall beteiligten Radfahrers zeigten sich für Radwege ohne weitere Begleitmaßnahmen mit Aufhebung der Radwegebenutzungspflicht marginale Verschiebungen der Radunfälle auf die Fahrbahn sowohl für Strecken- als auch für Knotenpunktunfälle. Unter Berücksichtigung des geringen Anteils des Fahrbahnfahrens an nicht benutzungspflichtigen Radwegen (vgl. Kapitel 5.3.2) waren jedoch auch keine starken Verschiebungen zu erwarten. Bei der Anordnung Gehweg Radfahrer frei zeigte sich dagegen eine stärkere Verlagerung des Radunfallgeschehens auf die Fahrbahn. Aufgrund der fehlenden Daten zur tatsächlichen Flächennutzung der Radfahrer nach Aufhebung der Benutzungspflicht auf diesen Strecken, war eine abschließende Bewertung dieser Verschiebung im Unfallgeschehen nicht möglich.

Es war weiterhin zu beachten, dass bereits bei noch bestehender Radwegebenutzungspflicht vergleichsweise häufig Radunfälle auf der Fahrbahn geschahen. Dieser hohe Anteil der Fahrbahnunfälle vor Aufhebung der Radwegebenutzungspflicht war in allen untersuchten Städten in einer ähnlichen Größenordnung vorhanden und nicht mit bestimmten Charakteristiken erklärbar. Daher mussten diese Unfälle auf eine regelwidrige Fahrbahnnutzung der Radfahrer vor den Änderungen zur Radwegebenutzungspflicht zurückgeführt werden. Für Radwege mit der Markierung von Schutzstreifen und Radfahrerpiktogrammen als Begleitmaßnahme konnte aufgrund fehlender mikroskopischer Unfalldaten nicht nach der Flächennutzung der Radfahrer unterschieden werden.

\subsubsection{Radunfallgeschehen an Radwegen ohne Begleitmaßnahmen nach allgemeinen Un- fallmerkmalen}

Die nachfolgenden Ergebnisse beziehen sich ausschließlich auf Radwege ohne weitere Begleitmaßnahmen mit Aufhebung der Radwegbenutzungspflicht. Die parallel durchgeführten Betrachtungen der anderen Begleitmaßnahmen ließen aufgrund der geringen Unfallzahlen und der Differenzierung nach weiteren Unfallmerkmalen oftmals keine aussagekräftigen Schlussfolgerungen zu.

\section{Unfallkategorien}

Beim Vergleich der Verteilung der Unfallkategorien (Anhang J) war zu erkennen, dass die Unfallschwere mit Aufhebung der Radwegebenutzungspflicht auf der knotenpunktfreien Strecke deutlich zurückging, sich an den Knotenpunkten jedoch insgesamt leicht erhöhte.

Bei der Differenzierung nach der Flächennutzung der unfallbeteiligten Radfahrer kam es bei Unfällen mit Leichtverletzten auf der knotenpunktfreien Strecke und an Knotenpunkten zu einer geringfügigen Verlagerung der Radunfälle auf die Fahrbahn. Bei Unfällen mit Schwerverletzten ergab sich dagegen eine tendenzielle Verschiebung der Radunfälle in den Seitenraum. 


\section{Unfalltypen}

Die Ergebnisse der Unfalltypenverteilung enthält Anhang K. In der Gesamtbetrachtung der knotenpunktfreien Strecke wurde deutlich, dass hier vergleichsweise viele Abbiege- und Einbiegen/Kreuzen-Unfälle auftraten, welche normalerweise vorrangig an Knotenpunkten geschehen. Dabei handelte es sich ausschließlich um Unfälle an Grundstückszufahren. Insgesamt haben sich mit Aufhebung der Radwegebenutzungspflicht auch bestimmte Verschiebungen zwischen den Unfalltypen eingestellt. Der überdurchschnittlichen Zunahme der Abbiegeunfälle steht hier eine überdurchschnittliche Abnahme der Einbiegen-/Kreuzen-Unfälle entgegen.

Bezüglich der Flächennutzung betrafen nach Abbildung 35 weitere Verschiebungen die Einbiegen/Kreuzen-Unfälle mit tendenziell mehr Radunfällen auf der Fahrbahn. Bei Unfällen im Längsverkehr ergab sich eine Verschiebung in ähnlicher Größenordnung in den Seitenraum. Die weiteren Unfalltypen waren bezüglich Verschiebungen in der Flächennutzung unauffällig.

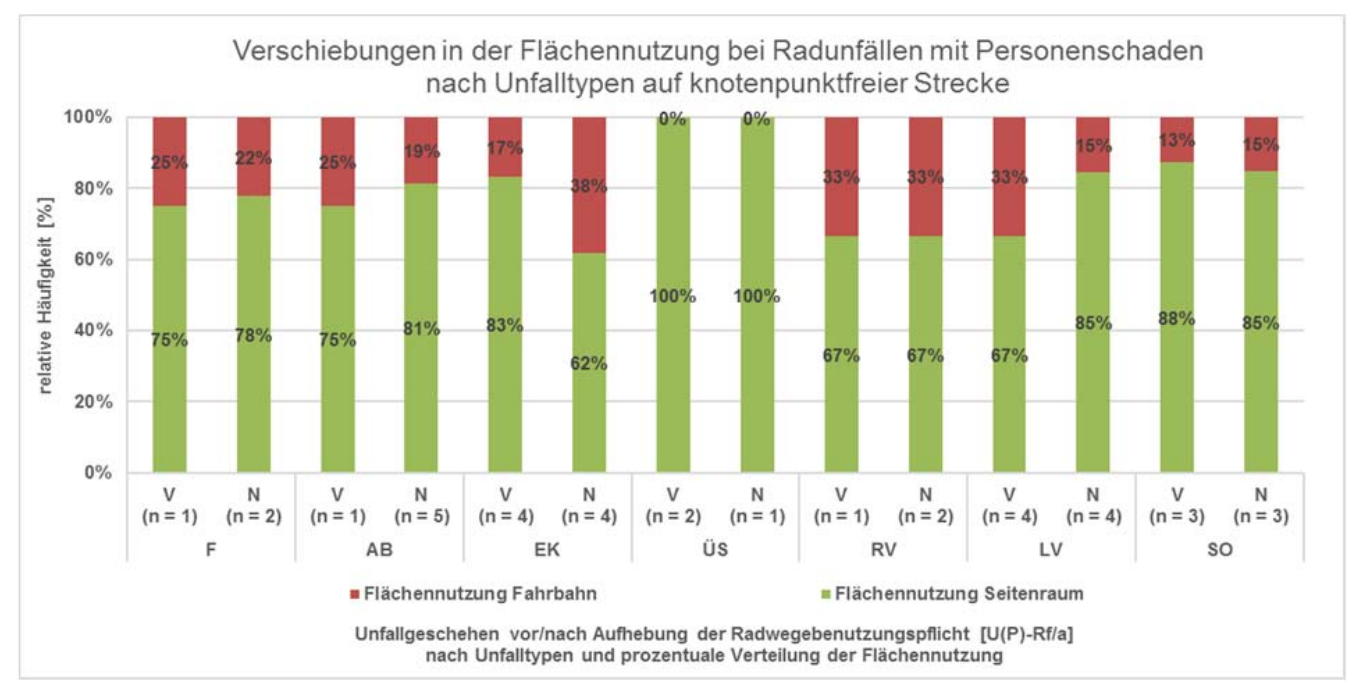

\section{Abbildung 35: Verschiebungen in der Flächennutzung bei Radunfällen auf der knotenpunktfreien Strecke nach Unfalltypen}

Bei den Radunfällen an Knotenpunkten konnte auf ein deutlich größeres Unfallkollektiv zurückgegriffen werden. Insgesamt waren hier mit knapp $80 \%$ die Abbiegen- und Einbiegen-/KreuzenUnfälle maßgebend (vgl. Anhang K). Beim Vergleich der Situation vor und nach Aufhebung der Radwegebenutzungspflicht zeigte sich, dass Abbiegeunfälle entgegen der Gesamttendenz leicht rückläufig waren. Der Anteil der Rechtsabbiegeunfälle lag hier unabhängig der Radwegebenutzungspflicht bei etwa $80 \%$. Bei den Einbiegen-/Kreuzen-Unfälle ergab sich dagegen eine überdurchschnittliche Zunahme von $+20 \%$ im Vergleich zur Gesamtunfallentwicklung.

Die Verschiebungen bezüglich der Flächennutzung vor und nach Aufhebung der Radwegbenutzungspflicht enthält Abbildung 36. Hier kam es bei den Abbiegeunfällen zu einer geringfügigen Verlagerung der Unfälle auf die Fahrbahn. Die wesentlichen Verschiebungen sind dabei bei den Rechtsabbiegeunfällen aufgetreten. Vor Aufhebung der Radwegebenutzungspflicht geschahen $66 \%$ dieser Unfälle mit Radfahrern im Seitenraum, nach Aufhebung der Radwegebenutzungspflicht waren es $62 \%$ auf der Fahrbahn, was auf entsprechende Verlagerungseffekte hinweist. Auch bei den Linksabbiegeunfällen war eine Verschiebung der Unfälle auf die Fahrbahn erkennbar. Bei den Einbiegen-/Kreuzen-Unfällen wurden keine Verschiebungen in der Flächennutzung festgestellt. Abschließend zeigten sich Verschiebungen der Radunfälle im Längsverkehr in den Seitenraum, die verbleibenden Unfalltypen waren dagegen unauffällig. 


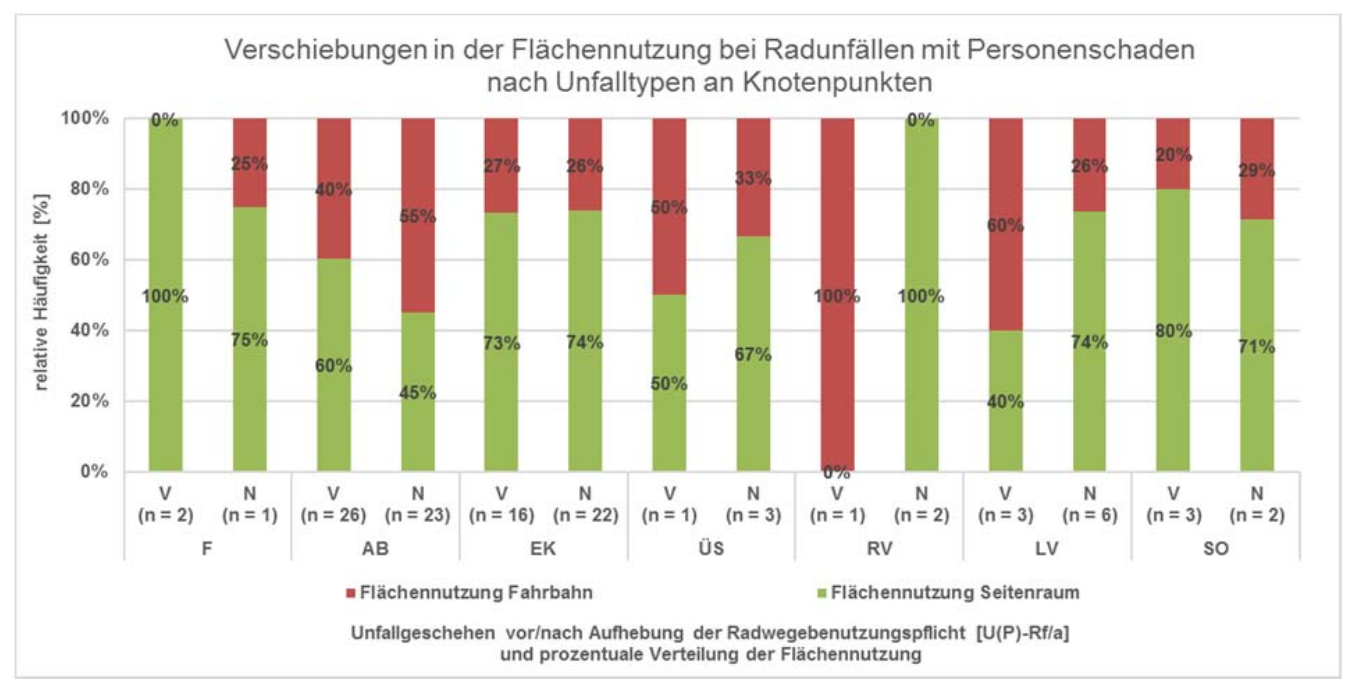

\section{Abbildung 36: Verschiebungen in der Flächennutzung bei Radunfällen an Knotenpunkten nach Unfalltypen}

\section{Unfallarten}

Die durchgeführte Analyse der Unfallarten verdeutlichte, dass größtenteils Probleme im Radunfallgeschehen vorliegen, wenn sich Verkehrsströme kreuzen. Dies betrifft Unfälle auf der knotenpunktfreien Strecke als auch an Knotenpunkten gleichermaßen. Insgesamt konnte die Auswertung der Unfallarten nur die vorherigen Ergebnisse nach den Unfalltypen bestätigen.

\section{Äußere Randbedingungen (Straßen- und Lichtverhältnisse)}

Bei der Differenzierung nach Straßenverhältnissen waren Unfälle bei Trockenheit maßgebend aber hinsichtlich der Flächennutzung unauffällig. Geringfügige Verschiebungen des Unfallgeschehens in den Seitenraum nach Aufhebung der Radwegebenutzungspflicht zeigten sich nur bei Nässe.

Bezüglich der Lichtverhältnisse bilden Unfälle bei Tageslicht das größte Teilkollektiv. Bei der Flächennutzung an Knotenpunkten ergaben sich marginale Verschiebungen, wenn bei Tageslicht auf der Fahrbahn sowie bei Dämmerung und Dunkelheit im Seitenraum gefahren wird.

\section{Verknüpfung Flächennutzung in Unfallgeschehen und Verkehrsverhalten}

Die Zusammenhänge zwischen Fahrbahnnutzung und Radunfallgeschehen auf der Fahrbahn wurden für die Radwege ohne Benutzungspflicht (ohne Begleitmaßnahmen) mit eigenen Zähldaten (vgl. Kapitel 5.3.2) geprüft. Dabei erfolgte eine Differenzierung nach den vorhandenen Kraftfahrzeugverkehrsstärken. Für Radwege mit angrenzenden Kraftfahrzeugverkehrsstärken je Richtung unter $5.000 \mathrm{Kfz} / 24 \mathrm{~h}$ lagen keine Unfallhergangsbeschreibungen vor. Daher konnten nur 23 Radwege in die Ergebnisse nach Abbildung 37 einfließen (gültig nur für knotenpunktfreie Strecke).

Die Auswertung hat gezeigt, dass mit zunehmender Kraftfahrzeugverkehrsstärke neben den nicht benutzungspflichtigen Radwegen sowohl der Anteil der Radfahrer auf der Fahrbahn als auch der Anteil der Radunfälle auf der Fahrbahn zurückgehen. Des Weiteren übersteigt der Anteil der Radunfälle auf der Fahrbahn den Anteil der dort tatsächlich fahrendenden Radfahrer. Dies zeigte sich besonders bei Kraftfahrzeugverkehrsstärken von mehr als $10.000 \mathrm{Kfz}$ pro Tag und Richtung. Hier geschahen $18 \%$ der Radunfälle auf der Fahrbahn, wobei diese Fläche nur $1 \%$ der Radfahrer nutzten. Die deutet auf ein höheres Risiko für Radunfälle auf der Fahrbahn hin. Unter Berücksichtigung der geringen Unfallzahlen waren jedoch tiefergehende Interpretationen und spätere Empfehlungen nicht möglich. Die hier genannten Ergebnisse sind nur als Indizien anzusehen, dagegen nicht als belastbare Zusammenhänge. 

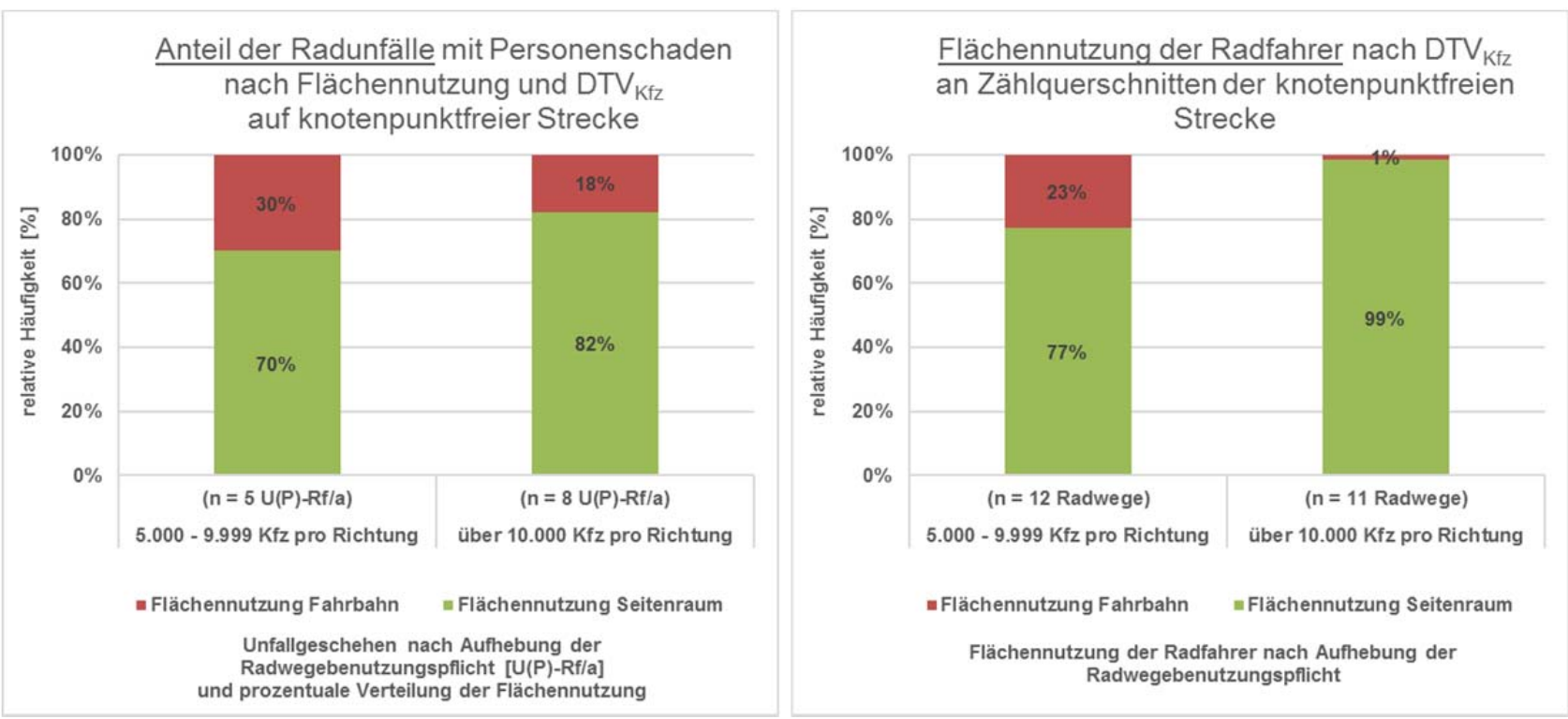

Abbildung 37: Unfallgeschehen und Fahrverhalten von Radfahrern auf der knotenpunktfreien Strecke differenziert nach Flächennutzung und Kraftfahrzeugverkehrsstärken

\subsubsection{Radunfallgeschehen unter Berücksichtigung der Unfallbeteiligten}

Nach den allgemeinen Unfallmerkmalen enthält dieses Kapitel die Ergebnisse nach den Unfallbeteiligten. Dabei wurde nach dem Alter, dem Geschlecht, der Art der Verkehrsbeteiligung und den Unfallursachen sowie nach der jeweiligen Beteiligung des Radfahrers am Unfall unterschieden.

\section{Hauptunfallverursacher}

Die Ergebnisse der Hauptverursacher von Radunfällen enthält Anhang L. Es zeigte sich, dass auf der knotenpunktfreien Strecke $56 \%$ und an Knotenpunkten $67 \%$ der Unfälle nicht vom Radfahrer verursacht wurden. Dies war wesentlich auf die Vielzahl der Abbiegen- und Einbiegen-/KreuzenUnfälle zurückzuführen, bei denen in der Regel nicht der Radfahrer der Hauptverursacher ist.

Zudem war festzustellen, dass nach Aufhebung der Radwegebenutzungspflicht tendenziell die Radfahrer als Hauptunfallverurscher zurückgingen und andere Verkehrsteilnehmer als Hauptunfallverursacher zunahmen. Es wurde vermutet, dass nach Aufhebung der Radwegebenutzungspflicht keine Unfallschuld für Radfahrer mehr durch regelwidriges Radfahren auf der Fahrbahn vermerkt wird. Dies deutete sich auch in den Ergebnissen zur Flächennutzung an. Die späteren Auswertungen der Unfallursachen konnten diese Vermutung jedoch nicht bestätigten.

\section{Alter der Hauptunfallverursacher}

Bei den Auswertungen nach dem Alter wurden 6 Altersklassen unterschieden und ebenfalls nach dem Hauptunfallverursacher differenziert. In den Ergebnissen der einzelnen Altersgruppen konnten jedoch keine nennenswerten Tendenzen und hinsichtlich der Flächennutzung keine relevanten Verschiebungen festgestellt werden. Aufgrund der oftmals kleinen Teilunfallkollektive waren zudem keine tieferen Interpretationen möglich.

Es war jedoch zu hinterfragen, welche Auswirkungen die Aufhebung der Radwegebenutzungspflicht auf das Unfallgeschehen einzelner Altersgruppen haben soll. Es wurde vermutet, dass ggf. bei jüngeren Radfahrern eine umfangreichere Fahrbahnnutzung auftritt, wohingegen bei älteren Verkehrsteilnehmern das subjektive Sicherheitsempfinden beim Fahren im Seitenraum höher ist und der nicht benutzungspflichtige Radweg vermehrt weitergenutzt wird. Im Unfallgeschehen haben sich derartige Verschiebungen in den jeweiligen Teilunfallkollektiven jedoch nicht ergeben. 


\section{Geschlecht der Hauptunfallverursacher}

In den Ergebnissen nach dem Geschlecht der Hauptunfallverursacher zeigte sich unabhängig der weiteren Differenzierungen, dass männliche Verkehrsteilnehmer vorrangig Hauptunfallverursacher an den betrachteten Radwegen gewesen sind. Insgesamt haben sich jedoch bei den Auswertungen zum Geschlecht keine weiteren neuen Erkenntnisse ergeben. Nennenswerte Verschiebungen in der Flächennutzung blieben ebenfalls aus.

Bezüglich des Geschlechtes waren jedoch die gleichen Aspekte zu hinterfragen, wie bereits bei den Auswertungen zu den Altersgruppen. Aussagekräftigere Ergebnisse zu Veränderungen im Unfallgeschehen mit Aufhebung der Radwegebenutzungspflicht werden dagegen von den nachfolgenden Verkehrsbeteiligungen der Hauptunfallverursacher erwartet.

\section{Art der Verkehrsbeteiligung}

Insgesamt wurde bei fremdverschuldeten Radunfällen deutlich, dass es sich bei den entsprechenden Hauptunfallverursachern vordergründig um Pkw handelte (72\% auf knotenpunktfreier Strecke, $81 \%$ an Knotenpunkten). Bei der Flächennutzung ergaben sich kaum Verschiebungen des Radunfallgeschehens nach Aufhebung der Radwegebenutzungspflicht auf die Fahrbahn. Aufgrund der teilweise geringen Unfallzahlen erfolgten keine weiteren Auswertungen in den anderen Teilunfallkollektiven.

Bei Betrachtung der Radfahrer als Hauptunfallverursacher wies der Kraftfahrzeugverkehr als Unfallgegner auf der knotenpunktfreien Strecke mit $17 \%$ nur einen geringen Stellenwert auf. Von Radfahrern verursachte Unfälle mit Kraftfahrzeugen sind auf der knotenpunktfreien Strecke daher kein Unfallschwerpunkt. Stattdessen steigt hier die Bedeutung der nicht motorisierten Unfallgegner. Dies waren vorrangig Unfälle mit Fahrrädern untereinander, Unfälle mit Fußgängern und Alleinunfälle. Bei den Alleinunfällen ist aber auch die erhebliche Dunkelziffer zu berücksichtigen, welche nach Below [2016] etwa $90 \%$ beträgt. Hinsichtlich der Flächennutzung waren aufgrund der geringen Unfallzahlen keine weiteren Aussagen zu Verschiebungen möglich.

An den Knotenpunkten traten dagegen Pkw als Unfallgegner (Radfahrer Hauptunfallverursacher) mit $41 \%$ erneut in den Vordergrund. Nennenswerten Unterschiede in der Flächennutzung zeigten sich jedoch für dieses Unfallkollektiv nicht. Differenzierte Auswertungen der nicht motorisierten Unfallgegner nach der Flächennutzung waren aufgrund der geringen Unfallzahlen weiterhin nicht möglich.

\section{Unfallursachen}

Waren an Unfällen mit Radverkehrsbeteiligung auf der knotenpunktfreien Strecke nicht die Radfahrer der Hauptunfallverursacher, so waren als Unfallursachen mit knapp $60 \%$ die Fehler beim Abbiegen, Wenden oder Rückwärtsfahren maßgebend. In Bezug zur Flächennutzung ergab sich für diese Unfälle eine marginale Verschiebung auf die Fahrbahn. Als zweite relevante Unfallursache zeigte sich das falsche Verhalten von Fußgängern. Hierfür sind allerdings keine wesentlichen Veränderungen mit Aufhebung der Radwegebenutzungspflicht erkennbar geworden. Alle weiteren Unfallursachen haben nur eine untergeordnete Bedeutung.

Bei den von anderen Verkehrsteilnehmern verursachten Radunfällen an Knotenpunkten traten neben den Fehlern beim Abbiegen, Wenden oder Rückwärtsfahren mit $61 \%$ als zweite maßgebliche Unfallursache die Vorfahrtsverletzungen mit insgesamt 23\% auf. Hinsichtlich der Flächennutzung zeigte sich für diese beiden maßgebenden Unfallursachen eine nur geringfügige Verschiebung zu mehr Unfällen auf die Fahrbahn. 
Waren auf der knotenpunktfreien Strecke die Radfahrer der Hauptunfallverursacher, so war neben den sonstigen Ursachen die Unfallursache „Fehler Straßenbenutzung“ auffällig. Der vermutete Rückgang des regelwidrigen Fahrbahnfahrens als Unfallursache mit Aufhebung der Radwegebenutzungspflicht konnte jedoch bestätigt werden. Relevante Verschiebungen in der Flächennutzung haben sich ebenfalls nicht eingestellt. Die weiteren Unfallursachen waren aufgrund der nur geringen Kollektivgrößen nur von untergeordneter Bedeutung.

Für die Knotenpunkte waren abschließend etwas größere Unfallkollektive bei den Ursachen der unfallverursachenden Radfahrer auswertbar. Maßgebend waren hier Fehler beim Abbiegen, Wenden oder Rückwärtsfahren, Vorfahrt- und Vorrangverletzungen und „Fehler Straßenbenutzung“. Verschiebungen bezüglich der Flächennutzung zeigten sich dabei nicht. Bei den verbleibenden Unfallursachen lag auch hier kein höherer Stellenwert vor. Abschließend kann bezüglich der Unfallursachen festgehalten werden, dass durch diese Auswertungen im Wesentlichen nur die Ergebnisse vorheriger Auswerteschritte bestätigt werden konnten.

\subsubsection{Fazit der makroskopischen und mikroskopischen Unfallanalyse}

Insgesamt hat die gemeinsame makroskopische und mikroskopische Unfallanalyse die folgenden wesentlichen Erkenntnisse sowie entsprechende Schlussfolgerungen bezüglich der Verkehrssicherheit mit Aufhebung der Radwegebenutzungspflicht hervorgebracht.

Bei der Differenzierung nach Begleitmaßnahmen ergaben sich die bereits aus Kapitel 5.3 .1 bekannten Zusammenhänge. Unter Berücksichtigung der Radverkehrsentwicklung konnten an Radwegen ohne weitere Begleitmaßnahmen mit Aufhebung der Radwegebenutzungspflicht somit keine Veränderungen der Verkehrssicherheit festgestellt werden. Zudem hat die makroskopische und mikroskopische Unfallanalyse gezeigt, dass sich kaum Verschiebungen des Radunfallgeschehens auf die Fahrbahn ergeben haben. Hierbei war aber auch zu beachten, dass bereits bei noch bestehender Radwegebenutzungspflicht ein nicht unerheblicher Anteil der Radunfälle auf der Fahrbahn geschehen ist, was auf eine regelwidrige Flächennutzung hinwies.

Mit Aufhebung der Radwegebenutzungspflicht (ohne weitere Begleitmaßnahmen) hat sich die Unfallschwere auf der knotenpunktfreien Strecke deutlich verringert, jedoch hat sich diese an Knotenpunkten leicht erhöht. Bei Betrachtung der Unfalltypen hat sich gezeigt, dass Probleme mit dem Radverkehr nach Aufhebung der Radwegebenutzungspflicht an Grundstückszufahrten auf der knotenpunktfreien Strecke sowie auch an Knotenpunkten bestehen. Dabei sind überwiegend Abbiegeunfälle und Einbiegen-/Kreuzen-Unfälle im Vordergrund. Zudem haben sich mit Aufhebung der Radwegebenutzungspflicht geringfügige Verschiebungen zwischen den Unfalltypen eingestellt. Diese können aufgrund der ausgebliebenen Verhaltensänderungen jedoch nicht mit der Aufhebung der Radwegebenutzungspflicht erklärt werden. Verschiebungen in der Flächennutzung der unfallbeteiligten Radfahrer waren daher ebenfalls kaum feststellbar.

Die Ergebnisse der Auswertungen zu den Unfallarten bestätigten die bisherigen Aussagen zu den Unfalltypen. Nennenswerte Effekte der Straßen- und Lichtverhältnisse zeigten sich nicht. Der Vergleich von Flächennutzung und Unfallgeschehen ergab für die knotenpunktfreie Strecke, dass der Anteil der Radunfälle auf der Fahrbahn den Anteil der dort fahrenden Radfahrer übersteigt. Hierbei war jedoch aber auch die geringe Datengrundlage zu beachten, sodass die genannten Erkenntnisse nur als Indizien angesehen werden konnten und keine belastbaren Zusammenhänge darstellen. Für Knotenpunkte waren hier keine Aussagen möglich.

Bei Betrachtung der Hauptunfallverursacher haben sich Verschiebungen von den Radfahrern hin zu den anderen Verkehrsteilnehmern ergeben. Hinsichtlich Alter und Geschlecht blieben nen- 
nenswerte Effekte im Unfallgeschehen mit Aufhebung der Radwegebenutzungspflicht aus. Pkw bilden den wesentlichen Schwerpunkt der Unfallverursacher bei fremdverschuldeten Radunfällen (Art der Verkehrsbeteiligung). Waren dagegen Radfahrer die Unfallverursacher, so traten die nichtmotorisierten Verkehrsteilnehmer (Fußgänger, andere Radfahrer oder Alleinunfälle) stärker in den Vordergrund. Anhand der Unfallursachen wurden abschließend nur bereits bekannte Zusammenhänge bestätigt. Nennenswerte Verschiebungen in der Flächennutzung sind in den Auswertungen zu den einzelnen Unfallbeteiligten nicht aufgetreten.

Insgesamt kann festgehalten werden, dass nach den Erkenntnissen der Unfallkenngrößenberechnung, welche keine wesentlichen Änderungen im Radunfallgeschehen aufzeigten, sich in der gemeinsamen makroskopischen und mikroskopischen Unfallanalyse kaum Verschiebungen zwischen einzelnen Unfallmerkmalen sowie in der tatsächlichen Flächennutzung des Radfahrers ergeben haben. Wesentliche strukturelle Änderungen im Unfallgeschehen konnten im Rahmen der Unfallanalysen für nicht benutzungspflichtige Radwege ohne weitere Begleitmaßnahmen nicht nachgewiesen werden. Deutlich positive oder negative Effekte bezüglich der Verkehrssicherheit des Radverkehrs als Gesamtheit können der Aufhebung der Radwegebenutzungspflicht somit nicht zugeschrieben werden.

Abschließend konnten durch die vorrangige Weiternutzung der nicht benutzungspflichtigen Radwege (siehe Exkurs Flächennutzung in Kapitel 5.3.2) auch keine strukturellen Veränderungen im Radunfallgeschehen erwartet werden. Somit gilt es den tatsächlichen Nutzen der Aufhebung der Radwegebenutzungspflicht für die Verkehrssicherheit und generell den Radverkehr zu hinterfragen. 


\section{Ergebnisse der Verkehrsverhaltensanalyse}

Eine Übersicht der Ergebnisse der Verkehrsverhaltensanalyse zeigt Tabelle 8. Insgesamt wurden an Radwegen mit Benutzungspflicht 7.317 Radfahrer mit 82 Konflikten sowie an Radwegen ohne Benutzungspflicht 8.436 Radfahrer mit 420 Konflikten beobachtet. Ein direkter Vergleich des Konfliktgeschehens von Radwegen mit und ohne Benutzungspflicht in der Gesamtheit ist jedoch aufgrund der unterschiedlichen Bewertungskonzepte nach Kapitel 4.3.2 (u.a. direkter VorherNachher-Vergleich vs. alternativer Mit-Ohne-Vergleich) nicht zulässig.

\begin{tabular}{|c|c|c|c|c|}
\cline { 2 - 5 } \multicolumn{1}{c|}{} & \multicolumn{2}{c|}{ Radwege mit Benutzungspflicht } & \multicolumn{2}{c|}{ Radwege ohne Benutzungspflicht } \\
\cline { 2 - 5 } & im Seitenraum & auf Fahrbahn & im Seitenraum & auf Fahrbahn \\
\hline $\begin{array}{c}\text { beobachtete } \\
\text { Radfahrer }\end{array}$ & 7.128 & 189 & 7.847 & 589 \\
\hline $\begin{array}{c}\text { leichte } \\
\text { Konflikte }\end{array}$ & 70 & 4 & 395 & 7 \\
\hline $\begin{array}{c}\text { schwere } \\
\text { Konflikte }\end{array}$ & 8 & 0 & 18 & 0 \\
\hline
\end{tabular}

Tabelle 8: Ergebnisübersicht zur Verkehrsverhaltensanalyse

Die direkte Vergleichbarkeit der Konflikte an Radwegen mit und ohne Benutzungspflicht war nur für die 2 Radwege im Vorher-Nachher-Vergleich gegeben, da es sich um die gleichen Streckenabschnitte mit identischer Streckencharakteristik handelte. Für den Großteil der untersuchten Radwege konnten jedoch nur Mit-Ohne-Vergleiche realisiert werden. Doch auch wenn bei der Auswahl der Vergleichsstrecken auf eine ähnliche Streckencharakteristik geachtet wurde, traten hier durch streckenspezifische Singularitäten erhebliche Unterschiede im Konfliktgeschehen auf (z.B. Bushaltestelle mit Vielzahl von Haltestellenkonflikten). Die Erklärung der Unterschiede im Konfliktgeschehen basierend auf der Radwegebenutzungspflicht war für diese Radwege nicht möglich. Das Konfliktgeschehen der Radwege mit und ohne Benutzungspflicht wurde daher nur getrennt für sich betrachtet, ohne Vergleiche anzustellen. Nachfolgend werden in Kapitel 6.1 die Erkenntnisse zur Flächennutzung und in Kapitel 6.2 zum Konfliktgeschehen der Radfahrer dargestellt.

\subsection{Erkenntnisse zur Flächennutzung}

\subsubsection{Radverkehrsanlagen der Vorher-Nachher-Vergleiche}

Bei der Strecke mit der Markierung eines Radfahrstreifens (inkl. Rückbau des Radweges) als Begleitmaßnahme handelte es sich insgesamt um einen sehr schwach belasteten Streckenabschnitt. Hinsichtlich der Flächennutzung ergaben sich die Ergebnisse nach Tabelle 9.

\begin{tabular}{|c|c|c|c|c|c|}
\hline \multicolumn{5}{|c|}{ Flächennutzung Vorher-Szenario } \\
\hline \multicolumn{2}{|c|}{ Radweg } & \multicolumn{2}{c|}{ Fahrbahn } & \multicolumn{2}{c|}{ Gehweg } \\
\hline $\begin{array}{c}\text { in Fahrt- } \\
\text { richtung }\end{array}$ & Linksfahrer & $\begin{array}{c}\text { in Fahrt- } \\
\text { richtung }\end{array}$ & Linksfahrer & $\begin{array}{c}\text { in Fahrt- } \\
\text { richtung }\end{array}$ & Linksfahrer \\
\hline 75 & 7 & - & - & 5 & 1 \\
\hline $\mathbf{8 5 \%}$ & $\mathbf{8 \%}$ & - & - & $\mathbf{6 \%}$ & $\mathbf{1} \%$ \\
\hline
\end{tabular}

\begin{tabular}{|c|c|c|c|c|c|}
\hline \multicolumn{6}{|c|}{ Flächennutzung Nachher-Szenario } \\
\hline \multicolumn{2}{|c|}{ Radweg } & \multicolumn{2}{c|}{ Fahrbahn } & \multicolumn{2}{c|}{ Gehweg } \\
\hline $\begin{array}{c}\text { in Fahrt- } \\
\text { richtung }\end{array}$ & Linksfahrer & $\begin{array}{c}\text { in Fahrt- } \\
\text { richtung }\end{array}$ & Linksfahrer & $\begin{array}{c}\text { in Fahrt- } \\
\text { richtung }\end{array}$ & Linksfahrer \\
\hline- & - & 64 & - & 28 & 7 \\
\hline- & - & $\mathbf{6 5 \%}$ & - & $\mathbf{2 8 \%}$ & $\mathbf{7 \%}$ \\
\hline
\end{tabular}

Tabelle 9: Flächennutzung vor und nach Aufhebung der Radwegebenutzungspflicht bei der Begleitmaßnahme Radfahrstreifen und Rückbau Radweg

Den ursprünglichen benutzungspflichtigen Radweg haben (unabhängig der Fahrtrichtung) insgesamt $93 \%$ der Radfahrer genutzt. Die verbleibenden Radfahrer nutzten regelwidrig den Gehweg. Nach Markierung des - wiederum benutzungspflichtigen - Radfahrstreifens und Rückbau des ursprünglichen Radweges fuhren 65 \% der Radfahrer auf der Fahrbahn. Die anderen 35 \% der Rad- 
fahrer fuhren dagegen regelwidrig auf dem Gehweg. Somit ergaben sich hier deutliche Verlagerungseffekte, da eigene Verkehrsflächen für Radfahrer auf der Fahrbahn geschaffen wurden.

Bei der Strecke mit der Markierung von Radfahrerpiktogrammen auf dem nicht benutzungspflichtigen Radweg und am Fahrbahnrand als Begleitmaßnahme handelte es sich ebenfalls um eine schwach belastete Radverkehrsverbindung. Die Flächennutzung zeigt Tabelle 10.

\begin{tabular}{|c|c|c|c|c|c|}
\hline \multicolumn{6}{|c|}{ Flächennutzung Vorher-Szenario } \\
\hline \multicolumn{2}{|c|}{ Radweg } & \multicolumn{2}{c|}{ Fahrbahn } & \multicolumn{2}{c|}{ Gehweg } \\
\hline $\begin{array}{c}\text { in Fahrt- } \\
\text { richtung }\end{array}$ & Linksfahrer & $\begin{array}{c}\text { in Fahrt- } \\
\text { richtung }\end{array}$ & Linksfahrer & $\begin{array}{c}\text { in Fahrt- } \\
\text { richtung }\end{array}$ & Linksfahrer \\
\hline 279 & 30 & 14 & 0 & 18 & 3 \\
\hline $\mathbf{8 1 \%}$ & $\mathbf{9 \%}$ & $\mathbf{4 \%}$ & $\mathbf{0} \%$ & $\mathbf{5 \%}$ & $\mathbf{1 \%}$ \\
\hline
\end{tabular}

\begin{tabular}{|c|c|c|c|c|c|}
\hline \multicolumn{5}{|c|}{ Flächennutzung Nachher-Szenario } \\
\hline \multicolumn{2}{|c|}{ Radweg } & \multicolumn{2}{c|}{ Fahrbahn } & \multicolumn{2}{c|}{ Gehweg } \\
\hline $\begin{array}{c}\text { in Fahrt- } \\
\text { richtung }\end{array}$ & Linksfahrer & $\begin{array}{c}\text { in Fahrt- } \\
\text { richtung }\end{array}$ & Linksfahrer & $\begin{array}{c}\text { in Fahrt- } \\
\text { richtung }\end{array}$ & Linksfahrer \\
\hline 185 & 31 & 13 & 0 & 13 & 6 \\
\hline $\mathbf{7 5 \%}$ & $\mathbf{1 3 \%}$ & $\mathbf{5 \%}$ & $\mathbf{0 \%}$ & $\mathbf{5 \%}$ & $\mathbf{2 \%}$ \\
\hline
\end{tabular}

Tabelle 10: Flächennutzung vor und nach Aufhebung der Radwegebenutzungspflicht bei der Begleitmaßnahme Radfahrerpiktogramme

Der Radweg wurde von etwa $90 \%$ der Radfahrer - unabhängig der Radwegebenutzungspflicht und Fahrtrichtung - genutzt. Die regelwidrige Gehwegnutzung trat zu etwa $7 \%$, die Fahrbahnnutzung zu etwa $5 \%$ auf, dies unabhängig der Radwegebenutzungspflicht. Insgesamt zeigte sich, dass es mit Aufhebung der Radwegebenutzungspflicht zu keiner Veränderung in der Flächennutzung kam. Verlagerungseffekte der Radfahrer wurden nicht festgestellt.

\subsubsection{Radverkehrsanlagen der Mit-Ohne-Vergleiche}

Die aggregierten Ergebnisse zur Flächennutzung an den 4 Radwegen mit ähnlicher Streckencharakteristik differenziert nach der Radwegebenutzungspflicht enthält Tabelle 11. Die Aufhebung der Radwegebenutzungspflicht erfolgte an allen 4 Vergleichsstrecken ohne Begleitmaßnahmen.

\begin{tabular}{|c|c|c|c|c|c|}
\hline \multicolumn{5}{|c|}{ Flächennutzung mit Radwegebenutzungspflicht } \\
\hline \multicolumn{2}{|c|}{ Radweg } & \multicolumn{2}{c|}{ Fahrbahn } & \multicolumn{2}{c|}{ Gehweg } \\
\hline $\begin{array}{c}\text { in Fahrt- } \\
\text { richtung }\end{array}$ & Linksfahrer & $\begin{array}{c}\text { in Fahrt- } \\
\text { richtung }\end{array}$ & Linksfahrer & $\begin{array}{c}\text { in Fahrt- } \\
\text { richtung }\end{array}$ & Linksfahrer \\
\hline 5.138 & 104 & 151 & - & 72 & 140 \\
\hline $\mathbf{9 2 \%}$ & $\mathbf{2 \%}$ & $\mathbf{3 \%}$ & - & $\mathbf{1 \%}$ & $\mathbf{2 \%}$ \\
\hline
\end{tabular}

\begin{tabular}{|c|c|c|c|c|c|}
\hline \multicolumn{5}{|c|}{ Flächennutzung ohne Radwegebenutzungspflicht } \\
\hline \multicolumn{2}{|c|}{ Radweg } & \multicolumn{2}{c|}{ Fahrbahn } & \multicolumn{2}{c|}{ Gehweg } \\
\hline $\begin{array}{c}\text { in Fahrt- } \\
\text { richtung }\end{array}$ & Linksfahrer & $\begin{array}{c}\text { in Fahrt- } \\
\text { richtung }\end{array}$ & Linksfahrer & $\begin{array}{c}\text { in Fahrt- } \\
\text { richtung }\end{array}$ & Linksfahrer \\
\hline 4.560 & 67 & 33 & - & 69 & 112 \\
\hline $\mathbf{9 4 \%}$ & $\mathbf{1 \%}$ & $\mathbf{1 \%}$ & - & $\mathbf{1 \%}$ & $\mathbf{2} \%$ \\
\hline
\end{tabular}

Tabelle 11: Flächennutzung an 4 Radwegen mit ähnlicher Streckencharakteristik differenziert nach Radwegebenutzungspflicht (Aufhebung ohne weitere Begleitmaßnahmen)

Bei dieser Auswertung konnten die bereits bekannten Erkenntnisse (vgl. Kapitel 5.3.2 und Kapitel 6.1.1) mit einer umfangreicheren Datenbasis bestätigt werden. Entsprechend zeigte sich unabhängig der Radwegebenutzungspflicht eine deutlich untergeordnete Fahrbahnnutzung. Nicht benutzungspflichtige Radwege ohne Begleitmaßnahmen werden somit umfassend weitergenutzt. Eine Differenzierung nach dem - im Rahmen der Unfallanalyse hinterfragten - Geschlecht und Alter der Radfahrer ergab zudem, dass es tendenziell männliche Radfahrer sind, welche die Fahrbahn nutzen. Zudem fährt fast ausschließlich die Altersgruppe von 18 und 65 Jahren auf der Fahrbahn.

Abschließend bestätigten auch die Vergleiche der Flächennutzung an den 4 Radwegen mit Benutzungspflicht (ohne Vergleichsstrecken) mit den 4 betrachteten nicht benutzungspflichtigen Radwegen aus der Unfallanalyse die fast ausschließliche Radwegnutzung. 


\subsection{Erkenntnisse der Konfliktanalyse}

\subsubsection{Ergebnisübersicht}

Bei den Konfliktanalysen an den zwei Radwegen mit direktem Vorher-Nachher-Vergleich waren aufgrund des geringen Radverkehrsaufkommens insgesamt nur 12 Konflikte feststellbar. Unterschiede nach der Radwegebenutzungspflicht ergaben sich hier nicht. Entsprechend der hohen Radwegnutzung geschahen auch alle Konflikte beim Fahren im Seitenraum.

Nachfolgend wird das Konfliktgeschehen aller benutzungspflichtigen und nicht benutzungspflichtigen Radwege jeweils für sich beschrieben. Vergleiche beider Szenarien sind dabei unzulässig (vgl. einleitende Anmerkungen zu Kapitel 6). Die Ergebnisse des direkten Vorher-Nachher-Vergleiches wurden hierbei ebenfalls berücksichtigt.

Eine Differenzierung des Konfliktgeschehens nach Streckenelementen und Radwegebenutzungspflicht zeigt Tabelle 12. Insgesamt geschahen $77 \%$ der Konflikte an Knotenpunkten, $19 \%$ auf der knotenpunktfreien Strecke und die verbleibenden $4 \%$ an Haltestellen. Den Schwerpunkt bilden somit Knotenpunkte, was die Ergebnisse der Unfallanalyse nach Kapitel 5.1 bestätigt.

\begin{tabular}{|c|c|c|c|}
\hline & \multirow{3}{*}{$\begin{array}{c}\begin{array}{c}\text { Radwege mit } \\
\text { Benutzungspflicht }\end{array} \\
64\end{array}$} & \multirow{3}{*}{$\begin{array}{c}\begin{array}{c}\text { Radwege ohne } \\
\text { Benutzungspflicht }\end{array} \\
321\end{array}$} \\
\hline & & & \\
\hline$\stackrel{\square}{-}$ & Konfliktanzahl & & \\
\hline 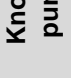 & $\begin{array}{l}\text { Konfliktanzahl (Anteil) } \\
\text { im Seitenraum }\end{array}$ & 60 (94\%) & 316 (98\%) \\
\hline \multirow{2}{*}{ 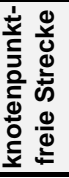 } & Konfliktanzahl & 15 & 82 \\
\hline & $\begin{array}{l}\text { Konfliktanzahl (Anteil) } \\
\text { im Seitenraum }\end{array}$ & 15 (100\%) & 80 (98\%) \\
\hline \multirow{2}{*}{ 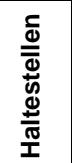 } & Konfliktanzahl & 3 & 17 \\
\hline & $\begin{array}{l}\text { Konfliktanzahl (Anteil) } \\
\text { im Seitenraum }\end{array}$ & $3(100 \%)$ & 17 (100\%) \\
\hline
\end{tabular}

Tabelle 12: Ergebnisübersicht zum Konfliktgeschehen nach Streckenelementen

Zudem wurden - entsprechend der seltenen Fahrbahnnutzung - kaum Konflikte auf der Fahrbahn festgestellt. Insgesamt wurden auf der Fahrbahn nur 11 Konflikte beobachtet. Da offenbar allein der Status der Radwegebenutzungspflicht keinen Einfluss auf die Flächennutzung und das Konfliktgeschehen hat, wurden nachfolgend die Problembereiche im Konfliktgeschehen unabhängig der Radwegebenutzungspflicht nur nach der Flächennutzung differenziert.

\subsubsection{Problembereiche im Konfliktgeschehen}

Beim Radwegfahren an Knotenpunkten entstanden von den festgestellten 376 Konflikten knapp $60 \%$ beim Einbiegen (vgl. Abbildung 38). Der maßgebende Fall war hier, dass Fahrzeuge beim Einbiegen oder beim Herstellen einer Sichtachse zur Hauptfahrbahn die Radwegefurt blockierten und Radfahrer entsprechend ausweichen mussten. Die hohe Anzahl dieses Konflikttyps im Vergleich zu den anderen Konflikttypen war damit zu erklären, dass bei einer länger blockierten Radwegefurt auch mehrere Radfahrer betroffen sein konnten.

Der mit etwa 20 \% zweithäufigste Konflikttyp entstand durch Fußgängerquerungen. Das Problem war hier ausschließlich das unachtsame Überschreiten des Radweges bzw. das Warten auf dem Radweg im Knotenpunktbereich und somit die Behinderung der bevorrechtigten Radfahrer. $16 \%$ 
der Konflikte wurden beim Rechtsabbiegen festgestellt. Dabei handelt es sich vorrangig um das Blockieren der Radwegfurt beim Rechtsabbiegen und das Übersehen der von hinten nahenden Radfahrer im Seitenraum. Alle weiteren Konflikttypen waren nur von untergeordneter Bedeutung.

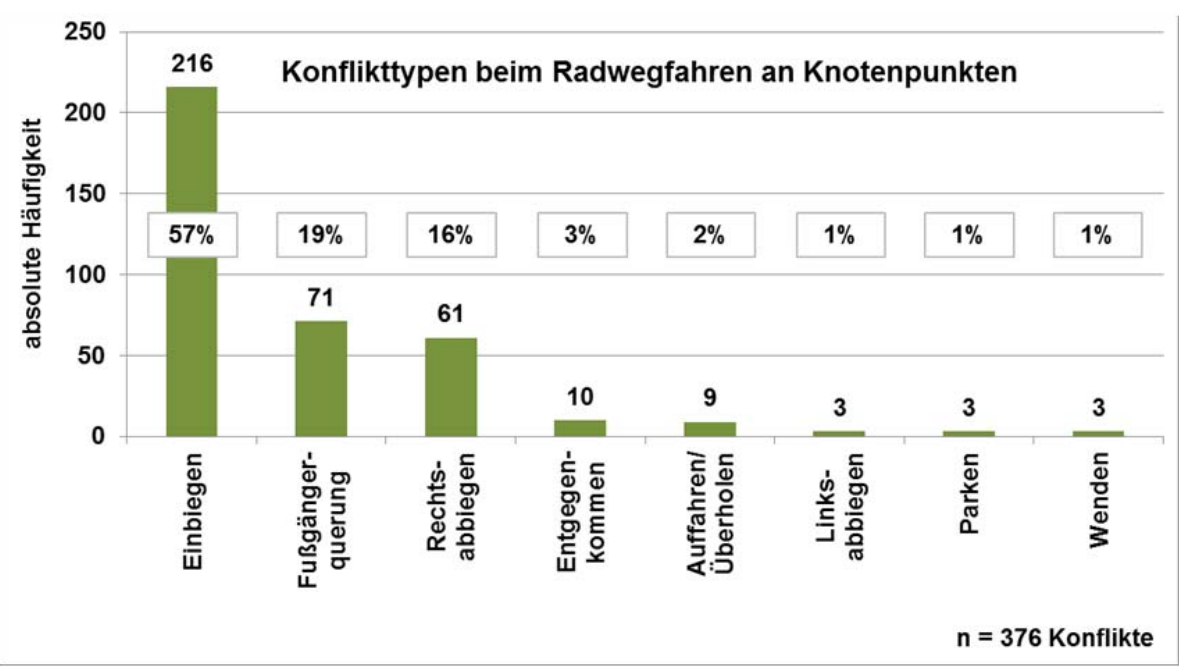

Abbildung 38: Konfliktgeschehen beim Radwegfahren an Knotenpunkten

Bei den Konflikten beim Radwegfahren auf der knotenpunktfreien Strecke zeigte sich in Abbildung 39 ein heterogeneres Ergebnis. Hier entstanden $24 \%$ der Konflikte beim Parken in Grundstückszufahrten oder Hauseingängen quer zur Fahrtrichtung auf dem Radweg, nicht dagegen an Längsparkständen neben dem Radweg. Ebenfalls zu $24 \%$ entstanden Konflikte auf dem Radweg durch Entgegenkommende, diese überwiegend hervorgerufen durch linksfahrende Radfahrer. Weitere $23 \%$ der Konflikte entstanden durch Fußgängerquerungen, welche vorrangig auf Unachtsamkeit beim Überschreiten des Radweges zurückzuführen waren. Alle weiteren Konflikte fanden dagegen nur vereinzelt statt. Hierzu zählen Konflikte mit Hindernissen auf dem Radweg (beim Beladen von Fahrzeugen am Fahrbahnrand), Konflikte im Längsverkehr (beim Überholen) sowie Abbiegen und Einbiegen in bzw. aus Grundstückszufahrten.

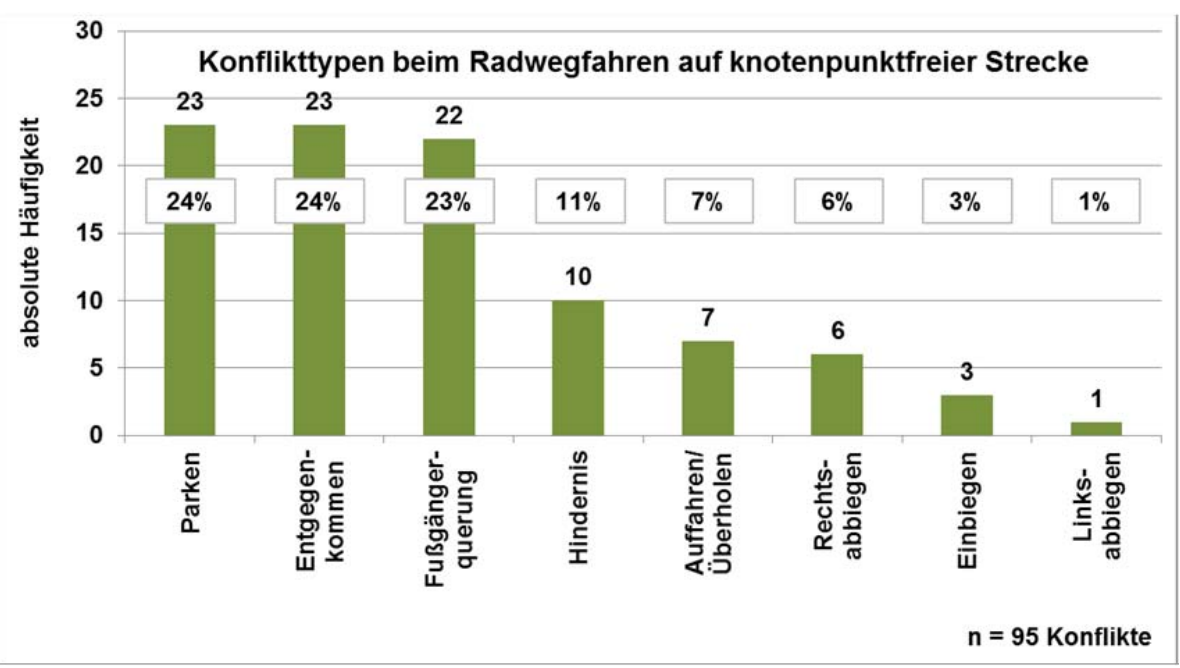

Abbildung 39: Konfliktgeschehen beim Radwegfahren auf knotenpunktfreier Strecke

Alle 20 festgestellten Konflikte beim Radwegfahren an Haltestellen wurden durch Fußgängerquerungen bzw. das Warten auf dem Radweg verursacht. Im Wesentlichen handelte es sich hierbei um Situationen, in denen Fahrgäste den Radweg bereits vor Ankunft des Busses betraten und Warteflächen fehlten. 
Bei den wenigen festgestellten Konflikten beim Fahrbahnfahren $(n=11)$ handelte es sich überwiegend um Abbiegen- und Einbiegen-Konflikte an Knotenpunkten. Weiterhin sind Konflikte beim Parken im Längsverkehr am Fahrbahnrand und Konflikte beim Wenden auf der knotenpunktfreien Strecke aufgetreten.

Als Besonderheit im Verkehrsverhalten hat sich an 2 Radwegen gezeigt, dass aufgrund von Stau auf der angrenzenden Fahrbahn, der Radweg häufig durch motorisierte Zweiräder genutzt wurde. In den 6-stündigen Erhebungen wurden auf beiden Radwegen 24 motorisierte Zweiräder beobachtet. Dabei handelte es sich zum Teil um kleine Motorroller, jedoch wurden auch zahlreiche große Motorräder festgestellt. Konflikte entstanden hierbei nicht.

Neben den bisherigen Analysen konnte für die $\mathbf{4}$ nicht benutzungspflichtigen Radwege aus der Unfallanalyse keine Erklärung für die starke Unfallzunahme mit Aufhebung der Radwegebenutzungspflicht geliefert werden. Ursachen der besonderen Veränderung der Unfallsituation waren in den Verkehrsverhaltensanalysen nicht erkennbar.

\subsection{Fazit der Verkehrsverhaltensanalyse}

In Bezug zur Flächennutzung ist festzuhalten, dass sich mit Aufhebung der Radwegebenutzungspflicht bei keinen oder nur geringfügigen Begleitmaßnahmen (z.B. Piktogrammen) keine Änderungen im Verkehrsverhalten eingestellt haben. Deutliche Verlagerungseffekte auf die Fahrbahn zeigten sich nur, wenn Begleitmaßnahmen zur Förderung des Fahrbahnfahrens (z.B. Radfahrstreifen inkl. Radwegrückbau) umgesetzt wurden. Dies bestätigte die Ergebnisse aus Kapitel 5.3.2.

Im Rahmen der Konfliktanalysen ergaben sich Schwerpunkte im Konfliktgeschehen an Knotenpunkten beim Einbiegen-/Kreuzen, Abbiegen und bei Fußgängerquerungen sowie auf der knotenpunktfreien Strecke beim Parken auf dem Radweg, durch linksfahrende Radfahrer und erneut bei Fußgängerquerungen. Bei den wenigen beobachtbaren Konflikten auf der Fahrbahn waren ebenfalls die Einbiegen-/Kreuzen-/Abbiegen-Konflikte maßgebend. Die dargestellten Konflikttypen bestätigten somit die maßgebenden Unfalltypen der Unfallanalyse.

In Hinblick auf die Radwegebenutzungspflicht ist festzuhalten, dass allein durch deren Aufhebung keine Konflikte vermieden werden. Die Verkehrsverhaltensanalysen haben gezeigt, dass durch die vorrangige Weiternutzung von nicht benutzungspflichtigen Radwegen ohne Begleitmaßnahmen auch die Konflikte weiterhin im Seitenraum geschehen. Durch ausbleibende Verhaltensänderungen in der Flächennutzung können somit auch keine Änderungen im Unfall- und Konfliktgeschehen mit Aufhebung der Radwegebenutzungspflicht auftreten. 


\section{Ergebnisse der Vor-Ort-Befragungen}

Insgesamt konnten bei den durchgeführten Befragungen 731 Verkehrsteilnehmer zu deren Verkehrsverhalten und Sicherheitsbewertung am entsprechenden Radweg befragt werden. Eine Übersicht der auswertbaren Fragebögen enthält Tabelle 13.

\begin{tabular}{|c|c|c|}
\cline { 2 - 3 } \multicolumn{1}{c|}{} & Radwege mit Benutzungspflicht & Radwege ohne Benutzungspflicht \\
\hline Radfahrer & 258 & 321 \\
\hline Fußgänger & 61 & 65 \\
\hline Autofahrer & 16 & 10 \\
\hline \hline \multirow{2}{*}{ Gesamt } & 335 & 396 \\
\cline { 2 - 3 } & \multicolumn{2}{|c|}{731} \\
\hline
\end{tabular}

Tabelle 13: Übersicht der auswertbaren Fragebögen

\subsection{Befragungsergebnisse der Radfahrer}

In den abgefragten Radfahrermerkmalen zeigten sich folgende Charakteristiken. Die befragten Radfahrer:

- $\quad$ sind zu 55 \% Männer und 45 \% Frauen,

- $\quad$ sind zu $90 \%$ zwischen 22 und 65 Jahren alt,

- besitzen zu $86 \%$ einen Führerschein,

- $\quad$ nutzen zu 98 \% das Rad mehrmals pro Woche,

- schätzen zu 82 \% ihre Fahrgeschwindigkeit ${ }^{3}$ zwischen 15 und 25 km/h ein,

- fahren zu $74 \%$ mehr als $31 \mathrm{~km}$ pro Woche und

- $\quad$ nutzen zu $80 \%$ den Streckenabschnitt mehrmals pro Woche.

\subsubsection{Flächennutzung der befragten Radfahrer}

$96 \%$ der befragten Radfahrer fuhren auf dem Radweg, $3 \%$ auf der Fahrbahn und $1 \%$ regelwidrig auf dem Gehweg. Unterschiede zwischen benutzungspflichtigen und nicht benutzungspflichtigen Radwegen waren nicht vorhanden. Die Ergebnisse bestätigten die Erkenntnisse der Erhebungen zur Flächennutzung in Kapitel 6.1. 97 \% der Radfahrer gaben an, immer diese Fläche zu nutzen.

Als Gründe für das Radwegfahren ergaben sich die 5 Kategorien von Motiven nach Abbildung 40. Die Sicherheit bzw. das Sicherheitsempfinden war hier zu $56 \%$ das wesentlichste Motiv der Radwegnutzung. Zu weiteren 36 \% waren es die Pflicht oder die Gewohnheit. Das Vorankommen und der Komfort waren nur untergeordnet. Bei Radwegen ohne Benutzungspflicht war es um $10 \%$ weniger die Pflicht, stattdessen um $10 \%$ mehr die Gewohnheit, welche die Radfahrer zum Radwegfahren veranlasste. Ansonsten waren die Motive der Radwegnutzung an benutzungspflichtigen und nicht benutzungspflichtigen Radwegen identisch.

Gründe für das Fahren auf der Fahrbahn waren fast ausschließlich das schnellere Vorankommen und der höhere Fahrkomfort. Unterschiede hinsichtlich der Radwegebenutzungspflicht existieren nicht. Insgesamt war das Fahrbahnfahren jedoch deutlich untergeordnet.

\footnotetext{
${ }^{3}$ Es handelt sich um die geschätzten Wunschgeschwindigkeiten bzw. Fahrgeschwindigkeiten auf unbehin-
} derter Strecke, dagegen nicht um Durchschnittsgeschwindigkeiten. 


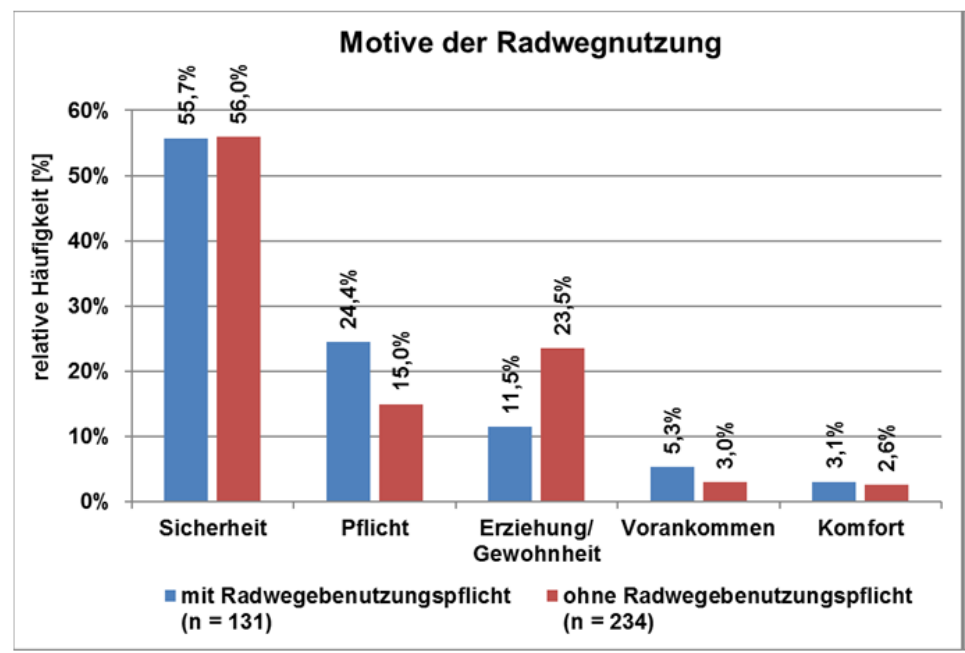

Abbildung 40: Gründe für die Radwegnutzung durch Radfahrer

$93 \%$ der befragten Radfahrer wechseln die genannte Fläche im Streckenverlauf nicht. Gründe für das Wechseln der Flächennutzung waren ausschließlich das schnellere Vorankommen. So erfolgte bei Stau auf der Fahrbahn ein Wechseln auf den Radweg bzw. aufgrund des Ausweichens vor Hindernissen auf dem Radweg (z.B. Fußgänger, Fahrzeuge) ein Wechseln auf die Fahrbahn.

Die Bedeutung der Sicherheit für die Wahl der Flächennutzung zeigt Abbildung 41. Die Sicherheit ist für $92 \%$ der befragten Radfahrer wichtig bei der Wahl der Flächennutzung. Für weniger als $0,5 \%$ der Radfahrer ist die Sicherheit nicht wichtig. Unterschiede hinsichtlich der Radwegebenutzungspflicht zeigten sich nicht. Bei der Bedeutung des Vorankommens waren die Ergebnisse deutlich ausgeglichener (vgl. Abbildung 41). Das schnelle Vorankommen ist an Radwegen mit Benutzungspflicht für $45 \%$ der Befragten wichtig, an Radwegen ohne Benutzungspflichtig nur für $34 \%$. Ursachen für diesen Unterschied zeigten sich nicht. Weiterhin ist das schnelle Vorankommen für jeden 5. Radfahrer bei der Wahl der Flächennutzung nicht entscheidend, dies unabhängig der Radwegebenutzungspflicht. Der Fahrkomfort wurde ähnlich dem Vorankommen bewertet.

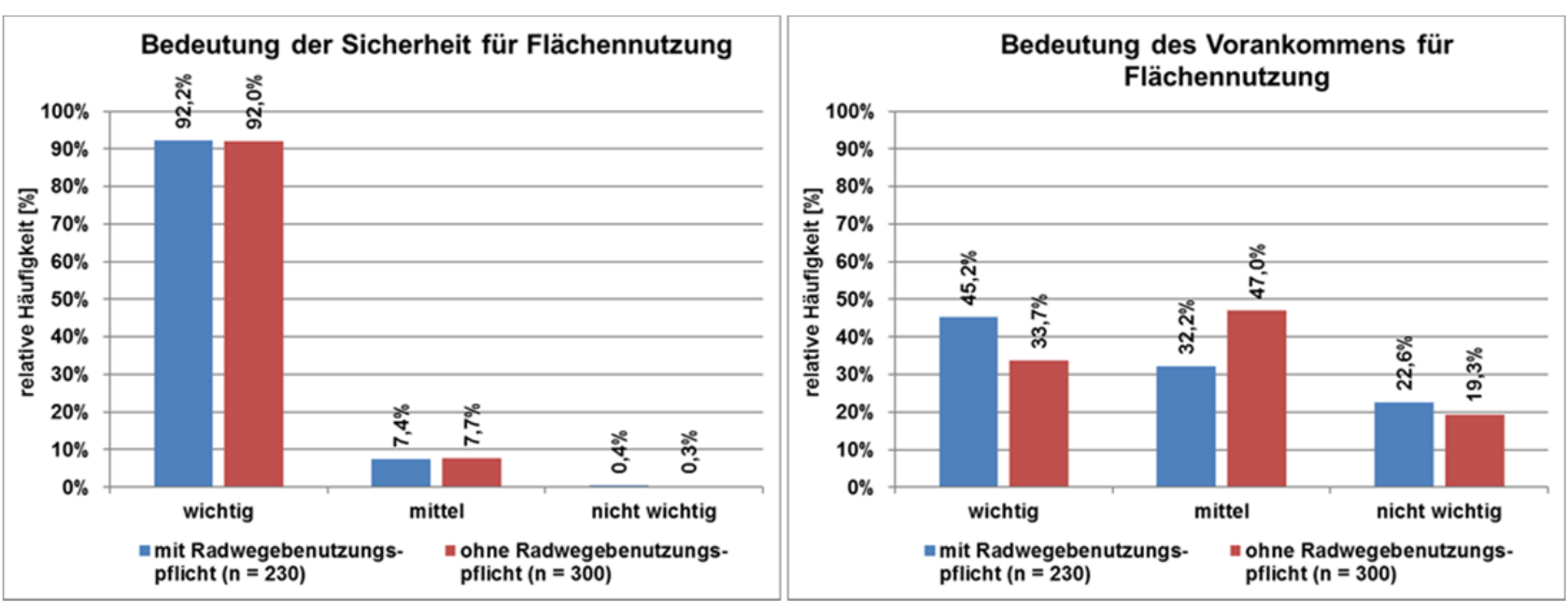

Abbildung 41: Bedeutung von Sicherheit und Vorankommen für die Wahl der Flächennutzung

Weiterhin wurden die Motive - Sicherheit, Vorankommen und Fahrkomfort - in der Rangfolge ihrer Bedeutung geordnet. Die Sicherheit war für $84 \%$ der Befragten auf Platz 1 dieser Rangfolge. Das schnelle Vorankommen war mit $11 \%$ untergeordnet, der Fahrkomfort unbedeutend. Unterschiede in der Radwegebenutzungspflicht waren nicht feststellbar. Somit besitzt die Sicherheit bei den Motiven der Flächennutzung insgesamt den höchsten Stellenwert. 
Die zu erwartende Verhaltensänderung mit Aufhebung der Radwegebenutzungspflicht war gering. Von den 213 befragten Nutzern der benutzungspflichtigen Radwege würden nach Aufhebung der Radwegebenutzungspflicht $89 \%$ weiterhin auf dem Radweg fahren. $10 \%$ der Radfahrer gaben an, später die Fahrbahn zu nutzen. Dies bestätigt die Ergebnisse zur Flächennutzung nach Kapitel 5.3.2 und Kapitel 6.1. Somit wird der Radweg auch ohne Benutzungspflicht in hohem Umfang weitergenutzt. Durch Differenzierung der Radfahrereigenschaften zeigte sich, dass tendenziell männliche, junge sowie viel und schnell fahrende Radfahrer nach Aufhebung der Radwegebenutzungspflicht das Fahrbahnfahren vorzögen.

Die Regelkenntnis der tatsächlich erlaubten Flächennutzung war schlecht. An Radwegen mit Benutzungspflicht dachten $43 \%$ der befragten Radfahrer, dass nur der Radweg genutzt werden darf. Bei nicht benutzungspflichtigen Radwegen waren dies $69 \%$. Dies bestätigt die Ergebnisse nach Alrutz et al. [2009] und GDV [2013]. Der geringe Anteil von Radfahrern, welche die verkehrsrechtliche Situation korrekt einschätzten (43\% an benutzungspflichtigen Radwegen, $26 \%$ an nicht benutzungspflichtigen Radwegen) verdeutlichte große Wissenslücken. Der Unterschied zwischen benutzungspflichtigen und nicht benutzungspflichtigen Radwegen ist den Radfahrern nicht bekannt.

\subsubsection{Bewertung der Interaktionen mit anderen Verkehrsteilnehmern}

Bei den wahrgenommenen Beeinträchtigungen zeigte sich, dass an benutzungspflichtigen Radwegen $54 \%$ und an nicht benutzungspflichtigen Radwegen $81 \%$ der befragten Radfahrer keine Beeinträchtigungen durch andere Verkehrsteilnehmer sahen (Abbildung 42). Aufgrund der überwiegenden Weiternutzung der Radwege konnte die Wahlfreiheit der Radfahrer nicht als Ursache bestätigt werden. Direkte Vergleiche waren hier jedoch aufgrund der überwiegenden MitOhne-Betrachtungen (vgl. Kapitel 6) nicht möglich.

Radfahrer fühlen sich vorrangig durch Pkw, darauffolgend durch Fußgänger und andere Radfahrer beeinträchtigt. Bei den Beeinträchtigungen durch Pkw wurde größtenteils von Problemen beim Abbiegen berichtet. Zudem werden Falschparker und hohe Verkehrsstärken als Beeinträchtigungen wahrgenommen. Bei Beeinträchtigungen durch Fußgänger wurde vorrangig Unachtsamkeit in Verbindung mit dem Betreten des Radweges genannt. Beeinträchtigungen durch andere Radfahrer werden durch Geschwindigkeitsunterschiede, Schwierigkeiten beim Überholen bzw. das Linksfahren hervorgerufen. Die von den Radfahrern empfundenen Beeinträchtigungen spiegeln sich auch in den Erläuterungen zu den beobachteten Konflikten nach Kapitel 6.2.2 wider.

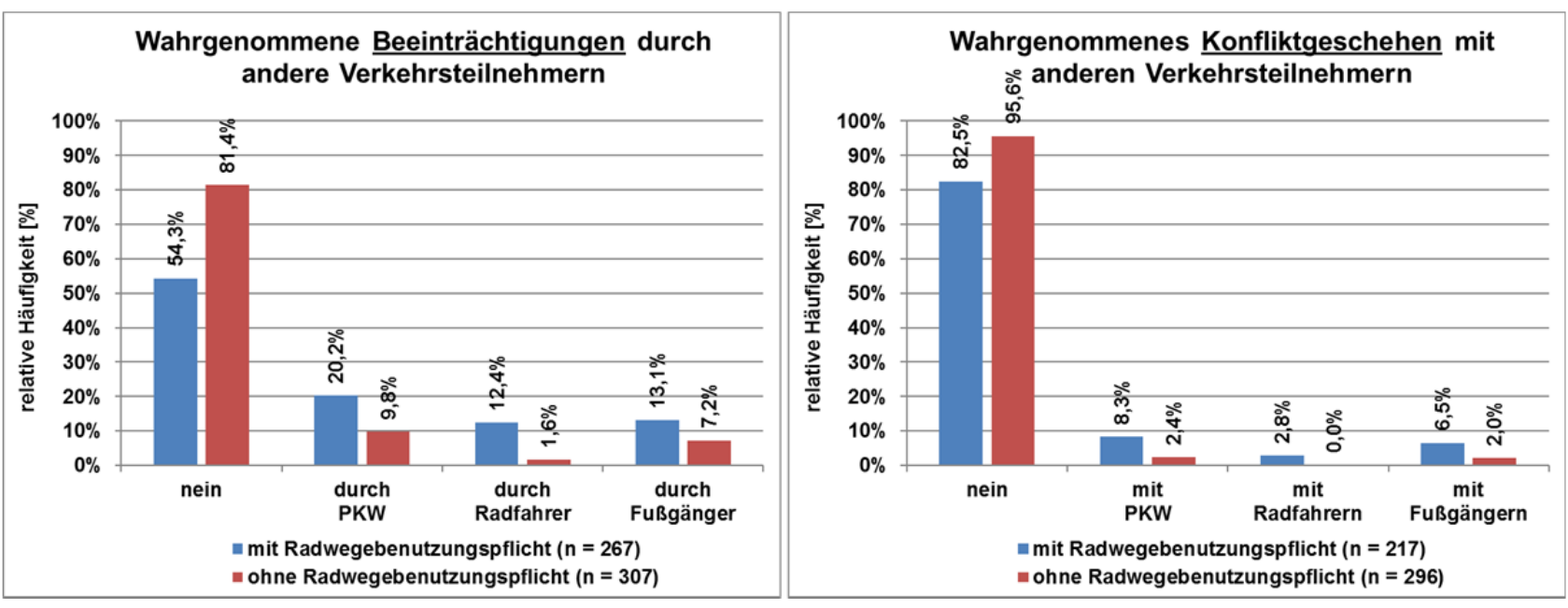

Abbildung 42: Wahrgenommene Beeinträchtigungen und Konflikte 
$17 \%$ der Radfahrer berichteten an benutzungspflichtigen Radwegen und $4 \%$ der Radfahrer an nicht benutzungspflichtigen Radwegen von erlebten Konflikten (vgl. Abbildung 42). Wie bei den Beeinträchtigungen handelte es sich bei den Konfliktgegnern dabei vorrangig um Pkw sowie um Fußgänger und andere Radfahrer. Da Beeinträchtigungen erlebt werden können, auch wenn dies nicht zu Konflikten führt, war die Anzahl der Konflikte hier geringer. Die Beschreibungen der Konflikte entsprachen den oben erläuterten Problembereichen der Beeinträchtigungen. Die erlebten Konflikte waren mit den beobachteten Konflikten der Verkehrsverhaltensanalyse vergleichbar.

\subsubsection{Bewertung der Verkehrssicherheit}

Die Sicherheitsbewertungen für das Fahren auf der knotenpunktfreien Strecke zeigt Abbildung 43, für Knotenpunkte Abbildung 44. Dabei wurde nach der tatsächlichen Flächennutzung differenziert.
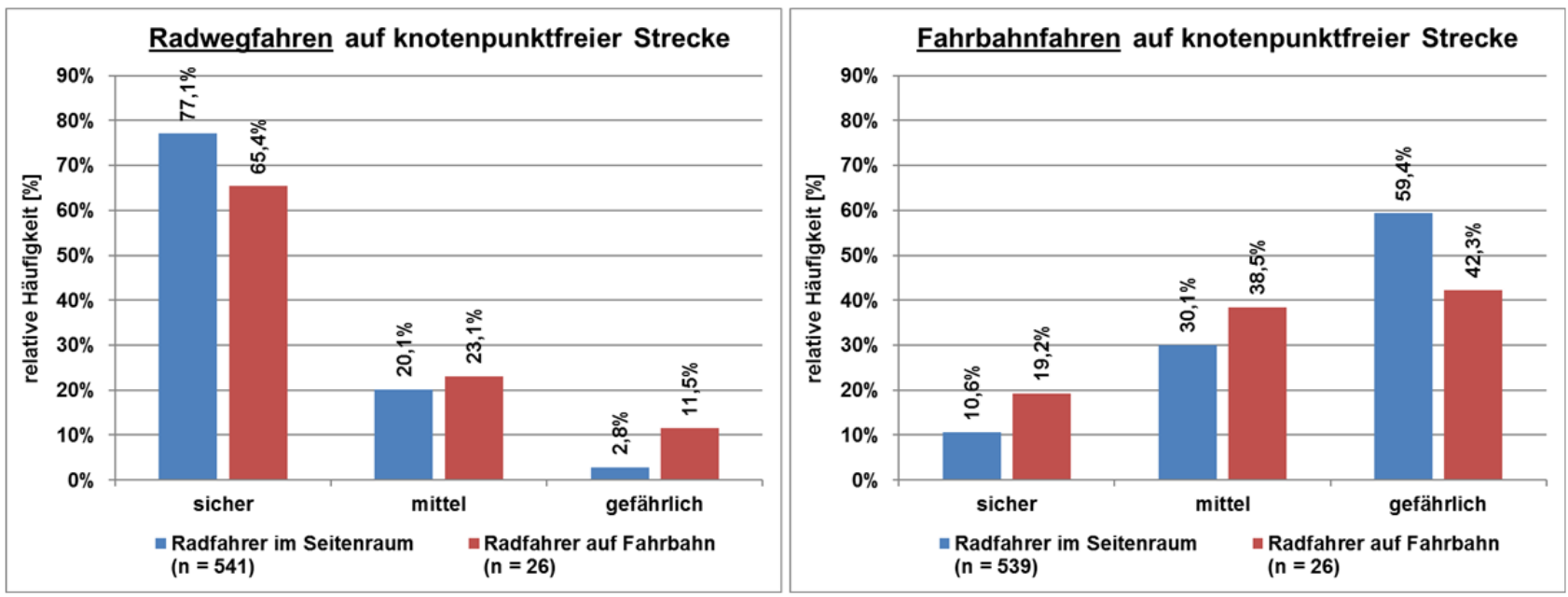

Abbildung 43: Sicherheitsbewertung für das Fahren auf der knotenpunktfreien Strecke
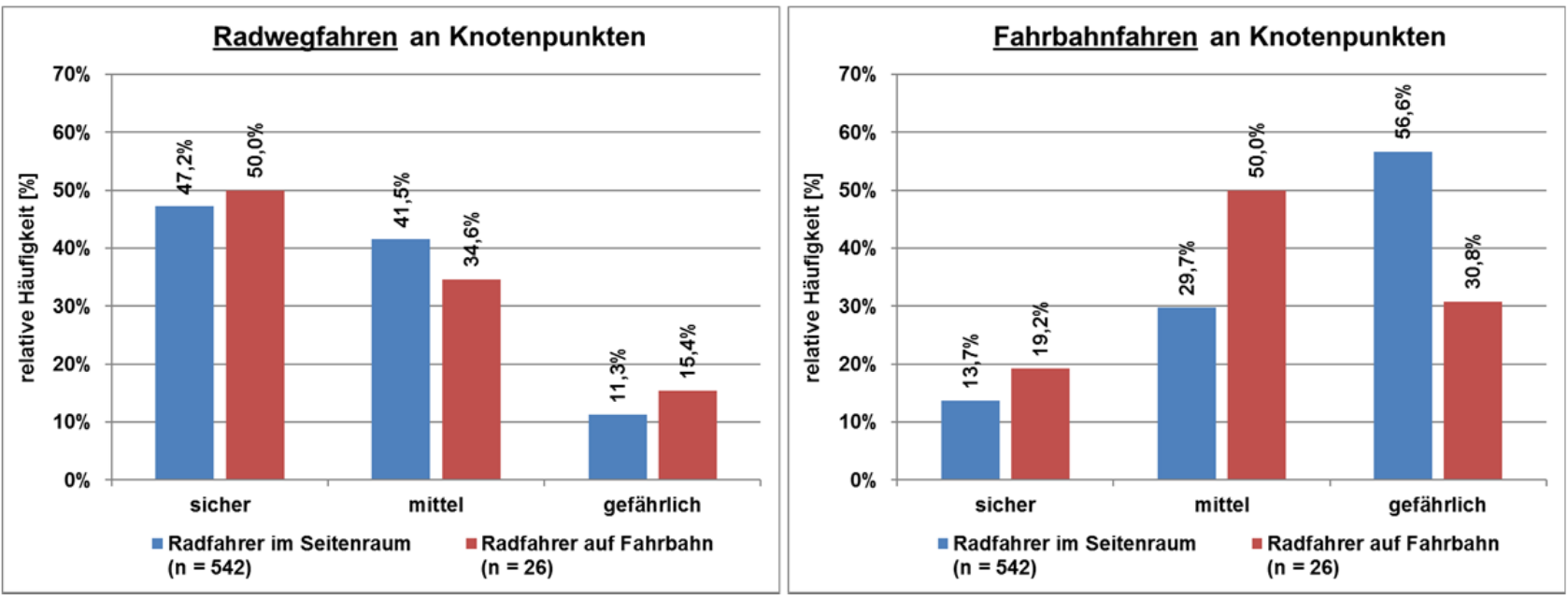

Abbildung 44: Sicherheitsbewertung für das Fahren an Knotenpunkten

$77 \%$ der befragten Radwegnutzer sahen das Radwegfahren auf der knotenpunktfreien Strecke als sicher an. Das Fahrbahnfahren wurde dagegen zu $60 \%$ als gefährlich bewertet. Von den Fahrbahnnutzern bewerteten das Fahren auf dem Radweg nur $65 \%$ als sicher, das Fahrbahnfahren dagegen $42 \%$ als gefährlich. An Knotenpunkten ergab sich mit deutlich ausgewogeneren Bewertungen ein vergleichbares Ergebnis beim Radwegfahren. Die Bewertungen des Fahrbahnfahrens an Knotenpunkten waren ähnlich denen der knotenpunktfreien Strecke. Diese Auswertung bestätigte das höhere subjektive Sicherheitsempfinden beim Fahren im Seitenraum. Nur maximal jeder 5. Radfahrer sah das Fahrbahnfahren als sicher an. Abschließend zeigten sich entgegengesetzte 
Bewertungen der Nutzergruppen. Das Radwegfahren wurde eher von Fahrbahnnutzern als gefährlich bewertet, das Fahrbahnfahren wiederum eher von Radwegnutzern.

\subsection{Befragungsergebnisse der Fußgänger und Autofahrer}

Nachfolgend werden die Ergebnisse der 126 befragten Fußgänger und 26 Autofahrer kurz zusammengefasst. Unabhängig der Radwegebenutzungspflicht nahmen etwa $19 \%$ der Fußgänger und $24 \%$ der Autofahrer Beeinträchtigungen durch Radfahrer war. Häufige Beeinträchtigungen aus Sicht der Fußgänger waren hohe Geschwindigkeiten, regelwidrige Gehwegnutzung, das Linksfahren und Unachtsamkeit der Radfahrer. Von Autofahrern wurden fehlende Rücksichtnahme sowie Probleme durch linksfahrende und schnelle Radfahrer als Beeinträchtigungen aufgeführt. Die wahrgenommenen Beeinträchtigungen ähneln sich über alle Verkehrsteilnehmergruppen hinweg.

$12 \%$ der Fußgänger und $32 \%$ der Autofahrer erlebten bereits Konflikte mit Radfahrern. Die Beschreibungen der Fußgänger glichen dabei denen der wahrgenommenen Beeinträchtigungen. Von Autofahrern erlebte Konflikte wurden größtenteils durch Abbiegevorgänge hervorgerufen. Weiterhin wurde die regelwidrige Fahrbahnnutzung von Radfahrern aus Sicht der Autofahrer genannt. Die Befragungen der Fußgänger und Autofahrer bestätigten insgesamt die aus Kapitel 7.1.2 bekannten Problembereiche aus der jeweils anderen Perspektive.

Hinsichtlich der Regelkenntnis bestätigten sich auch bei den Fußgängern und Autofahrern erhebliche Wissenslücken zur Radwegebenutzungspflicht.

Bei der Sicherheitsbewertung des Radfahrens auf dem betrachteten Streckenabschnitt ergaben sich über beide Verkehrsteilnehmergruppen hinweg zwei wesentliche Erkenntnisse. Zum einen wurde das Radwegfahren grundlegend als sicherer bewertet, zum anderen waren es die Knotenpunkte, denen die größte Gefährdung zugeschrieben wurde. Insgesamt bestätigte dies auch die Einschätzungen der Radfahrer.

\subsection{Fazit der Vor-Ort-Befragungen}

Bezüglich der Flächennutzung zeigten die Befragungen, dass die Radfahrer unabhängig der Benutzungspflicht zum Großteil auf dem Radweg fuhren. Mit Aufhebung der Radwegebenutzungspflicht sind zudem keine wesentlichen Verhaltensänderungen in der Flächennutzung zu erwarten. Dies bestätigte die bereits vorhandenen Ergebnisse aus Kapitel 5.3.2 und Kapitel 6.1. Der hohe Stellenwert der Verkehrssicherheit für die Wahl der Flächennutzung in Verbindung mit dem hohen Sicherheitsempfinden auf dem Radweg war hierfür die wesentliche Ursache. Auf der Fahrbahn wurde dagegen nur gefahren, um schneller voranzukommen.

In Hinblick auf die Regelkenntnis zeigten sich bei allen Verkehrsteilnehmergruppen erhebliche Wissenslücken in Bezug zur Radwegebenutzungspflicht. Dies bestätigte Untersuchungen von Alrutz et al. [2009] und GDV [2013]. Der Unterschied zwischen benutzungspflichtigen und nicht benutzungspflichtigen Radwegen ist den Verkehrsteilnehmern überwiegend nicht bekannt. In Verbindung mit dem tatsächlichen Verkehrsverhalten und dessen Motiven konnte geschlussfolgert werden, dass Verkehrsteilnehmer sich vielmehr an ihrem eigenen Sicherheitsempfinden orientierten, dagegen nicht an verkehrsrechtlichen Vorgaben hinsichtlich der Radwegebenutzungspflicht.

Bei den Auswertungen zu wahrgenommenen Beeinträchtigungen und erlebten Konflikten bestätigten sich grundlegend die Ergebnisse der Verkehrsverhaltensanalysen. Auch die Angaben der drei Verkehrsteilnehmergruppen waren nahezu identisch. Dies verdeutlichte, dass die Problemsituationen im Verkehrsverhalten unabhängig der jeweiligen Perspektive bekannt sind. 
Die Ergebnisse zur Sicherheitsbewertung des Radweg- und Fahrbahnfahrens waren ebenfalls über alle Verkehrsteilnehmergruppen vergleichbar. Hier bestätigte sich erneut das hohe subjektive Sicherheitsempfinden beim Radwegfahren. Weiterhin wurden auch die Knotenpunktbereiche einheitlich als Gefahrenschwerpunkte identifiziert. Somit war grundlegend ein Bewusstsein für kritische Verkehrssituationen vorhanden.

Abschließend spiegelten die hier erzielten Erkenntnisse die bisherigen Ergebnisse hinsichtlich der Flächennutzung (Kapitel 5.3.2 und Kapitel 6.1) sowie die Schwerpunkte der Unfallanalysen (vgl. Kapitel 5.3.3) und Verkehrsverhaltensbeobachtungen (vgl. Kapitel 6.2.2) wider. Ergänzend ist es mit den Vor-Ort-Befragungen gelungen, Erklärungen für die Verkehrsverhaltensweisen zu liefern. 


\section{Gesamtfazit der Untersuchung}

In diesem Kapitel werden die wesentlichen Kernaussagen aller einzelnen Arbeitsschritte kurz zusammenfasst (Kapitel 8.1), abschließend diskutiert (Kapitel 8.2) sowie Empfehlungen aus den vorliegenden Untersuchungen abgeleitet (Kapitel 8.3).

\subsection{Kurzzusammenfassung der Projektergebnisse}

In der Literatur existieren konkrete Bewertungen der Aufhebung der Radwegebenutzungspflicht nur nach Alrutz et al. [2009]. Hier hat sich an 10 Radwegen mit Aufhebung der Radwegebenutzungspflicht (ohne Begleitmaßnahmen) keine Verschlechterung der Verkehrssicherheit eingestellt. Mögliche Veränderungen sind aber auch abhängig von Änderungen der Flächennutzung (tatsächliche Fahrbahnnutzung), welche bisher kaum feststellbar waren (vgl. Alrutz et al. [2009] und GDV [2013]). Vermutet wird hier ein höheres subjektives Sicherheitsempfinden beim Radwegfahren.

Die subjektiven Einschätzungen der kommunalen Entscheidungsträger aus der Online-Umfrage zeigen keine einheitlichen Vorgehensweisen beim Umgang mit der Radwegebenutzungspflicht in Deutschland. Dies verdeutlichen die verschiedenartigen umgesetzten Begleitmaßnahmen und die Vielzahl von Anordnungskriterien der Radwegebenutzungspflicht. Bezüglich der Effekte der Aufhebung der Benutzungspflicht existieren in den Kommunen mitunter sehr unterschiedliche Meinungen. Vergleichsweise einheitlich wird dagegen von Akzeptanzproblemen bei Radfahrern und Autofahrern ausgegangen, wenn Radfahrer die Fahrbahn nutzen.

Die Unfallanalyse zeigt eine Zunahme der Radunfälle an den untersuchten Radwegen mit Aufhebung der Radwegebenutzungspflicht (ohne Begleitmaßnahmen). Dieses konnte aber auf eine Zunahme des Radverkehrsaufkommens zurückgeführt werden. Mit Berücksichtigung der steigenden Radverkehrsentwicklung bei der Unfallkenngrößenberechnung war keine Veränderung der Verkehrssicherheit feststellbar. Schwerpunkte im Unfallgeschehen sind Knotenpunkte. In Kurzzeitzählungen zur Ermittlung der Expositionsgrößen der Unfallkenngrößenberechnung bestätigt sich eine nur geringe Fahrbahnnutzung nach Aufhebung der Benutzungspflicht. Entsprechend zeigen auch die Unfallmerkmale kaum strukturelle Veränderungen bzw. Verlagerungen auf die Fahrbahn.

Die Verkehrsverhaltensanalyse verdeutlicht kaum Fahrbahnnutzung bei keinen oder nur geringfügigen Begleitmaßnahmen (Piktogrammen) mit Aufhebung der Radwegebenutzungspflicht. Weiterhin zeigt sich hier ein Rückgang des Fahrbahnfahrens bei hohen Kraftfahrzeugverkehrsstärken, schmalen Fahrstreifen und breiten nicht benutzungspflichtigen Radwegen. Deutliche Verlagerungen auf die Fahrbahn ergeben sich nur bei Schutz-/Radfahrstreifen und Rückbau des Radweges. Die Schwerpunkte im Konfliktgeschehen bestätigen die Unfallanalyse. Zudem vermeidet die Aufhebung der Radwegebenutzungspflicht keine Konflikte, da keine Verhaltensänderung erfolgt.

Die Vor-Ort-Befragungen der Verkehrsteilnehmer verdeutlichen für nicht benutzungspflichtige Radwege ohne Begleitmaßnahmen, warum keine wesentlichen Verhaltensänderungen eintreten. Der hohe Stellenwert der Verkehrssicherheit für die Wahl der Flächennutzung der Radfahrer und das hohe subjektive Sicherheitsempfinden beim Radwegfahren zeigen sich als Motive für die Weiternutzung der Radwege. Weiterhin sind den Radfahrern die verkehrsrechtlichen Regelungen zur Radwegebenutzungspflicht größtenteils unbekannt. Radfahrer handeln stattdessen aufgrund ihrer individuellen Sicherheitsempfindungen. Die Konflikt- bzw. Gefahrenschwerpunkte im städtischen Radverkehr sind den Verkehrsteilnehmern dabei überwiegend bekannt.

Abschließend hat die vorliegende Untersuchung gezeigt, dass aufgrund fehlender Verhaltensänderungen mit Aufhebung der Radwegebenutzungspflicht auch keine wesentlichen Änderungen im Unfall- und Konfliktgeschehen eintreten. 


\subsection{Diskussion der Projektergebnisse}

Ziel des vorliegenden Projektes war die Bewertung der Veränderungen von Verkehrssicherheit und Verkehrsverhalten mit Aufhebung der Radwegebenutzungspflicht mit einem umfangreichen Streckenkollektiv sowie unter Berücksichtigung unterschiedlicher Begleitmaßnahmen.

Die Untersuchung zeigt, dass die Erhöhung der Unfallzahlen in der Gesamtentwicklung vor und nach Aufhebung der Radwegebenutzungspflicht (ohne Begleitmaßnahmen) im Wesentlichen mit der Radverkehrsentwicklung der vergangenen Jahre erklärt werden kann (siehe Kapitel 5.3.1). Verbesserungen oder Verschlechterungen der Verkehrssicherheit mit Aufhebung der Radwegebenutzungspflicht (ohne Begleitmaßnahmen) können hier nicht nachgewiesen werden. Dies bestätigt die Ergebnisse bisheriger Untersuchungen von Alrutz et al. [2009].

Zudem zeigt die gemeinsame makroskopische und mikroskopische Unfallanalyse (Kapitel 5.3.3), dass sich die Steigerung im Radverkehrsaufkommen geringfügig mehr in bestimmten Unfallmerkmalen niederschlägt, in anderen hingegen weniger. Diese unterschiedlichen Ergebnisse verweisen zwar auf gewisse Schwerpunkte im Unfallgeschehen, die sich in der Gesamtentwicklung gegenseitig jedoch wieder aufwiegen. Die Aufhebung der Radwegebenutzungspflicht ist hierfür nicht ursächlich. Wesentliche strukturelle Änderungen im Unfallgeschehen konnten mit der Untersuchung nicht bestätigt werden. Entsprechend zeigen sich auch keine klaren positiven oder negativen Effekte hinsichtlich der Sicherheit des städtischen Radverkehrs.

Ursache für die ausgebliebenen strukturellen Änderungen im Unfallgeschehen sind die ausgebliebenen Veränderungen im Verkehrsverhalten der Radfahrer. An nicht benutzungspflichtigen Radwegen ohne weitere Begleitmaßnahmen zeigen sich kaum Verlagerungseffekte der Radfahrer auf die Fahrbahn (durchschnittlich fahren nur etwa $10 \%$ auf der Fahrbahn). Dies zeigen die Ergebnisse nach Kapitel 5.3.2 sowie Kapitel 6.1, welche insgesamt die Ergebnisse von Alrutz et al. [2009] bestätigen.

Aufgrund der ausbleibenden Veränderungen im Verkehrsverhalten bzw. der vorrangigen Weiternutzung der nicht benutzungspflichtigen Radwege (ohne weitere Begleitmaßnahmen) stellen sich mit Aufhebung der Radwegebenutzungspflicht sowohl im Unfallgeschehen als auch im Konfliktgeschehen keine Änderungen ein. Neue Konflikte auf der Fahrbahn zeigten sich nicht.

Als Motive der Radwegweiternutzung zeigt die Vor-Ort-Befragung in Kapitel 7.1 ein höheres subjektives Sicherheitsempfinden. Radwege sind somit die präferierte Führungsform der Radfahrer. Dies bestätigt die Ergebnisse von GDV [2013]. Das Unsicherheitsempfinden beim Fahrbahnfahren ist ebenfalls stark ausgeprägt. Hier wird nur aufgrund des schnelleren Vorankommens gefahren.

Die Ergebnisse der infrastrukturellen Analyse an Radwegen ohne Begleitmaßnahmen (Kapitel 5.3.1) zeigen tendenziell ein höheres Unfallrisiko bei:

- geringen Kraftfahrzeugverkehrsstärken (Belastungsbereich II),

- Radverkehrsstärken von mehr als 250 Radfahrern pro Tag und Richtung,

- $\quad$ mehr als 2 Fahrstreifen pro Richtung und

- besonders schmalen und breiten Fahrsteifen.

Die Untersuchung zeigt hier nur Indizien für bestimmte Entwicklungen. Empfehlungen für die Anordnung einer benutzungspflichtigen Radverkehrsanlage aus Sicherheitsgründen können im Rahmen des Projektes nicht abgeleitet werden. Für Anordnungskriterien einer Radwegebenutzungspflicht aus Gründen der Verkehrssicherheit bzw. einer besonderen Gefahrenlage besteht daher weiterer Forschungsbedarf. Auch die Umsetzung von bestimmten Begleitmaßnahmen mit Aufhe- 
bung der Radwegebenutzungspflicht bleibt in Abhängigkeit der individuellen Streckeneigenschaften eine Einzelfallentscheidung. Pauschale Empfehlungen sind hierfür nicht möglich. Das vorliegende Projekt kann allerdings Hinweise auf die möglichen Effekte bezüglich der Verkehrssicherheit und Flächennutzung für bestimmte Begleitmaßnahmen geben.

Neben den vorrangig untersuchten Radwegen ohne weitere Begleitmaßnahmen mit Aufhebung der Radwegebenutzungspflicht (insgesamt drei Viertel der betrachteten Radwege) sind Aussagen zu weiteren Begleitmaßnahmen (Markierung von Schutzstreifen, Piktogrammen und Anordnung Gehweg Radfahrer frei) aufgrund der geringen Stichprobenumfänge dabei nur eingeschränkt möglich. Die Erhöhungen der Unfallraten auf der knotenpunktfreien Strecke bei Markierung von Schutzstreifen und Radfahrerpiktogrammen können nur auf Ausreißer zurückgeführt werden. Ohne deren Effekte sind auch hier keine Veränderung der Verkehrssicherheit erkennbar. An Knotenpunkten zeigen sich dagegen Verbesserungen der Verkehrssicherheit, wenn Schutzstreifen markiert und Radwege zurückgebaut werden (siehe Kapitel 5.3.1). Zur Verkehrssicherheit von Schutzund Radfahrstreifen sowie auch die verbleibenden Begleitmaßnahmen besteht jedoch weiterer Forschungsbedarf.

Hinsichtlich der Verlagerungseffekte auf die Fahrbahn bei anderen Begleitmaßnahmen zeigt die Untersuchung folgendes Ergebnis. Deutliche Verlagerungen haben sich hier nur bei der Markierung von Schutzstreifen inkl. Radwegrückbau (84 \% nach Kapitel 5.3.2) und Radfahrstreifen inkl. Radwegrückbau (65 \% nach Kapitel 6.1) gezeigt, da Angebote auf der Fahrbahn geschaffen werden. Für Piktogramme konnte dagegen keine Wirkung festgestellt werden.

Im Rahmen der Untersuchung konnte weiterhin das besondere Risiko der potentiellen Doppelführung des Radverkehrs an nicht benutzungspflichtigen Radwegen aufgrund ausgebliebener Verhaltensänderungen nicht bestätigt werden. Dies widerspricht den Erwartungen aus der Literatur (Kapitel 2.5) und den subjektiven Erwartungen der kommunalen Entscheidungsträger (Kapitel 3.1.2).

Die Vor-Ort-Befragungen nach Kapitel 7.1 zeigen, dass individuelle Sicherheitsempfindungen maßgebend für das Verkehrsverhalten sind, jedoch nicht die verkehrsrechtlichen Randbedingungen bezüglich der Radwegebenutzungspflicht. Entsprechend ändert sich auch allein durch die Änderung der Radwegebenutzungspflicht die Verkehrssicherheit nicht (siehe Kapitel 5.3.1). Dies zeigten auch Alrutz et al. [2009]. Hiernach ist die Benutzungspflicht für die Sicherheit von Radwegen nicht ausschlaggebend, dagegen wird sicherheitsrelevanten Anlagenmängeln und Entwurfsdefiziten ein vorrangiger Stellenwert zugesprochen.

Weiterhin zeigt sich deutlich, dass bei allen Verkehrsteilnehmergruppen (Radfahrer, Fußgänger, Autofahrer) die Problemsituationen im städtischen Verkehr sowie die Gefahrenschwerpunkte bekannt sind. Somit existiert ein bestimmtes Bewusstsein für kritische Situationen im Verkehr. Die hier berichteten Konflikte bestätigen auch die Erwartungen der Literaturanalyse (Kapitel 2.5.1) und der kommunalen Entscheidungsträger (Kapitel 3.1.2), generell aber auch die Schwerpunkte der Unfallanalyse (Kapitel 5.3.3) und die beobachteten Konflikte nach Kapitel 6.2.

Die Ergebnisse der Vor-Ort-Befragungen zeigen abschließend, dass ein Großteil der Befragten den Unterschied zwischen benutzungspflichtigen und nicht benutzungspflichtigen Radwegen offensichtlich gar nicht kennt (vgl. Kapitel 7.1.1). Auch nach Alrutz et al. [2009] und GDV [2013] ist das Verkehrsverhalten im Radverkehr durch Regelunkenntnis geprägt. Hinsichtlich der Wissenslücken zu regelkonformen Verhalten besteht somit weiterer Aufklärungsbedarf. 


\subsection{Empfehlungen zur Radwegebenutzungspflicht}

Für Entscheidungen zur Aufhebung der Radwegebenutzungspflicht sowie zur Verkehrssicherheit nicht benutzungspflichtiger Radwege sind basierend auf den Projektergebnissen die folgenden Empfehlungen möglich. Die Aussagen beziehen sich dabei vorrangig auf nicht benutzungspflichtige Radwege ohne weitere Begleitmaßnahmen, welche hiermit am umfassendsten untersucht sind.

Die vorliegenden Ergebnisse zeigen, dass Radwege mit Benutzungspflicht per se nicht sicherer oder unsicherer sind als Radwege ohne Benutzungspflicht. Entsprechend zeigt auch die Aufhebung der Radwegebenutzungspflicht in der Regel keine Auswirkungen auf die Verkehrssicherheit, da ohne weitere Begleitmaßnahmen keine wesentlichen Änderungen in der Flächennutzung und damit auch nicht im Unfall- und Konfliktgeschehen feststellbar sind. Die Benutzungspflicht einer Radverkehrsanlage bzw. die Aufhebung der Benutzungspflicht ist somit nicht wesentlich für die Verkehrssicherheit.

Unter bestimmten Randbedingungen kann die Radwegebenutzungspflicht dennoch sinnvoll sein. Die Definition konkreter einheitlicher Anordnungskriterien war jedoch auch im Rahmen des vorliegenden Projektes nicht möglich. Wesentliche Ursache hierfür ist, dass Veränderungen im Verkehrsverhalten bzw. in der Flächennutzung auf den Untersuchungsstrecken im Projekt größtenteils ausgeblieben sind. Zudem deutet sich an, dass das bisher in der StVO enthaltene Anordnungskriterium der Gefahrenlage aufgrund der Vielzahl sicherheitsrelevanter Einflussfaktoren nur schwer pauschal zu konkretisieren ist. Die Entscheidung über die Benutzungspflicht einer Radverkehrsanlage wird daher immer eine Einzelfallentscheidung bleiben.

Seitens städtischer Entscheidungsträger ist bei Entscheidungen zur Radwegebenutzungspflicht zu beachten, dass

- nicht benutzungspflichtige Radwege überwiegend weitergenutzt werden,

- $\quad$ somit nur geringfügige Verlagerungen im Unfallgeschehen und

- ohne konkrete bauliche Maßnahmen in der Regel keine Veränderungen der Verkehrssicherheit zu erwarten sind.

Es muss also beachtet werden, dass allein die Aufhebung der Radwegebenutzungspflicht nicht ausreichend ist, um der Radverkehrsentwicklung und den Sicherheitsbedürfnissen des Radverkehrs zu entsprechen oder die Verkehrssicherheit des Radverkehrs zu erhöhen. Daher muss grundlegend neben der Aufhebung der Radwegebenutzungspflicht ein besonderer Fokus der Verkehrssicherheitsarbeit auf die Beseitigung bereits bekannter und hier erneut nachgewiesener Unfall- und Konfliktschwerpunkte gelegt werden.

Das Nutzen oder Nichtnutzen der Fahrbahn an nicht benutzungspflichtigen Radwegen bleibt abschließend eine Entscheidung des Radfahrers. Das vorliegende Projekt zeigt, dass bei bestimmten Randbedingungen die Meidung des Fahrbahnfahrens sehr deutlich ist (z.B. bei hohen Kraftfahrzeugverkehrsstärken oder gut ausgebauten Radwegen). Somit wäre hier die Bereitstellung ausreichend dimensionierter und sicherer Radverkehrsanlagen in jedem Fall erforderlich, um den Radverkehr nicht aufgrund schlechter Radwege bei ungünstigen Randbedingungen auf die Fahrbahn zu zwingen.

Die Bereitstellung guter Radwege führt auch zu einer hohen Nutzungsakzeptanz. Entsprechen Radwege nicht diesen Standards, dient die Aufhebung der Radwegebenutzungspflicht nicht dem Freikauf von der Verantwortung, geeignete Radverkehrsanlagen herzustellen. Somit müssen vorhandene Radwege unabhängig von deren Benutzungspflicht einen ausreichenden Standard auf- 
weisen. Hierbei ist den Knotenpunktbereichen besondere Aufmerksamkeit zu schenken. Zusammengefasst sind sichere infrastrukturelle Angebote zu schaffen, die den Anforderungen des Radverkehrs genügen und dessen Entwicklung entsprechen. 


\section{Literaturverzeichnis}

\section{Artikel, Berichte und Regelwerke:}

\section{[Ahrens et al. 2010]}

Ahrens, G.-A./Ließke, F./Wittwer, R./Hubrich, S.: Sonderauswertung zur Verkehrserhebung „Mobilität in Städten - SrV 2008“ - Städtevergleich, Dresden, 2010

\section{[Ahrens et al. 2015]}

Ahrens, G.-A./Wittwer, R./Hubrich, S./Wittig, S./Ließke, F.: Sonderauswertung zum Forschungsprojekt „Mobilität in Städten - SrV 2013“ - Städtevergleich, Dresden, 2015

\section{[Alrutz 2015]}

Alrutz, D.: Handlungsempfehlungen zur Radwegebenutzungspflicht, Webinar der Fahrradakademie des Deutschen Instituts für Urbanistik gGmbH, 17.11.2015

\section{[Alrutz et al. 1998]}

Alrutz, D./Hülsen, H./Ruwenstroth, G.: Neuregelung der Radwegebenutzungspflicht nach der StVO und VwV-StVO, in: Straßenverkehrstechnik, Heft 3, Seiten 117-122, 1998

\section{[Alrutz et al. 2009]}

Alrutz, D./Bohle, W./Müller, H./Prahlow, H./Hacke, U./Lohmann, G.: Unfallrisiko und Regelakzeptanz von Fahrradfahrern, Berichte der Bundesanstalt für Straßenwesen, Heft V 184, Bergisch Gladbach, 2009

\section{[Alrutz et al. 2015]}

Alrutz, D./Bohle, W./Busek, S.: Nutzung von Radwegen in Gegenrichtung - Sicherheitsverbesserungen, Berichte der Bundesanstalt für Straßenwesen, Heft V 261, Bergisch Gladbach, 2015

\section{[Arnold et al. 2008]}

Arnold, M./Hedeler, M./Wöppel, H.-D./Dahme, J.: Hochrechnungsverfahren für Kurzzeitzählungen auf Hauptverkehrsstraßen in Großstädten, Forschung Straßenbau und Straßenverkehrstechnik, Heft 1007, Bonn, 2008

\section{[Baier et al. 2013]}

Baier, R./Göbbels, A./Klemps-Kohnen, A.: Sicherheitskenngrößen für den Radverkehr, Berichte der Bundesanstalt für Straßenwesen, Heft V 228, Bergisch Gladbach, 2013

\section{[BBSR 2014]}

Bundesinstitut für Bau-, Stadt- und Raumforschung (BBSR): Laufende Stadtbeobachtung - Raumabgrenzungen - Stadt- und Gemeindetypen in Deutschland, Online unter URL:

http://www.bbsr.bund.de/BBSR/DE/Raumbeobachtung/Raumabgrenzungen/StadtGemeindetyp/St adtGemeindetyp node.html [Stand: 17.01.2017]

\section{[Below 2016]}

Below, A. von: Verkehrssicherheit von Radfahrern - Analyse sicherheitsrelevanter Motive, Einstellungen und Verhaltensweisen, Berichte der Bundesanstalt für Straßenwesen, Heft M 264, Bergisch Gladbach, 2016

\section{[BMVBS 2010]}

Bundesministerium für Verkehr, Bau und Stadtentwicklung (BMVBS): Pressemitteilung (103/2010) vom 13.04.2010, Online unter URL:

http://www.bmvi.de/SharedDocs/DE/Pressemitteilungen/2010/103-ramsauer-alte-verkehrsschilderbleiben-gueltig.html [Stand: 05.11.2015] 


\section{[BMVBS 2012]}

Bundesministerium für Verkehr, Bau und Stadtentwicklung (BMVBS): Verordnung zur Neufassung der Straßenverkehrs-Ordnung (StVO) vom 26.07.2012, Berlin, 2012

\section{[Böhmer 2015]}

Böhmer, T.: Hinweise des Projektleiters zur Verwendung der Daten des ADFC-FahrradklimaTests, Online unter URL: http://www.adfc.de/fahrradklima-test/ergebnisse/adfc-fahrradklima-test2014---die-ergebnisse [Stand: 23.09.2015]

\section{[DESTATIS 2015]}

Statistisches Bundesamt (DESTATIS): Gemeindeverzeichnis - Gebietsstand: 31.03.2015 (1. Quartal), Wiesbaden, 2015

\section{[Draeger 1997]}

Draeger, W.: Die StVO-Novelle - Konsequenzen für die Radverkehrsplanung, in: Straßenverkehrstechnik, Heft 12, Seiten 581-596, 1997

\section{[EFA 2002]}

Forschungsgesellschaft für Straßen- und Verkehrswesen (FGSV): Empfehlungen für Fußgängerverkehrsanlagen (EFA), Köln, 2002

\section{[EPOMM 2015]}

European Platform on Mobility Management (EPOMM): TEMS - The EPOMM Modal Split Tool, Online unter URL: http://www.epomm.eu/tems/index.phtml [Stand: 23.09.2015]

\section{[ERA 2010]}

Forschungsgesellschaft für Straßen- und Verkehrswesen (FGSV): Empfehlungen für Radverkehrsanlagen (ERA), Köln, 2010

\section{[Erke et al. 1985]}

Verkehrskonflikttechnik: Handbuch für die Durchführung und Auswertung von Erhebungen, Unfallund Sicherheitsforschung Straßenverkehr, Heft 52, Bremerhaven, 1985

\section{[FGSV 1998]}

Forschungsgesellschaft für Straßen- und Verkehrswesen (FGSV): Hinweise zur Beschilderung von Radverkehrsanlagen nach der Allgemeinen Verwaltungsvorschrift zur Straßenverkehrs-Ordnung, Köln, 1998

\section{[FGSV 2003]}

Forschungsgesellschaft für Straßen- und Verkehrswesen (FGSV): Merkblatt für die Auswertung von Straßenverkehrsunfällen, Teil 1: Führen und Auswerten von Unfalltypen-Steckkarten, Köln, 2003

\section{[FGSV 2016]}

Mitarbeiter des Arbeitskreises 3.3.6 „Aktuelle Themen der Lichtsignalsteuerung“ der Forschungsgesellschaft für Straßen- und Verkehrswesen (FGSV): Auswirkungen der Aufhebung der Radwegebenutzungspflicht an lichtsignalisierten Knotenpunkten, in: Straßenverkehrstechnik, Heft 5, Seiten 281-285, 2016

\section{[GDV 2013]}

Kolrep-Rometsch, H./Leitner, R./Platho, C./Richter, T./Schreiber, A./Schreiber, M./Butterwegge, P.: Abbiegeunfälle Pkw/Lkw und Fahrrad, Forschungsbericht Nr. 21 der Unfallforschung der Versicherer im Gesamtverband der deutschen Versicherungswirtschaft, Berlin, 2013 


\section{[GDV 2015]}

Alrutz, D./Bohle, W./Maier, R./Enke, M./Pohle, M./Zimmermann, F./Ortlepp, J./Schreiber, M.: Einfluss von Radverkehrsaufkommen und Radverkehrsinfrastruktur auf das Unfallgeschehen, Forschungsbericht Nr. 29 der Unfallforschung der Versicherer im Gesamtverband der deutschen Versicherungswirtschaft, Berlin, 2015

\section{[HBS 2001]}

Forschungsgesellschaft für Straßen- und Verkehrswesen (FGSV): Handbuch für die Bemessung von Straßenverkehrsanlagen (HBS), Köln, 2001

\section{[Horn 2015]}

Horn, B.: Geschichte der städtischen Radverkehrsplanung, in: Bracher et al. (Hrsg.): Handbuch der kommunalen Verkehrsplanung, Kapitel 2.1.1.2, 32. Ergänzungslieferung 08/2002, Berlin, 2015

\section{[Klöpfer et al. 2011]}

Klöpfer, J./Mayer-Zawar, B.: Leitfaden zur Überprüfung der Radwegebenutzungspflicht in Mainz, Mainz, 2011

\section{[Landeshauptstadt München 2016]}

Vorher-Nachher-Untersuchung - Duale Führung mit Schutzstreifen und Radwegen ohne Benutzungspflicht in der Elsenheimerstraße, Kurzbericht, München, 2016

\section{[Netter 2014]}

Netter, A.: Aktuelle Rechtsfragen der Ortsdurchfahrten, in: Durner, W. (Hrsg.): Die Stellung der Gemeinden im Straßenrecht - aktuelle Probleme des Umweltrechts, Berichte der Bundesanstalt für Straßenwesen, Heft S 83, Bergisch Gladbach, 2014

\section{[NLStbV 2013]}

Niedersächsische Landesbehörde für Straßenbau und Verkehr (NLStbV): Leitfaden Radverkehr Radverkehrsführung - Radwegebenutzungspflicht - Kostenträgerschaft - Baulast für Radwege an Bundes- und Landesstraßen in Niedersachsen, Hannover, 2013

\section{[Ohm et al. 2015]}

Ohm, D./Fiedler, F./Zimmermann, F./Kraxenberger, T./Maier, R./Hantschel, S./Otto, M.: Führung des Radverkehrs im Mischverkehr auf innerörtlichen Hauptverkehrsstraßen, Berichte der Bundesanstalt für Straßenwesen, Heft V 257, Bergisch Gladbach, 2015

\section{[RASt 2006]}

Forschungsgesellschaft für Straßen- und Verkehrswesen (FGSV): Richtlinien für die Anlage von Stadtstraßen (RASt), Köln, 2006

\section{[RiLSA 2010]}

Forschungsgesellschaft für Straßen- und Verkehrswesen (FGSV): Richtlinien für Lichtsignalanlagen (RiLSA), Köln, 2010

\section{[Schiller 2011]}

Schiller, C./Zimmermann, F./Bohle, W.: Hochrechnungsmodell von Stichprobenzählungen für den Radverkehr - Leitfaden zur Anwendung des Excel-Tools und des vereinfachten Verfahrens, unveröffentlichter Bericht zum Forschungsprojekt FE 77.495/2008 im Auftrag des Bundesministeriums für Verkehr, Bau- und Stadtentwicklung (BMVBS), Dresden, 2011 


\section{[Schmidt 1996]}

Schmidt, G./Thomas, B.: Hochrechnungsfaktoren für manuelle und automatische Kurzzeitzählungen im Innerortsbereich, Forschung Straßenbau und Straßenverkehrstechnik, Heft 732, Bonn-Bad Godesberg, 1996

\section{[SHP 2013]}

SHP Ingenieure (SHP): Gefährdungspotential für Radfahrer und Benutzungspflicht für Radverkehrsanlagen - Region Hannover, unveröffentlichter Endbericht im Auftrag der Region Hannover, Hannover, 2013

\section{[Stadt Münster 2007]}

Stadt Münster: Signale für den Radverkehr - Ein Leitfaden zur Radverkehrssignalisierung, Hannover, 2007

\section{[Stephan et al. 2010]}

Stephan, R. R./Alrutz, D./Kalle, U./Majcherek, J./Mischer, W.: 46. Änderung der StVO - Schwerpunkt Radverkehr, in: Straßenverkehrstechnik, Heft 1, Seiten 26-31, 2010

\section{Gesetzestexte und Verordnungen:}

\section{[DL 1992]}

Nuovo codice della Strada, DL 30.04.1992, n²85, 1992, Online unter URL:

http://www.mit.gov.it/mit/site.php?p=normativa\&o=vd\&id=1\&id cat=\&id dett=0 (Übersetzung durch Filippo Moraldi, Università degli Studi di Firenze) [Stand: 10.11.2015]

\section{[DPR 1992]}

Regolamento di esecuzione e di attuazione del nuovo codice della strada, DPR 16.12.1992, $n^{\circ} 495$, 1992, Online unter URL: http://www.normattiva.it/uri-

res/N2Ls?urn:nir:stato:decreto.del.presidente.della.repubblica:1992-12-16;495 (Übersetzung durch Filippo Moraldi, Università degli Studi di Firenze) [Stand: 10.11.2015]

\section{[IISR 2011]}

Instruction Interministérielle sur la signalisation routière du 22 octobre 1963 (IISR) - 7ème PARTIE: Marques sur chaussée, modifié par les arrêtés du 6 décembre 2011 (J.O. du 22 décembre 2011), 2011, Online unter URL: http://www.equipementsdelaroute.developpement-

durable.gouv.fr/modification-de-I-arrete-du-24-novembre-1967-suite-a379.html (Übersetzung durch Filippo Moraldi, Università degli Studi di Firenze) [Stand: 11.11.2015]

\section{[IISR 2013a]}

Instruction Interministérielle sur la signalisation routière du 22 octobre 1963 (IISR) - 4ème PARTIE: Signalisation de prescription, modifié par les arrêtés du 31 décembre 2012 (JO du 17 janvier 2013), 2013, Online unter URL: http://www.equipementsdelaroute.developpementdurable.gouv.fr/modification-de-l-arrete-du-24-novembre-1967-suite-a379.html (Übersetzung durch Filippo Moraldi, Università degli Studi di Firenze) [Stand: 11.11.2015]

\section{[IISR 2013b]}

Instruction Interministérielle sur la signalisation routière du 22 octobre 1963 (IISR) - 5ème PARTIE: Signalisation d'indication, des services et de repérage, modifié par les arrêtés du 21 mars 2013 (JO du 25 avril 2013), 2013, Online unter URL: http://www.equipementsdelaroute.developpementdurable.gouv.fr/modification-de-l-arrete-du-24-novembre-1967-suite-a379.html (Übersetzung durch Filippo Moraldi, Università degli Studi di Firenze) [Stand: 11.11.2015] 


\section{[RVV 1990]}

Reglement verkeersregels en verkeerstekens 1990 (RVV 1990), Geldend op 10-11-2015, 2015, Online unter URL: http://wetten.overheid.nl/BWBR0004825/geldigheidsdatum 10-11-2015 (Übersetzung durch Verfasser) [Stand: 10.11.2015]

\section{[StVO 1970]}

Straßenverkehrs-Ordnung (StVO) vom 17. November 1970 (BGBI. I S. 1565), 1970, Online unter URL:http://www.bgbl.de/xaver/bgbl/start.xav?start=\%2F\%2F*[\%40attr id\%3D\%27bgbl170108.pdf \%27]\# bgbl \%2F\%2F*[\%40attr id\%3D\%27bgbl170108.pdf\%27] 1447150747001 [Stand: 04.11.2015]

\section{[StVO 2013]}

Straßenverkehrs-Ordnung (StVO) vom 6. März 2013 (BGBI. I S. 367), die durch Artikel 2 der Verordnung vom 15. September 2015 (BGBI. I S. 1573) geändert worden ist, 2013, Online unter URL: http://www.gesetze-im-internet.de/stvo 2013/ [Stand: 04.11.2015]

\section{[StVO 2015]}

Bundesgesetz vom 6. Juli 1960, mit dem Vorschriften über die Straßenpolizei erlassen werden (Straßenverkehrsordnung 1960 - StVO. 1960), Fassung vom 08.10.2015, 2015. Online unter URL: http://www.ris.bka.gv.at/GeltendeFassung.wxe?Abfrage=Bundesnormen\&Gesetzesnummer=1001 1336 [Stand: 10.11.2015]

\section{[SVG 2015]}

Strassenverkehrsgesetz (SVG) vom 19. Dezember 1958 (Stand am 20. Mai 2015), 2015, Online unter URL: https://www.admin.ch/opc/de/classifiedcompilation/19580266/201505200000/741.01.pdf [Stand: 04.11.2015]

\section{[SVV 2015]}

Signalisationsverordnung (SSV) vom 5. September 1979 (Stand am 1. Juni 2015), 2015, Online unter URL: https://www.admin.ch/opc/de/classifiedcompilation/19790235/201506010000/741.21.pdf [Stand: 04.11.2015]

\section{[VwV-StVO 2015]}

Allgemeine Verwaltungsvorschrift zur Straßenverkehrs-Ordnung (VwV-StVO) vom 22. Oktober 1998 in der Fassung vom 22. September 2015, 2015, Online unter URL: http://www.verwaltungsvorschriften-im-internet.de/bsvwvbund 26012001 S3236420014.htm [Stand: 04.11.2015] 


\section{Anhang A Verkehrszeichen für Radwege in den untersuchten europäischen Staaten}

\section{Schweiz:}

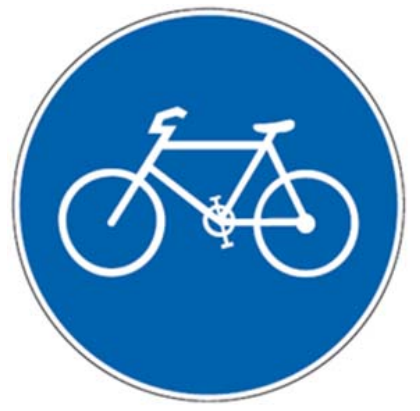

Signal 2.60

"Radweg“

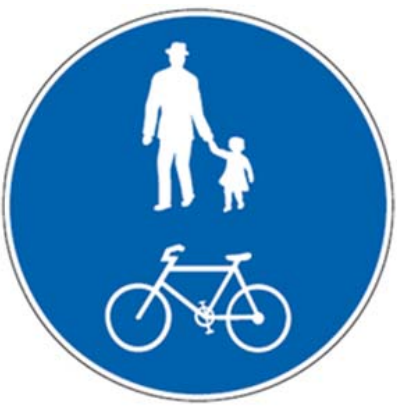

Signal 2.63.1, „gemeinsamer Rad- und Fussweg"

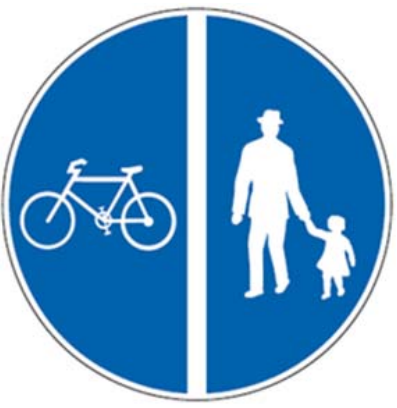

Signal 2.63 „Rad- und Fusswege mit getrennten Verkehrsflächen“"

Radwegebenutzungspflicht anordnende Verkehrszeichen in der Schweiz nach SVV [2015] (Bildquelle: SSV 2015)

\section{Österreich:}

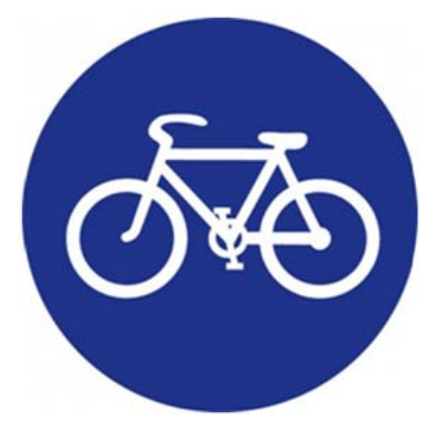

Z 16 „Radweg“

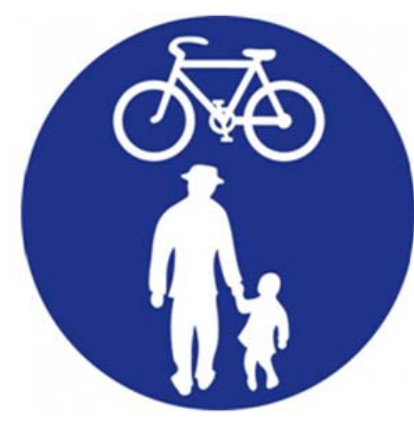

Z 17a.a "gemeinsamer Geh- und Radweg“"

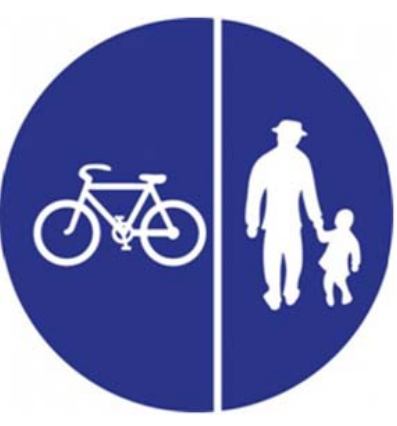

Z 17a.b "getrennter Geh- und Radweg“

Verkehrszeichen für „Radwege mit Benützungspflicht“ in Österreich nach § 52 StVO [2015] (Bildquelle: www.kommunalbedarf.at)

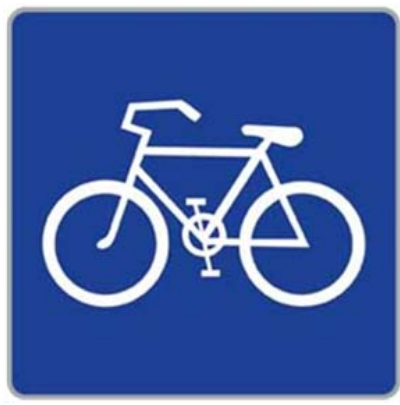

Z 27 „Radweg“

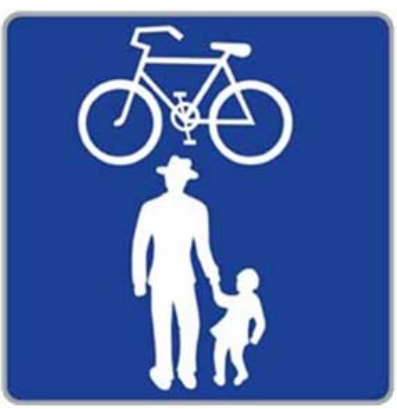

Z 28a "gemeinsamer Geh- und Radweg“

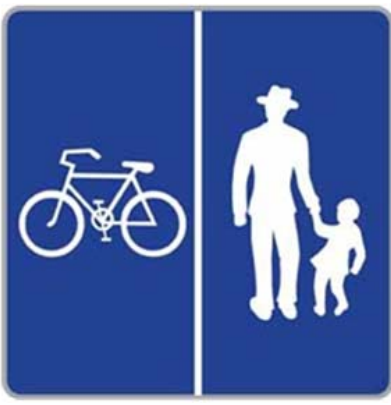

Z 28b „getrennter Geh- und Radweg“"

Verkehrszeichen für „Radwege ohne Benützungspflicht“ in Österreich nach § 53 StVo [2015] (Bildquelle: www.kommunalbedarf.at) 
Italien:

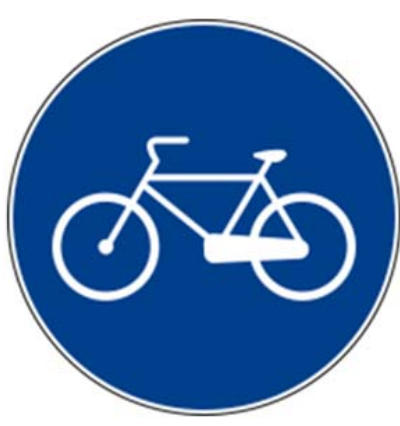

Figura II 90

"Radweg“

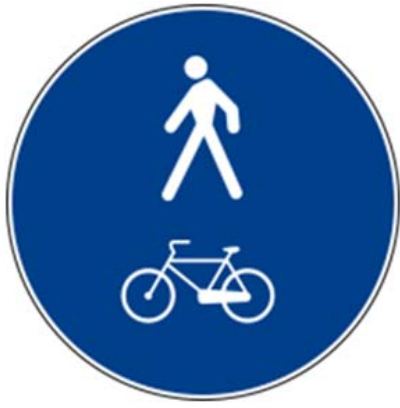

Figura II 92/b „gemeinsamer Gehund Radweg"

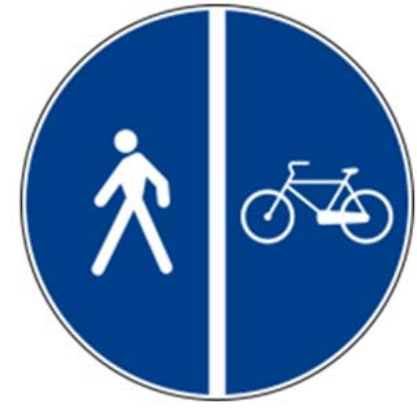

Figura II 90/a "getrennter Geh- und Radweg“"

Radwegebenutzungspflicht anordnende Verkehrszeichen in Italien nach Artikel 122, DPR 16.12.1992, $n^{\circ} 495$ [DPR 1992]

(Bildquelle: https://it.wikipedia.org)

\section{Niederlande:}

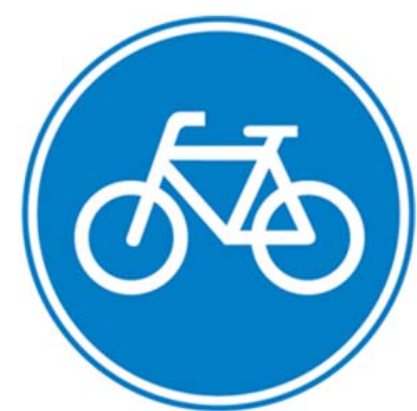

Zeichen G 11

„Radweg“

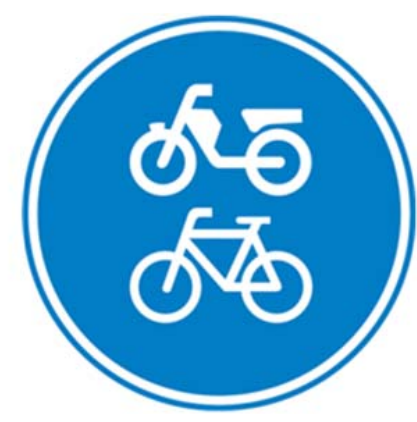

Zeichen G 12 a ,gemeinsamer Rad- und Mopedweg“"

Radwegebenutzungspflicht anordnende Verkehrszeichen in den Niederlanden nach Anlage 1, Kapitel G der RVV [2015] (Bildquelle: Verkeersborden en Verkeersregels in Nederland, Ministerie van Infrastructuur en Milieu, 2014)

\section{fietspad}

Kennzeichnung nicht benutzungspflichtiger Radwege in den Niederlanden mit Zeichen $\mathbf{G} 13$ gemäß Anlage 1, Kapitel G der RVV [2015] (Bildquelle: Verkeersborden en Verkeersregels in Nederland, Ministerie van Infrastructuur en Milieu, 2014) 
Frankreich:

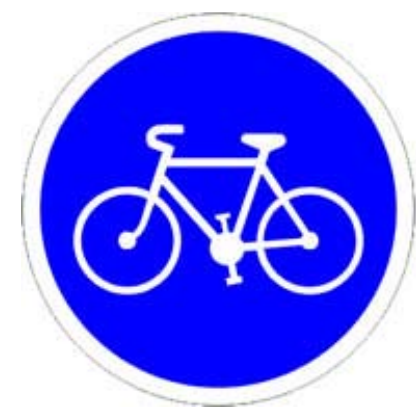

Radwegebenutzungspflicht anordnende Verkehrszeichen in Frankreich, Zeichen B 22a „Radweg“ nach IISR [2013a] (Bildquelle: www.saser.fr)

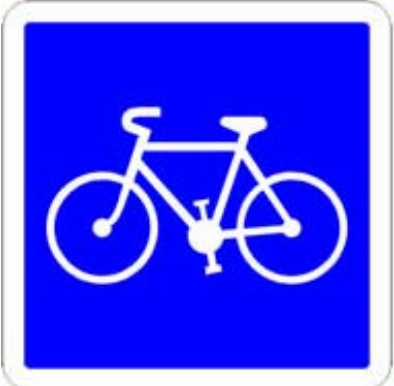

Kennzeichnung nicht benutzungspflichtiger Radwege in Frankreich, Zeichen C 113 „empfohlener Radweg“ nach IISR [2013b] (Bildquelle: www.saser.fr) 


\section{Anhang B Empfehlungen zur Radwegebenutzungspflicht nach Main- zer Leitfaden}

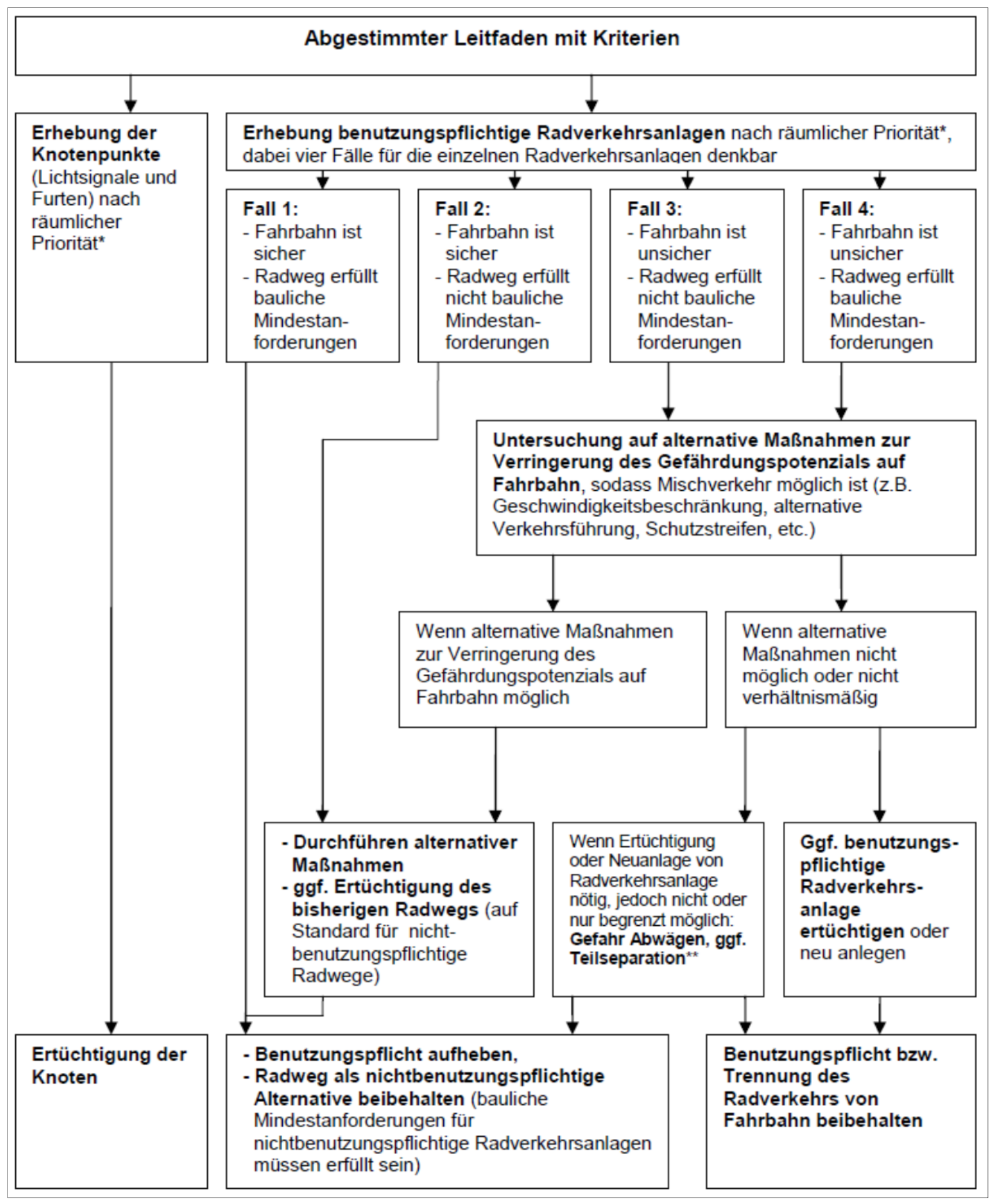

[Quelle: Klöpfer et al. 2011] 


\section{Anhang C Unfallkenngrößen ohne Projektion der Radverkehrsstärken}

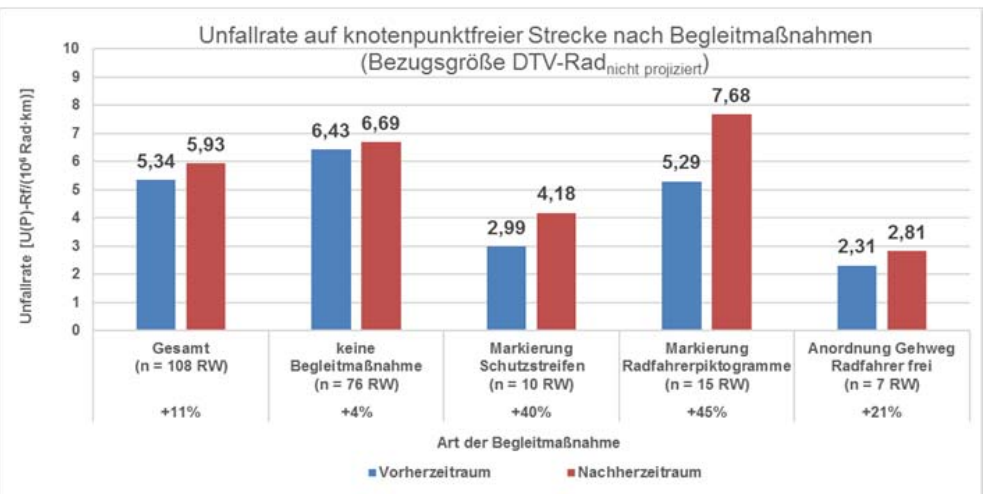

Unfallraten auf knotenpunktfreier Strecke (Bezug DTV-Rad nicht projiziert)

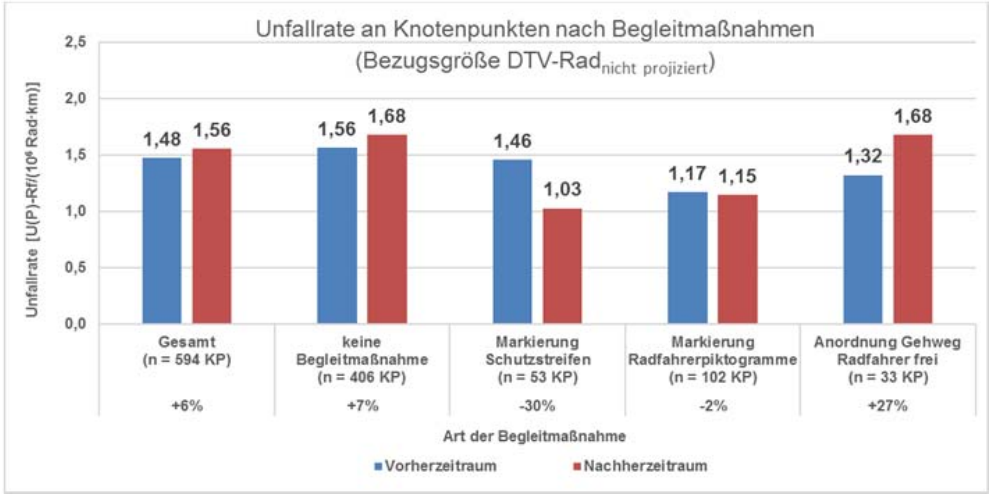

Unfallraten an Knotenpunkten (Bezug DTV-Rad ${ }_{\text {nicht projiziert) }}$

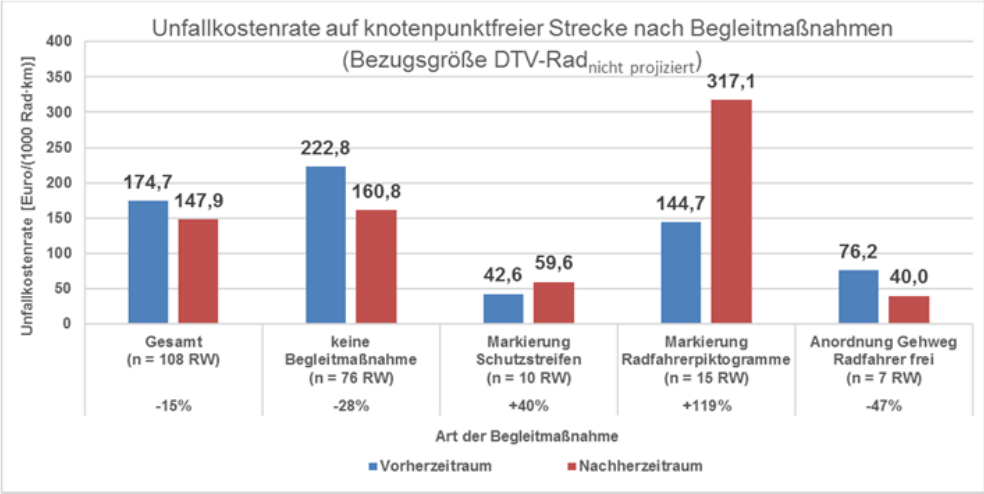

Unfallkostenraten auf knotenpunktfreier Strecke (Bezug DTV-Rad ${ }_{\text {nicht projiziert) }}$

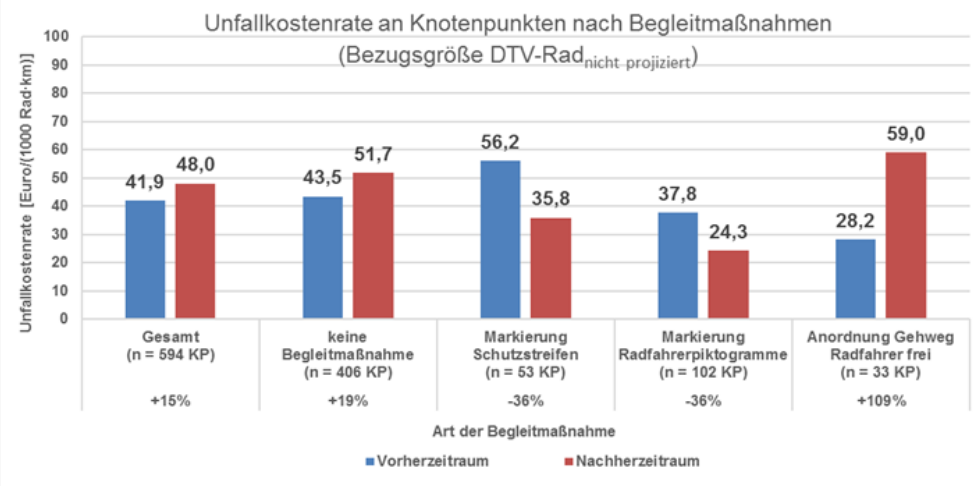

Unfallkostenraten an Knotenpunkten (Bezug DTV-Rad ${ }_{\text {nicht projiziert) }}$ 


\section{Anhang D Unfallkenngrößen mit Projektion der Radverkehrsstärken}

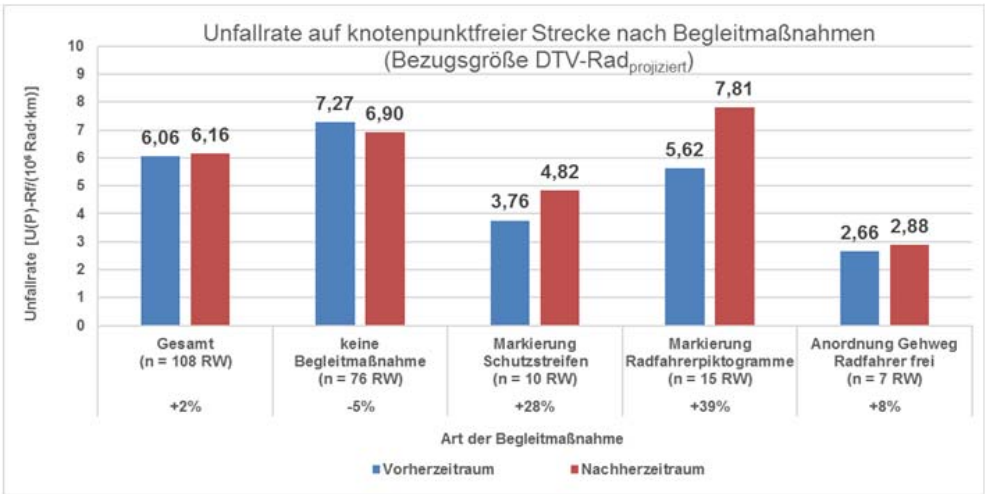

Unfallraten auf knotenpunktfreier Strecke (Bezug DTV-Rad projiziert)

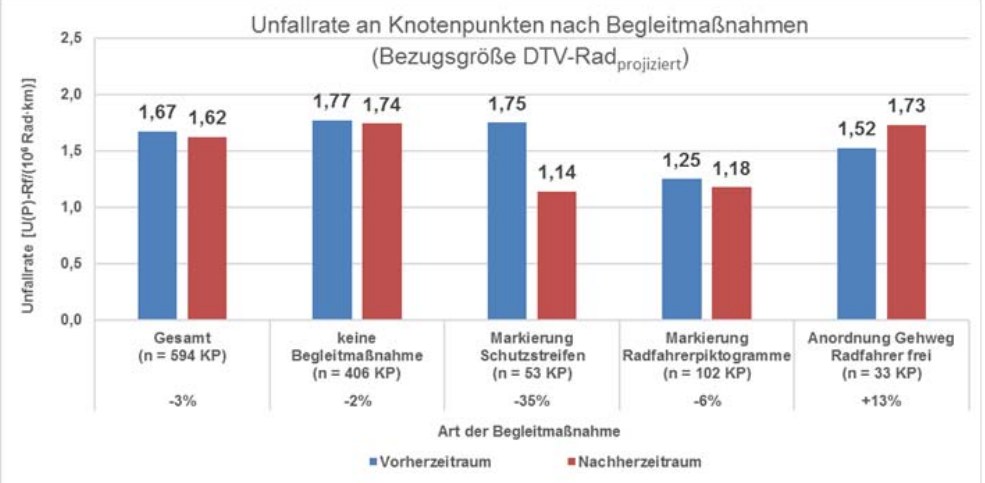

Unfallraten an Knotenpunkten (Bezug DTV-Rad projiziert)

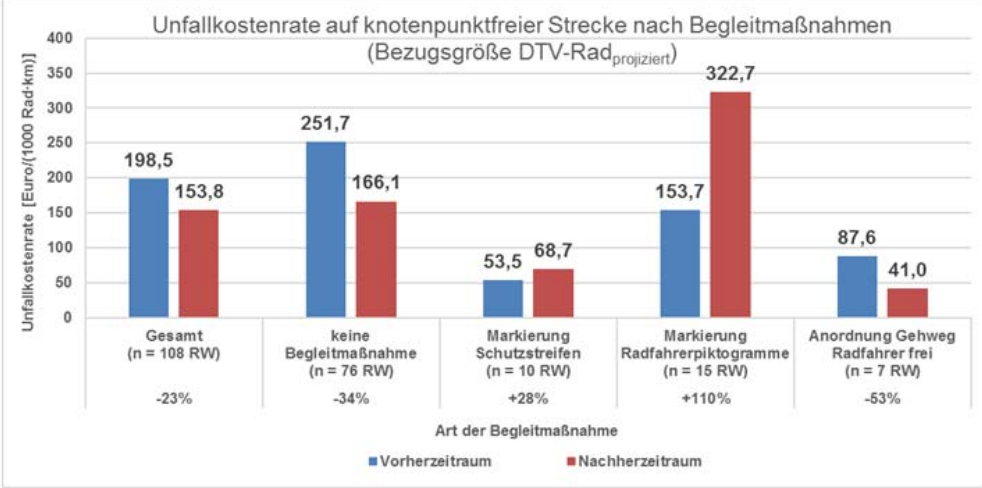

Unfallkostenraten auf knotenpunktfreier Strecke (Bezug DTV-Rad ${ }_{\text {projiziert) }}$

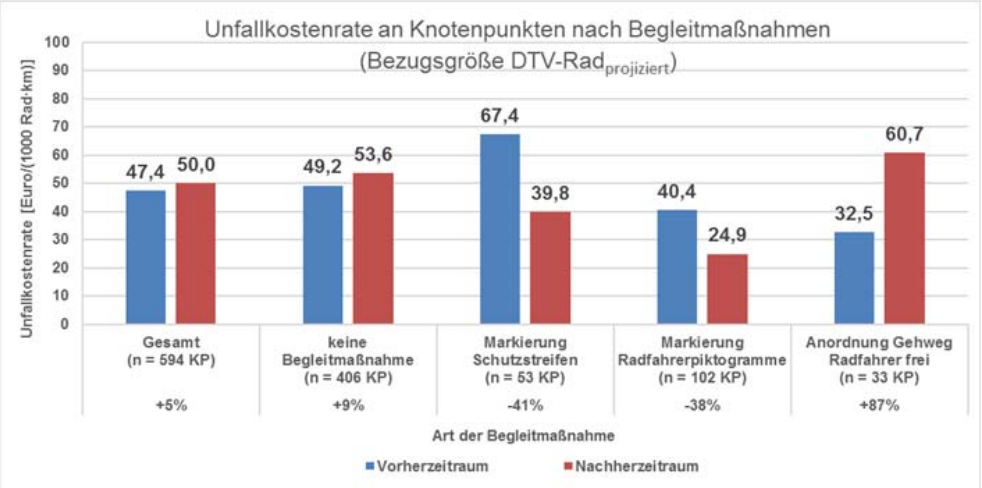

Unfallkostenraten an Knotenpunkten (Bezug DTV-Rad ${ }_{\text {projiziert) }}$ 


\section{Anhang E Unfallrate und Unfallkostenrate nach Kraftfahrzeugver- kehrsstärken an Radwegen mit aufgehobener Benutzungs- pflicht ohne Begleitmaßnahmen}

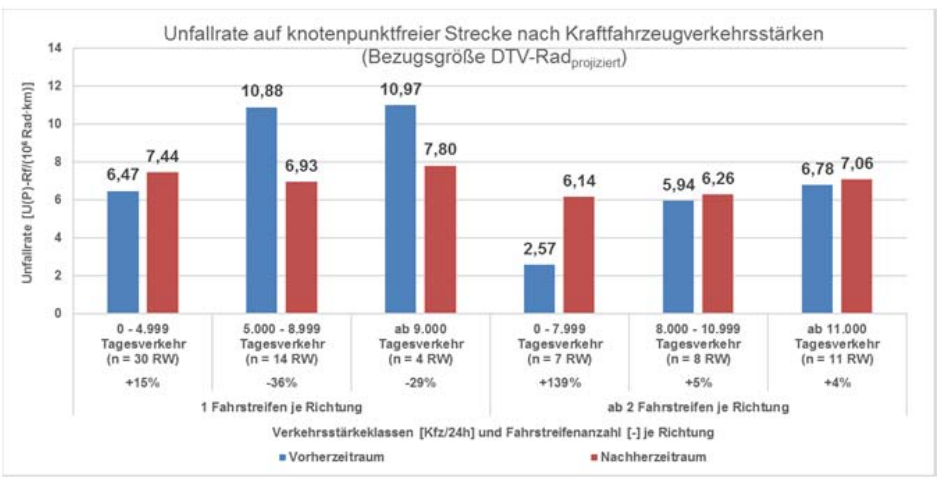

Unfallraten auf knotenpunktfreier Strecke (Bezug DTV-Rad projiziert )

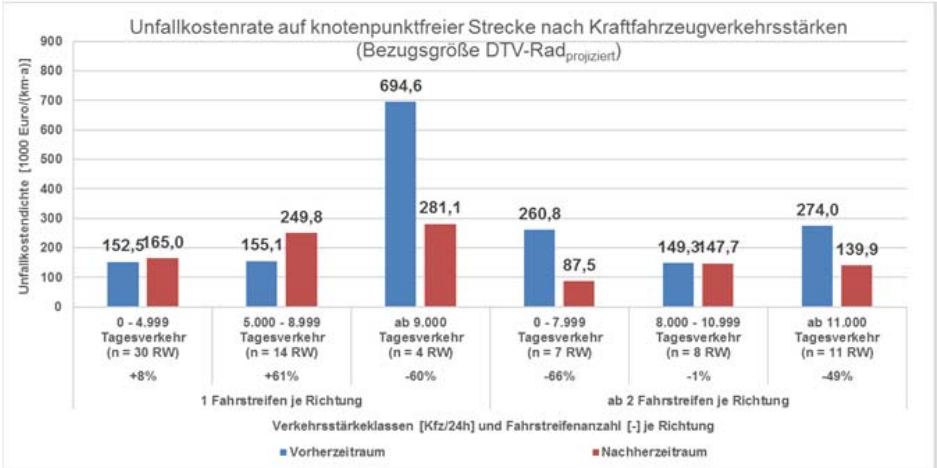

Unfallkostenraten auf knotenpunktfreier Strecke (Bezug DTV-Rad projiziert $_{\text {) }}$

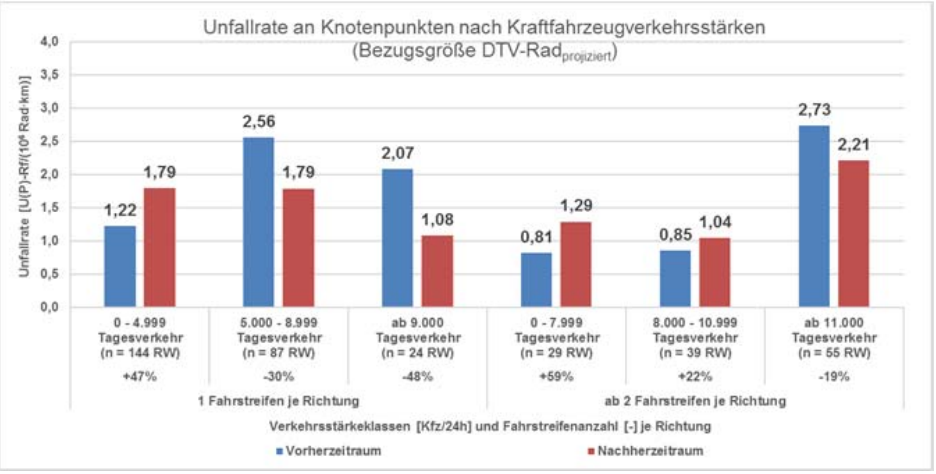

Unfallraten an Knotenpunkten (Bezug DTV-Rad projiziert)

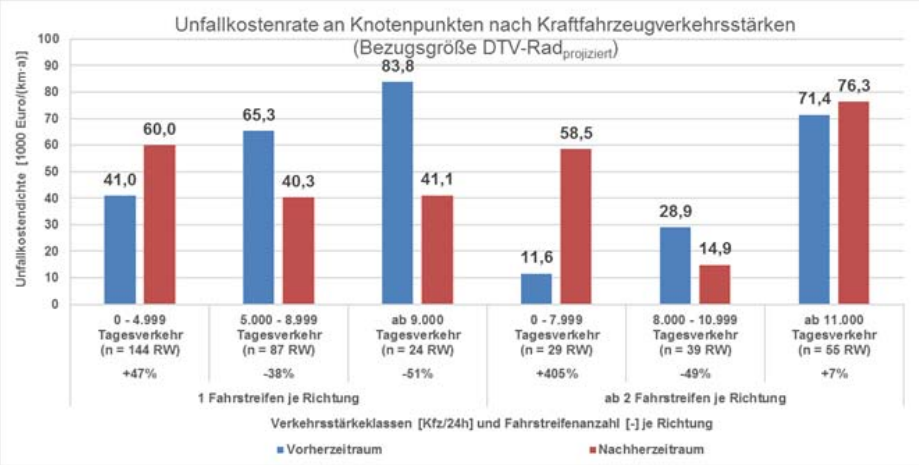

Unfallkostenraten an Knotenpunkten (Bezug DTV-Rad projiziert $_{\text {) }}$ 


\section{Anhang F Unfallrate und Unfallkostenrate nach Radverkehrsstärken an Radwegen mit aufgehobener Benutzungspflicht ohne Begleitmaßnahmen}
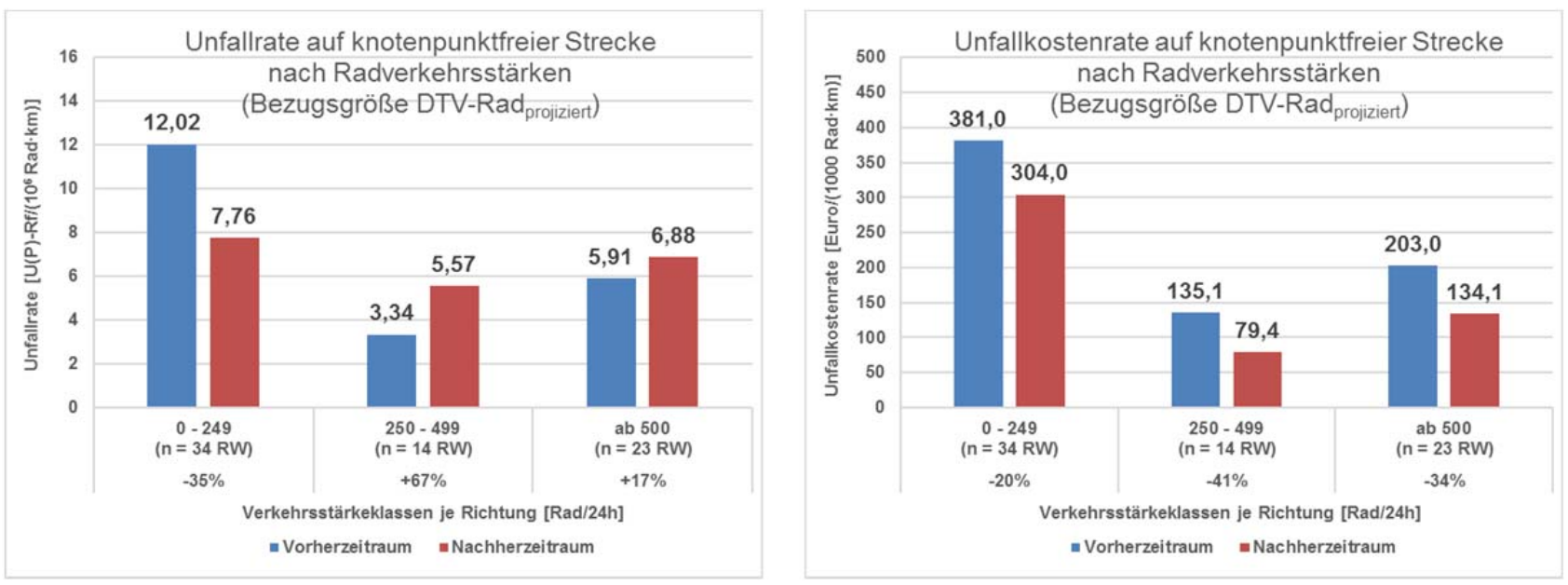

Unfallraten und Unfallkostenraten auf knotenpunktfreier Strecke (Bezug DTV-Rad projiziert) $_{\text {) }}$
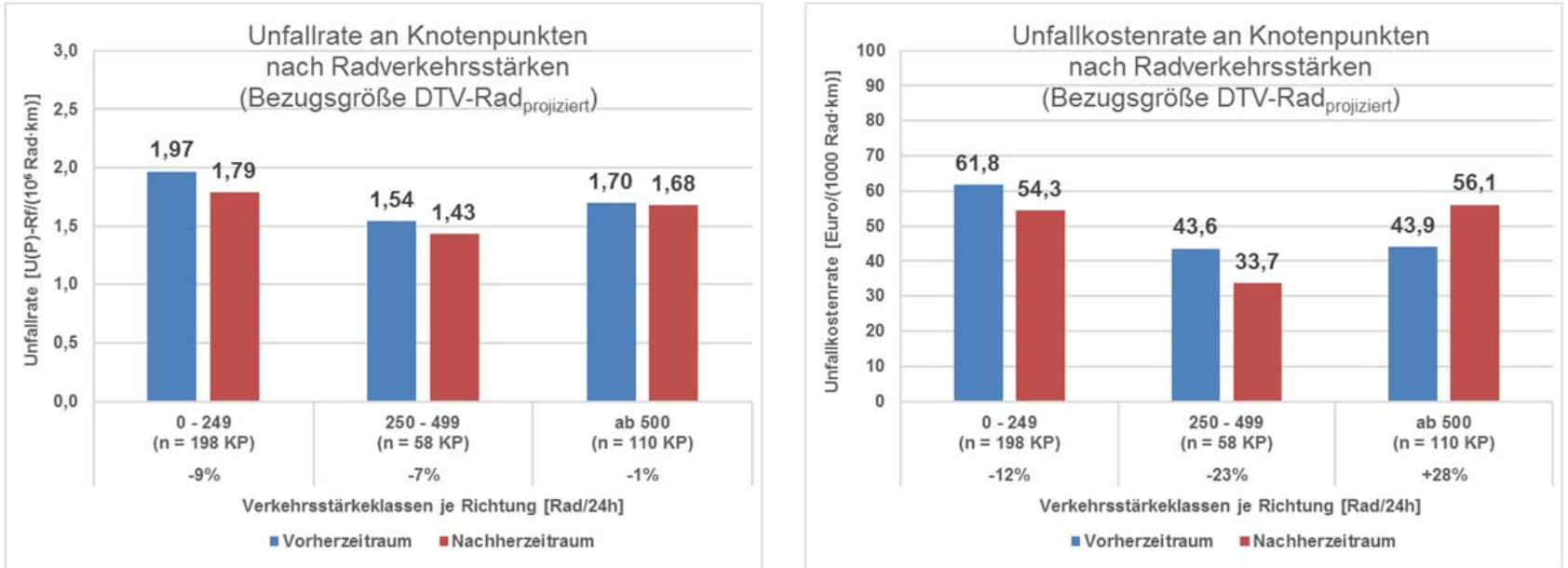

Unfallraten und Unfallkostenraten an Knotenpunkten (Bezug DTV-Rad projiziert) 


\section{Anhang G Unfallrate und Unfallkostenrate nach Fahrstreifenanzahl an Radwegen mit aufgehobener Benutzungspflicht ohne Begleitmaßnahmen}
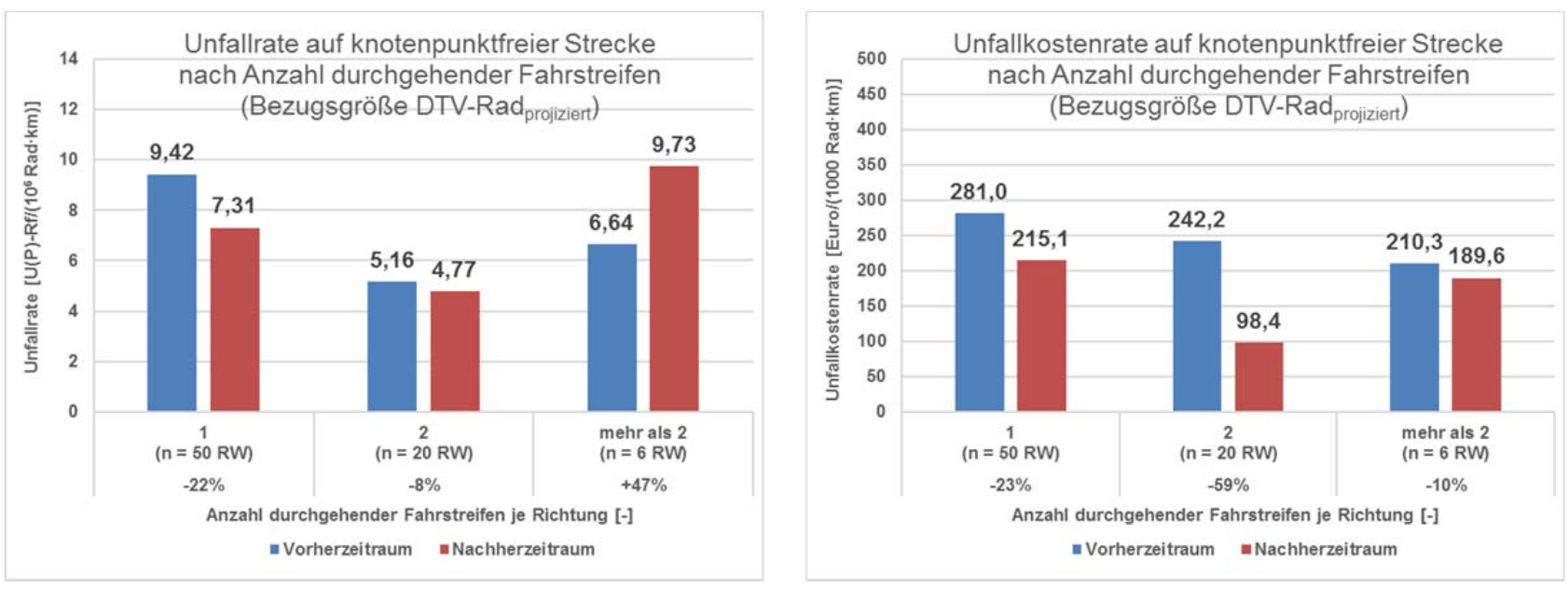

Unfallraten und Unfallkostenraten auf knotenpunktfreier Strecke (Bezug DTV-Rad ${ }_{\text {projiziert) }}$
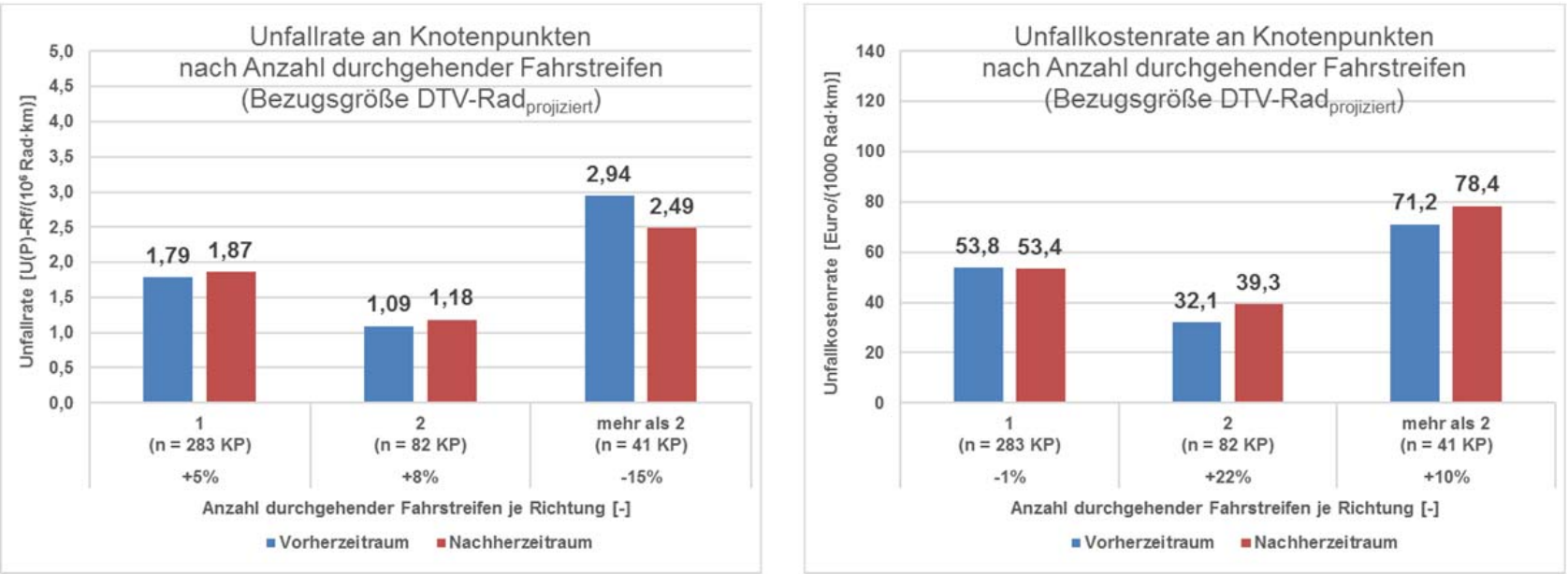

Unfallraten und Unfallkostenraten an Knotenpunkten (Bezug DTV-Rad ${ }_{\text {projiziert }}$ ) 


\section{Anhang H Unfallrate und Unfallkostenrate nach Breite des rechten Fahrstreifens an Radwegen mit aufgehobener Benutzungs- pflicht ohne Begleitmaßnahmen}
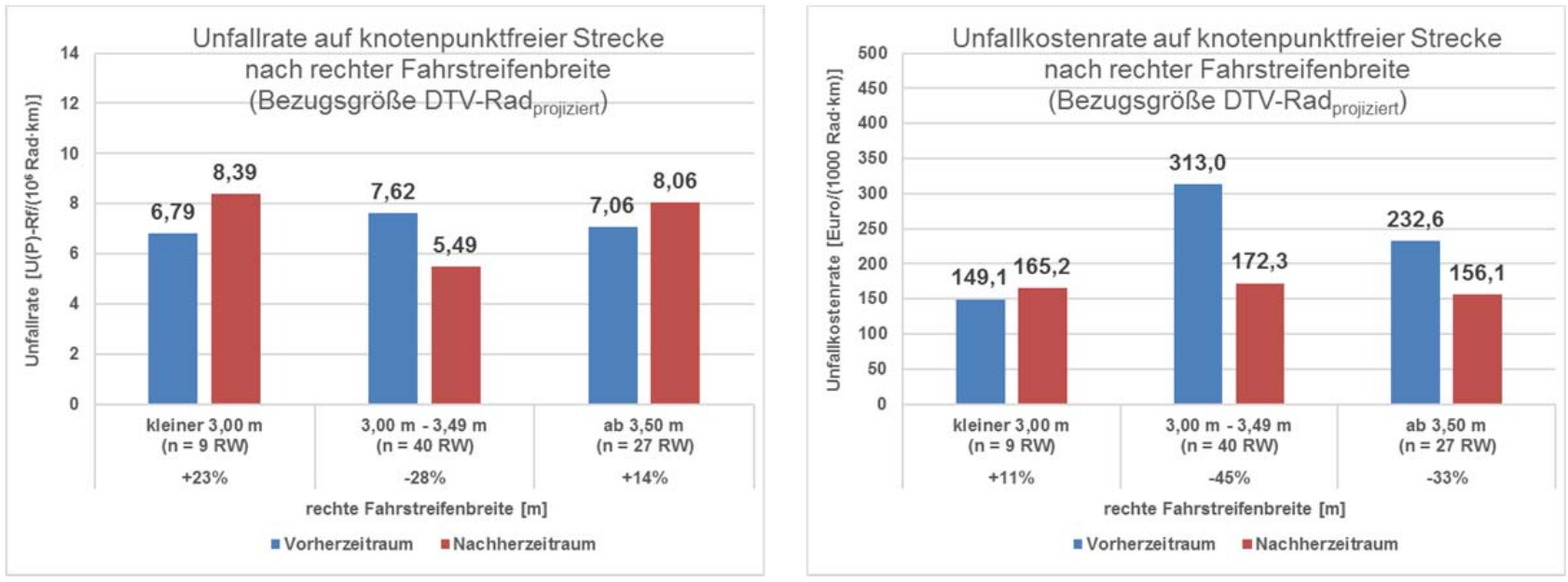

Unfallraten und Unfallkostenraten auf knotenpunktfreier Strecke (Bezug DTV-Rad ${ }_{\text {projiziert }}$ )
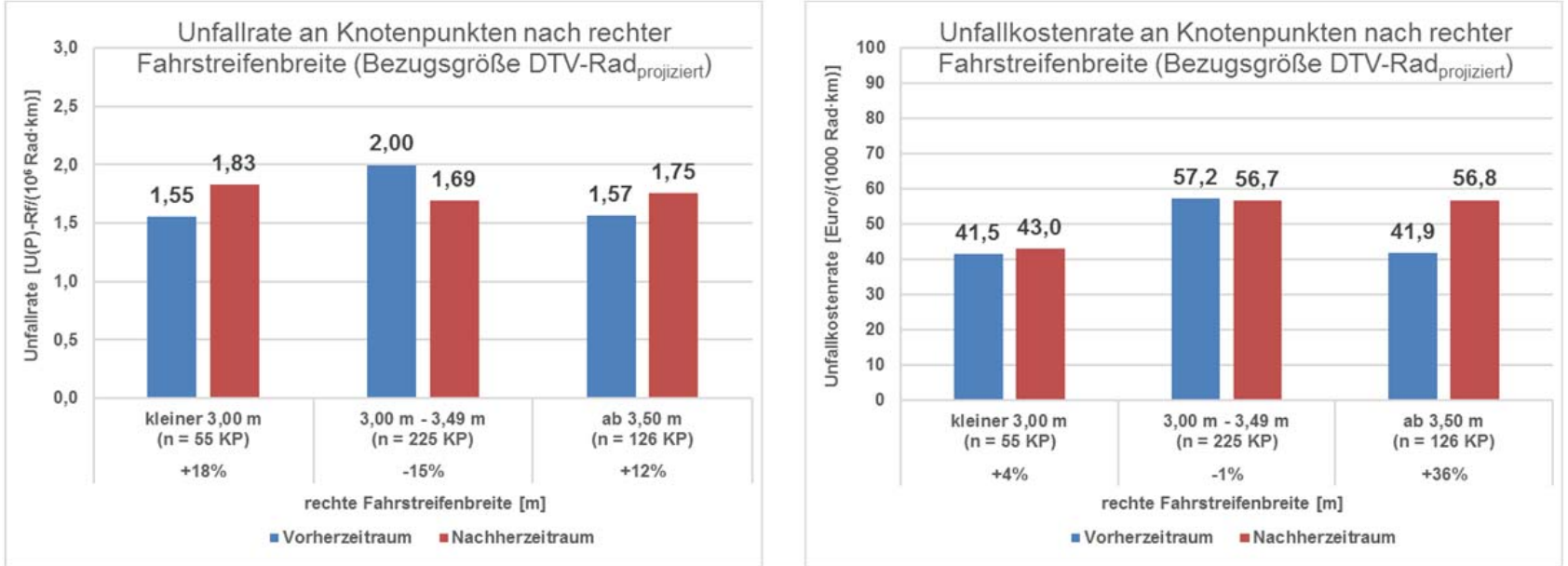

Unfallraten und Unfallkostenraten an Knotenpunkten (Bezug DTV-Rad projiziert) 


\section{Anhang I Radunfallgeschehen nach Begleitmaßnahmen}

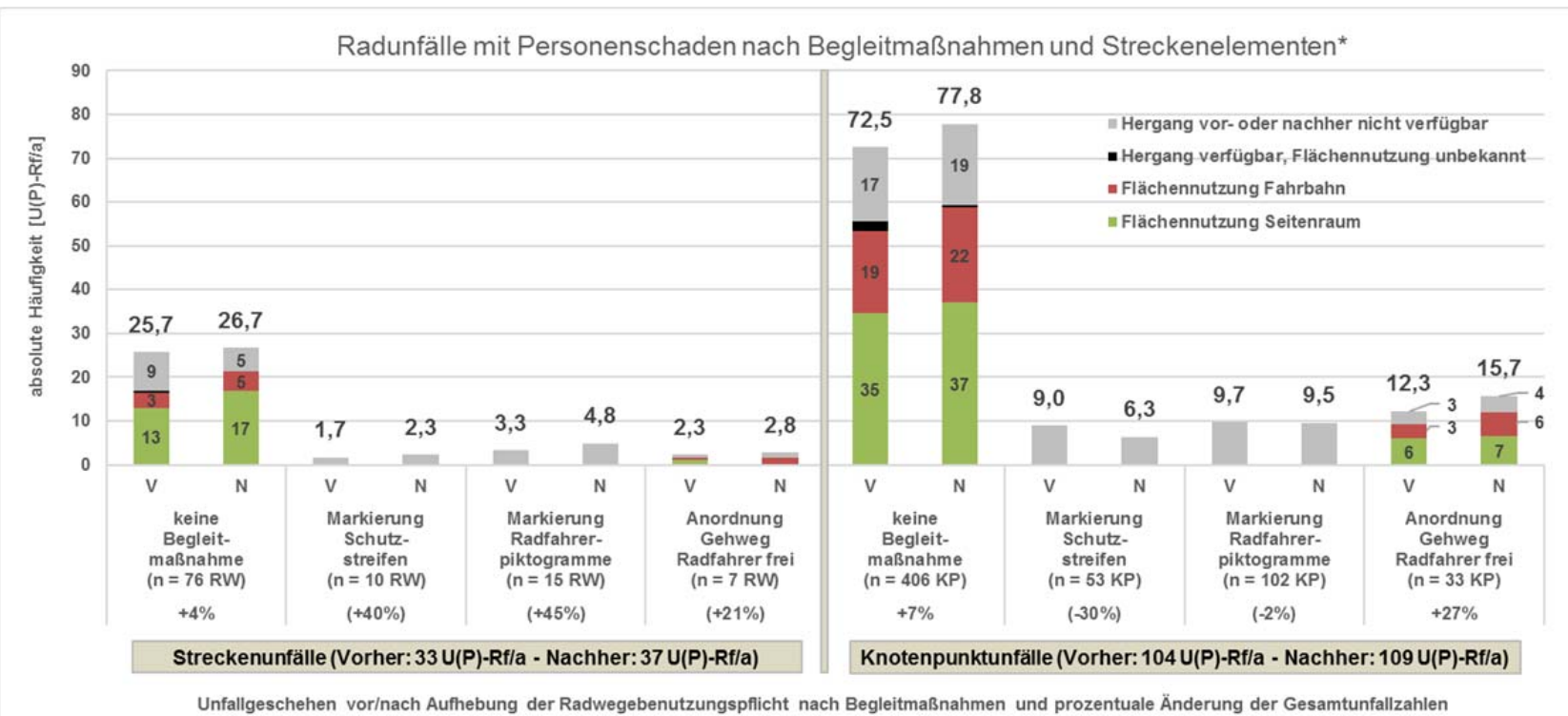

*ohne Berücksichtigung der Radverkehrsentwicklung

\section{Verteilung des Radunfallgeschehens nach Begleitmaßnahmen an den untersuchten 108 Streckenzügen und 594 Knotenpunkten}

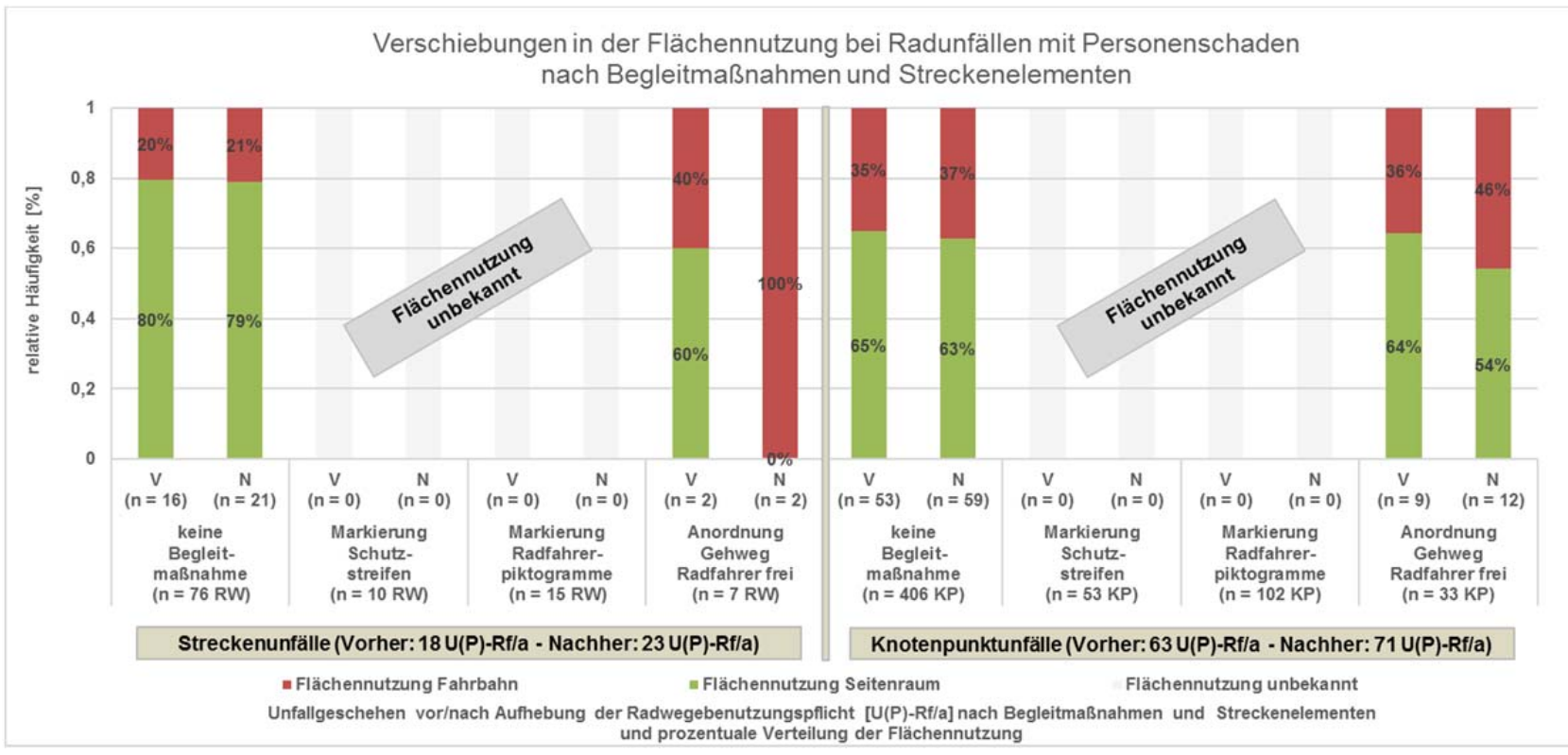

Verschiebungen in der Flächennutzung bei Radunfällen nach Begleitmaßnahmen an den untersuchten 108 Streckenzügen und 594 Knotenpunkten 


\section{Anhang J Radunfallgeschehen nach Unfallkategorien}

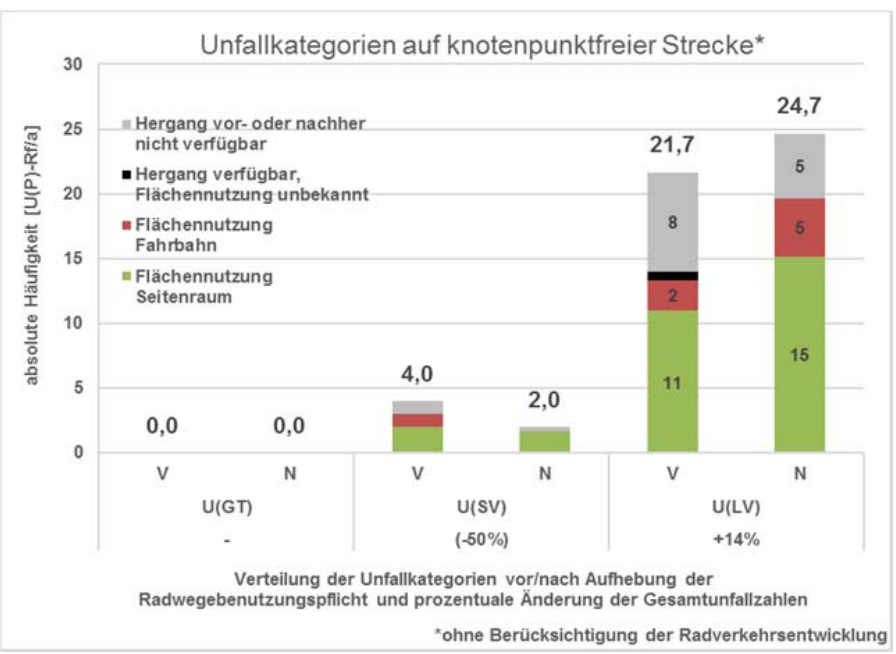

Verteilung der Unfallkategorien bei Radunfällen auf knotenpunktfreier Strecke ( $n=76$ Streckenzüge ohne Begleitmaßnahme)

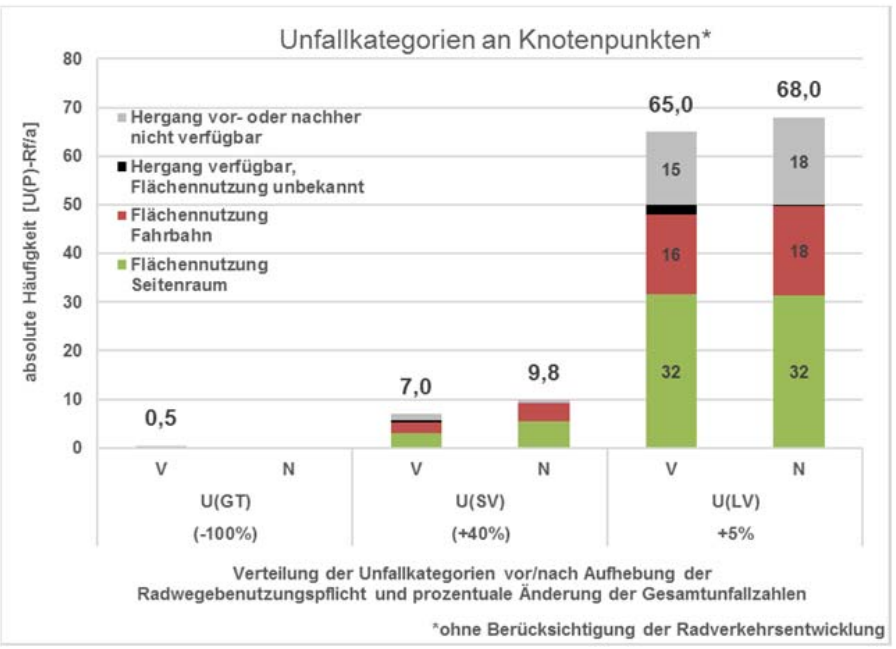

Verteilung der Unfallkategorien bei Radunfällen an Knotenpunkten ( $n=406$ Knotenpunkte ohne Begleitmaßnahmen)

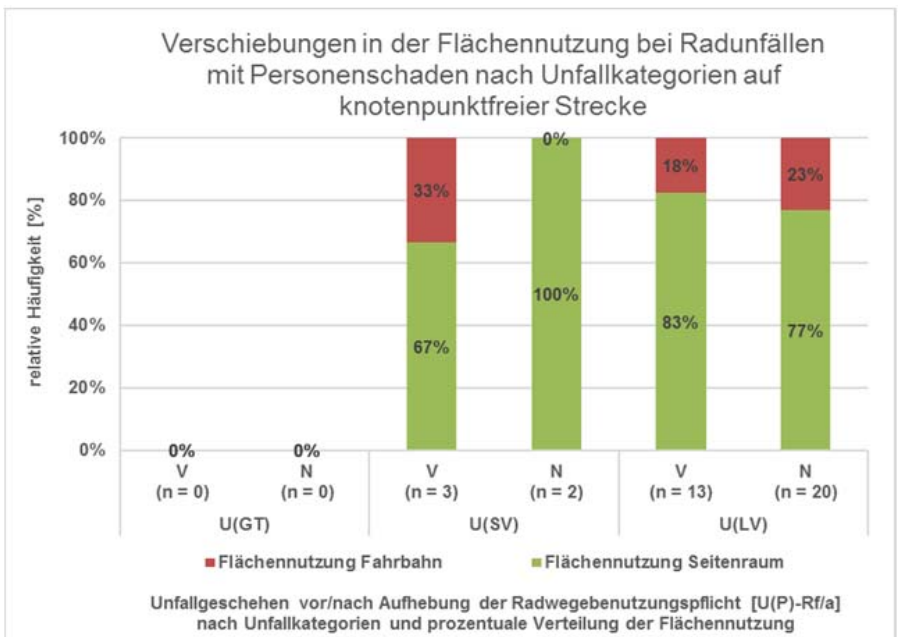

Verschiebungen in der Flächennutzung bei Radunfällen auf knotenpunktfreier Strecke ( $n=76$ Streckenzüge ohne Begleitmaßnahme) 


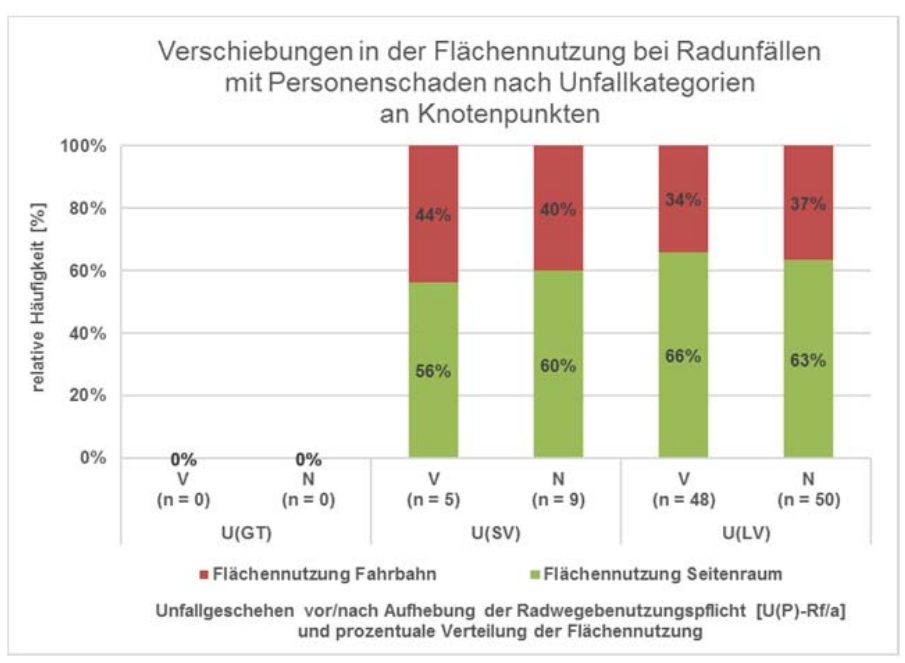

Verschiebungen in der Flächennutzung bei Radunfällen an Knotenpunkten ( $n=406$ Knotenpunkte ohne Begleitmaßnahmen) 


\section{Anhang K Radunfallgeschehen nach Unfalltypen}

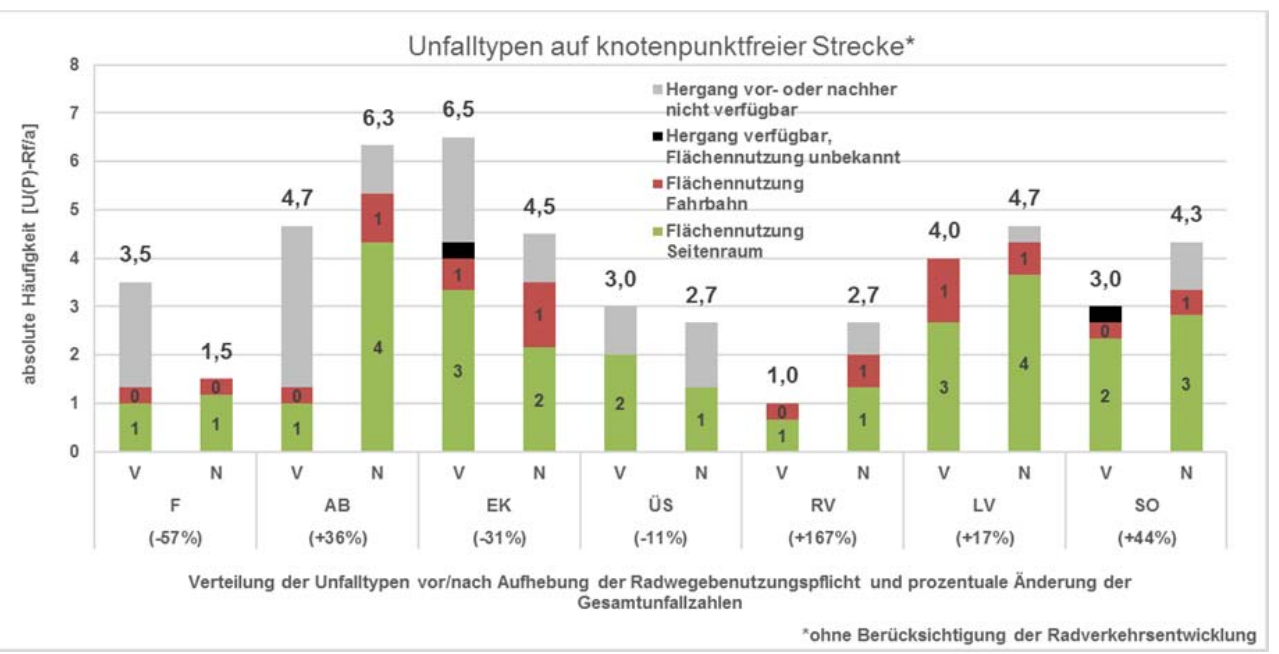

Verteilung der Unfalltypen bei Radunfällen

auf knotenpunktfreier Strecke ( $n=76$ Streckenzüge ohne Begleitmaßnahme)

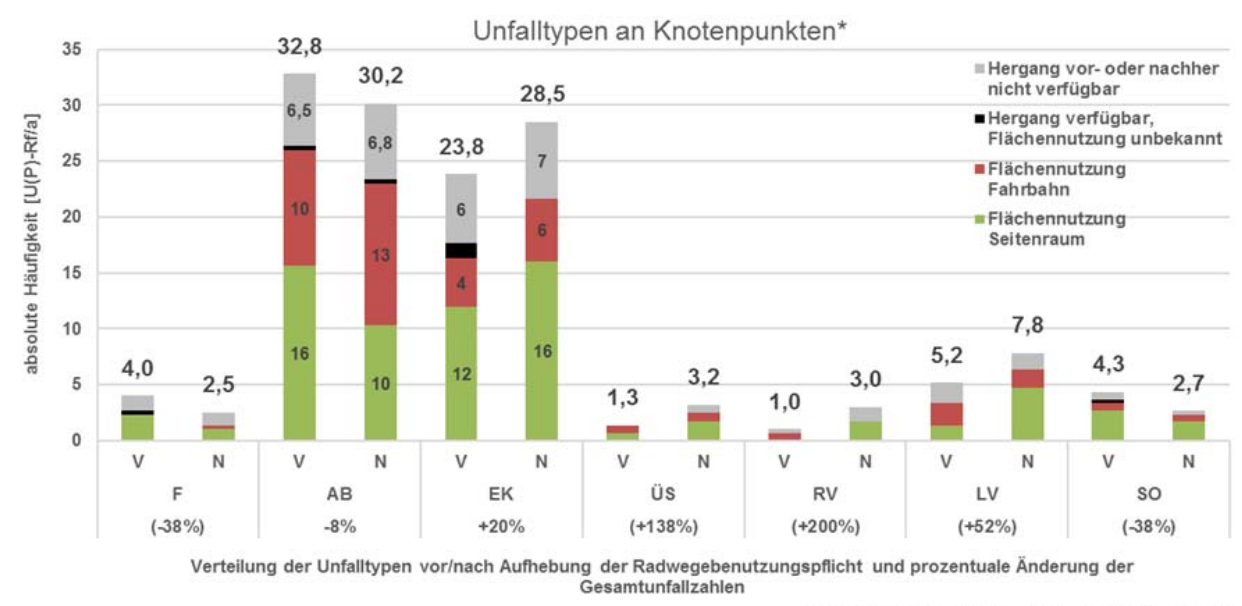

*ohne Berücksichtigung der Radverkehrsentwicklung

Verteilung der Unfalltypen bei Radunfällen an Knotenpunkten ( $n=406$ Knotenpunkte ohne Begleitmaßnahmen)

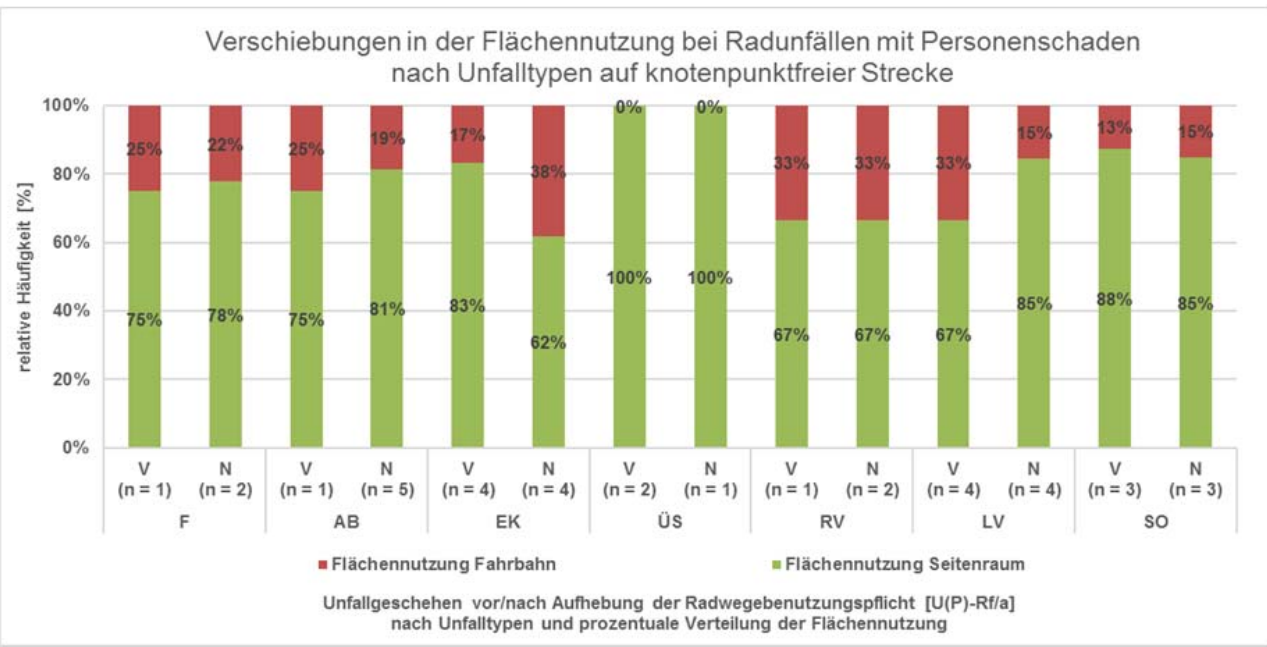

Verschiebungen in der Flächennutzung bei Radunfällen auf knotenpunktfreier Strecke ( $n=76$ Streckenzüge ohne Begleitmaßnahme) 


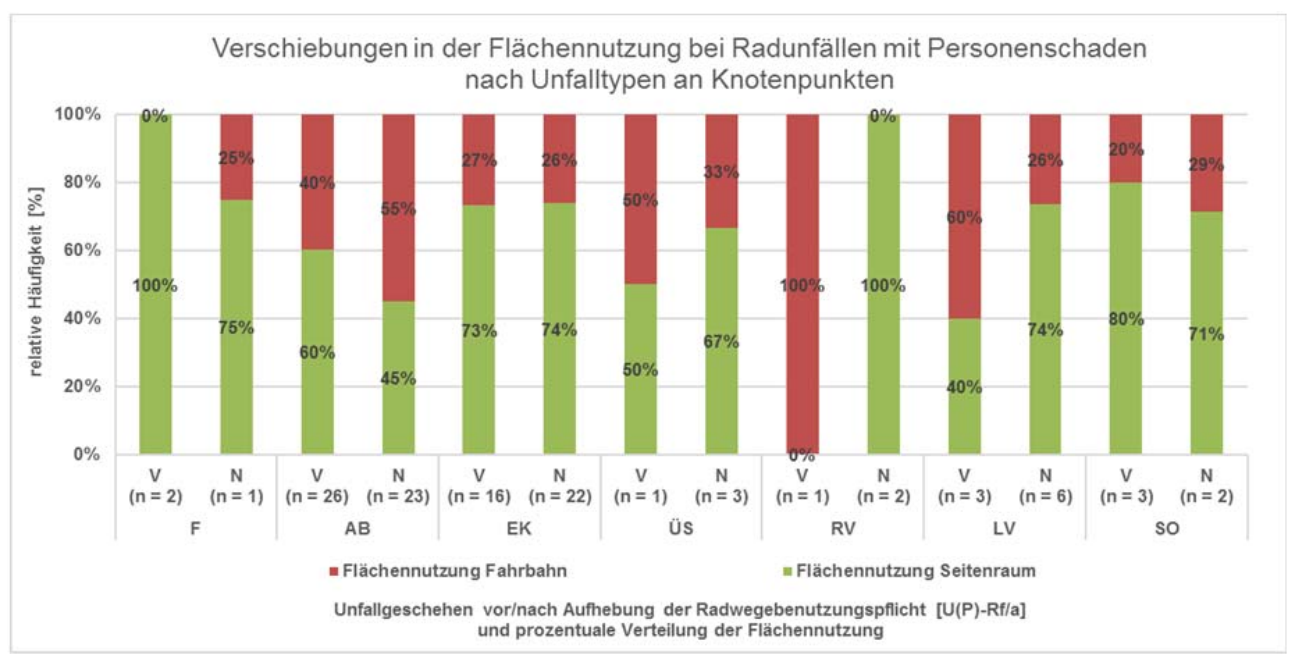

Verschiebungen in der Flächennutzung bei Radunfällen an Knotenpunkten ( $n=406$ Knotenpunkte ohne Begleitmaßnahmen) 


\section{Anhang $L \quad$ Radunfallgeschehen nach Hauptunfallverursacher}

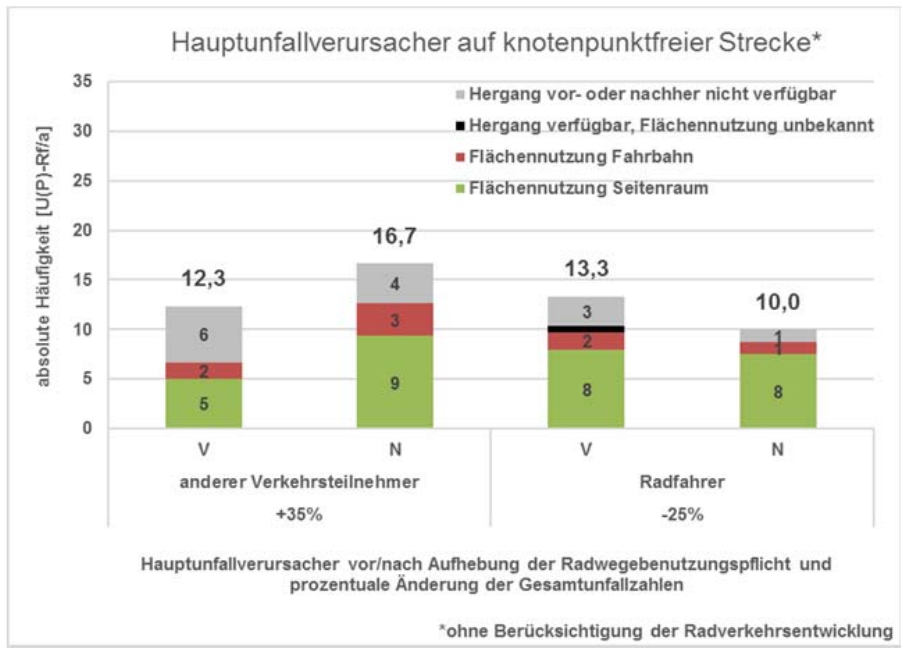

Hauptunfallverursacher von Radunfällen auf knotenpunktfreier Strecke ( $n=76$ Streckenzüge ohne Begleitmaßnahme)

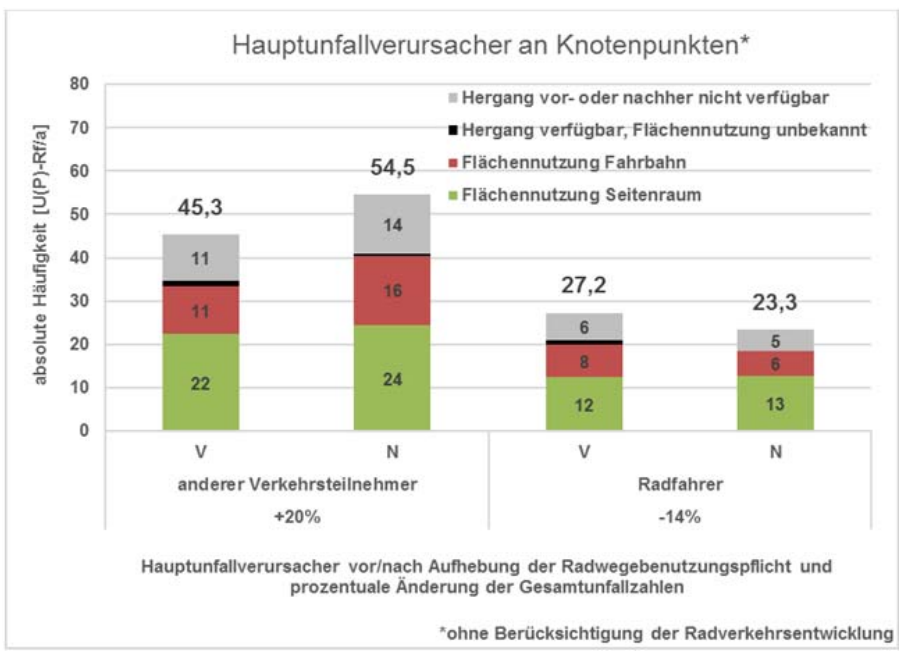

Hauptunfallverursacher von Radunfällen an Knotenpunkten ( $n=406$ Knotenpunkte ohne Begleitmaßnahmen)

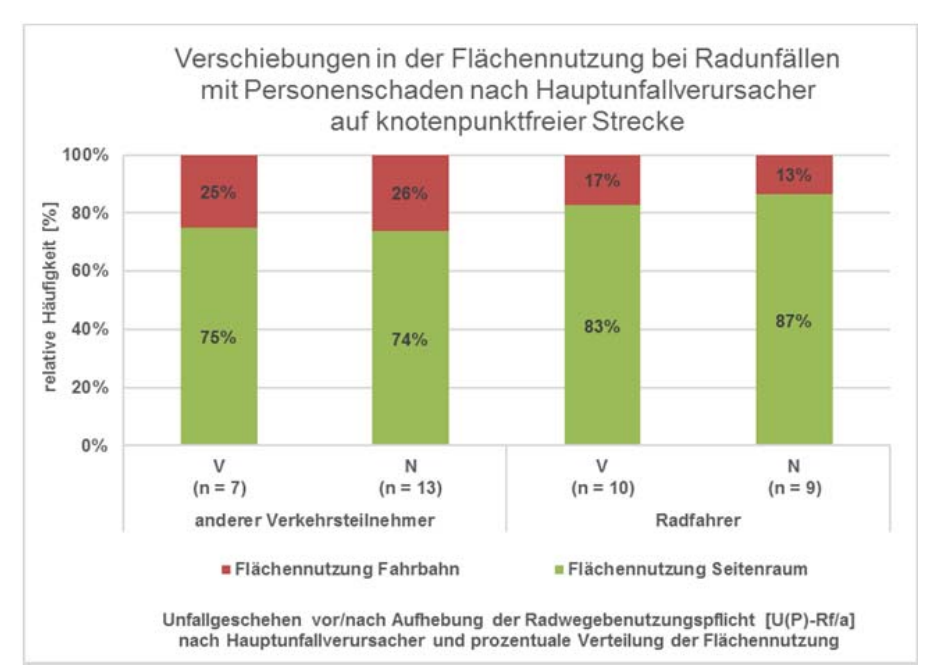

Verschiebungen in der Flächennutzung bei Radunfällen auf knotenpunktfreier Strecke ( $n=76$ Streckenzüge ohne Begleitmaßnahme) 


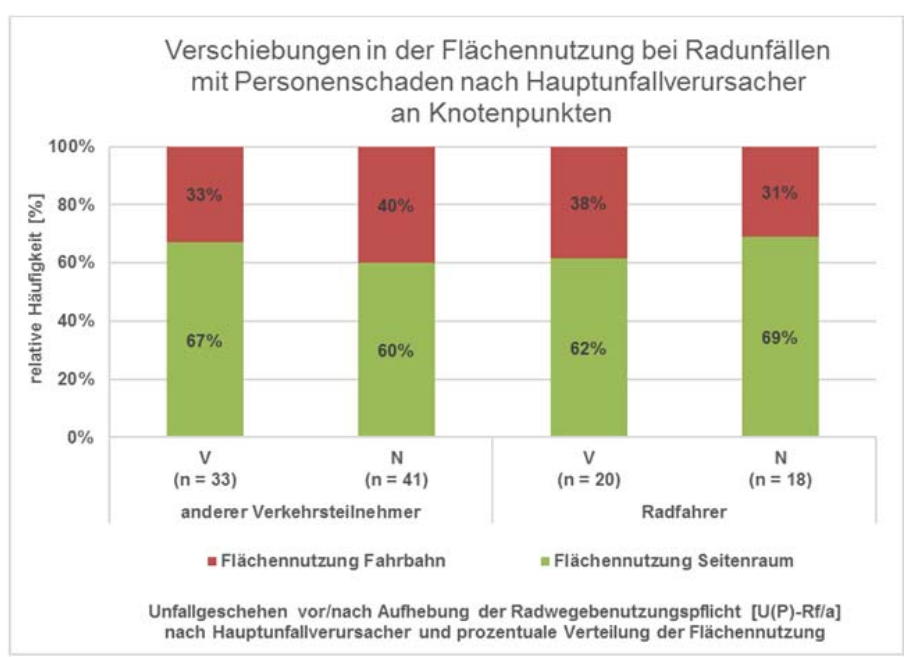

Verschiebungen in der Flächennutzung bei Radunfällen an Knotenpunkten ( $n=406$ Knotenpunkte ohne Begleitmaßnahmen) 


\section{GDV \\ DIE DEUTSCHEN VERSICHERER}

Gesamtverband der Deutschen Versicherungswirtschaft e. V.

Wilhelmstraße 43 / 43G, 10117 Berlin

Postfach 0802 64, 10002 Berlin

Telefon 030 / 2020 - 50 00, Fax 030 / 2020 - 6000

Internet: www.gdv.de, www.udv.de 